\title{
A Spatial Discretization Scheme for Solving the Transport Equation on Unstructured Grids of Polyhedra
}

\section{Los Alamos}


This thesis was accepted by the Department of Nuclear Engineering, Texas AEM University, College Station, Texas, in partial fulfillment of the requirements for the degree of Doctor of Philosophy. The text and illustrations are the independent work of the author and only the front matter has been edited by the IM-1 Writing and Editing Staff to conform with Department of Energy and Los Alamos National Laboratory publication policies.

An Affirmative Action/Equal Opportunity Employer

This report was prepared as an account of work sponsored by an agency of the United States Government. Neither The Regents of the University of California, the United States Government nor any agency thereof, nor any of their employees, makes any warranty, express or implied, or assumes any legal liability or responsibility for the accuracy, completeness, or usefulness of any information, apparatus, product, or process disclosed, or represents that its use would not infringe privately owned rights. Reference herein to any specific commercial product, process, or service by trade name, trademark, manufacturer, or otherwise, does not necessarily constitute or imply its endorsement, recommendation, or favoring by The Regents of the University of California, the United States Government, or any agency thereof. The views and opinions of authors expressed herein do not necessarily state or reflect those of The Regents of the University of California, the United States Government, or any agency thereof. Los Alamos National Laboratory strongly supports academic freedom and a researcher's right to publish; as an institution, however, the Laboratory does not endorse the viewpoint of a publication or guarantee its technical correctness. 


\author{
A Spatial Discretization Scheme \\ for Solving the Transport Equation on \\ Unstructured Grids of Polyhedra \\ Kelly Glen Thompson
}


To my loving family, Lori, Allison and Jessica. 


\section{ACKNOWLEDGEMENTS}

This research is the culmination of the efforts of many individuals to whom I owe my most sincere appreciation. My greatest thanks I reserve for my advisor, Marvin Adams. Without his tireless ability to teach and motivate, this work would not have been completed. I would further like to thank Drs. Dmitriy Yu. Anistratov, Raytcho Lazarov and Paul Nelson, Jr. for serving on my committee and for providing me with additional perspectives.

I would like to thank Mike Zika and Paul Nowak at Lawrence Livermore National Laboratory for insight and encouragement during this work. Thanks goes also to the Shavano team at Los Alamos National Laboratory for providing me with many tools to support my work. Additional thanks go to Jon Dahl for tirelessly answering questions about PARTISN; to Steve Nolen for his encouragement and help with using the ASCI machines at LANL; and to Dave Court for his help in extracting problem specifics for the ATR MOX simulation from MCNP input files.

I owe thanks to the National Science Foundation, the Oak Ridge Institute for Science and Education, Los Alamos National Laboratory and Lawrence Livermore National Laboratory for providing financial support. Without this assistance this work would not have been possible.

I would like to thank the transport research team at Texas A\&M University for providing encouragement, helpful discussions and for adding a little humor to my life. This team consists of Chris Castrianni, Mike Zika, Shawn Pautz, Daryl Hawkins, Jae Chang and Chris Gesh. A special thanks goes to Hiromi Kato for providing the SMaRT input decks used in modeling the ATR MOX problem. 


\section{TABLE OF CONTENTS}

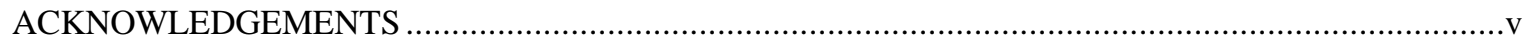

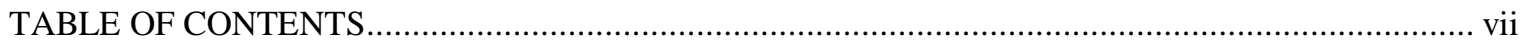

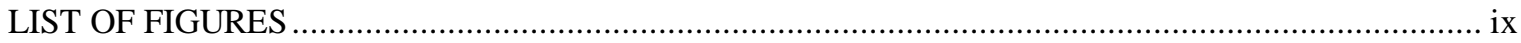

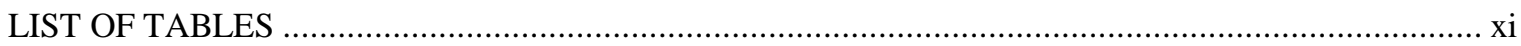

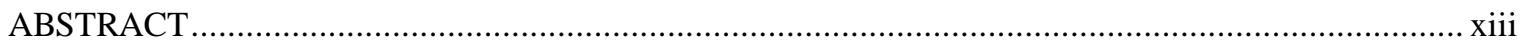

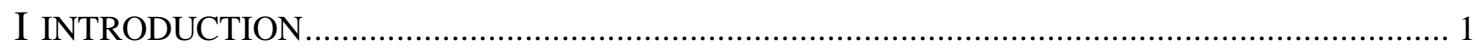

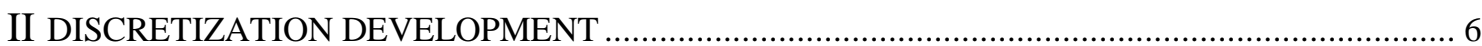

II. A. Goals for the Discretization ...............................................................................

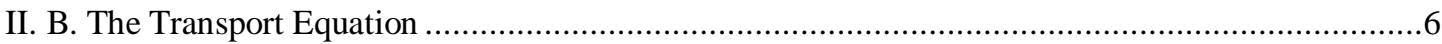

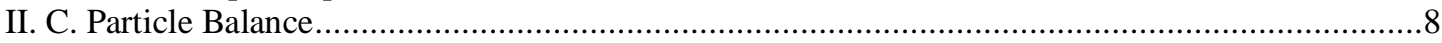

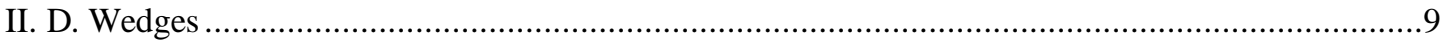

II. E. 1. Closure Development - Introduction ....................................................................... 10

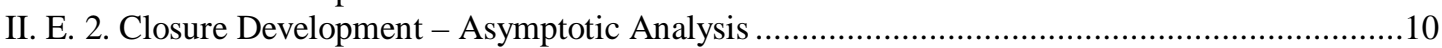

II. E. 3. Closure Development - Desired Diffusion Limit ........................................................ 12

II. E. 4. Closure Development - Conservation on the Dual Grid ............................................... 14

II. E. 5. Closure Development - Capturing the Correct Diffusion Discretization ..............................16

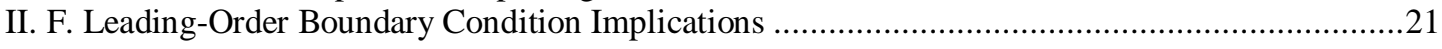

II. G. Closure Development - Improving Performance for Source-Free Purely Absorbing Media.......22

II. H. Source Extrapolations............................................................................................25

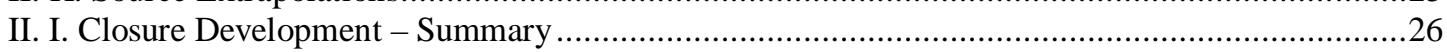

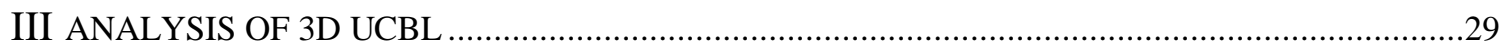

III. A. Three-dimensional Polyhedral Discretization.............................................................29

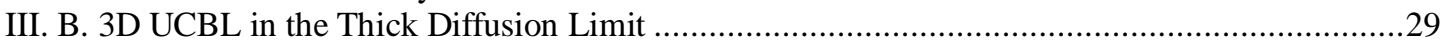

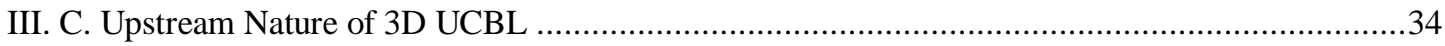

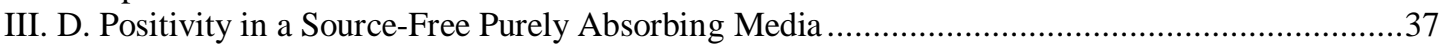

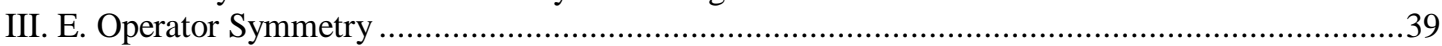

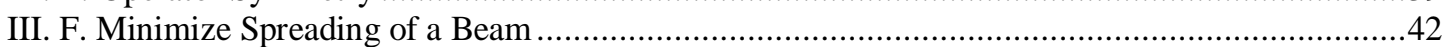

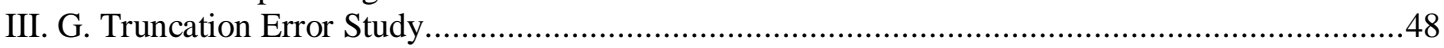

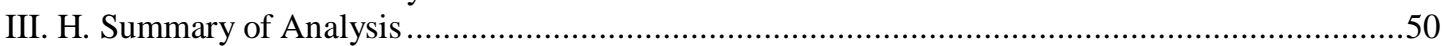


CHAPTER

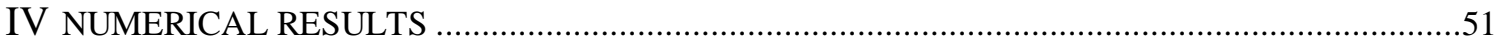

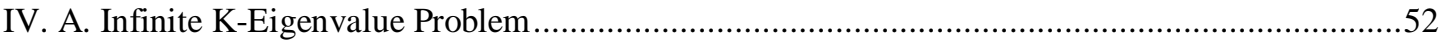

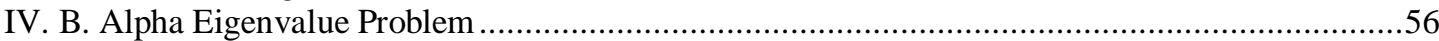

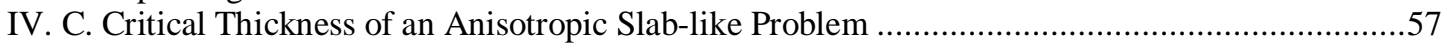

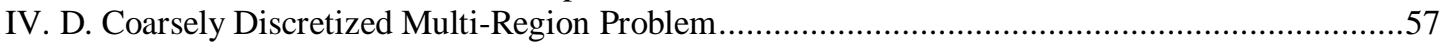

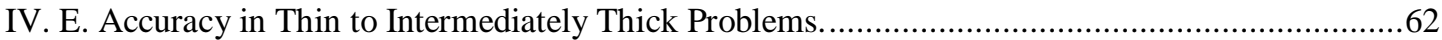

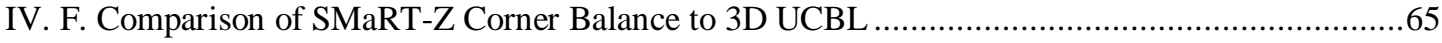

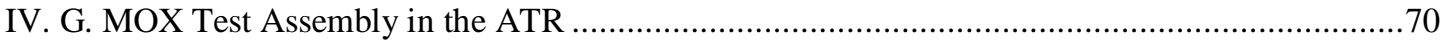

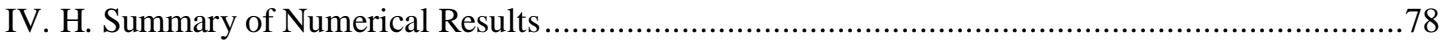

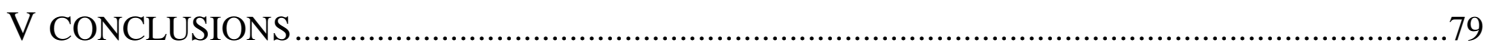

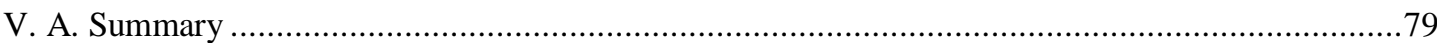

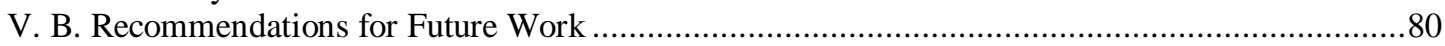

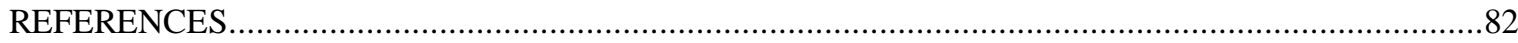

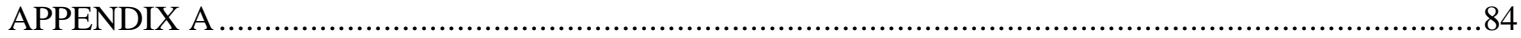

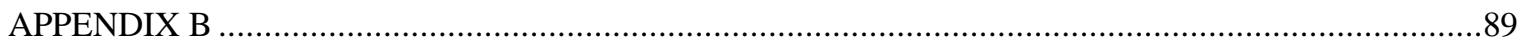

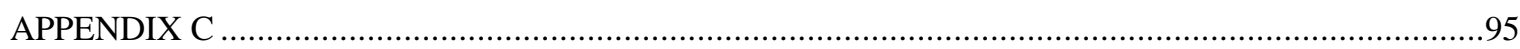

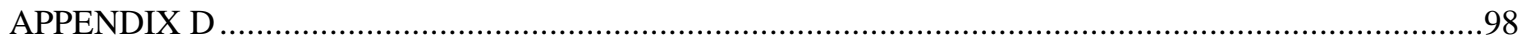

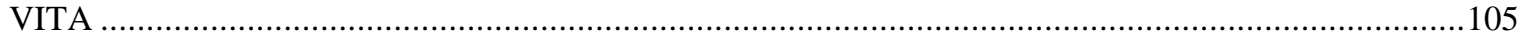




\section{LIST OF FIGURES}

FIGURE

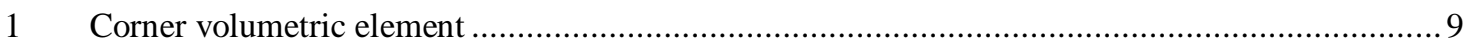

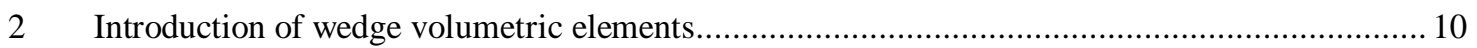

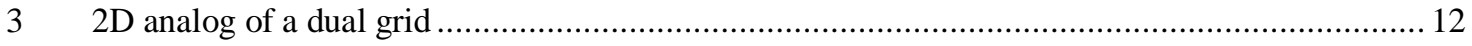

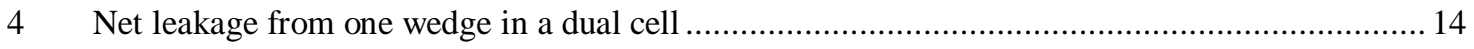

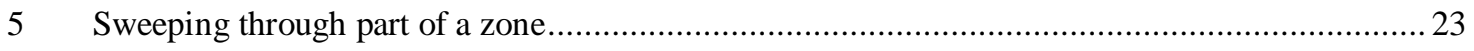

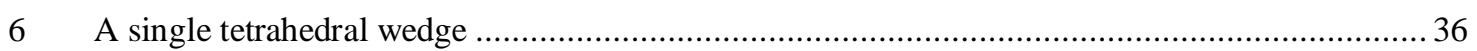

7 An eight-cell grid used to demonstrate the asymmetry of our diffusion operator ......................... 41

8 This example shows how UCBL limits the spreading of beams ................................................. 43

9 Numerical test that demonstrates that 3D UCBL captures exactly the analytic solution for

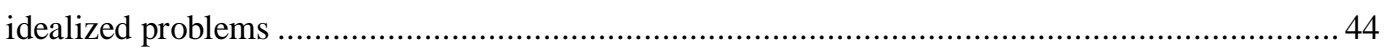

10 Lineout solution for a beam in a vacuum (ideal geometry) ................................................... 45

11 Numerical test that demonstrates that 3D UCBL limits the numerical diffusion of a beam in

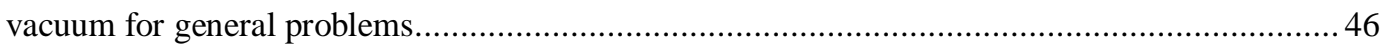

12 Lineout solution for a beam in a vacuum (general geometry) .................................................. 47

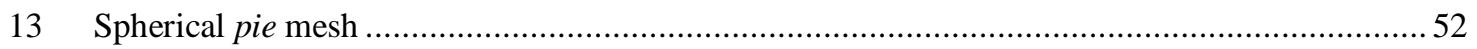

14 The scalar flux solution of the k-eigenvalue reflected sphere problem ......................................5

15 The scalar flux solution of the k-eigenvalue sphere problem with white boundary conditions.......55

16 Geometry and material map for the coarsely discretized multi-region problem ...........................58

17 3D UCBL corner midpoint solution for the coarsely discretized multi-region problem................59

18 3D UCBL cell midpoint solution for the coarsely discretized multi-region problem .................... 60

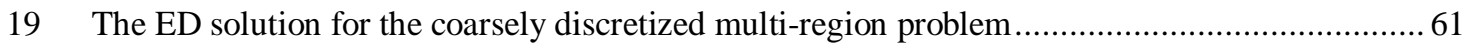

20 The DD solution with fixups solution for the coarsely discretized multi-region problem ............. 62

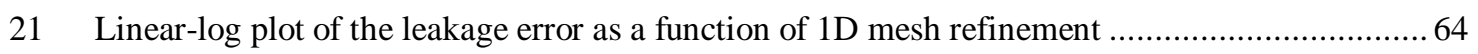

22 Log-log plot of the leakage error as a function of 3D mesh refinement ..................................... 65

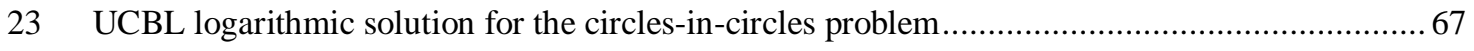

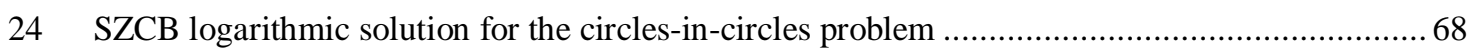

25 Line-outs at $(\mathrm{x}, \mathrm{y})=(2.56,1.47) \mathrm{cm}$ of the UCBL and SZCB solutions

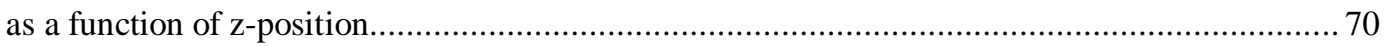

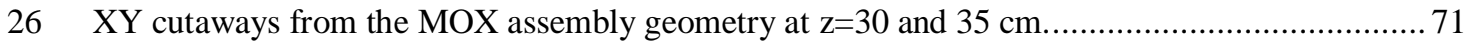

$27 X Y$ cutaways from the MOX assembly geometry at $z=10$ and $29 \mathrm{~cm}$.................................... 72

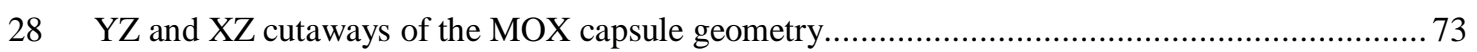

29 UCBL Group 1 Solution for the ATR MOX Assembly Simulation ............................................ 76

30 UCBL Group 2 Solution for the ATR MOX Assembly Simulation ........................................... 77 
FIGURE

31 Calculating the reflected direction of a quadrature direction from an arbitrarily oriented surface... .90

32 Angle of interest and its relation to exact reflected angles that are not in the quadrature set........ 93

33 A rectangular wedge divided into eight wedges......................................................... 98

34 A row of rectangular cells with an incident beam in cell zero. ............................................ 100

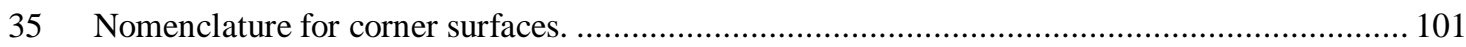

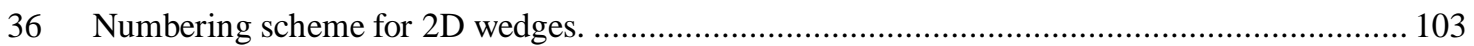




\section{LIST OF TABLES}

TABLE

I Errors in Exiting Flow Rate (EFR) and Absorption Rate (AR) and the Error Reduction Ratio (ERR) for Various Methods and Grids for a Slab Like Problem 48

II Errors in Exiting Flow Rate (EFR) and Absorption Rate (AR) and the Error Reduction Ratio (ERR) for Various Methods and Grids for a 3D Problem

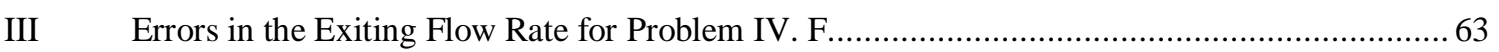

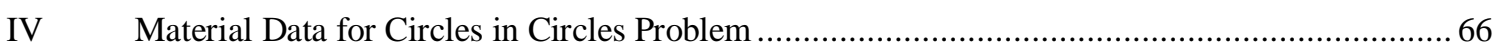

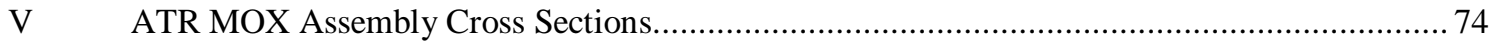




\title{
A Spatial Discretization Scheme for Solving the Transport Equation on Unstructured Grids of Polyhedra
}

\author{
by \\ KELLY GLEN THOMPSON
}

\begin{abstract}
In this work, we develop a new spatial discretization scheme that may be used to numerically solve the neutron transport equation. This new discretization extends the family of corner balance spatial discretizations to include spatial grids of arbitrary polyhedra. This scheme enforces balance on subcell volumes called corners. It produces a lower triangular matrix for sweeping, is algebraically linear, is nonnegative in a source-free absorber, and produces a robust and accurate solution in thick diffusive regions. Using an asymptotic analysis, we design the scheme so that in thick diffusive regions it will attain the same solution as an accurate polyhedral diffusion discretization. We then refine the approximations in the scheme to reduce numerical diffusion in vacuums, and we attempt to capture a second order truncation error.

After we develop this Upstream Corner Balance Linear (UCBL) discretization we analyze its characteristics in several limits. We complete a full diffusion limit analysis showing that we capture the desired diffusion discretization in optically thick and highly scattering media. We review the upstream and linear properties of our discretization and then demonstrate that our scheme captures strictly non-negative solutions in source-free purely absorbing media. We then demonstrate the minimization of numerical diffusion of a beam and then demonstrate that the scheme is, in general, first order accurate. We also note that for slab-like problems our method actually behaves like a second-order method over a range of cell thicknesses that are of practical interest. We also discuss why our scheme is first order accurate for truly 3D problems and suggest changes in the algorithm that should make it a second-order accurate scheme.

Finally, we demonstrate 3D UCBL's performance on several very different test problems. We show good performance in diffusive and streaming problems. We analyze truncation error in a 3D problem and demonstrate robustness in a coarsely discretized problem that contains sharp boundary layers. We also examine eigenvalue and fixed source problems with mixed-shape meshes, anisotropic scattering and multigroup cross sections. Finally, we simulate the MOX fuel assembly in the Advance Test Reactor.
\end{abstract}




\section{CHAPTER I}

\section{INTRODUCTION}

Many scientific and engineering disciplines require a mathematical description of the transport of microscopic particles in a background medium. The transport equation was developed to fill this need, and it is used to solve a variety of problems including nuclear reactor design and analysis, criticality safety, medical therapy and imaging, oil well logging, nuclear weapons safety, and radiative transfer in stellar atmospheres. $^{1-3}$

Transport problems of this nature are solved using either stochastic or deterministic numerical schemes. Because of their geometric flexibility, stochastic methods (e.g. Monte Carlo) have traditionally been used to solve radiative transport problems in complex multidimensional geometries. Until recently, diffusion methods have been implemented where stochastic methods were not viable. The choice to use diffusion solvers has been made primarily because of the large computational cost associated with using more accurate direct discretizations of the transport equation. Unfortunately, diffusion and diffusion-like schemes can be very inaccurate for many types of problems. Recent advances in computing hardware and an increased need for accurate deterministic solutions has stimulated interest in the development and use of deterministic methods for solving multidimensional transport problems. ${ }^{4}$

The most widely used discretization of the direction variable in the transport equation is the discrete ordinates method, in which integrals over direction are replaced by quadrature sums. Currently, there are no publicly available discrete ordinates codes that can solve transport problems on unstructured grids composed of arbitrary polyhedra. When a problem must be solved on such a complex grid, researchers have been forced to employ stochastic methods. Because of the inherent statistical noise found in stochastic methods and the large execution times required for some applications a stochastic solution may not be practical. Additionally, it is desirable to develop deterministic methods that compete with and complement stochastic schemes, thereby giving designers and analysts more tools to use on their problems. We have therefore undertaken the task of developing a scheme for solving the discrete ordinates transport problem on grids of arbitrary polyhedral cells.

There are additional strong arguments for developing a discrete ordinate spatial discretization that use unstructured grids of arbitrary polyhedra. In many applications, other physical processes, such as hydrodynamics, may define the grid. If such an integrated application uses a Lagrangian mesh; then, it is desirable to solve the transport problem on the same mesh that the hydrodynamics code is using. Lagrangian frameworks requires the grid to flow (distort) with the flow of materials. This flow will twist,

This dissertation follows the style and format of Nuclear Science and Engineering. 
distort and may even collapse cells. If the mesh becomes entangled the algorithm must reconnect the cells in the mesh to generate hexahedral cells. While many methods deal with this problem by restricting the mesh to form degenerate hexes, some free Lagrangian hydrodynamics methods utilize the full range of truly arbitrary polyhedra in $3 \mathrm{D} .^{5-6}$

Another reason for using polyhedral grids is the ease of implementing a local mesh refinement algorithm. Typical Adaptive Mesh Refinement, AMR, schemes for non-polyhedral meshes require that specific rules govern the interface between normal cells and refined cells. These rules are needed because the normal mesh will have one face joining to multiple faces of the refined mesh. The unrefined cell in this case looks like a polyhedral cell but can be treated as a much simpler cell shape if these interface rules are in place. However, if our algorithms can use a polyhedral mesh then we can refine the mesh locally without imposing these extra interface restrictions. This feature has increased importance when using AMR in a Lagrangian framework where the interface between normal and refined cells is allowed to morph into multiple individual faces. This type of local refinement can be problematic for a method that can only handle a fixed set of cell types (degenerate hexahedra, tetrahedra only, etc.), but it is not a problem for a method that can deal with arbitrary polyhedra.

The last and strongest argument for studying polyhedral grids is that such grids are the most generic form of spatial discretization, while all other types of grids are restrictive. It may be that these more restrictive grids (tetrahedra only, etc.) are better grids (easier to generate, easier to visualize, faster to sweep, etc.) for most problems. However, we will not be able to demonstrate the advantages of such meshes unless the general case is also studied. Additionally, it may turn out that a discretization scheme constructed for polyhedral meshes may be as accurate and efficient as currently-used discretizations for more limited kinds of unstructured grids, some of which are described in references 3, 7 and 8. If such a discretization can be found, then, the added versatility of a polyhedral mesh would make it preferable to discretizations that limit the complexity of each cell.

The ultimate objective of this work is to develop a discrete ordinates spatial discretization for the transport equation that utilizes unstructured grids composed of arbitrary polyhedra. In addition to this goal, we are interested in creating a scheme that has several other desired properties. We want our scheme to be:

1. Robust and accurate in the thick diffusion limit.

2. Algebraically linear.

3. Strictly conservative on subcell volumes.

4. Purely upstream so that transport sweeps can be completed without inverting matrices for each cell.

Additionally, for thin (neutronics-like problems) our scheme should:

5. Capture strictly non-negative solutions in source-free purely absorbing media.

6. Minimize the artificial spreading of a beam in a vacuum.

7. Minimize the truncation error of the discretization. 
We are particularly interested in how well our new scheme performs in thick diffusive media that are typical for radiative transfer problems. It is important for our scheme to be robust and to accurately limit to a diffusion solution in these regions using a reasonable number of cells. Because of practical limitations, we cannot resolve the spatial variations of the solution on a mean-free path scale so our scheme must be able capture accurate solutions on cells that are hundreds or thousands of mean-free paths thick. To ensure that we capture this property, we will develop our new 3D spatial discretization scheme by building the desired diffusion limit into our conservation and closure equations. We will then add higher-order features to the closures to improve thin-limit behavior. To perform well in the thick limit, our scheme must capture a reasonable diffusion solution. In our case, we will attempt to capture the same solution produced by Palmer's vertex-centered diffusion discretization. ${ }^{9}$ We have chosen to limit to this diffusion discretization because it is defined for polyhedral grids, is second-order accurate and reduces to a standard seven-point scheme on brick grids. Additionally, we plan to develop a corner balance scheme ${ }^{10-11}$ because of its good performance properties in optically thick and diffusive media.

Chapter II of this work details the development of the 3D Upstream Corner Balance Linear (UCBL) scheme including the design of closure equations specifically designed to capture the desired properties in the thick diffusion limit. Because it is important in many applications that particles and energy be conserved, we have forced our scheme to have strict particle conservation on each cell volume and each subcell volume (which we call a "corner"). Methods that have this property are called corner balance, CB, methods. Corner balance methods are well developed and documented for 1D and 2D problems.

We have also found it useful to subdivide our corners (potentially polyhedral volumes) into tetrahedral sub-volumes (which we call wedges) to simplify the sweeping of the mesh. This prevents our computational elements from having re-entrant faces and makes our development simpler since we only have to consider tetrahedral volumes. Our corner and cell volumes may still have re-entrant faces but we avoid the associated sweeping complications by using a wedge based sweeping algorithm. It should be noted that while we sweep a tetrahedral mesh we only solve the transport problem only on corners and cells (i.e. we satisfy a balance equation only on cells and corners).

In the development of the 3D UCBL scheme (consisting of a balance equation plus four closure equations) we have constrained ourselves to a purely algebraically linear system. Most discretizations of the transport equation are linear because the transport equation itself is algebraically linear. However, the spatial discretization scheme does not have to be linear - there exist many nonlinear methods that have good performance characteristics (e.g. exponential discontinuous and methods with negative flux fixups). We have chosen to limit our development to a linear system partially because the analysis of linear schemes is better understood and documented and partly because iterative acceleration tends to be simpler. In addition, the challenge of developing a discretization for polyhedral grids is sufficiently difficult by itself, that it seemed prudent to avoid the complications introduced by nonlinearities. However, in the future, it may be wise to investigate nonlinear schemes. 
The closures developed Chapter II are not only linear but also "upstream." That is, the closure equations contain only known upstream information and source information that is fixed for the transport sweep. The consequence of this requirement is that no matrix inversions are required to solve for the volume-averaged and face-averaged unknowns on each subcell. This enables us to sweep the mesh in a single quadrature direction very efficiently. This type of scheme is not new in this work. Upstream corner balance, UCB, methods have been studied in some detail in $1 \mathrm{D}$ and in $2 \mathrm{D} .{ }^{10-11}$ We capitalize on the success seen in these works by building our system on the 2D UCB framework described by Adams.

After developing the 3D UCBL scheme in Chapter II, we analyze its behavior in certain limits in Chapter III. We start by doing a full diffusion limit analysis ${ }^{11-13}$ to confirm that our scheme does indeed capture the same solution as Palmer's vertex-centered discrete diffusion scheme ${ }^{9}$ in the optically thick and diffusive limit. This is the differencing scheme whose solution 3D UCBL was designed to capture in the thick diffusive limit. We also review the upstream and linear properties of our discretization in Chapter III. The remaining analysis in this chapter focuses on the performance of our scheme in thin nuetronics-like problems. We demonstrate that our method produces strictly non-negative solutions in source-free purely absorbing media. This feature is desirable because negative solutions are not physical, and it is a feature that many discrete ordinates discretizations fail to capture. While our scheme does not guarantee positivity for all problems, it is reassuring to see that it resists negative solutions. This property reduces our susceptibility to the oscillatory solutions for thin problems that plague some discretizations.

In this analysis chapter, we also examine at the order of truncation error and how well our discretization captures a beam in a vacuum. Many schemes promote unphysical diffusion of beams in vacuums. Some even produce undamped oscillatory solutions in voids where there should be an unattenuated beam. ${ }^{14}$ Our scheme mitigates these issues by using area-projection-weighted incident information to compute volumeaveraged solutions. The analysis shown in Chapter III indicates that our approach achieves this goal. The last analysis performed in Chapter III is a truncation-error analysis. We show that our scheme does not perform as well as desired and that this limitation is a result of successfully capturing items (2) and (4) from our list of desired traits. The analysis goes into some detail to explain why our scheme has a lower than desired truncation order.

Chapter IV demonstrates the overall performance characteristics of 3D UCBL by comparing numerical solutions to analytic and numeric benchmarks. We demonstrate performance in a variety of problems. This allows us to point out the strengths and weaknesses of our scheme in a variety of transport regimes. We examine multi-group infinite-medium $\mathrm{k}$ - and alpha-eigenvalue calculations. We show the capture of the analytic solutions for both the eigenvalue and the group-to-group flux ratios. We examine a criticalthickness problem and show that our scheme is successful at capturing the correct eigenvalue in the presence of anisotropic scattering. We follow these eigenvalue calculations with two fixed-source problems. In the first of these problems, we have a very coarsely discretized mesh with regions that have vastly different material properties. This problem creates strong unresolved boundary layers that all methods have difficulty capturing. This allows us to point out some weaknesses in the UCBL method as we compare its solution to 
both Exponential Discontinuous and Diamond Difference solutions. The next fixed-source problem examines general solution quality in a 3D problem and measures truncation error. This set of problems allows us to evaluate the strengths and weaknesses of 3D UCBL including how it compares to other discretizations.

At the end of this chapter we run two additional problems. The first of these is a very crude mockup of a well-logging problem. The geometry of this problem is regular in the z-direction so we will be able to compare our results against SMaRT-Z CB. ${ }^{15}$ This comparison will demonstrate that UCBL not only performs well but also obtains approximately the same solution as other recognized discretizations. In our final test problem we analyze a numerical simulation of the MOX test assembly geometry in the Advanced Test Reactor. This is a detailed real world calculation that should push the limits of our scheme. This is an ideal problem for our scheme because of the difficult geometry (curved surfaces and fine detail). It also has several materials and we demonstrate our ability to solve problems with complex material properties.

Finally, in Chapter V, we offer some concluding remarks and suggestions for future work. We review the strengths and weaknesses of 3D UCBL discovered in Chapters III and IV. We also comment that our scheme does very well in diffusive regions, in thin regions and in intermediate regions but does so at the cost of poor truncation error and large memory requirements. Additionally, the large number of unknowns per cell (48 per angle and group combination) and the current lack of any iterative accelerator means our scheme may require large execution times. We do, however, suggest four modifications that should be investigated to correct these deficiencies. 


\section{CHAPTER II}

\section{DISCRETIZATION DEVELOPMENT}

In this chapter, we revisit the goals of our spatial discretization that are outlined in Chapter I. We introduce the transport equation, the time- energy- and angle-discretizations considered in this work and then proceed to present an overview of asymptotic diffusion analysis. The remainder of this chapter is devoted to developing the system of equations that define our new spatial discretization. These equations will be designed specifically to capture the seven points outlined in Chapter I.

\section{A. Goals for the Discretization}

The primary goal of this development is the construction of a spatial discretization scheme that can utilize an unstructured mesh of polyhedra. There are several other desirable properties that we would like our method to posses as well. We want our method to be robust and accurate in the asymptotic diffusion limit. In fact, for optically thick and highly scattering problems, we would like our scheme to produce solutions that satisfy Palmer's vertex-centered discrete diffusion equation. ${ }^{9}$ We want our method to have strict conservation of particles within cells and corners. We want our method to be algebraically linear, monotonic and positive. It should be fully "upstream" so that we do not need to invert a matrix for each cell. We would like our scheme to preserve certain streaming properties of the transport solution when there is no scattering. Lastly, we would like our discretization to minimize truncation error. It may not be possible to capture all of these characteristics. In the following section we will prioritize these features and attempt to capture as many as possible.

\section{B. The Transport Equation}

We begin our discussion by examining the analytic transport equation since this is the problem we are trying to solve.

$$
\begin{gathered}
\frac{1}{v(E)} \frac{\partial \psi}{\partial t}+\vec{\Omega} \cdot \vec{\nabla} \psi+\sigma_{t}(\vec{r}, E, t) \psi(\vec{r}, \vec{\Omega}, E, t)= \\
\int_{0}^{\infty} d E^{\prime} \int_{4 \pi} d \Omega^{\prime} \sigma_{s}\left(\vec{r}, \vec{\Omega}^{\prime} \rightarrow \vec{\Omega}, E^{\prime} \rightarrow E, t\right) \psi\left(\vec{r}, \vec{\Omega}^{\prime}, E^{\prime}, t\right) \\
+S_{e x t}(\vec{r}, \vec{\Omega}, E, t)+\frac{\chi(E)}{4 \pi} \int_{0}^{\infty} d E^{\prime} v \sigma_{f}\left(\vec{r}, E^{\prime}, t\right) \phi\left(\vec{r}, E^{\prime}, t\right), \\
\phi(\vec{r}, E, t)=\int_{4 \pi} d \Omega^{\prime} \psi\left(\vec{r}, \vec{\Omega}^{\prime}, E, t\right) .
\end{gathered}
$$

On the left we have terms for the rate of change in time, net leakage and absorption. On the right we have terms for inscatter, external sources and fission. The angular flux is represented by $\psi$ and is a function of 
spatial position, $\vec{r}$, direction, $\vec{\Omega}$, energy, $E$, and time, $t$. The particle velocity is specified by $v(E)$ and $\sigma_{t}$ represents the total interaction cross section. $\sigma_{s}$ is the probability that a neutron scatters into the current direction and energy level and $\sigma_{f}$ is the probability that an interaction causes a fission producing $v$ neutrons with energy specified by the function $\chi$. The equation we are focusing on is for neutrons. However, with a few modifications this equation applies to other types of particles including thermal radiation. A detailed development and discussion about the neutron transport equation can be found in references 1 or 2 .

Radiation transport developments may be found in references 16 and in 17.

When solving this system numerically we lump all of the source terms on the right hand side of the equation into a single variable, $Q$. Because the scattering source is a function of the angular flux, equation (2) must be solved iteratively. In typical iterative schemes the information represented by $Q$ is formed from previous iterate values.

$$
\frac{1}{v(E)} \frac{\partial \psi}{\partial t}+\vec{\Omega} \cdot \vec{\nabla} \psi+\sigma_{t}(\vec{r}, E, t) \psi(\vec{r}, \vec{\Omega}, E, t)=Q(\vec{r}, \vec{\Omega}, E, t)
$$

Discretizing the time variable produces a sequence of steady-state problems that will be the basis of our development. Additionally, since our discussion is concerned with the spatial discretization we will not discuss the merits of discrete ordinates angular discretization or multi-group energy discretization that we have chosen to use. The discrete ordinates and multi-group approximations are well understood and have been documented elsewhere. ${ }^{1}$ Our development will start with the energy- and angle-discretized transport equation.

$$
\begin{aligned}
\vec{\Omega}_{m} \cdot \vec{\nabla} \psi_{m}+\sigma_{t, g}(\vec{r}) \psi_{g}\left(\vec{r}, \vec{\Omega}_{m}\right) & =Q_{g}\left(\vec{r}, \vec{\Omega}_{m}\right), \\
Q_{g}\left(\vec{r}, \vec{\Omega}_{m}\right) & =\sum_{g^{\prime}} \sum_{m^{\prime}} w_{m^{\prime}} \sigma_{s, g^{\prime} \rightarrow g}\left(\vec{r}, \vec{\Omega}_{m^{\prime}} \rightarrow \vec{\Omega}_{m}\right) \psi_{g^{\prime}}\left(\vec{r}, \vec{\Omega}_{m^{\prime}}\right) \\
& +S_{e x t, g}\left(\vec{r}, \vec{\Omega}_{m}\right)+\frac{\chi_{g}}{4 \pi} \sum_{g^{\prime}} v \sigma_{f, g^{\prime}}(\vec{r}) \phi_{g^{\prime}}(\vec{r}), \\
\phi_{g}(\vec{r}) & =\sum_{m} w_{m} \psi_{g}\left(\vec{r}, \vec{\Omega}_{m}\right) .
\end{aligned}
$$

It is customary to drop the discrete arguments in favor of subscripting the main variables with the correct index. This is the form of the transport equation that we are interested in solving.

$$
\begin{aligned}
\vec{\Omega}_{m} \cdot \vec{\nabla} \psi_{m, g}+\sigma_{t, g}(\vec{r}) \psi_{m, g}(\vec{r}) & =Q_{m, g}(\vec{r}), \\
Q_{m, g}(\vec{r}) & =\sum_{g^{\prime}} \sum_{m^{\prime}} w_{m^{\prime}} \sigma_{s, m^{\prime} \rightarrow m, g^{\prime} \rightarrow g}(\vec{r}) \psi_{m^{\prime}, g^{\prime}}(\vec{r}) \\
& +S_{m, g}^{e x t}(\vec{r})+\frac{\chi_{g}}{4 \pi} \sum_{g^{\prime}} v \sigma_{f, g^{\prime}}(\vec{r}) \phi_{g^{\prime}}(\vec{r}), \\
\phi_{g}(\vec{r}) & =\sum_{m} w_{m} \psi_{m, g}(\vec{r}) .
\end{aligned}
$$




\section{C. Particle Balance}

We now must discuss spatial discretization. All numerical methods discretize spatially by breaking the problem volume into spatial cells. We will assume that these computational cells are polyhedra. If we integrate equation (4) over the volume of one of these cells and utilize Green's Theorem to cast the gradient term as a surface integral for the cell we arrive at a balance equation for the cell.

$$
\sum_{f \in \text { cell }} \vec{A}_{f} \cdot \vec{\Omega}_{m} \psi_{m, f}+V_{z} \sigma_{t, z} \psi_{m, z}=V_{z} Q_{m, z}
$$

The $\vec{A}_{f}$ are the outward pointing area vectors for each face of the polyhedral cell. We cannot explicitly write down each of the face terms because we do not know how many faces there will be. The terms $\psi_{m, z}$ and $Q_{m, z}$ represent volumetric cell-average quantities for the angular flux and the total source. $V_{z}$ represents the volume of the cell of interest and the $\psi_{m, f}$ terms are surface-averaged angular fluxes that exist on each face of the cell. The total cross section for this volume is expressed by $\sigma_{t, z}$. For the remainder of this work we assume that all material data is constant within each cell volume (i.e. all subcells within this volume will have identical material data). Equation (5) is an expression of balance over a cell and holds for every cell in the problem.

Since we are developing a corner balance method, our discretization will also preserve balance on corners. A corner is a sub-cell volume. There is one corner associated with each vertex in a cell. This vertex, the cell volumetric midpoint, all cell face midpoints adjacent to the vertex of interest and all edge midpoints adjacent to the vertex define a corner, as shown in Figure 1. An edge point is always the midpoint between two vertices of a cell. We require particle balance on every corner in the problem. This requirement can be expressed with another balance equation.

$$
\sum_{f_{c} \in \text { corner }} \vec{A}_{f_{c}} \cdot \vec{\Omega}_{m} \psi_{m, f_{c}}+V_{c} \sigma_{t} \psi_{m, c}=V_{c} Q_{m, c}
$$

The $\vec{A}_{f_{c}}$ are outward pointing area vectors for each corner face. The terms $\psi_{m, c}$ and $Q_{m, c}$ represent volumetric corner-average quantities for the angular flux and the total source. The face average angular fluxes are represented by $\psi_{m, f_{c}}$. 


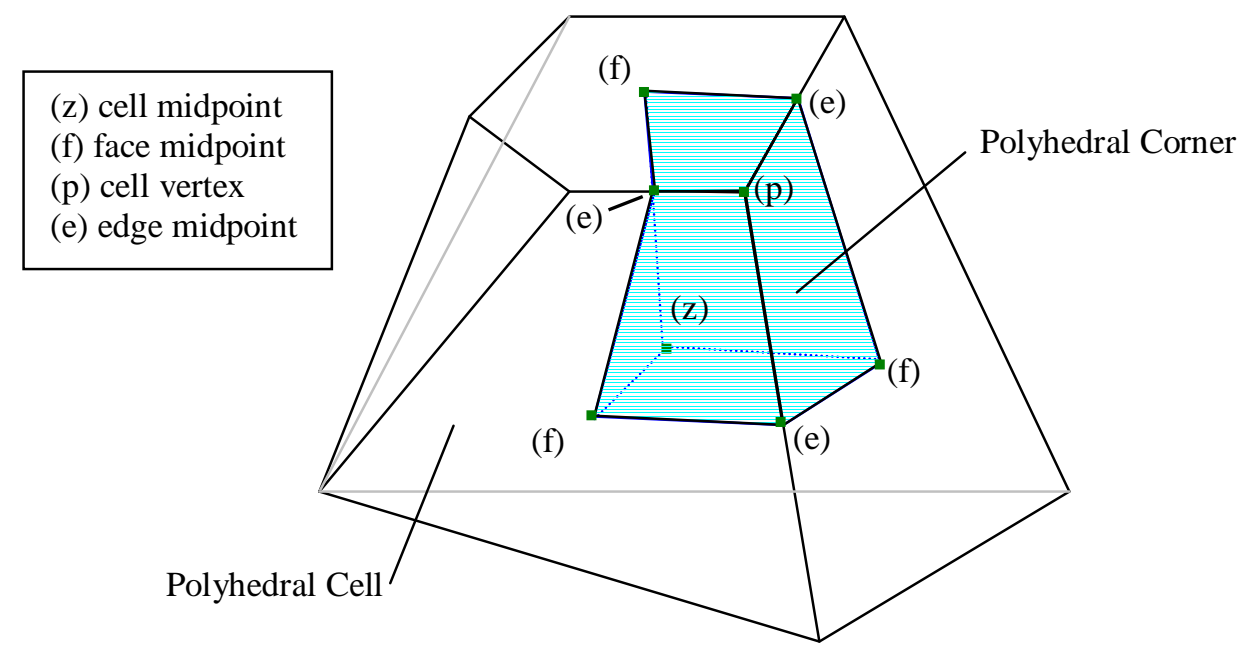

Figure 1. Corner volumetric element.

\section{D. Wedges}

It is difficult to develop a consistent sweeping algorithm for polyhedral elements so we have chosen to break our corners into sub-corner tetrahedral elements called wedges. A cell vertex, a cell volumetric midpoint, a face midpoint and an edge point define a wedge as shown in Figure 2. We will not enforce particle balance on wedges but we will have a pseudo-balance equation for each wedge.

$$
\begin{aligned}
& \vec{A}_{f e z, w} \cdot \vec{\Omega}_{m} \psi_{m, f e z}+\vec{A}_{f e p, w} \cdot \vec{\Omega}_{m} \psi_{m, f e p}+\vec{A}_{p e z, w} \cdot \vec{\Omega}_{m} \psi_{m, p e z} \\
& +\vec{A}_{f p z, w} \cdot \vec{\Omega}_{m} \psi_{m, f p z}+V_{w} \sigma_{t} \psi_{m, w}=V_{w} Q_{m, c} .
\end{aligned}
$$

This is not a true balance equation because the total source $Q_{m, c}$ is the volumetric average for the whole corner and not just the wedge. The area vectors and face averaged angular fluxes are specified for each of the four tetrahedral faces: fez, fep, fep and fep. It should be noted that we could sum equation (7) for all wedges in a corner or all wedges in a cell to arrive at equations (6) or (5) respectively. 
(f)

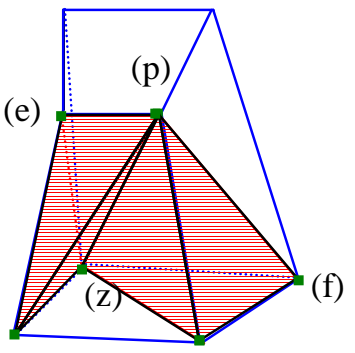

(e)

Polyhedral Corner

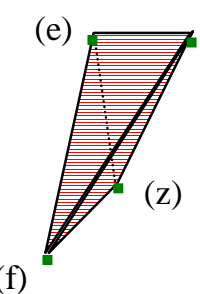

(f)

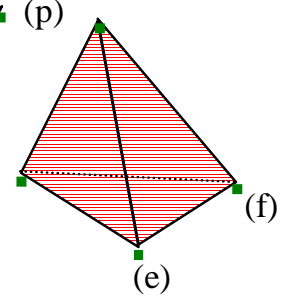

\section{(z) cell center \\ (f) face midpoint \\ (p) cell vertex \\ (e) edge midpoint}

Tetrahedral Wedges

Figure 2. Introduction of wedge volumetric elements.

\section{E. Closure Development}

\section{E. 1. Closure Development - Introduction}

Our spatial discretization scheme is defined by our wedge balance equation (which insures particle balance on corners and cells) in conjunction with four closure equations that relate the unknown surfaceaveraged intensities to wedge-average intensities. We will develop these closures in such a way that we satisfy as many as possible of the goals listed in Section II.A. Since we are solving the transport equation on wedges that make up polyhedral corners and zones we have already satisfied our first goal. As the name of our discretization implies we must ensure that all of our closure equations are upstream. Third in importance is our ability to capture a vertex-centered discrete diffusion solution. The development of our closures will be based primarily on the latter two requirements. In the following section we will use a diffusion-limit analysis to build our closure equations.

\section{E. 2. Closure Development-Asymptotic Analysis}

Diffusion limit analysis, DLA, is typically used to assess a spatial discretization scheme's behavior when the problem of interest becomes optically thick and highly scattering. This analysis mathematically relates diffusion theory to transport theory by making use of asymptotic expansion of unknowns and a parameterized scaling of the transport equation. In particular, this analysis can predict if the leading-order term of the asymptotic expansion satisfies a reasonable diffusion discretization in a thick diffusive problem. If the leading order transport solution does not satisfy a reasonable diffusion discretization then it will not be accurate in thick diffusive problems. This asymptotic analysis can also predict how robust and accurate a method will be in problems that are near but not quite diffusive. Below we will use a DLA to build our closure equations so that our scheme remains robust and captures an accurate solution in diffusive problems. 
For this analysis we will assume that the scattering cross section is isotropic so that it can be expanded into the difference between the total and the absorption cross sections. We will also lump the fission term into $S_{\text {ext }}$ and assume that any other sources are isotropic so the RHS of our transport equation takes on a simpler form.

$$
Q_{m}(\vec{r})=\frac{1}{4 \pi} S_{0}^{\text {ext }}(\vec{r})+\frac{1}{4 \pi}\left[\sigma_{s}(\vec{r})-\sigma_{a}(\vec{r})\right] \phi(\vec{r}) .
$$

For the multi-group approximation we have assumed that the transport equation can be discretized in energy to produce a series of mono-energetic equation. In this development we only consider this mono-energetic form of the transport equation.

DLA is an asymptotic expansion of the fundamental transport unknowns (i.e. angular flux) combined with a scaling of the transport equation (and closures) with a small parameter, $\varepsilon$, so that as $\mathcal{E} \rightarrow 0$ our system becomes optically thick and highly scattering. We choose the following scaling of the analytic transport equation. (Adams, 97)

$$
\begin{aligned}
\vec{\Omega}_{m} \cdot \vec{\nabla} \psi_{m}+\frac{\sigma_{t}(\vec{r})}{\varepsilon} \psi_{m}(\vec{r}) & =\frac{\varepsilon}{4 \pi} S_{0}^{e x t}(\vec{r})+\frac{1}{4 \pi}\left[\frac{\sigma_{t}(\vec{r})}{\varepsilon}-\varepsilon \sigma_{a}(\vec{r})\right] \phi(\vec{r}), \\
\phi(\vec{r}) & =\sum_{m} w_{m} \psi_{m}(\vec{r}) .
\end{aligned}
$$

Next, we will postulate that the solution is a power series in $\varepsilon$.

$$
\begin{aligned}
\psi_{m} & =\psi_{m}^{(0)}+\varepsilon \psi_{m}^{(1)}+\varepsilon^{2} \psi_{m}^{(2)}+\ldots, \\
\phi & =\phi^{(0)}+\varepsilon \phi^{(1)}+\varepsilon^{2} \phi^{(2)}+\ldots
\end{aligned}
$$

By examining the $O(1 / \mathcal{E})$ terms in our scaled equation to we find that the leading order angular flux is isotropic in the interior of the problem. Using both leading order and $O(1)$ information it can be shown that the leading order angular flux satisfies a diffusion equation.

$$
\begin{gathered}
\psi_{m}^{(0)}(\vec{r})=\frac{1}{4 \pi} \phi^{(0)}, \\
\vec{J}^{(1)}(\vec{r})=\sum_{m} w_{m} \vec{\Omega}_{m} \psi_{m}^{(1)}(\vec{r})=\frac{-1}{3 \sigma_{t}(\vec{r})} \vec{\nabla} \phi^{(0)}(\vec{r}), \\
\vec{\nabla} \cdot \vec{J}^{(1)}(\vec{r})+\sigma_{a}(\vec{r}) \phi^{(0)}(\vec{r})=S_{0}^{\text {ext }}(\vec{r}) .
\end{gathered}
$$

To complete this description we must also prescribe a form for the leading-order solution on the boundary of the problem. Malvagi and Pomraning have presented the following form for the analytic boundary condition. ${ }^{18}$

$$
\phi^{(0)}(\vec{r})=2 \int_{\vec{n} \cdot \vec{\Omega}<0} d \Omega W(|\vec{n} \cdot \vec{\Omega}|) \psi^{i n c}(\vec{r}, \vec{\Omega}), \quad \vec{r} \in \Gamma .
$$

In this equation we are examining only the leading-order flux on the boundary of the problem $(\vec{r} \in \Gamma)$. The function $W$ is defined in terms of Chandrasekhar's $H$-function ${ }^{19}$ for a purely scattering medium. This function may be approximated by a simple polynomial. ${ }^{11}$

$$
\begin{aligned}
W(\mu) & =\frac{\sqrt{3}}{2} H(\mu), \\
& =0.91 \mu+1.635 \mu^{2} \pm a \text { few } \%, \\
& \approx \mu+\frac{3}{2} \mu^{2} .
\end{aligned}
$$




\section{E. 3. Closure Development - Desired Diffusion Limit}

In the above section we have shown that in the thick diffusion limit the exact discrete ordinates angular discretization captures a diffusion solution to leading order. However, we are looking for a set of equations that produce, to leading order in a thick diffusive region, a solution of Palmer's vertex-centered diffusion discretization. ${ }^{9}$ To capture this form we must require our scheme to (1) obtain conservation on a dual grid and (2) capture exactly the same leakage on the surface of this dual grid as Palmer's scheme.

Our dual grid is a grid formed by the union of all corners sharing a common vertex. This union of corners forms the same control volume used by the discrete diffusion system we are attempting to limit to. It is difficult to visualize this dual grid in 3D. However its $2 \mathrm{D}$ analog is shown in Figure 3.
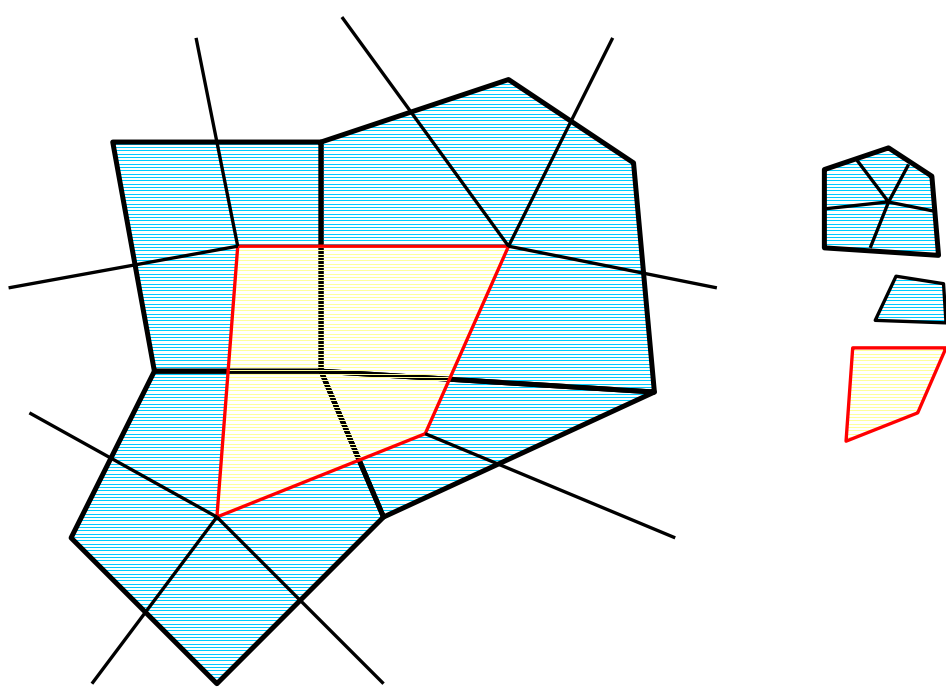

Cell with corners outlined

Corner

Dual grid cell

(all corners about a common vertex)

Figure 3. 2D analog of a dual grid.

Palmer requires the net current across each facet of his control volume to be expressed in terms of point fluxes. The point scalar fluxes, $\phi_{p}, \phi_{z}, \phi_{e}$ and $\phi_{f}$, live at the vertices of each wedge. The subscripts stand for point, zone (cell), edge and face vertices as shown in Figure 2. Palmer's expression is

$$
\vec{A}_{f e z, w} \cdot \vec{J}_{f e z, w}=\frac{1}{9 \sigma_{t} V_{w}} \vec{A}_{f e z, w} \cdot\left(\vec{A}_{f e z, w} \phi_{p}+\vec{A}_{f e p, w} \phi_{z}+\vec{A}_{f p z, w} \phi_{e}+\vec{A}_{f e z, w} \phi_{f}\right) .
$$

In this expression each of the area vectors is assumed to point out from the fepz control volume. Palmer further defines the zone-center fluxes in terms of point- and face-center fluxes using a zone-averaged gradient. 


$$
\phi_{z}=\sum_{w \in z} w_{w}\left(\phi_{p}+\vec{l}_{p z} \cdot\langle\vec{\nabla} \phi\rangle_{z}\right), \quad w_{w}=\frac{\frac{1}{\left|\vec{l}_{p z}\right|}}{\sum_{w \in z} \frac{1}{\left|\vec{l}_{p z}\right|}}, \quad\langle\vec{\nabla} \phi\rangle_{z}=\frac{1}{V_{w}} \sum_{f \in z} \vec{A}_{f} \phi_{f} .
$$

Here $\vec{A}_{f}$ is the outward pointing area vector for each face of the control volume and $\vec{l}_{p z}$ is the vector between the zone-center and a vertex. The notation $w \in z$ should be read as all wedges that are members of zone $z$. The face-centered flux is defined similarly.

$$
\phi_{f}=\sum_{w \in f} w_{w}\left(\phi_{p}+\vec{l}_{p f} \cdot\langle\vec{\nabla} \phi\rangle_{f}\right), \quad w_{w}=\frac{\frac{1}{\left|\vec{l}_{p f}\right|}}{\sum_{w \in f} \frac{1}{\left|\vec{l}_{p f}\right|}}, \quad\langle\vec{\nabla} \phi\rangle_{f}=\frac{1}{A_{f}} \sum_{w \in f} \vec{l}_{p e} \phi_{p} .
$$

The edge scalar flux is defined to be the linear average of the two point fluxes that it lies between as shown in Figure 4.

$$
\phi_{e}=\frac{1}{2}\left(\phi_{p}+\phi_{p^{\prime}}\right) \text {. }
$$

These equations completely define the diffusion discretization proposed by Palmer. However, we can write equation (14) in a more useful form by performing some additional algebra.

$$
\vec{A}_{f e z, w} \cdot \vec{J}_{f e z, w}=\frac{-1}{6 \sigma_{t} V_{w}} \vec{A}_{f e z, w} \cdot\left[\begin{array}{c}
\frac{1}{3} \vec{A}_{f e p, w}\left(\phi_{f}+\phi_{e}+\phi_{p}\right)+\frac{1}{3} \vec{A}_{f e p^{\prime}, w}\left(\phi_{f}+\phi_{e}+\phi_{p^{\prime}}\right) \\
+\frac{1}{3} \vec{A}_{p e z, w}\left(\phi_{p}+\phi_{e}+\phi_{z}\right)+\frac{1}{3} \vec{A}_{p^{\prime} e z, w}\left(\phi_{p^{\prime}}+\phi_{e}+\phi_{z}\right) \\
+\frac{1}{3} \vec{A}_{f p z, w}\left(\phi_{f}+\phi_{p}+\phi_{z}\right)+\frac{1}{3} \vec{A}_{f p^{\prime} z, w}\left(\phi_{f}+\phi_{p^{\prime}}+\phi_{z}\right)
\end{array}\right] .
$$

The $p^{\prime}$ notation refers to the zone vertex of the wedge that shares the fez face with the current wedge as shown in Figure 4. 


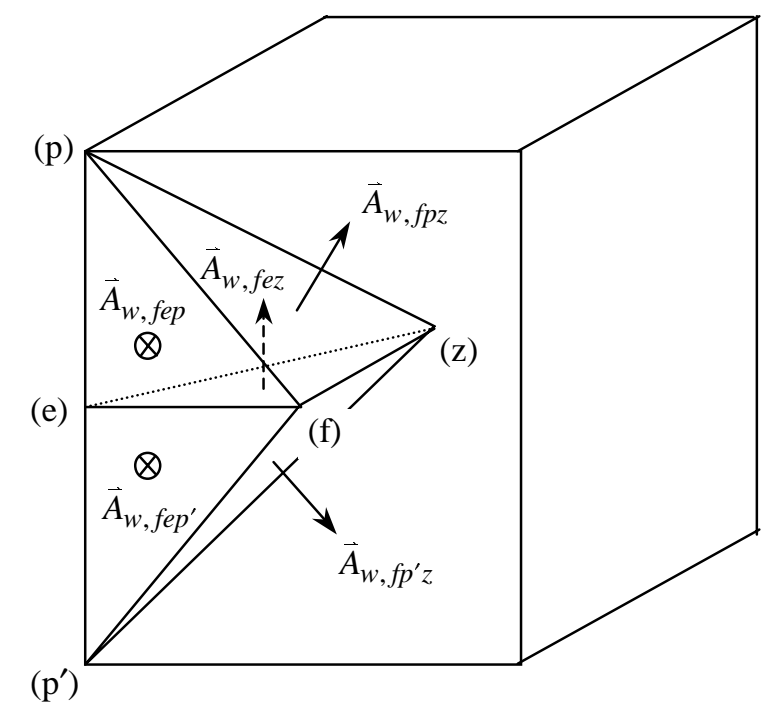

Figure 4. Net leakage from one wedge in a dual cell.

\section{E. 4. Closure Development-Conservation on the Dual Grid}

If we are to limit to a vertex-centered diffusion discretization our method will have to preserve balance to leading order the diffusion solution on a dual grid. In the dual grid the vertices of the primary grid are now volumetric midpoints as shown in Figure 3. The union of all the corner volumes that share a common vertex constitute a dual grid cell. The vertex-centered diffusion discretization that we want to limit to has one degree of freedom (once scalar flux unknown) per vertex where our corner-based scheme has $\mathrm{N}$ degrees of freedom ( $\mathrm{N}$ being the number of corners at the vertex). Previous $\mathrm{CB}$ methods have used a step closure on cell boundaries, which has ultimately forced their leading-order corner fluxes around a vertex to be equal in the diffusion limit. This mechanism limits CB schemes to a single unknown per vertex and we have chosen to use this same procedure in our development of UCBL. We now examine our wedge pseudo-balance equation with asymptotic scaling to demonstrate that our leading-order corner solution is isotropic and equal for all corners in each dual cell.

$$
\begin{aligned}
& \vec{A}_{f e z, w} \cdot \vec{\Omega}_{m} \psi_{m, f e z}+\vec{A}_{f e p, w} \cdot \vec{\Omega}_{m} \psi_{m, f e p}+\vec{A}_{p e z, w} \cdot \vec{\Omega}_{m} \psi_{m, p e z} \\
& \quad+\vec{A}_{f p z, w} \cdot \vec{\Omega}_{m} \psi_{m, f p z}+V_{w} \frac{\sigma_{t}}{\varepsilon} \psi_{m, w}=V_{w}\left[\frac{1}{4 \pi}\left(\frac{\sigma_{t}}{\varepsilon}-\varepsilon \sigma_{a}\right) \phi_{c}+\frac{\varepsilon}{4 \pi} S_{0}^{e x t}\right] .
\end{aligned}
$$

Using the asymptotic ansatz of equation (10) for each discrete unknown and examining the leading order solution ( $1 / \mathcal{E}$ terms), we the wedge-averaged angular flux is isotropic and equal for all the wedges in a single corner.

$$
\psi_{m, w}^{(0)}=\frac{1}{4 \pi} \phi_{c}^{(0)}
$$


Next we write the $O(1)$ wedge pseudo-balance equation.

$$
\begin{gathered}
\vec{A}_{f e z, w} \cdot \vec{\Omega}_{m} \psi_{m, f e z}^{(0)}+\vec{A}_{f e p, w} \cdot \vec{\Omega}_{m} \psi_{m, f e p}^{(0)}+\vec{A}_{p e z, w} \cdot \vec{\Omega}_{m} \psi_{m, p e z}^{(0)} \\
+\vec{A}_{f p z, w} \cdot \vec{\Omega}_{m} \psi_{m, f p z}^{(0)}+V_{w} \sigma_{t} \psi_{m, w}^{(1)}=\frac{1}{4 \pi} V_{w} \sigma_{t} \phi_{c}^{(1)} .
\end{gathered}
$$

We can arrive at an expression for the $O(1)$ corner-balance equation by summing this expression over all wedges in a corner.

$$
\sum_{w \in c}\left[\vec{A}_{f e z, w} \cdot \vec{\Omega}_{m} \psi_{m, f e z}^{(0)}+\vec{A}_{f e p, w} \cdot \vec{\Omega}_{m} \psi_{m, f e p}^{(0)}\right]+V_{c} \sigma_{t} \sum_{w \in c} \psi_{m, w}^{(1)}=\frac{1}{4 \pi} V_{c} \sigma_{t} \phi_{c}^{(1)} .
$$

We note that the $p e z$ and $f p z$ terms will cancel out when we evaluate balance for the whole corner. We can now integrate our leading-order balance equation over all angles. This allows the last two terms to cancel leaving us with an expression that essentially states that the net current flow across the corner will be zero to leading order.

$$
\sum_{w \in c} \sum_{m} w_{m}\left[\vec{A}_{f e z, w} \cdot \vec{\Omega}_{m} \psi_{m, f e z}^{(0)}+\vec{A}_{f e p, w} \cdot \vec{\Omega}_{m} \psi_{m, f e p}^{(0)}\right]=0 .
$$

Remember that to leading order there is no absorption and no volumetric source so the net current should indeed be zero.

From previous work in 2D, we know that to leading order our angular flux must be isotropic and that we want to use a step closure for $\psi_{w, \text { fep }}$. Following the work of 2D UCB closures we will propose the following closure for the $f e p$ surface intensity.

$$
\psi_{m, f e p}= \begin{cases}\psi_{m, w} & \vec{A}_{f e p, w} \cdot \vec{\Omega}_{m}>0, \\ \psi_{m, \tilde{w}_{f e p}} & \vec{A}_{\text {fep }, w} \cdot \vec{\Omega}_{m}<0, \quad \vec{r} \notin \Gamma, \\ \psi_{m, \text { fep }}^{i n c} & \vec{A}_{\text {fep }, w} \cdot \vec{\Omega}_{m}<0, \quad \vec{r} \in \Gamma .\end{cases}
$$

In this closure, $\tilde{w}_{f e p}$ is the wedge that shares the $f e p$ face with wedge $w$. We also assume that the angular flux on the boundary of the problem, $\Gamma$, is specified. We note that the wedge-averaged angular flux is isotropic to leading order so that our fep surface angular flux will be half-range isotropic to leading order in the interior of the problem.

$$
\psi_{m, f e p}^{(0)}= \begin{cases}\frac{1}{4 \pi} \phi_{c}^{(0)} & \vec{A}_{f e p, w} \cdot \vec{\Omega}_{m}>0, \\ \frac{1}{4 \pi} \phi_{\tilde{c}_{f e p}}^{(0)} & \vec{A}_{f e p, w} \cdot \vec{\Omega}_{m}<0, \quad \vec{r} \notin \Gamma, \\ \psi_{m, \text { fep }}^{i n c,(0)} & \vec{A}_{f e p, w} \cdot \vec{\Omega}_{m}<0, \quad \vec{r} \in \Gamma .\end{cases}
$$

Note that as before we let $\tilde{c}_{f e p}$ represent the corner that shares the fep face with wedge $w$ while $c$ is the corner that contains wedge $w$. It should be noted that $\tilde{c}_{f e p}$ is not contained in the same cell as $c$. Again based on previous 2D work, we will require that to leading order the remaining three surface-averaged angular fluxes be isotropic to leading order so that when we integrate over all angles these terms in equation (23) will drop out. For example, if $\psi_{m, f e z}$ is isotropic to leading order then we have

$$
\vec{A}_{f e z, w} \cdot \sum_{m} w_{m} \vec{\Omega}_{m} \psi_{m, f e z}^{(0)} \rightarrow \widetilde{\phi}_{m, f e z}^{(0)} \vec{A}_{f e z, w} \cdot \sum_{m} w_{m} \vec{\Omega}_{m} \frac{1}{4 \pi}=0 .
$$


Later we will devise closures that guarantee this for those three faces. Note also that we assume here (and for the remainder of this work) that our quadrature set correctly integrates $\vec{\Omega}_{m}$ over all angles.

$$
\sum_{m} w_{m} \vec{\Omega}_{m}=0
$$

This leaves us with a very simple balance equation.

$$
\begin{aligned}
0 & =\sum_{w \in c} \vec{A}_{f e p, w} \cdot \sum_{m} w_{m} \vec{\Omega}_{m} \psi_{m, f e p}^{(0)}, \\
& =\sum_{w \in c} \vec{A}_{f e p, w} \cdot\left[\sum_{\vec{A}_{f e p, w} \cdot \vec{\Omega}_{m}>0} w_{m} \vec{\Omega}_{m} \phi_{c}^{(0)}+\sum_{\vec{A}_{f e p, w} \cdot \vec{\Omega}_{m}<0} w_{m} \vec{\Omega}_{m} \phi_{\tilde{c}_{f e p}}^{(0)}\right], \\
& =\sum_{w \in c}\left(\phi_{c}^{(0)}-\phi_{\tilde{c}_{f e p}}^{(0)}\right) \vec{A}_{f e p, w} \cdot \sum_{\vec{A}_{f e p, w} \cdot \sum_{m}>0} w_{m} \vec{\Omega}_{m} .
\end{aligned}
$$

The last line assumes that our quadrature set is symmetric. The consequence of equation (28) is that the leading-order corner-averaged fluxes in the interior of the problem are equal for all corners surrounding a common vertex

$$
\phi_{c}^{(0)}=\phi_{\tilde{c}_{\text {fep }}}^{(0)} \equiv \phi_{p}^{(0)} \quad \forall c, \tilde{c}_{f e p} \in p .
$$

The details leading to this conclusion are discussed in Appendix A.

In summary, our choice for the fep closure means that the leading-order fluxes in all the corners about a common vertex are isotropic and equal. This feature allows UCBL to limit to the vertex-centered discrete diffusion solution discussed above.

\section{E. 5. Closure Development - Capturing the Correct Diffusion Discretization}

The remaining three closures are designed to meet our requirement to limit to Palmer's expression for the net current across the $f e z$ face from equation (18). We start by assuming that the $f e z$ closure has three terms.

$$
\psi_{m, f e z}= \begin{cases}{\left[T_{1}\right]_{c \rightarrow \tilde{c}_{f e z}}+\left[T_{2}\right]_{c \rightarrow \tilde{c}_{f e z}}+\left[T_{3}\right]_{c \rightarrow \tilde{c}_{f e z}},} & \vec{A}_{f e z, w} \cdot \vec{\Omega}>0, \\ {\left[T_{1}\right]_{\tilde{c}_{f e z} \rightarrow c}+\left[T_{2}\right]_{\tilde{c}_{f e z} \rightarrow c}+\left[T_{3}\right]_{\tilde{c}_{f e z} \rightarrow c},} & \vec{A}_{f e z, w} \cdot \vec{\Omega}<0 .\end{cases}
$$

To leading order we will require $\left[T_{1}\right]$ to be isotropic and $\left[T_{2}\right]=\left[T_{3}\right]=0$. This satisfies our requirement for the $f e z$ closure to be isotropic to leading order. We will require $\left[T_{3}\right]=0$ to $O(\mathcal{E})$ so that $\left[T_{2}\right]$ can be formulated to achieve the correct current across the $f e z$ face. $\left[T_{3}\right]$ is a higher order term that can be used to improve our method's performance in other limits. In 2D this term is also used to improve the solution near boundaries and we will discuss this later for our 3D scheme. 
When considering a form for $\left[T_{l}\right]$ we again lean heavily on what has been done in $2 \mathrm{D}$ UCB schemes. In these schemes the following form has been used.

$$
\begin{aligned}
& {\left[T_{1}\right]_{c \rightarrow \tilde{c}_{f e z}}=\psi_{m, w}+\frac{1}{2}\left(\left.\frac{Q_{m}}{\sigma_{t}}\right|_{\tilde{c}_{f e z}}-\left.\frac{Q_{m}}{\sigma_{t}}\right|_{c}\right),} \\
& {\left[T_{1}\right]_{\tilde{c}_{f e z} \rightarrow c}=\psi_{m, \tilde{w}_{f e z}}+\frac{1}{2}\left(\left.\frac{Q_{m}}{\sigma_{t}}\right|_{c}-\left.\frac{Q_{m}}{\sigma_{t}}\right|_{\tilde{c}_{f e z}}\right) .}
\end{aligned}
$$

The source over sigma term may be written using our thick diffusion limit (TDL) scaling.

$$
\left.\frac{Q_{m}}{\sigma_{t}}\right|_{c} \rightarrow \frac{\varepsilon^{2}}{4 \pi \sigma_{t}} S_{0}^{e x t}+\frac{1}{4 \pi}\left[1-\frac{\varepsilon^{2} \sigma_{a}}{\sigma_{t}}\right]\left(\phi_{c}^{(0)}+\varepsilon \phi_{c}^{(1)}+\varepsilon^{2} \phi_{c}^{(2)}+\ldots\right) .
$$

Using this scaling and equation (20) we see that term 1 is indeed isotropic to leading order:

$$
\left[T_{1}\right]^{(0)}=\psi_{m, f e z}^{(0)}=\frac{1}{8 \pi}\left(\phi_{\tilde{c}_{f e z}}^{(0)}+\phi_{c}^{(0)}\right) .
$$

Since we require the other two terms to be zero to leading order this expression insures that each $f e z$ surface intensity will be isotropic.

To find a suitable form for $\left[T_{2}\right]$ we write down the $O(\varepsilon)$ form of our fez closure noting that $\left[T_{3}\right]$ will be zero to $O(\varepsilon)$.

$$
\begin{aligned}
& \psi_{m, f e z}^{(1)}= \begin{cases}{\left[T_{1}\right]_{c \rightarrow \tilde{c}_{f e z}}^{(1)}+\left[T_{2}\right]_{c \rightarrow \tilde{c}_{f e z}}^{(1)},} & \vec{A}_{f e z, w} \cdot \vec{\Omega}>0, \\
{\left[T_{1}\right]_{\tilde{c}_{f e z} \rightarrow c}^{(1)}+\left[T_{2}\right]_{\tilde{c}_{f e z} \rightarrow c}^{(1)},} & \vec{A}_{f e z, w} \cdot \vec{\Omega}<0,\end{cases} \\
&= \begin{cases}\psi_{m, w}^{(1)}+\frac{1}{8 \pi}\left(\phi_{\tilde{c}_{f e z}}^{(1)}-\phi_{c}^{(1)}\right)+\left[T_{2}\right]_{c \rightarrow \tilde{c}_{f f z}}^{(1)}, & \vec{A}_{f e z, w} \cdot \vec{\Omega}>0, \\
\psi_{m, \tilde{w}_{f e z}}^{(1)}+\frac{1}{8 \pi}\left(\phi_{c}^{(1)}-\phi_{\tilde{c}_{f e z}}^{(1)}\right)+\left[T_{2}\right]_{\tilde{c}_{f e z} \rightarrow c}^{(1)}, & \vec{A}_{f e z, w} \cdot \vec{\Omega}<0 .\end{cases}
\end{aligned}
$$

Since we are interested in the $O(\varepsilon)$ net current across the $f e z$ face we multiply this expression by our quadrature direction and sum over all angles. The important quantity is the dot product of this result with $\vec{A}_{f e z, w}$.

$$
\vec{A}_{f e z, w} \cdot \vec{J}_{m, f e z}^{(1)}=\vec{A}_{f e z, w} \cdot \sum_{m} w_{m} \vec{\Omega}_{m} \psi_{m, f e z}^{(1)} .
$$

This expression must be equal to equation (18) if we are to capture the desired diffusion limit. This requirement will guide us in choosing a form for $\left[T_{2}\right]$. To evaluate the $O(1) \mathrm{fez}$ net current we break equation (35) into two half-range sums and replace the surface-average unknown with the expression shown in equation (34).

$$
\begin{aligned}
\vec{A}_{f e z, w} \cdot \vec{J}_{m, f e z}^{(1)}= & \vec{A}_{f e z, w} \cdot \sum_{\vec{A}_{f e z, w} \cdot \bar{\Omega}_{m}>0} w_{m} \vec{\Omega}_{m} \psi_{m, f e z}^{(1)}+\vec{A}_{f e z, w} \cdot \sum_{\bar{A}_{f e z, w} \cdot \vec{\Omega}_{m}<0} w_{m} \vec{\Omega}_{m} \psi_{m, f e z}^{(1)}, \\
= & \vec{A}_{f e z, w} \cdot \sum_{\vec{A}_{f e z, w} \cdot \bar{\Omega}_{m}>0} w_{m} \vec{\Omega}_{m}\left[\psi_{m, w}^{(1)}+\frac{1}{8 \pi}\left(\phi_{\tilde{c}_{f e z}}^{(1)}-\phi_{c}^{(1)}\right)+\left[T_{2}\right]_{c \rightarrow \tilde{c}_{f e z}}^{(1)}\right] \\
& +\vec{A}_{f e z, w} \cdot \sum_{\vec{A}_{f e z, w} \cdot \vec{\Omega}_{m}<0} w_{m} \vec{\Omega}_{m}\left[\psi_{m, \tilde{w}_{f e z}}^{(1)}+\frac{1}{8 \pi}\left(\phi_{c}^{(1)}-\phi_{\tilde{c}_{f e z}}^{(1)}\right)+\left[T_{2}\right]_{\tilde{f}_{f e z} \rightarrow c}^{(1)}\right]
\end{aligned}
$$


Before we can evaluate this summation we must have an expression for the $O(1)$ wedge-average angular flux that is a part of the fez closure as shown in equation (34). We obtain an expression for the wedge-average angular flux from our scaled form of the wedge balance equation.

$$
\begin{gathered}
\psi_{m, w}^{(1)}=\left.\frac{Q_{m}}{\sigma_{t}}\right|_{c} ^{(1)}-\frac{\vec{\Omega}_{m}}{\sigma_{t} V_{w}} \cdot\left[\vec{A}_{f e p, w} \psi_{m, f e p}^{(0)}+\vec{A}_{p e z, w} \psi_{m, p e z}^{(0)}+\vec{A}_{f p z, w} \psi_{m, f p z}^{(0)}+\vec{A}_{f e z, w} \psi_{m, f e z}^{(0)}\right], \\
\psi_{m, \tilde{w}_{f e z}}^{(1)}=\left.\frac{Q_{m}}{\sigma_{t}}\right|_{\tilde{f f e z}_{f e z}} ^{(1)}-\frac{\vec{\Omega}_{m}}{\sigma_{t} V_{w_{f e z}}} \cdot\left[\vec{A}_{f e p^{\prime}, w} \psi_{m, f e p^{\prime}}^{(0)}+\vec{A}_{p^{\prime} e z, w} \psi_{m, p^{\prime} e z}^{(0)}+\vec{A}_{f p^{\prime} z, w} \psi_{m, f p^{\prime} z}^{(0)}-\vec{A}_{f e z, w} \psi_{m, f e z}^{(0)}\right] .
\end{gathered}
$$

We note that the source over sigma terms are once again isotropic

$$
\left.\frac{Q_{m}}{\sigma_{t}}\right|_{c} ^{(1)}=\frac{1}{4 \pi} \phi_{c}^{(1)} \quad \text { and }\left.\quad \frac{Q_{m}}{\sigma_{t}}\right|_{\tilde{c}_{f e z}} ^{(1)}=\frac{1}{4 \pi} \phi_{\tilde{c}_{f e z}}^{(1)} .
$$

The leading order $f e z$ angular flux is isotropic as shown in equation (33). Additionally the fep angular flux is isotropic to leading order in the interior of the problem as shown by equations (25)and (29).

$$
\begin{gathered}
\psi_{m, f e p}^{(0)}=\frac{1}{4 \pi} \phi_{c}^{(0)}=\frac{1}{4 \pi} \phi_{p}^{(0)}, \\
\psi_{m, \text { fep }}^{(0)}=\frac{1}{4 \pi} \phi_{\tilde{c}_{f e z}}^{(0)}=\frac{1}{4 \pi} \phi_{p^{\prime}}^{(0)} .
\end{gathered}
$$

We can simplify equation (37) by inserting the fez leading-order closure and equations (38) and (39).

$$
\begin{aligned}
\psi_{m, w}^{(1)}= & \frac{1}{4 \pi} \phi_{p}^{(1)}-\frac{\bar{\Omega}_{m}}{\sigma_{t} V_{w}} \cdot\left[\vec{A}_{f e p, w} \frac{1}{4 \pi} \phi_{p}^{(0)}+\vec{A}_{f e z, w} \frac{1}{8 \pi}\left(\phi_{p^{\prime}}^{(0)}+\phi_{p}^{(0)}\right)\right] \\
& -\frac{\bar{\Omega}_{m}}{\sigma_{t} l_{w}} \cdot\left[\vec{A}_{p e z, w} \psi_{m, p e z}^{(0)}+\vec{A}_{f p z, w} \psi_{m, f p z}^{(0)}\right], \\
\psi_{m, \tilde{w}_{f e z}}^{(1)} & \frac{1}{4 \pi} \phi_{p^{\prime}}^{(1)}-\frac{\bar{\Omega}_{m}}{\sigma_{t} V_{w f e z}} \cdot\left[\vec{A}_{f e p^{\prime}, w} \frac{1}{4 \pi} \phi_{p^{\prime}}^{(0)}-\vec{A}_{f e z, w} \frac{1}{8 \pi}\left(\phi_{p^{\prime}}^{(0)}+\phi_{p}^{(0)}\right)\right] \\
& -\frac{\bar{\Omega}_{m}}{\sigma_{t} V_{w_{f e z}}} \cdot\left[\vec{A}_{p^{\prime} e z, w} \psi_{m, p^{\prime} e z}^{(0)}+\vec{A}_{f p^{\prime} z, w} \psi_{m, f p^{\prime} z}^{(0)}\right] .
\end{aligned}
$$

Where we used the notation $p$ and $p^{\prime}$ in place of $c$ and $\tilde{c}_{f e z}$ respectively. This notation was established in equation (29) to represent the corner scalar fluxes being equal about a common vertex in the interior of the problem.

Now that we have developed a simplified expression for $\psi_{m, w}^{(1)}$ we return to evaluating equation (36).

We can eliminate the wedge-averaged angular fluxes by using equation (40) to arrive at the following form for the $O(1) \mathrm{fez}$ net current.

$$
\begin{aligned}
& \vec{A}_{f e z, w} \cdot \vec{J}_{m, f e z}^{(1)}=\vec{A}_{f e z, w} \cdot \sum_{\vec{A}_{f e z, w} \cdot \bar{\Omega}_{m}>0} w_{m} \vec{\Omega}_{m}\left[T_{2}\right]_{c \rightarrow \tilde{c}_{f e z}}^{(1)}+\vec{A}_{f e z, w} \cdot \sum_{\bar{A}_{f e z, w} \cdot \bar{\Omega}_{m}<0} w_{m} \vec{\Omega}_{m}\left[T_{2}\right]_{\tilde{c}_{f e z} \rightarrow c}^{(1)} \\
& -\frac{\bar{A}_{f e z, w}}{6 \sigma_{t} V_{w}} \cdot\left(\vec{A}_{f e p, w} \phi_{p}^{(0)}+\vec{A}_{f e p^{\prime}, w} \phi_{p^{\prime}}^{(0)}\right) \\
& -\frac{\bar{A}_{f e z, w}}{\sigma_{t} V_{w}} \cdot\left(\sum_{\bar{A}_{f e z, w} \cdot \bar{\Omega}_{m}>0} w_{m} \vec{\Omega}_{m} \vec{\Omega}_{m} \psi_{m, p e z}^{(0)}\right) \cdot \vec{A}_{p e z, w}-\frac{\bar{A}_{f e z, w}}{\sigma_{t} V_{w}} \cdot\left(\sum_{\bar{A}_{f e z, w} \cdot \bar{\Omega}_{m}<0} w_{m} \vec{\Omega}_{m} \vec{\Omega}_{m} \psi_{m, p^{\prime} e z}^{(0)}\right) \cdot \vec{A}_{p^{\prime} e z, w} \\
& -\frac{\bar{A}_{f e z, w}}{\sigma_{t} V_{w}} \cdot\left(\sum_{\bar{A}_{f e z, w} \cdot \bar{\Omega}_{m}>0} w_{m} \vec{\Omega}_{m} \vec{\Omega}_{m} \psi_{m, f p z}^{(0)}\right) \cdot \vec{A}_{f p z, w}-\frac{\bar{A}_{f f z, w}}{\sigma_{t} V_{w}} \cdot\left(\sum_{\bar{A}_{f e z, w} \cdot w_{m}<0} w_{m} \vec{\Omega}_{m} \vec{\Omega}_{m} \psi_{m, f p^{\prime} z}^{(0)}\right) \cdot \vec{A}_{f p^{\prime} z, w} \cdot
\end{aligned}
$$

Here we have assumed that our quadrature set will integrate the omega-omega tensor correctly.

$$
\sum_{\vec{A}_{f e z, w} \cdot \Omega_{m}>0} w_{m} \vec{\Omega}_{m} \vec{\Omega}_{m}=\sum_{\vec{A}_{f e z, w} \cdot \Omega_{m}<0} w_{m} \vec{\Omega}_{m} \vec{\Omega}_{m}=\frac{2 \pi}{3} \overrightarrow{\vec{I}}
$$


We have also recognized that the referenced wedge volumes are equal, $V_{w}=V_{w_{f e z}}$, if the edge point, $e$, is specified to be the midpoint between the two vertices of interest, $p$ and $p^{\prime}$.

Comparing the $p e z$ and $f p z$ terms against equation (18) we see that our $p e z$ and $f p z$ closures will need to have the following property.

$$
\begin{aligned}
& \sum_{\vec{A}_{f e z, w} \cdot \bar{\Omega}_{m}>0} w_{m} \vec{\Omega}_{m} \vec{\Omega}_{m} \psi_{m, p e z}^{(0)}=\frac{2 \pi}{3} \frac{1}{4 \pi} \frac{1}{3}\left(\phi_{p}^{(0)}+\phi_{e}^{(0)}+\phi_{z}^{(0)}\right), \\
& \sum_{\vec{A}_{f e z, w^{\prime}} \cdot \bar{\Omega}_{m}<0} w_{m} \vec{\Omega}_{m} \vec{\Omega}_{m} \psi_{m, p^{\prime} e z}^{(0)}=\frac{2 \pi}{3} \frac{1}{4 \pi} \frac{1}{3}\left(\phi_{p^{\prime}}^{(0)}+\phi_{e}^{(0)}+\phi_{z}^{(0)}\right), \\
& \sum_{\vec{A}_{f e z, w} \cdot \bar{\Omega}_{m}>0} w_{m} \vec{\Omega}_{m} \vec{\Omega}_{m} \psi_{m, f p z}^{(0)}=\frac{2 \pi}{3} \frac{1}{4 \pi} \frac{1}{3}\left(\phi_{f}^{(0)}+\phi_{p}^{(0)}+\phi_{z}^{(0)}\right), \\
& \sum_{\vec{A}_{f e z,}, w^{\prime},{ }_{\Omega}<0} w_{m} \vec{\Omega}_{m} \vec{\Omega}_{m} \psi_{m, f p^{\prime} z}^{(0)}=\frac{2 \pi}{3} \frac{1}{4 \pi} \frac{1}{3}\left(\phi_{f}^{(0)}+\phi_{p^{\prime}}^{(0)}+\phi_{z}^{(0)}\right) .
\end{aligned}
$$

To ensure that these two closures have this property we have chosen the following expressions.

$$
\begin{gathered}
\psi_{m, p e z}= \begin{cases}\psi_{m, w}+\frac{1}{3} \lambda_{m, w}^{p e z}\left(\left.\frac{Q_{m}}{\sigma_{t}}\right|_{e}+\left.\frac{Q_{m}}{\sigma_{t}}\right|_{z}-\left.2 \frac{Q_{m}}{\sigma_{t}}\right|_{c}\right)+\left[T_{p e z}\right]_{w \rightarrow \tilde{w}_{p e z}}, & \vec{A}_{p e z, w} \cdot \vec{\Omega}_{m}>0, \\
\psi_{m, \tilde{w}_{p e z}}+\frac{1}{3} \lambda_{m, \tilde{w}_{p e z}}^{p e z}\left(\left.\frac{Q_{m}}{\sigma_{t}}\right|_{e}+\left.\frac{Q_{m}}{\sigma_{t}}\right|_{z}-\left.2 \frac{Q_{m}}{\sigma_{t}}\right|_{c}\right)+\left[T_{p e z}\right]_{\tilde{w}_{p e z} \rightarrow w}, & \vec{A}_{p e z, w} \cdot \vec{\Omega}_{m}<0,\end{cases} \\
\psi_{m, f p z}= \begin{cases}\psi_{m, w}+\frac{1}{3} \lambda_{m, w}^{f p z}\left(\left.\frac{Q_{m}}{\sigma_{t}}\right|_{f}+\left.\frac{Q_{m}}{\sigma_{t}}\right|_{z}-\left.2 \frac{Q_{m}}{\sigma_{t}}\right|_{c}\right)+\left[T_{f p z}\right]_{w \rightarrow \tilde{w}_{f p z}}, & \vec{A}_{f p z, w} \cdot \vec{\Omega}_{m}>0, \\
\psi_{m, \tilde{w}_{f p z}}+\frac{1}{3} \lambda_{m, \tilde{w}_{f p z}}^{f p z}\left(\left.\frac{Q_{m}}{\sigma_{t}}\right|_{f}+\left.\frac{Q_{m}}{\sigma_{t}}\right|_{z}-\left.2 \frac{Q_{m}}{\sigma_{t}}\right|_{c}\right)+\left[T_{f p z}\right]_{\tilde{w}_{f p z} \rightarrow w}, & \vec{A}_{f p z, w} \cdot \vec{\Omega}_{m}<0 .\end{cases}
\end{gathered}
$$

We have defined $\tilde{w}_{p e z}$ and $\tilde{w}_{f p z}$ as before. These indexes refer to the wedge that shares the $p e z$ or $f p z$ face with wedge $w$, respectively. Note that we will require the undefined term to be a higher-order term that will allow us to improve our method's performance in other limits without impacting its ability to capture a diffusion solution in the thick limit. We will require this term to be zero to leading order. We will examine this term in a later section. Additionally, we have included the term $\lambda_{m, w}^{x x x}$ that must approach unity as the problem becomes thick and diffusive. We choose the following form for $\lambda_{m, w}^{x x x}$ so that our closures will have the needed form for our scheme to limit Palmer's diffusion discretization. For optically thin problems $\lambda_{m, w}^{x x}$ approaches zero so that the middle term of these closures does not impact the solution in a regime that they was not designed for.

$$
\lambda_{m, w}^{x x x}=\frac{\left(\sigma_{t} V_{w}\right)^{2}}{\left(\sigma_{t} V_{w}\right)^{2}+\left(\vec{A}_{x x, w} \cdot \vec{\Omega}_{m}\right)^{2}}
$$

where $x x x \in\{p e z, f p z, f e z\}$. 
If the $p e z$ and $f p z$ closures have this form then the $O(1)$ current across the $f e z$ face will have the following form (remember that $\lambda_{m, w}^{x x x} \rightarrow 1$ in the asymptotic limit).

$$
\begin{aligned}
& \vec{A}_{f e z, w} \cdot \vec{J}_{m, f e z}^{(1)}= \vec{A}_{f e z, w} \cdot \sum_{\vec{A}_{f e z, w} \cdot \vec{\Omega}_{m}>0} w_{m} \vec{\Omega}_{m}\left[T_{2}\right]_{c \rightarrow \tilde{c}_{f e z}}^{(1)}+\vec{A}_{f e z, w} \cdot \sum_{\vec{A}_{f e z, w} \cdot \vec{\Omega}_{m}<0} w_{m} \vec{\Omega}_{m}\left[T_{2}\right]_{\tilde{c}_{f e z} \rightarrow c}^{(1)}{ }^{(0)} \\
&-\frac{1}{6 \sigma_{t} V_{w}} \vec{A}_{f e z, w} \cdot\left[\begin{array}{l}
\left(\vec{A}_{f e p, w} \phi_{p}^{(0)}+\vec{A}_{f e p^{\prime}, w} \phi_{p^{\prime}}^{(0)}\right) \\
+\frac{1}{3} \vec{A}_{p e z, w}\left(\phi_{p}^{(0)}+\phi_{e}^{(0)}+\phi_{z}^{(0)}\right)+\frac{1}{3} \vec{A}_{p^{\prime} e z, w}\left(\phi_{p^{\prime}}^{(0)}+\phi_{e}^{(0)}+\phi_{z}^{(0)}\right) \\
+\frac{1}{3} \vec{A}_{f p z, w}\left(\phi_{f}^{(0)}+\phi_{p}^{(0)}+\phi_{z}^{(0)}\right)+\frac{1}{3} \vec{A}_{f p^{\prime} z, w}\left(\phi_{f}^{(0)}+\phi_{p^{\prime}}^{(0)}+\phi_{z}^{(0)}\right)
\end{array}\right] .
\end{aligned}
$$

Comparing the fep terms against equation (18) reveals the following requirement for $\left[T_{2}\right]$.

$$
\begin{aligned}
& \vec{A}_{f e z, w} \cdot \sum_{\vec{A}_{f e z, w} \cdot \bar{\Omega}_{m}>0} w_{m} \vec{\Omega}_{m}\left[T_{2}\right]_{c \rightarrow \tilde{c}_{f e z}}^{(1)}=\frac{-1}{6 \sigma_{t} V_{w}} \vec{A}_{f e z, w} \cdot\left[\frac{1}{3} \vec{A}_{f e p, w}\left(\phi_{f}^{(0)}+\phi_{e}^{(0)}-2 \phi_{p}^{(0)}\right)\right], \\
& \vec{A}_{f e z, w} \cdot \sum_{\vec{A}_{f e z, w} \cdot \bar{\Omega}_{m}<0} w_{m} \vec{\Omega}_{m}\left[T_{2}\right]_{\tilde{c}_{f e z} \rightarrow c}^{(1)}=\frac{-1}{6 \sigma_{t} V_{w}} \vec{A}_{f e z, w} \cdot\left[\frac{1}{3} \vec{A}_{f e p^{\prime}, w}\left(\phi_{f}^{(0)}+\phi_{e}^{(0)}-2 \phi_{p^{\prime}}^{(0)}\right)\right] .
\end{aligned}
$$

There are many forms for $\left[T_{2}\right]$ that could yield this result. To keep things simple, we have chosen to use the following expression that is similar to our $p e z$ and $f p z$ closures.

$$
\begin{aligned}
& {\left[T_{2}\right]_{c \rightarrow \tilde{c}_{f e z}}=\frac{-1}{3 \sigma_{t} t_{w}} \lambda_{m, w}^{f e p} \vec{A}_{f e p, w} \cdot \vec{\Omega}_{m}\left(\left.\frac{Q_{m}}{\sigma_{t}}\right|_{f}+\left.\frac{Q_{m}}{\sigma_{t}}\right|_{e}-\left.2 \frac{Q_{m}}{\sigma_{t}}\right|_{c}\right),} \\
& {\left[T_{2}\right]_{\tilde{c}_{f e z} \rightarrow c}=\frac{-1}{3 \sigma_{t} v_{\tilde{w} f e z}} \lambda_{m, \tilde{w}_{f e z}}^{f e p} \vec{A}_{f e p^{\prime}, w} \cdot \vec{\Omega}_{m}\left(\left.\frac{Q_{m}}{\sigma_{t}}\right|_{f}+\left.\frac{Q_{m}}{\sigma_{t}}\right|_{e}-\left.2 \frac{Q_{m}}{\sigma_{t}}\right|_{\tilde{c}_{f e z}}\right) .}
\end{aligned}
$$

Note that $\left[T_{2}\right]$ will be zero to leading order and to $O(1)$ it reduces to equation (47) as we have required.

Once again we have included the function $\lambda_{m, w}^{x x}$ defined in equation (45) that turns off this difference of sources in thin problems where its impact not important.

Using equations (30), (31) and (48), the fez closure will have the following form.

$$
\psi_{m, f e z}= \begin{cases}\psi_{m, w}+\frac{1}{2}\left(\left.\frac{Q_{m}}{\sigma_{t}}\right|_{\tilde{c}_{f e z}}-\left.\frac{Q_{m}}{\sigma_{t}}\right|_{c}\right) \\ -\frac{1}{3 \sigma_{t} V_{w}} \lambda_{m, w}^{f e z} \vec{A}_{f e p, w} \cdot \vec{\Omega}_{m}\left(\left.\frac{Q_{m}}{\sigma_{t}}\right|_{f}+\left.\frac{Q_{m}}{\sigma_{t}}\right|_{e}-\left.2 \frac{Q_{m}}{\sigma_{t}}\right|_{c}\right)+\left[T_{3}\right]_{c \rightarrow \tilde{c}_{f f z}}, & \vec{A}_{f e z, w} \cdot \vec{\Omega}>0, \\ \psi_{m, \tilde{w}_{f e z}}+\frac{1}{2}\left(\left.\frac{Q_{m}}{\sigma_{t}}\right|_{c}-\left.\frac{Q_{m}}{\sigma_{t}}\right|_{\tilde{c}_{f f e z}}\right) \\ -\frac{1}{3 \sigma_{t} t_{\tilde{w} f e z}} \lambda_{m, \tilde{w}_{f e z}}^{f e z} \vec{A}_{f e p^{\prime}, w} \cdot \vec{\Omega}_{m}\left(\left.\frac{Q_{m}}{\sigma_{t}}\right|_{f}+\left.\frac{Q_{m}}{\sigma_{t}}\right|_{e}-\left.2 \frac{Q_{m}}{\sigma_{t}}\right|_{\tilde{c}_{f e z}}\right)+\left[T_{3}\right]_{\tilde{c}_{f e z} \rightarrow c}, & \vec{A}_{f e z, w} \cdot \vec{\Omega}<0 .\end{cases}
$$

Since $\left[T_{3}\right]$ is zero in the diffusion limit we are assured of capturing the desired diffusion solution in this limit. The closure requirements outlined in equations (24), (44) and (49) are needed to achieve this goal. In practice we replace $\psi_{m, w}$ using the wedge balance equation (6). This leaves us with the follow fez closure. 


$$
\begin{aligned}
& \psi_{m, f e z}\left(1+\frac{\left|\vec{\Omega}_{m} \cdot \bar{A}_{f e z, w}\right|}{\sigma_{t} V_{w}}\right) \\
& \left(\frac{1}{2}\left(\left.\frac{Q_{m}}{\sigma_{t}}\right|_{\tilde{c}_{f e z}}+\left.\frac{Q_{m}}{\sigma_{t}}\right|_{c}\right)-\lambda_{m, w}^{f e z} \frac{\bar{A}_{f e p, w} \cdot \vec{\Omega}_{m}}{3 \sigma_{t} V_{w}}\left(\left.\frac{Q_{m}}{\sigma_{t}}\right|_{f}+\left.\frac{Q_{m}}{\sigma_{t}}\right|_{e}-\left.2 \frac{Q_{m}}{\sigma_{t}}\right|_{c}\right)+\left[T_{3}\right]_{c \rightarrow \tilde{c}_{f e z}}\right. \\
& = \begin{cases}-\frac{\vec{\Omega}_{m}}{\sigma_{t} V_{w}} \cdot\left(\vec{A}_{f e p, w} \psi_{m, f e p}+\vec{A}_{p e z, w} \psi_{m, p e z}+\vec{A}_{f p z, w} \psi_{m, f p z}\right), & \vec{A}_{f e z, w} \cdot \vec{\Omega}>0,\end{cases} \\
& \mid \frac{1}{2}\left(\left.\frac{Q_{m}}{\sigma_{t}}\right|_{c}+\left.\frac{Q_{m}}{\sigma_{t}}\right|_{\tilde{c}_{f e z}}\right)-\lambda_{m, \tilde{w}_{f e z}}^{f e z} \frac{\bar{A}_{f e p^{\prime}, w^{\prime}} \cdot \bar{\Omega}_{m}}{3 \sigma_{t} \tilde{w}_{\tilde{w} f e z}}\left(\left.\frac{Q_{m}}{\sigma_{t}}\right|_{f}+\left.\frac{Q_{m}}{\sigma_{t}}\right|_{e}-\left.2 \frac{Q_{m}}{\sigma_{t}}\right|_{\tilde{c}_{f e z}}\right)+\left[T_{3}\right]_{\tilde{\tau}_{f e z} \rightarrow c} \\
& -\frac{\vec{\Omega}_{m}}{\sigma_{t} V_{w}} \cdot\left(\vec{A}_{f e p^{\prime}, w} \psi_{m, f e p^{\prime}}+\vec{A}_{p^{\prime} e z, w} \psi_{m, p^{\prime} e z}+\vec{A}_{f p^{\prime} z, w} \psi_{m, f p^{\prime} z}\right), \quad \vec{A}_{f e z, w} \cdot \vec{\Omega}<0 .
\end{aligned}
$$

We have deferred the development of $\left[T_{3}\right]$ to the next section since it does not impact the solution our method obtains in the diffusion limit.

\section{F. Leading-Order Boundary Condition Implications}

The remaining unanswered question about the behavior of 3D UCB in thick diffusive problems is the boundary condition satisfied by the leading-order solution. To examine this question we rewrite equation (28) assuming that $\vec{A}_{f e p, w}$ is on the problem boundary.

$$
\vec{A}_{f e p, w} \cdot\left[\sum_{\bar{A}_{f e p, w} \cdot \bar{\Omega}_{m}>0} w_{m} \vec{\Omega}_{m} \frac{1}{4 \pi} \phi_{c}^{(0)}+\sum_{\bar{A}_{f e p, w} \cdot \bar{\Omega}_{m}<0} w_{m} \vec{\Omega}_{m} \psi_{m, \text { fep }}^{i n c,(0)}\right]=0, \quad \vec{r} \in \Gamma .
$$

Note that we have used the $f e p$ closure defined in equation (24) to arrive at this form.

We are looking for an expression for $\phi_{\tilde{c}_{\text {fep }}}^{(0)}$ on the boundary. Comparing equation (51) and equation (28) we see that on the boundary of the problem we can define an expression for the leading-order scalar flux so that equation (28) holds even when we are on the boundary.

$$
\phi_{\tilde{c}_{f e p}^{(0)}}^{(0)}=\frac{-4 \pi \sum_{\vec{f}_{f e p, w} \cdot \bar{\Omega}_{m}<0} w_{m}\left(\vec{A}_{f e p, w} \cdot \vec{\Omega}_{m}\right) \psi_{f e p, w}^{i n c,(0)}}{\sum_{\bar{A}_{f e p, w} \cdot \bar{\Omega}_{m}>0} w_{m}\left(\vec{A}_{f e p, w} \cdot \vec{\Omega}_{m}\right)}, \quad \vec{r} \in \Gamma .
$$

We can simplify this expression by defining two parameters, $\vec{n}$ and $\rho$.

$$
\begin{aligned}
& \vec{n}=\frac{\vec{A}_{f e p, w}}{\left|\vec{A}_{f e p, w}\right|}, \\
& \rho=\frac{1}{\pi} \sum_{\vec{n} \cdot \mathbf{\Omega}>0} w_{m}(\vec{n} \cdot \vec{\Omega})=\frac{1}{\pi} \sum_{\vec{n} \cdot \vec{\Omega}<0} w_{m}|\vec{n} \cdot \vec{\Omega}| \approx 1,
\end{aligned}
$$

which leaves us with an expression for $\phi_{\tilde{c}_{\text {fep }}}^{(0)}$ on the boundary of the problem

$$
\phi_{\tilde{c}_{\text {fep }}}^{(0)}=\frac{2}{\rho} \sum_{\vec{n} \cdot \bar{\Omega}_{m}<0} w_{m} 2\left|\vec{n} \cdot \vec{\Omega}_{m}\right| \psi_{m, f e p}^{i n c,(0)}, \quad \vec{r} \in \Gamma .
$$

This is a discrete Marshak boundary condition, which can cause both the boundary and interior solutions to be inaccurate. When compared to equation (12) we see that this inaccuracy may be significant 
if the incident flux is grazing such that $\vec{n} \cdot \vec{\Omega}<<1$. In $2 \mathrm{D}$ Adams ${ }^{11}$ has had some success in improving this limit by modifying a closure equation that takes on the same role as our $f e z$ closure. Unfortunately, the adjustments made by Adams are not tractable for our 3D wedge-based method.

\section{G. Closure Development - Improving Performance for Source-Free Purely Absorbing Media}

The system we have defined so far has three terms that remain undefined. Each of these terms was originally included into our system to allow us to improve the behavior of our method outside of the optically thick, highly scattering limit. We want to make sure our scheme has good performance characteristics in problems where there is no scattering and no distributed sources (including fission). In such a source-free purely absorbing medium our method should produce a purely positive and monotonic solution. In this section we are interested in completing the definitions of our closure equations so that we more closely approximate the analytic exponential form of the solution for optically thin cells.

The UCB method has had a great deal of success in 2D with an extrapolation term in the fez closure. Extending this form (Adams, 1997) to 3D we start with the following for $\left[T_{f e z}\right]=\left[T_{3}\right]_{c \rightarrow \tilde{c}_{f a}}$.

$$
\left[T_{f e z}\right]=\left[\frac{3+4 \tau}{2 \tau\left(1+\tau+2 \tau^{2}\right)}\right]_{m, f e z} \cdot\left[\psi_{m, w}-\psi_{m, w}^{i n c}\right] .
$$

The term $\tau_{m, f e z}$ represents an optical thickness of the wedge in which we approximate the width of the wedge as the ratio of the wedge volume to the $f e z$ face area. $\psi_{m, w}^{i n c}$ represents the incident angular flux along the current discrete ordinate direction weighted by the area projection of each face along this direction.

$$
\begin{aligned}
& \tau_{m, x x x}=\frac{\sigma_{t} V_{w}}{\left|\vec{A}_{x x x, w} \cdot \vec{\Omega}_{m}\right|}, \\
& \psi_{m, w}^{i n c}=\frac{\sum_{x x x: \bar{x}_{x x, w} \cdot \bar{\Omega}_{m}<0} \vec{A}_{x x x, w} \cdot \vec{\Omega}_{m} \psi_{x x x, m, w}^{i n c}}{\sum_{x x x: \vec{A}_{x x x, w} \cdot \vec{\Omega}_{m}<0} \vec{A}_{x x x, w} \cdot \vec{\Omega}_{m}} . \quad \text { where } x x x \in\{f e z, f e p, p e z, f p z\}
\end{aligned}
$$

With the fez closure complete we concentrate on finishing the fep and fep closures. We will choose these two terms to maximize our performance in a source free, purely absorbing, slab-like, orthogonal grid problem. We examine the line-of-sight path through 2 corners (or 6 wedges) of a zone as shown below in Figure 5. This approach is slab-like because we only consider a line-of-sight path in the $x$-direction. We assume that this path never crosses the top, bottom, left or right faces of the corners shown in Figure 5. 

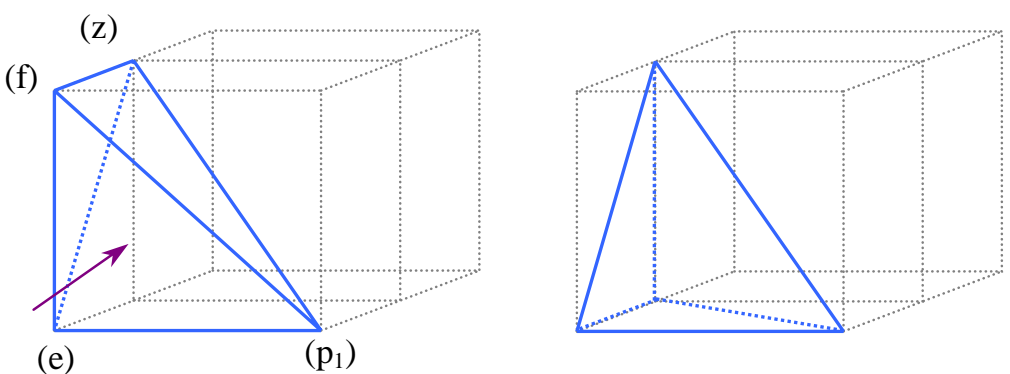

(e)
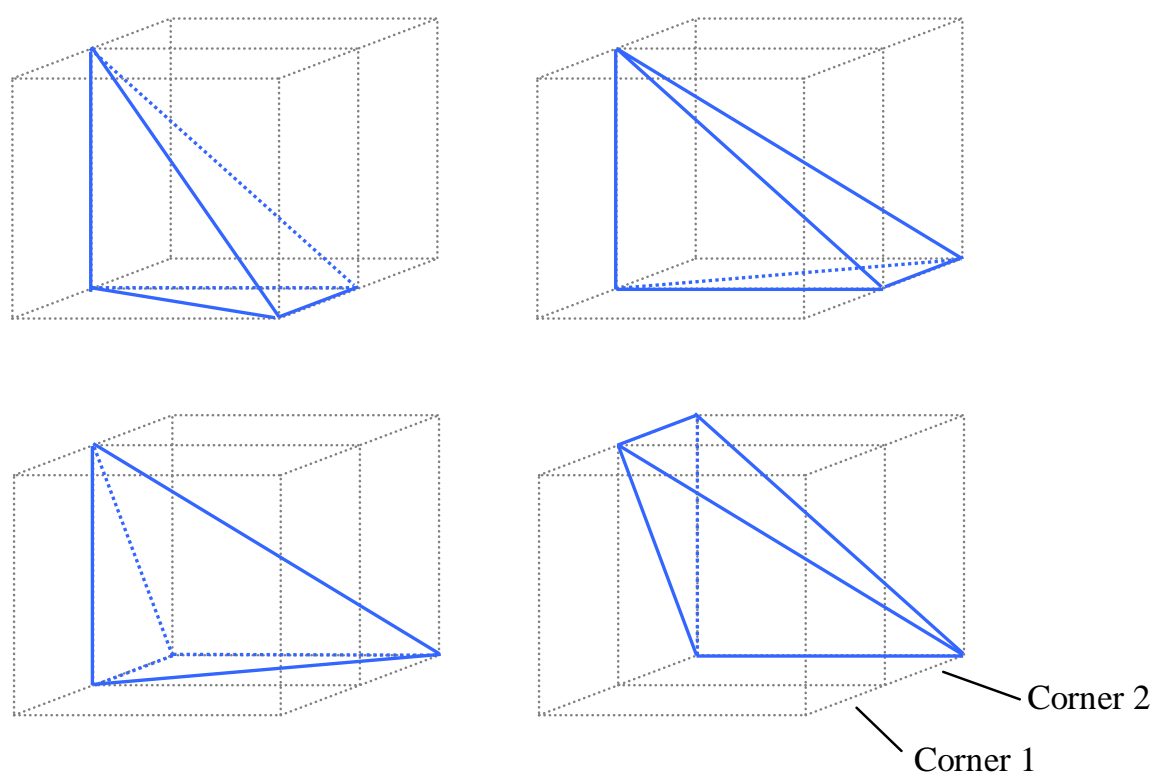

Figure 5. Sweeping through part of a zone.

The simplified balance and closure equations for this scenario are listed here.

$$
\begin{array}{rlr}
\left(\vec{A}_{f e z, w} \cdot \vec{\Omega}_{m}\right) \psi_{f e z, m, w}+\left(\vec{A}_{f e p, w} \cdot \vec{\Omega}_{m}\right) \psi_{f e p, m, w}+\left(\vec{A}_{f p z, w} \cdot \vec{\Omega}_{m}\right) \psi_{f p z, m, w} \\
\quad+\left(\vec{A}_{p e z, w} \cdot \vec{\Omega}_{m}\right) \psi_{p e z, m, w}+\sigma_{t} V_{w} \psi_{w, m}=0, & \\
\psi_{f e p, m, w}=\psi_{m, w}, & \vec{A}_{f e p, w} \cdot \vec{\Omega}_{m}>0, \\
\psi_{f e z, m, w} & =\psi_{m, w}+\frac{3+\tau_{m, f e z}}{2 \tau_{m, f e z}\left(1+\tau_{m, f e z}+2 \tau_{m, f e z}^{2}\right)}\left(\psi_{m, w}-\psi_{m, w}^{i n c}\right), & \vec{A}_{f e z, w} \cdot \vec{\Omega}_{m}>0, \\
\psi_{p e z, m, w}=\psi_{m, w}+\beta\left(\tau_{m, p e z}\right) \cdot\left(\psi_{m, w}-\psi_{m, w}^{i n c}\right), & \vec{A}_{p e z, w} \cdot \vec{\Omega}_{m}>0, \\
\psi_{f p z, m, w}=\psi_{m, w}+\beta\left(\tau_{m, f p z}\right) \cdot\left(\psi_{m, w}-\psi_{m, w}^{i n c}\right), & \vec{A}_{f p z, w} \cdot \vec{\Omega}_{m}>0 .
\end{array}
$$

We have assumed that $\left[T_{f p z}\right]$ and $\left[T_{p e z}\right]$ have a form similar to $\left[T_{f e z}\right]$ but we have not fully defined the parameters $\beta\left(\tau_{m, p e z}\right)$ and $\beta\left(\tau_{m, f p z}\right)$. We will choose $\beta$ so that our scheme will capture some approximation of the source-free, purely absorbing solution. Since the analytic solution will be an exponential in $\tau_{m, x x x}$ we would like our scheme to capture a Pade' approximation of this exponential. 
Using the equations in (57) we can show that the attenuation factor for the zone (passing through the six wedges shown in Figure 5) will be

$$
f\left(\tau_{m, x x x}\right)=\frac{\left[\begin{array}{c}
\left(\tau_{m, p e z} \beta\left(\tau_{m, p e z}\right)-\beta\left(\tau_{m, p e z}\right)-1\right)^{2} \cdot\left(\tau_{m, f p z} \beta\left(\tau_{m, f p z}\right)-\beta\left(\tau_{m, f p z}\right)-1\right)^{2} \\
\cdot\left(\tau_{m, f e z} \gamma\left(\tau_{m, f e z}\right)-\gamma\left(\tau_{m, f e z}\right)-1\right)
\end{array}\right]}{\left(\tau_{m, f e p}+1\right)\left(\beta\left(\tau_{m, p e z}\right)+\tau_{m, p e z}+1\right)^{2} \cdot\left(\beta\left(\tau_{m, f p z}\right)+\tau_{m, f p z}+1\right)^{2} \cdot\left(\gamma\left(\tau_{m, f e z}\right)+\tau_{m, f e z}+1\right)},
$$

where

$$
\gamma\left(\tau_{m, f e z}\right)=\frac{3+4 \tau_{m, f e z}}{2 \tau_{m, f e z}\left(1+\tau_{m, f e z}+2 \tau_{m, f e z}^{2}\right)} .
$$

Note that we have assumed that for non-zero values the $\vec{A} \cdot \vec{\Omega}$ terms will all have the same magnitude, but not necessarily the same sign. That is, we assume for this development that the corner faces are parallel and of equal area. We also note that based on our definition of the optical thickness of a wedge, the optical thickness of the zone for our direction of interest will be $\left(2 \tau_{m, p e z}+2 \tau_{m, f p z}+\tau_{m, f e z}+\tau_{m, f e p}\right)$. This thickness assumes that we pass through the zone ( 2 corners) along a path that does not traverse the top, bottom, left or right corner boundaries. The analytic form for the attenuation factor for this path will be $f_{\text {analytic }}=e^{-2 \tau_{m, p e z}} e^{-2 \tau_{m, f p z}} e^{-\tau_{m, f e z}} e^{-\tau_{m, f e p}}$.

Since we would like both $\beta$ and $f\left(\tau_{m, x x x}\right)$ to be positive, monotonic and smooth we will assume a polynomial form for $\beta$.

$$
\beta\left(\tau_{m, x x x}\right)=\frac{1}{a+b \tau_{m, x x x}+c \tau_{m, x x x}^{2}} .
$$

We may determine appropriate values for $a, b$ and $c$ by substituting our form for $\beta$ into $f\left(\tau_{m, x x x}\right)$, expanding in a Taylor series about $\tau_{m, x x x}=0$, and comparing this to the Taylor series expansion for the analytic solution. For the special case that we are examining we also note that $\tau_{m, w}=\tau_{m, p e z}=\tau_{m, f p z}=\tau_{m, f e z}=\tau_{m, f e p}$, so that the analytic and our single cell attenuation factors have the following expansions.

$$
\begin{aligned}
e^{-6 \tau} & =1-6 \tau_{m, w}+18 \tau_{m, w}^{2}-36 \tau_{m, w}^{3}+54 \tau_{m, w}^{4}+O\left(\tau_{m, w}^{5}\right), \\
f & =1-6 \tau_{m, w}+4 \frac{5 a+4}{a+1} \tau_{m, w}^{2}-\frac{4}{3} \frac{37 a^{2}+53 a-3 b+19}{(a+1)^{2}} \tau_{m, w}^{3} \\
& +\frac{101 a^{3}+195 a^{2}+125 a-28 a b+4 a c+27-4 b^{2}-20 b+4 c}{(a+1)^{3}} \tau_{m, w}^{4}+O\left(\tau_{m, w}^{5}\right) .
\end{aligned}
$$

By comparing these expansions term by term we specify the fully defined form for $\beta$.

$$
\beta\left(\tau_{m, x x x}\right)=\frac{1}{1+\frac{1}{3} \tau_{m, x x x}+\frac{1}{18} \tau_{m, x x x}^{2}} .
$$

This expression will captures the analytic solution to $O\left(\tau^{4}\right)$ for slab-like problems. 


\section{H. Source Extrapolations}

The only terms that have not been defined are the $Q_{m} / \sigma_{t}$ terms evaluated at each vertex of a wedge. Typically, we are only given volumetric-averaged source values for each corner. We have chosen to define the remaining source terms by using a zone-averaged gradient extrapolation similar to the one Palmer uses to define the scalar flux. The zone centered source term is defined as a function of the point- and facecenter fluxes.

$$
\begin{aligned}
& \left.\frac{Q_{m}}{\sigma_{t}}\right|_{z}=\sum_{w \in z} \omega_{w}\left(\left.\frac{Q_{m}}{\sigma_{t}}\right|_{c}+\vec{l}_{p z, w} \cdot\left\langle\vec{\nabla} \frac{Q_{m}}{\sigma_{t}}\right\rangle_{z}\right), \\
& \omega_{w}=\frac{\frac{1}{\left|\vec{l}_{p z, w}\right|}}{\sum_{w \in z} \frac{1}{\left|\vec{l}_{p z, w}\right|}}, \quad\left\langle\vec{\nabla} \frac{Q_{m}}{\sigma_{t}}\right\rangle_{z}=\frac{1}{3 V_{z}} \sum_{w \in z} \vec{A}_{f e p}\left(\left.\frac{Q_{m}}{\sigma_{t}}\right|_{f}+\left.\frac{Q_{m}}{\sigma_{t}}\right|_{e}+\left.\frac{Q_{m}}{\sigma_{t}}\right|_{c}\right) .
\end{aligned}
$$

The corner, $c$, and zone, $z$, terms represent the corner or zone that contain wedge $w$. The notation $w \in z$ represents all the wedges contained in the current zone. The vector $\vec{l}_{p z, w}$ points from the vertex $(p)$ to the zone center $(z)$ for the wedge. This expression also includes source over sigma terms for the zone faces $(f)$ and edge points $(e)$. These terms are defined next.

The face-centered source term is defined in a similar way.

$$
\begin{aligned}
& \left.\frac{Q_{m}}{\sigma_{t}}\right|_{f}=\sum_{w \in f} v_{w}\left(\left.\frac{Q_{m}}{\sigma_{t}}\right|_{c}+\vec{l}_{p f, w} \cdot\left\langle\vec{\nabla} \frac{Q_{m}}{\sigma_{t}}\right\rangle_{f}\right), \\
& v_{w}=\frac{1}{\sum_{w \in f} \frac{1}{\left|\vec{l}_{p f, w}\right|}}, \quad\left\langle\vec{\nabla} \frac{Q_{m}}{\sigma_{t}}\right\rangle_{f}=\left.\frac{1}{\left|\vec{A}_{f}\right|} \sum_{w \in f}\left(\vec{l}_{p e, w} \times \frac{\bar{A}_{f e p}}{\left|\vec{A}_{f e p}\right|}\right) \cdot \frac{Q_{m}}{\sigma_{t}}\right|_{c} .
\end{aligned}
$$

The cross product in the face-averaged gradient term represents a vector with length $|\overrightarrow{p e}|$ that points away from the wedge and is perpendicular to both $\overrightarrow{p e}$ and $\vec{A}_{f e p}$ (see Figure 4). As before $\vec{l}_{p f, w}$ is the vector from the zone vertex $(p)$ to the face center $(f)$ for the current wedge. $\left|\vec{A}_{f}\right|$ is the projected surface area of the entire zone face that contains point $(f)$ (i.e. $\vec{A}_{f}=\sum_{w \in f} \vec{A}_{f e p}$ ).

The edge centered source term is a simple average of the two vertex source terms it separates. It is a simple average because we have defined the edge point to always be the midpoint of the line that connects these two vertices. We would like to remind the reader that we have assumed that the cross section, $\sigma_{t}$, is equal for all corners in the cell.

$$
\left.\frac{Q_{m}}{\sigma_{t}}\right|_{e}=\frac{1}{2}\left(\left.\frac{Q_{m}}{\sigma_{t}}\right|_{c}+\left.\frac{Q_{m}}{\sigma_{t}}\right|_{\tilde{c}_{f e z}}\right)
$$

With these definitions we have fully defined our spatial scheme. 


\section{I. Closure Development - Summary}

We have completed a fairly detailed derivation of the balance equations and closure equations that define the 3D UCBL spatial discretization scheme. We summarize these results here and briefly discuss how this scheme in implemented in a transport code.

We have a pseudo-balance equation on each tetrahedral wedge.

$$
\begin{aligned}
& \vec{A}_{f e z, w} \cdot \vec{\Omega}_{m} \psi_{m, f e z}+\vec{A}_{f e p, w} \cdot \vec{\Omega}_{m} \psi_{m, f e p}+\vec{A}_{p e z, w} \cdot \vec{\Omega}_{m} \psi_{m, p e z} \\
& \quad+\vec{A}_{f p z, w} \cdot \vec{\Omega}_{m} \psi_{m, f p z}+V_{w} \sigma_{t} \psi_{m, w}=V_{w} Q_{m, c} .
\end{aligned}
$$

The area vectors are outward normals for each of the tetrahedral faces. We use the nomenclature fez, fep, $p e z$ or $f p z$ to designate triangular faces defined by three of the vertices (face, edge, zone, point) that define the wedge. It may be useful to refer to Figures 1 and 2 where these points are labeled on a picture of a wedge. The surface-averaged angular flux uses this same nomenclature. The volume-averaged angular flux is designated by $\psi_{m, w}$, the volume of the wedge by $V_{w}$, the quadrature direction by $\vec{\Omega}_{m}$, the total loss cross section by $\sigma_{t}$ and the total corner-averaged source by $Q_{m, c}$.

We should note that this equation can be summed over all wedges in a corner to produce a true balance equation for a corner volume. In the same way we can sum all of the wedges in a cell to produce a balance equation for each cell. This scheme is a subcell balance method because it preserves balance on both the cell and the corner (subcell). It does not preserve balance on wedges.

To complete our system we derived closure equations that relate each of the wedge surface-average angular fluxes to wedge volume-averaged quantities. The $f e p$ closure is the traditional step closure on the surface of the cell. This closure is common to all corner balance schemes developed to date.

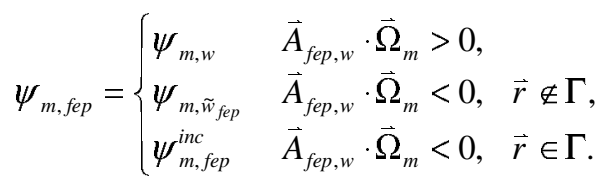

The $p e z$ and $f p z$ closures are new to 3D UCBL. These closures are for corner-interior surfaces. They were derived to limit to the desired diffusion discretization in the thick diffusion limit (first two terms) and to reduce truncation error in thin slab-like problems (last term).

$$
\begin{gathered}
\psi_{m, p e z}= \begin{cases}\psi_{m, w}+\frac{1}{3} \lambda_{m, w}^{p e z}\left(\left.\frac{Q_{m}}{\sigma_{t}}\right|_{e}+\left.\frac{Q_{m}}{\sigma_{t}}\right|_{z}-\left.2 \frac{Q_{m}}{\sigma_{t}}\right|_{c}\right)+\beta\left(\tau_{m, p e z}\right)\left[\psi_{m, w}-\psi_{m, w}^{i n c}\right], & \vec{A}_{p e z, w} \cdot \vec{\Omega}_{m}>0, \\
\psi_{m, \tilde{w}_{p e z}}+\frac{1}{3} \lambda_{m, \tilde{w}_{p e z}}^{p e z}\left(\left.\frac{Q_{m}}{\sigma_{t}}\right|_{e}+\left.\frac{Q_{m}}{\sigma_{t}}\right|_{z}-\left.2 \frac{Q_{m}}{\sigma_{t}}\right|_{c}\right)+\beta\left(\tau_{m, \tilde{w}_{p e z}}\right)\left[\psi_{m, \tilde{w}_{p e z}}-\psi_{m, \tilde{w}_{p e z}}^{i n c}\right], & \vec{A}_{p e z, w} \cdot \vec{\Omega}_{m}<0,\end{cases} \\
\psi_{m, f p z z}= \begin{cases}\psi_{m, w}+\frac{1}{3} \lambda_{m, w}^{f p z}\left(\left.\frac{Q_{m}}{\sigma_{t}}\right|_{f}+\left.\frac{Q_{m}}{\sigma_{t}}\right|_{z}-\left.2 \frac{Q_{m}}{\sigma_{t}}\right|_{c}\right)+\beta\left(\tau_{m, f p z}\right)\left[\psi_{m, w}-\psi_{m, w}^{i n c}\right], & \vec{A}_{f p z, w} \cdot \vec{\Omega}_{m}>0, \\
\psi_{m, \tilde{w} f p z}+\frac{1}{3} \lambda_{m, \tilde{w}_{f p z}}^{f z z}\left(\left.\frac{Q_{m}}{\sigma_{t}}\right|_{f}+\left.\frac{Q_{m}}{\sigma_{t}}\right|_{z}-\left.2 \frac{Q_{m}}{\sigma_{t}}\right|_{c}\right)+\beta\left(\tau_{m, \tilde{w}_{f p z}}\right)\left[\psi_{m, \tilde{w}_{f p z}}-\psi_{m, \tilde{w}_{f p z}}^{i n c}\right], & \vec{A}_{f p z, w} \cdot \vec{\Omega}_{m}<0 .\end{cases}
\end{gathered}
$$


The $\beta$ terms used in this closure are defined by equation (62) and the $\lambda_{m, w}^{x x x}$ terms are defined by equation (45). The source over sigma terms are defined in equations (63) - (65) and the projection-areaweighted incident angular flux term is defined in equation (56).

The $f e z$ closure relates within-cell corner surface quantities to volume-averaged quantities. It was derived as an extension of the analogous closure in 2D UCB. The terms were modified slightly to obtain the desired form of the net current density in the thick diffusion limit.

$$
\begin{aligned}
& \psi_{m, f e z}\left(1+\frac{\left|\vec{\Omega}_{m} \cdot \vec{A}_{f e z, w}\right|}{\sigma_{t} V_{w}}\right) \\
& \left(\frac{1}{2}\left(\left.\frac{Q_{m}}{\sigma_{t}}\right|_{\tilde{c}_{f e z}}+\left.\frac{Q_{m}}{\sigma_{t}}\right|_{c}\right)-\lambda_{m, w}^{f e z} \frac{\bar{A}_{f e p, w} \cdot \bar{\Omega}_{m}}{3 \sigma_{t} V_{w}}\left(\left.\frac{Q_{m}}{\sigma_{t}}\right|_{f}+\left.\frac{Q_{m}}{\sigma_{t}}\right|_{e}-\left.2 \frac{Q_{m}}{\sigma_{t}}\right|_{c}\right)\right. \\
& =\left\{\begin{array}{l}
+\gamma\left(\tau_{m, f e z}\right)\left[\psi_{m, w}-\psi_{m, w}^{i n c}\right]-\frac{\bar{\Omega}_{m}}{\sigma_{t} V_{w}} \cdot\left(\vec{A}_{f e p, w} \psi_{m, f e p}+\vec{A}_{p e z, w} \psi_{m, p e z}\right. \\
\frac{1}{2}\left(\left.\frac{Q_{m}}{\sigma_{t}}\right|_{c}+\left.\frac{Q_{m}}{\sigma_{t}}\right|_{\tilde{c}_{f e z}}\right)-\lambda_{m, \tilde{w}_{f e z}}^{f e z} \frac{\bar{A}_{f e p}, \bar{\Omega}_{m}}{3 \sigma_{t} V_{w f e z}}\left(\left.\frac{Q_{m}}{\sigma_{t}}\right|_{f}+\left.\frac{Q_{m}}{\sigma_{t}}\right|_{e}-\left.2 \frac{Q_{m}}{\sigma_{t}}\right|_{\tilde{c}_{f e z}}\right)
\end{array}\right. \\
& +\gamma\left(\tau_{m, \tilde{w}_{f e z}}\right)\left[\psi_{m, \tilde{w}_{f e z}}-\psi_{m, \tilde{w}_{f e z}}^{i n c}\right]-\frac{\vec{\Omega}_{m}}{\sigma_{t} V_{w}} \cdot\left(\vec{A}_{f e p^{\prime}, w} \psi_{m, f e p^{\prime}}+\vec{A}_{p^{\prime} e z, w} \psi_{m, p^{\prime} e z}+\vec{A}_{f p^{\prime} z, w} \psi_{m, f p^{\prime} z}\right), \quad \vec{A}_{f e z, w} \cdot \vec{\Omega}<0 .
\end{aligned}
$$

We have previously defined $\gamma\left(\tau_{m, f e z}\right)$ in equation (58), and $\psi_{m, w}^{i n c}$ and $\tau_{m, x x x}$ in equation (56).

These equations fully define our system. In practice the implementation details are a bit more complicated. The fully discretized system (energy, angle and space) is solved by employing a pair of embedded iteration loops. The inner iteration is used to converge the angular and angular flux moments for a single energy group. For this iteration the inscatter and distributed source values are fixed and we iterate only on the within group scattering source. In this iteration we form the total source (the RHS of the transport equation) from the sum of the fixed source (inscatter plus distributed sources) and the within-group scattering source. The within-group scattering source is estimated by using an initial guess (or previous iterate information) for the angular flux moments. With the RHS of our system fixed we sweep all wedges in the mesh for each angle in the quadrature set using the equations shown above. Once we have computed all of the new volume- and surface-averaged angular fluxes we update the corner-averaged angular flux moments and the scattering source and repeat the angle-wedge sweep until the angular flux moments are converged. This is a source-iteration (i.e. Richardson iteration) scheme. We repeat this iteration for each energy bin in our multi-group approximation. After we have computed all of the angular flux moments for all energy groups we rebuild the group to group scattering source and repeat the entire process until the angular flux moments converge. This procedure was implemented in the research transport code SNAC at Texas A\&M University. ${ }^{20}$

The 3D UCBL spatial discretization was designed to work on an unstructured mesh of polyhedra. We have also designed it to have good properties in the thick diffusive limit. It is conservative on corners and cells and is algebraically linear. The system has been designed to be an upstream method: we can sweep the mesh in a single direction without inverting any matrices to obtain volume or surface averaged angular fluxes. Lastly, it has been designed to minimize truncation error. In the next chapter we will examine all of 
these properties (and a few others) in greater detail to determine how successful we have been in designing a scheme that has all of the good properties listed in Chapter I. 


\title{
CHAPTER III
}

\begin{abstract}
ANALYSIS OF 3D UCBL
In this chapter, we examine the 3D UCBL spatial discretization scheme to measure how well it will performs in specific limits. These analyses will allow us to see if our method attains the goals that we set out to achieve. Our goal was a three-dimensional Corner Balance method with the following attractive properties (in order of priority): 1) robust and accurate solution in thick diffusive regions; 2) conservation on subcell (corner) volumes; 3) algebraic linearity, 4) positivity in source-free pure absorbers; 5) formulation that permits sweeping with no matrix inversions; 6) minimal spreading of a beam in a vacuum; and 7) $2^{\text {nd }}$ order or better truncation error.
\end{abstract}

\section{A. Three-dimensional Polyhedral Discretization}

The foremost goal of this work was to develop a spatial discretization scheme for the neutron transport equation that can take advantage of grids composed of arbitrarily connected polyhedra. As described in Chapter II, we have accomplished this by breaking polyhedral zones into corners and then breaking corners into wedges. Using tetrahedral wedges as the smallest volumetric element allowed us to devise a consistent and simple sweeping algorithm for polyhedral grids. This approach allows us to maintain particle balance on both zones and on corners. It also allowed us to construct a scheme that is algebraically linear and requires no matrix inversions as we sweep the mesh.

\section{B. 3D UCBL in the Thick Diffusion Limit}

The other primary goal of this work was to ensure that our spatial discretization had good performance characteristics for optically thick and highly scattering problems. It is clear from our work in Chapter II that this property was built into our spatial discretization scheme from the start. However, it is useful to complete a top-down asymptotic analysis for the 3D UCBL equations to ensure that we do capture the proposed solution in the thick diffusion limit. 
We will follow a standard procedure for this analysis. ${ }^{21}$ We first introduce a small scaling parameter, $\varepsilon$, into the transport equation so that when $\varepsilon$ becomes small our system becomes optically thick and highly scattering. This scaling is shown in equation (9). The scaled form of the 3D UCBL wedge balance equation (7), is shown here.

$$
\begin{aligned}
& \vec{A}_{f e z, w} \cdot \vec{\Omega}_{m}\left(\psi_{m, f e z}^{(0)}+\varepsilon \psi_{m, f e z}^{(1)}+\varepsilon^{2} \psi_{m, f e z}^{(2)}+\ldots\right)+\vec{A}_{f e p, w} \cdot \vec{\Omega}_{m}\left(\psi_{m, f e p}^{(0)}+\varepsilon \psi_{m, f e p}^{(1)}+\varepsilon^{2} \psi_{m, f e p}^{(2)}+\ldots\right) \\
& +\vec{A}_{f p z, w} \cdot \vec{\Omega}_{m}\left(\psi_{m, f p z}^{(0)}+\varepsilon \psi_{m, f p z}^{(1)}+\varepsilon^{2} \psi_{m, f p z}^{(2)}+\ldots\right)+\vec{A}_{p e z, w} \cdot \vec{\Omega}_{m}\left(\psi_{m, p e z}^{(0)}+\varepsilon \psi_{m, p e z}^{(1)}+\varepsilon^{2} \psi_{m, p e z}^{(2)}+\ldots\right) \\
& +\frac{\sigma_{t} V_{w}}{\varepsilon}\left(\psi_{m, w}^{(0)}+\varepsilon \psi_{m, w}^{(1)}+\varepsilon^{2} \psi_{m, w}^{(2)}+\ldots\right)=V_{w}\left[\frac{1}{4 \pi}\left(\frac{\sigma_{t}}{\varepsilon}-\varepsilon \sigma_{a}\right)\left(\phi_{c}^{(0)}+\varepsilon \phi_{c}^{(1)}+\varepsilon^{2} \phi_{c}^{(2)}+\ldots\right)+\frac{\varepsilon}{4 \pi} S_{0}^{e x t}\right]
\end{aligned}
$$

Following standard practice, we have also made the informed guess that the angular and scalar flux solutions are power series in $\varepsilon$ as shown in equation (10).

The remainder of our asymptotic analysis follows directly from our scaled equations and the power series expansion of our solution. Examining only the $O\left(\varepsilon^{-1}\right)$ terms of equation (2), we find that leading-order wedge-averaged angular fluxes are isotropic and are equal for all wedges in the same corner.

$$
\psi_{m, w}^{(0)}=\frac{1}{4 \pi} \phi_{c}^{(0)} .
$$

Using the scaling of the $Q / \sigma$ terms shown in equation (32), it follows from equation (66) that the $f p z$ and $p e z$ wedge-surface unknowns are also isotropic to leading order:

$$
\begin{aligned}
& \psi_{m, p e z}^{(0)}=\frac{1}{3} \frac{1}{4 \pi}\left(\phi_{e}^{(0)}+\phi_{z}^{(0)}+\phi_{c}^{(0)}\right), \\
& \psi_{m, f p z}^{(0)}=\frac{1}{3} \frac{1}{4 \pi}\left(\phi_{f}^{(0)}+\phi_{z}^{(0)}+\phi_{c}^{(0)}\right) .
\end{aligned}
$$

To arrive at this conclusion we expanded a scaled $\beta$ in a Taylor series expansion about $\varepsilon=0$. For this expansion the second term in both the $f p z$ and the pez closures is zero to $O\left(\varepsilon^{-1}\right)$.

$$
\beta(\tau)=\left[1+\frac{1}{3} \frac{\tau}{\varepsilon}+\frac{1}{18}\left(\frac{\tau}{\varepsilon}\right)^{2}\right]^{-1}=18\left(\frac{\varepsilon}{\tau}\right)^{2}-108\left(\frac{\varepsilon}{\tau}\right)^{3}+324\left(\frac{\varepsilon}{\tau}\right)^{4}+O\left(\varepsilon^{6}\right) .
$$

To evaluate the $f e z, p e z$ and $f p z$ closures the closure we need the following Taylor series expansions for both $\chi\left(\tau_{m, x x x}\right)$ and $\lambda_{m, w}^{x x x}$ where $x x x \in\{f e z, p e z, f p z\}$.

$$
\begin{aligned}
& \gamma\left(\tau_{m, x x x}\right)=\frac{\varepsilon^{2}}{\tau_{m, x x x}^{2}}+\frac{\varepsilon^{3}}{4 \tau_{m, x x x}^{3}}+O\left(\varepsilon^{4}\right), \\
& \lambda_{m, w}^{x x x}=1-\frac{\left(\vec{A}_{x x x, w} \cdot \vec{\Omega}_{m}\right)^{2}}{\sigma_{t}^{2} V_{w}^{2}} \varepsilon^{2}+O\left(\varepsilon^{4}\right) .
\end{aligned}
$$

Substituting these two expansions into equation (67), we find that to leading order the $f e z$ wedge-surface unknown is also isotropic.

$$
\psi_{m, f e z}^{(0)}=\frac{1}{2} \frac{1}{4 \pi}\left(\phi_{\tilde{c}_{f e z}}^{(0)}+\phi_{c}^{(0)}\right)
$$


The fep step closure (24) causes this surface unknown to be half-range isotropic to leading order in the interior of the problem. On the problem boundary, $\vec{r} \in \Gamma$, the fep surface unknown is defined by the boundary condition.

$$
\psi_{m, f e p}^{(0)}= \begin{cases}\frac{1}{4 \pi} \phi_{c}^{(0)} & \vec{A}_{\text {fep }, w} \cdot \vec{\Omega}_{m}>0, \\ \frac{1}{4 \pi} \phi_{\tilde{c}_{\text {fep }}}^{(0)} & \vec{A}_{\text {fep }, w} \cdot \vec{\Omega}_{m}<0, \quad \vec{r} \notin \Gamma, \\ \psi_{m, \text { fep }}^{\text {inc }} & \vec{A}_{\text {fep }, w} \cdot \vec{\Omega}_{m}<0, \quad \vec{r} \in \Gamma .\end{cases}
$$

This portion of the analysis has shown that to leading-order all of the volumetric- and surface-averaged angular fluxes will be isotropic in the interior of the problem. This is required if the 3D UCBL spatial discretization is to capture a diffusion solution in the thick limit. We will now show how our volumecentered discretization can have the same leading-order solution as a vertex-centered diffusion scheme.

The $O(1)$ terms of equation (68) can be summed for all wedges in a corner to produce a corner-balance equation. Remember that the $f p z$ and pez terms will cancel out with each other.

$$
\sum_{w \in c}\left[\vec{A}_{f e z, w} \cdot \vec{\Omega}_{m} \psi_{m, f e z}^{(0)}+\vec{A}_{f e p, w} \cdot \vec{\Omega}_{m} \psi_{m, f e p}^{(0)}\right]+V_{c} \sigma_{t} \sum_{w \in c} \psi_{m, w}^{(1)}=\frac{1}{4 \pi} V_{c} \sigma_{t} \phi_{c}^{(1)} .
$$

Now we can integrate this corner-balance equation over all directions to give:

$$
\sum_{w \in C} \sum_{m} w_{m}\left[\vec{A}_{f e z, w} \cdot \vec{\Omega}_{m} \psi_{m, f e z}^{(0)}+\vec{A}_{f e p, w} \cdot \vec{\Omega}_{m} \psi_{m, f e p}^{(0)}\right]=0
$$

The last two terms cancel, and if the quadrature set properly integrates linear functions of $\vec{\Omega}$, the $f e z$ terms will vanish because these surface intensities are isotropic to leading order.

$$
\sum_{w \in c} \vec{A}_{f e p, w} \cdot \sum_{m} w_{m} \vec{\Omega}_{m} \psi_{m, f e p}^{(0)}=0
$$

Away from boundaries we can use equation (4) to show that the leading-order corner-averaged scalar fluxes around a common vertex are equal.

$$
\sum_{w \in c}\left(\phi_{c}^{(0)}-\phi_{\tilde{c}_{f e p}}^{(0)}\right) \vec{A}_{f e p, w} \cdot \sum_{\vec{A}_{f e p, w} \cdot{ }^{-}{ }_{m}>0} w_{m} \vec{\Omega}_{m}=0 \quad \text { so that } \quad \phi_{c}^{(0)}=\phi_{\tilde{c}_{f e p}}^{(0)} \equiv \phi_{p}^{(0)} \quad \forall c, \tilde{c}_{f e p} \in p . \quad \text { (28) \& }
$$

The details of this conclusion are discussed in more detail in Appendix A. This result tells us that the leading-order corner-averaged angular fluxes are continuous at interior vertices. This result is important because it allows us to conceptualize a dual grid whose cells are centered at the vertices of the real grid as shown in Figure 3. It also greatly reduces the number of independent unknowns in the leading-order solution giving it a chance to satisfy a reasonably simple diffusion discretization. The leading-order solution lives on this shifted grid, which is a vertex-centered grid that is consistent with the grid that Palmer's discrete diffusion solution uses.

Now we need to examine the behavior of our scheme near the problem boundary. Equations (53) and (54) specify our solution, $\phi_{\tilde{c}_{\text {fep }}}^{(0)}$, on the problem boundary.

$$
\phi_{\tilde{c}_{\text {fep }}}^{(0)}=\frac{2}{\rho} \sum_{\vec{n} \cdot \bar{\Omega}_{m}<0} w_{m} 2\left|\vec{n} \cdot \vec{\Omega}_{m}\right| \psi_{m, f e p}^{\text {inc,(0) }}, \quad \vec{r} \in \Gamma .
$$




$$
\begin{aligned}
& \vec{n}=\frac{\vec{A}_{f e p, w}}{\left|\vec{A}_{f e p, w}\right|}, \\
& \rho=\frac{1}{\pi} \sum_{\vec{n} \cdot \vec{\Omega}>0} w_{m}(\vec{n} \cdot \vec{\Omega})=\frac{1}{\pi} \sum_{\vec{n} \cdot \vec{\Omega}<0} w_{m}|\vec{n} \cdot \vec{\Omega}| \approx 1 .
\end{aligned}
$$

As stated in Section II.F., this is a discrete Marshak quantity. That is, it is a cosine-weighted integral of incident intensities. It should be noted that this type of boundary condition might produce boundary layer solutions that are in error by up to a factor of two.

To this point we have shown that our discretization produces an isotropic solution on a vertex-centered grid to leading order. We now show that in this limit it captures the same balance equation and leakage used in Palmer's discretization. We obtain a statement of particle balance for each wedge by integrating the $O\left(\varepsilon^{1}\right)$ terms of equation (68) over all directions.

$$
\vec{A}_{f e z, w} \cdot \vec{J}_{m, f e z}^{(1)}+\vec{A}_{f e p, w} \cdot \vec{J}_{m, f e p}^{(1)}+\vec{A}_{f p z, w} \cdot \vec{J}_{m, f p z}^{(1)}+\vec{A}_{p e z, w} \cdot \vec{J}_{m, p e z}^{(1)}+\sigma_{a} V_{w} \phi_{p}^{(0)}=V_{w} S_{0}^{e x t} .
$$

This equation represents the net out-leakage rate for each of the four wedge faces and the total absorption rate equaling the total source rate in a wedge. This is the desired diffusion balance equation. In this equation, we have defined the $O\left(\mathcal{E}^{1}\right)$ net current densities to be

$$
\vec{J}_{m, x x x}^{(1)}=\sum_{m} w_{m} \vec{\Omega}_{m} \psi_{m, x x x}^{(1)}, \quad \text { for } x x x \in\{f e p, f e z, f p z, p e z\} .
$$

If we sum equation (77) over all the wedges that touch a given interior vertex, we obtain a balance equation for a dual cell:

$$
\sum_{w \in p}\left(\vec{A}_{f e z, w} \cdot \vec{J}_{m, f e z}^{(1)}\right)+\phi_{p}^{(0)} \sum_{w \in p} \sigma_{a} V_{w}=\sum_{w \in p} V_{w} S_{0}^{e x t} .
$$

Note that the $f e p, f p z$ and $p e z$ currents cancel out since the only wedge face on the surface of the dual cell is the $f e z$ face. Additionally we have used the notation $w \in p$ to represent all the wedges that have point $p$ as one of their vertices. We have also used equation (29) to pull $\phi_{p}^{(0)}$ outside of the sum.

Equation (79) tells us that 3D UCBL preserves particle balance on a dual cell in the diffusive limit. This was no surprise, since the method preserves balance on each individual corner. To fully describe how this scheme behaves in thick diffusive problems we must relate the net current density on the dual cell surfaces $\left(\vec{J}_{m, f e z}^{(1)}\right)$ to the dual cell volume-averaged scalar fluxes $\left(\phi_{p}^{(0)}\right)$. To find this relationship we examine equation (78) for the $f e z$ net current density. Since this $f e z$ current depends on the $f e z$ surface-averaged angular flux we start with the $O(\varepsilon)$ equation for this quantity.

$$
\begin{aligned}
& \psi_{m, f e z}^{(1)}=\frac{1}{2} \frac{1}{4 \pi}\left(\phi_{\tilde{c}_{f e z}}^{(1)}+\phi_{c}^{(1)}\right)+\frac{1}{2} \frac{1}{4 \pi} \frac{\left|\bar{\Omega}_{\Omega^{m}} \cdot \bar{A}_{f e z, w}\right|}{\sigma_{t} t_{w}}\left(\phi_{\tilde{c}_{f f z}}^{(0)}+\phi_{c}^{(0)}\right)
\end{aligned}
$$

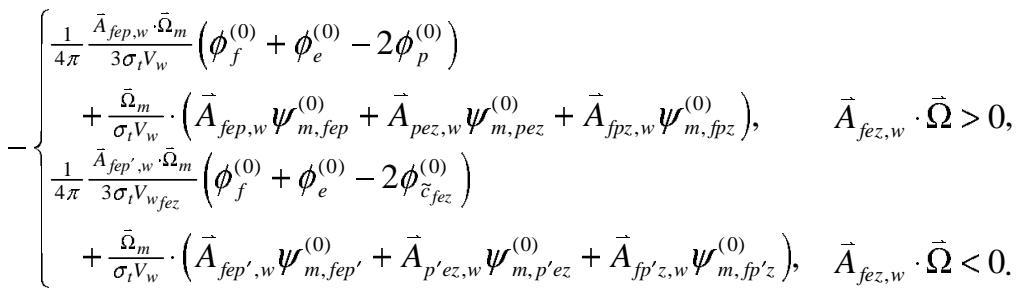


We will simplify this closure before applying it to equation (78). If the wedge of interest is in the problem interior, then each wedge-surface angular intensity in this equation is isotropic as we saw in equations (70), (73) and (34). Substituting these definitions into equation (80), after a bit of manipulation we arrive at the following equation for the $O(\varepsilon) f e z$ angular flux:

$$
\begin{aligned}
\psi_{m, f e z}^{(1)}=\frac{1}{2} \frac{1}{4 \pi}\left(\phi_{\tilde{c}_{f e z}}^{(1)}+\phi_{c}^{(1)}\right)+\frac{1}{2} \frac{1}{4 \pi} \frac{\left|\vec{\Omega}_{m} \cdot \vec{A}_{f e z, w}\right|}{\sigma_{t} t_{w}}\left(\phi_{\tilde{c}_{f e z}}^{(0)}+\phi_{c}^{(0)}\right) \\
-\frac{1}{4 \pi} \frac{1}{3} \frac{\bar{\Omega}_{m}}{\sigma_{t} t_{w}} \cdot\left\{\begin{array}{l}
{\left[\begin{array}{l}
\vec{A}_{f e p, w}\left(\phi_{f}^{(0)}+\phi_{e}^{(0)}+\phi_{p}^{(0)}\right)+\vec{A}_{p e z, w}\left(\phi_{e}^{(0)}+\phi_{z}^{(0)}+\phi_{c}^{(0)}\right) \\
+\vec{A}_{f p z, w}\left(\phi_{f}^{(0)}+\phi_{z}^{(0)}+\phi_{c}^{(0)}\right)
\end{array}\right], \quad \vec{A}_{f e z, w} \cdot \vec{\Omega}>0, r \notin \Gamma,} \\
{\left[\begin{array}{l}
\vec{A}_{f e p^{\prime}, w}\left(\phi_{f}^{(0)}+\phi_{e}^{(0)}+\phi_{\tilde{c}_{f e z}}^{(0)}\right)+\vec{A}_{p^{\prime} e z, w}\left(\phi_{e}^{(0)}+\phi_{z}^{(0)}+\phi_{\tilde{c}_{f e z}}^{(0)}\right) \\
+\vec{A}_{f p^{\prime} z, w}\left(\phi_{f}^{(0)}+\phi_{z}^{(0)}+\phi_{\tilde{c}_{f e z}}^{(0)}\right)
\end{array}\right], \quad \vec{A}_{f e z, w} \cdot \vec{\Omega}<0, r \notin \Gamma .}
\end{array}\right.
\end{aligned}
$$

We have used the notation $p^{\prime}$ to represent the vertex that is associated with $\tilde{c}_{f e z}$ (see Figure 4). The term $\tilde{c}_{f e z}$ refers to the corner that shares the $f e z$ face with the current corner. Because the corner scalar fluxes are all equal to leading order about a common vertex we may use these two symbols interchangeably so that $\phi_{\tilde{c}_{f e z}}^{(0)}=\phi_{p^{\prime}}^{(0)}$.

With $\psi_{m, f e z}^{(1)}$ defined we may now proceed by constructing the net current density on the boundary of the dual cell. Using equation (81) in equation (78) for the $f e z$ closure and simplifying we find:

$$
\begin{aligned}
& \vec{A}_{f e z, w} \cdot \vec{J}_{m, f e z}^{(1)}=\vec{A}_{f e z, w} \cdot \sum_{\bar{A}_{f e z, w} \cdot \mathbf{\Omega}_{m}>0} w_{m} \vec{\Omega}_{m} \psi_{m, f e z}^{(1)}+\vec{A}_{f e z, w} \cdot \sum_{\bar{A}_{f e z, w} \cdot \mathbf{\Omega}_{m}<0} w_{m} \vec{\Omega}_{m} \psi_{m, f e z}^{(1)} \\
& =\frac{1}{4 \pi} \frac{1}{2}\left(\phi_{\tilde{c}_{f e z}}^{(1)}+\phi_{c}^{(1)}\right) \vec{A}_{f e z, w} \cdot \sum_{m} w_{m} \vec{\Omega}_{m}+\frac{1}{4 \pi} \frac{1}{2 \sigma_{t} V_{w}}\left(\phi_{\tilde{c}_{f e z}}^{(0)}+\phi_{c}^{(0)}\right) \vec{A}_{f e z, w} \cdot \sum_{m} w_{m} \vec{\Omega}_{m}\left|\vec{\Omega}_{m} \cdot \vec{A}_{f e z, w}\right|
\end{aligned}
$$

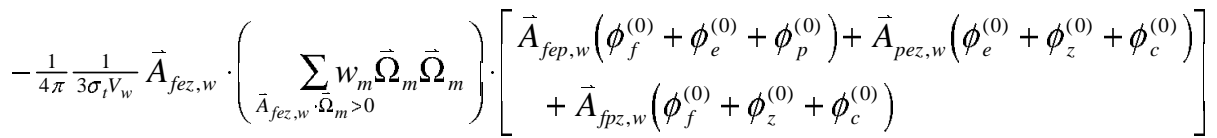

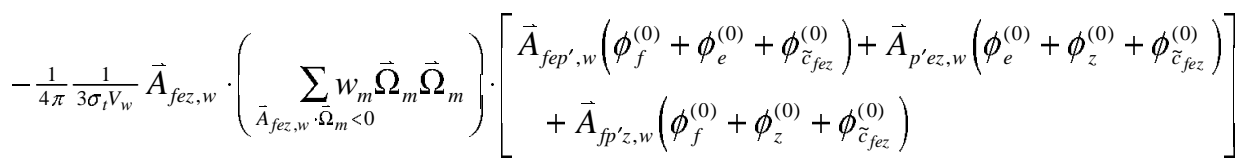

The first term will evaluate to zero if the quadrature set is symmetric and integrates linear functions of $\vec{\Omega}$ correctly. The second term will integrate to zero because it is an odd function in $\vec{\Omega}$. Assuming that the half-range sums of the $\vec{\Omega}_{m} \vec{\Omega}_{m}$ tensor correctly evaluate to $2 \pi / 3$ then our expression for the net current density on the dual-cell boundary reduces to the following expression.

$$
\vec{A}_{f e z, w} \cdot \vec{J}_{m, f e z}^{(1)}=\frac{-1}{6 \sigma_{t} V_{w}} \vec{A}_{f e z, w} \cdot\left[\begin{array}{l}
\frac{1}{3} \vec{A}_{f e p, w}\left(\phi_{f}^{(0)}+\phi_{e}^{(0)}+\phi_{p}^{(0)}\right)+\frac{1}{3} \vec{A}_{f e p^{\prime}, w}\left(\phi_{f}^{(0)}+\phi_{e}^{(0)}+\phi_{\tilde{c}_{f e z}}^{(0)}\right) \\
+\frac{1}{3} \vec{A}_{p e z, w}\left(\phi_{e}^{(0)}+\phi_{z}^{(0)}+\phi_{c}^{(0)}\right)+\frac{1}{3} \vec{A}_{p^{\prime} e z, w}\left(\phi_{e}^{(0)}+\phi_{z}^{(0)}+\phi_{\tilde{c}_{f e z}}^{(0)}\right) \\
+\frac{1}{3} \vec{A}_{f p z, w}\left(\phi_{f}^{(0)}+\phi_{z}^{(0)}+\phi_{c}^{(0)}\right)+\frac{1}{3} \vec{A}_{f p^{\prime} z, w}\left(\phi_{f}^{(0)}+\phi_{z}^{(0)}+\phi_{\tilde{c}_{f e z}}^{(0)}\right)
\end{array}\right]
$$

This equation is equivalent to that used in Palmer's diffusion discretization. Thus, our leading-order UCBL solution does satisfy Palmer's vertex-centered discrete diffusion discretization. 
Let us summarize our diffusion-limit results. In the interior of the problem, the leading-order 3D UCBL angular fluxes in a thick diffusive region are isotropic. The corner average scalar fluxes are equal, to leading order, in all corners about a given interior vertex. This union of corners forms a dual cell on which the method satisfies conservation of particles. In addition to this equation of balance the scheme also captures the same discrete representation of Fick's Law as Palmer's discretization. Additionally, near problem boundaries, we have shown that to leading order our scheme satisfies a Marshak-weighted boundary condition in the thick diffusion limit.

An important consequence of this result is that the 3D UCBL solution will be exact to leading order if the exact diffusion solution is a linear function of position. This follows from Palmer's scheme that exactly captures linear solutions.

\section{C. Upstream Nature of $3 D$ UCBL}

Another goal of our discretization was to create a sweeping scheme that is completely "upstream." That is, we wanted to avoid inverting any matrices to find the solution in each cell as we sweep the spatial mesh in one discrete ordinate direction. This allows us to compute all of the needed information about the current wedge by using only information that has already been computed in wedges that are upstream from the wedge of interest as well as sources, which are assumed known during the sweep. To demonstrate this we examine a single wedge and the algorithm used to find the volumetric- and surface-averaged angular fluxes and the volumetric-averaged scalar fluxes. Consider the wedge shown in Figure 6 oriented so that the discrete ordinate angle of interest is directed into the page. With this perspective, our wedge has two faces ( $p e z$ and $f e z$ ) for which the angular flux is incident and two exiting faces (fep and $f p z$ ).

We can write the system of equation specified by (7), (24), (66) and (67) in the upstream form that is used when implementing this scheme in the mesh sweeper of a transport code. We substitute the closure equations (24), (66) and (67) into the wedge balance equation (7) to eliminate all of the exiting surfaceaveraged terms (for our example we eliminate $\psi_{m, f p z}$ and $\psi_{m, f e p}$ ). After a considerable amount of algebra we arrive at an expression for the wedge-averaged angular flux based only on incident information.

$$
\psi_{m, w}=D_{m, w}^{-1}\left(I_{m, w}+S_{m, w}\right),
$$

where the term $I_{m, w}$ represents the incident information, $S_{m, w}$ represents sources internal to the wedge (volumetric sources plus inscatter). $D_{m, w}$ is a parameter that represents the total collision rate and the net leakage rate from the wedge. We define these three new parameters here. 


$$
\begin{aligned}
& D_{m, w}=1+\gamma\left(\tau_{m, f e z}\right) \frac{\vec{A}_{f e z, w} \cdot \vec{\Omega}_{m}+\left|\vec{A}_{f e z, w} \cdot \vec{\Omega}_{m}\right|}{2\left(\sigma_{t} V_{w}+\vec{A}_{f e z, w} \cdot \vec{\Omega}_{m}\right)} \\
& +\frac{1}{2 \sigma_{t} V_{w}}\left[1-\frac{\vec{A}_{f e z, w} \cdot \vec{\Omega}_{m}+\left|\vec{A}_{f e z, w} \cdot \vec{\Omega}_{m}\right|}{2\left(\sigma_{t} V_{w}+\vec{A}_{f e z, w} \cdot \vec{\Omega}_{m}\right)}\right] \cdot\left[\begin{array}{l}
\left(\left|\vec{A}_{f e p, w} \cdot \vec{\Omega}_{m}\right|+\vec{A}_{f e p, w} \cdot \vec{\Omega}_{m}\right) \\
+\left(1+\beta\left(\tau_{m, p e z}\right)\right) \cdot\left(\left|\vec{A}_{p e z, w} \cdot \vec{\Omega}_{m}\right|+\vec{A}_{p e z, w} \cdot \vec{\Omega}_{m}\right) \\
+\left(1+\beta\left(\tau_{m, f p z}\right)\right) \cdot\left(\left|\vec{A}_{f p z, w} \cdot \vec{\Omega}_{m}\right|+\vec{A}_{f p z, w} \cdot \vec{\Omega}_{m}\right)
\end{array}\right], \\
& \left.I_{m, w}=\frac{1}{2 \sigma_{t} V_{w}}\left[1-\frac{\vec{A}_{f e z, w} \cdot \vec{\Omega}_{m}+\left|\vec{A}_{f e z, w} \cdot \vec{\Omega}_{m}\right|}{2\left(\sigma_{t} V_{w}+\vec{A}_{f e z, w} \cdot \vec{\Omega}_{m}\right)}\right] \cdot\left[\begin{array}{l}
\left(\left|\vec{A}_{f e p, w} \cdot \vec{\Omega}_{m}\right|-\vec{A}_{f e p, w} \cdot \vec{\Omega}_{m}\right) \psi_{m, f e p}^{i n c} \\
+\left(\left|\vec{A}_{p e z, w} \cdot \vec{\Omega}_{m}\right|-\vec{A}_{p e z, w} \cdot \vec{\Omega}_{m}\right) \psi_{m, p e z}^{i n c} \\
+\left(\left|\vec{A}_{f p z, w} \cdot \vec{\Omega}_{m}\right|-\vec{A}_{f p z, w} \cdot \vec{\Omega}_{m}\right) \psi_{m, f p z}^{i n c} \\
\left(\begin{array}{l}
\left(\tau_{m, p e z}\right)\left(\left|\vec{A}_{p e z, w} \cdot \vec{\Omega}_{m}\right|+\vec{A}_{p e z, w} \cdot \vec{\Omega}_{m}\right) \\
+\beta\left(\tau_{m, f p z}\right)\left(\left|\vec{A}_{f p z, w} \cdot \vec{\Omega}_{m}\right|+\vec{A}_{f p z, w} \cdot \vec{\Omega}_{m}\right)
\end{array}\right)
\end{array}\right] \psi_{m, w}^{i n c}\right) \\
& +\gamma\left(\tau_{m, f e z}\right)\left(\frac{\vec{A}_{f e z, w} \cdot \vec{\Omega}_{m}+\left|\vec{A}_{f e z, w} \cdot \vec{\Omega}_{m}\right|}{2\left(\sigma_{t} V_{w}+\vec{A}_{f e z, w} \cdot \vec{\Omega}_{m}\right)}\right) \psi_{m, w}^{i n c}+\frac{1}{2 \sigma_{t} V_{w}}\left(\left|\vec{A}_{f e z, w} \cdot \vec{\Omega}_{m}\right|-\vec{A}_{f e z, w} \cdot \vec{\Omega}_{m}\right) \psi_{m, f e z}^{i n c}, \\
& S_{m, w}=\left.\frac{Q_{m}}{\sigma_{t}}\right|_{c}-\left.\frac{\vec{A}_{f e z, w} \cdot \vec{\Omega}_{m}+\left|\vec{A}_{f e z, w} \cdot \vec{\Omega}_{m}\right|}{2\left(\sigma_{t} V_{w}+\vec{A}_{f e z, w} \cdot \vec{\Omega}_{m}\right)} \frac{Q_{m}}{\sigma_{t}}\right|_{e} \\
& +\frac{\vec{A}_{f e z, w} \cdot \vec{\Omega}_{m}+\left|\vec{A}_{f e z, w} \cdot \vec{\Omega}_{m}\right|}{2\left(\sigma_{t} V_{w}+\vec{A}_{f e z, w} \cdot \vec{\Omega}_{m}\right)} \frac{\left.\vec{A}_{f e p, w} \cdot \vec{\Omega}_{m}\right)}{3 \sigma_{t} V_{w}}\left[\left.\frac{Q_{m}}{\sigma_{t}}\right|_{f}+\left.\frac{Q_{m}}{\sigma_{t}}\right|_{e}-\left.2 \frac{Q_{m}}{\sigma_{t}}\right|_{c}\right] \\
& +\frac{1}{6 \sigma_{t} V_{w}}\left[\frac{\vec{A}_{f e z, w} \cdot \vec{\Omega}_{m}+\left|\vec{A}_{f e z, w} \cdot \vec{\Omega}_{m}\right|}{2\left(\sigma_{t} V_{w}+\vec{A}_{f e z, w} \cdot \vec{\Omega}_{m}\right)}-1\right] \cdot\left[\begin{array}{l}
\left(\left|\vec{A}_{p e z, w} \cdot \vec{\Omega}_{m}\right|+\vec{A}_{p e z, w} \cdot \vec{\Omega}_{m}\right)\left(\left.\frac{Q_{m}}{\sigma_{t}}\right|_{e}+\left.\frac{Q_{m}}{\sigma_{t}}\right|_{z}-\left.2 \frac{Q_{m}}{\sigma_{t}}\right|_{c}\right) \\
+\left(\left|\vec{A}_{f p z, w} \cdot \vec{\Omega}_{m}\right|+\vec{A}_{f p z, w} \cdot \vec{\Omega}_{m}\right)\left(\left.\frac{Q_{m}}{\sigma_{t}}\right|_{f}+\left.\frac{Q_{m}}{\sigma_{t}}\right|_{z}-\left.2 \frac{Q_{m}}{\sigma_{t}}\right|_{c}\right)
\end{array}\right] .
\end{aligned}
$$

The source and inleakage information is known before equation (83) is evaluated for this wedge. The loss operator only depends on geometrical and material properties that are computed as known information. Terms of the form $\left(\left|\vec{A}_{x x x, w} \cdot \vec{\Omega}_{m}\right| \pm \vec{A}_{x x x, w} \cdot \vec{\Omega}_{m}\right)$ are used to ensure that local information is only used for exiting directions (“-”) or only for incoming directions (“+”). Some of the parameters ( $\lambda_{m, w}, \gamma_{m, w} \beta_{m, w}, \psi_{m, w}^{i n c}$ and the $Q_{m} / \sigma_{t}$ terms) used in these equation are not specified here because they were defined in Chapter II (see equations (45), (56), (58), (62) and (63)-(65)). When examining equations (84)-(86) it is important to realize that all of the terms are either known are have a zero value. Thus, when evaluating the volumeaveraged angular flux for a wedge, $D_{m, w}, I_{m, w}$ and $S_{m, w}$ will be defined purely from upstream or known information.

Once $\psi_{m, w}$ is computed from equation (83) then all of the exiting surface-averaged unknowns may be computed using the closure equations (24), (66) and (67). These closure equations depend only on upstream 
information including the volume-averaged wedge angular flux, incident surface-averaged angular fluxes and geometric and material properties of the wedge. That is, the closures use only upstream and known source information.

To better understand the upstream nature of our equations it is useful to evaluate equations (84)-(86) for the wedge orientation shown in Figure 6. Examining this simplified problem may help the reader visualize the impact of the upstream nature of our system. The equations (84)-(86) simplify when evaluated for a wedge whose orientation matches the wedge form Figure 6. This simplification occurs because we know the sign of each area-projection term. This knowledge allows many terms in these equations to cancel out as shown below.

$$
\begin{aligned}
& D_{m, w}=1+\frac{1}{\sigma_{t} V_{w}}\left[\left(\vec{A}_{f e p, w} \cdot \vec{\Omega}_{m}\right)+\left(\vec{A}_{f p z, w} \cdot \vec{\Omega}_{m}\right) \cdot\left(1+\beta\left(\tau_{m, f p z}\right)\right)\right], \\
& I_{m, w}=\frac{1}{\sigma_{t} V_{w}}\left[\begin{array}{c}
\left|\vec{A}_{p e z, w} \cdot \vec{\Omega}_{m}\right| \psi_{m, p e z}^{i n c}+\left|\vec{A}_{f e z, w} \cdot \vec{\Omega}_{m}\right| \psi_{m, f e z}^{i n c} \\
+\left(\vec{A}_{f p z, w} \cdot \vec{\Omega}_{m}\right) \beta\left(\tau_{m, f p z}\right) \psi_{m, w}^{i n c}
\end{array}\right], \\
& \left.S_{m, w}=\frac{Q_{m}}{\sigma_{t}} \mid-\frac{\left(\vec{A}_{f p z, w} \cdot \vec{\Omega}_{m}\right)}{3 \sigma_{t} V_{w}}\left(\frac{Q_{m}}{\sigma_{t}}\left|+\frac{Q_{m}}{\sigma_{t}}\right|_{z}-2 \frac{Q_{m}}{\sigma_{t}} \mid\right) \quad\right\} w \text { from Figure } 6 .
\end{aligned}
$$

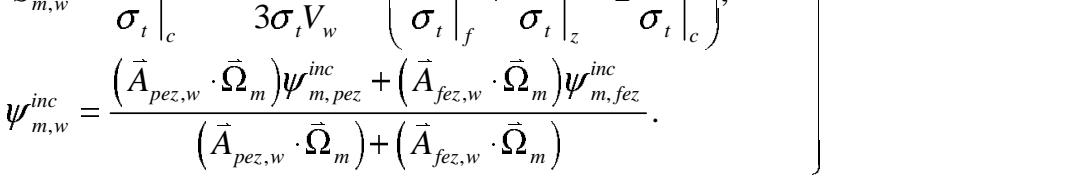

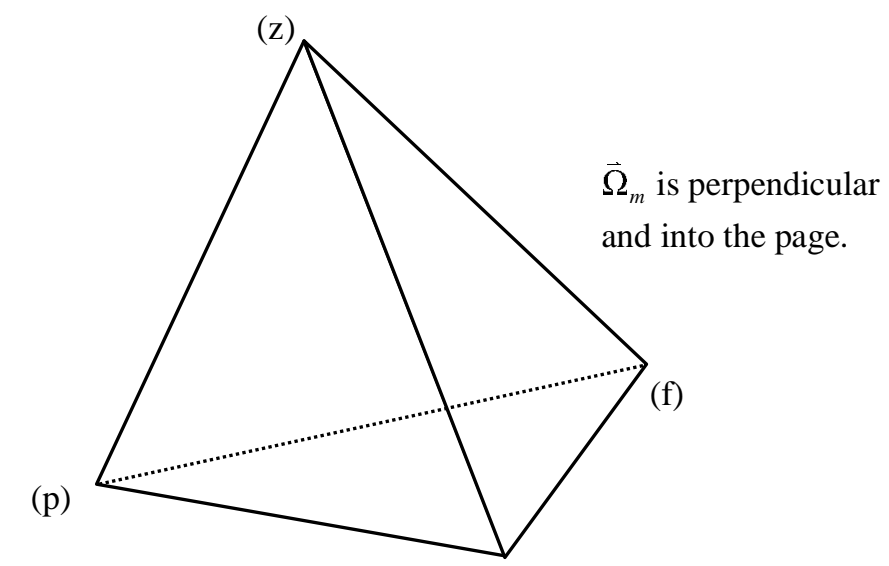

(e)

Figure 6. A single tetrahedral wedge.

For our particular case the volume-averaged angular flux for this wedge depends only on the surfaceaveraged angular flux for the $f e z$ and $p e z$ faces (plus internal sources). These two values are known because they are upstream of our wedge and have already been computed as exiting values from those upstream 
wedges. In return, after the wedge-averaged angular flux has been determined then we can use the closure equations to determine what the surface-averaged angular fluxes are for the $f p z$ and $f e p$ exiting faces.

While this example only demonstrates the upstream nature of our scheme for wedges oriented with respect to $\vec{\Omega}_{m}$ in the same way as the wedge in Figure 6, a similar simplification may be exercised on equations (83)-(86) for other orientations. Such a procedure would verify that 3D UCBL is upstream for all wedge orientations.

In this section we have shown that $3 \mathrm{D}$ UCBL is a purely upstream scheme. The primary advantage of this property is that we can sweep the spatial mesh in a single direction solving for the volume-averaged angular flux in each cell without performing any computationally slow matrix inversions.

\section{D. Positivity in a Source-Free Purely Absorbing Media}

Since negative flux values are non-physical for the analytic transport equation, it is desirable for our discretized system to posses this same property. While we do not attempt to guarantee that our scheme is purely positive for all problems we can ensure that it will always produce positive solutions in a source-free purely absorbing media. If we examine the equations (83)-(86) for such a problem we notice the following properties about our equations:

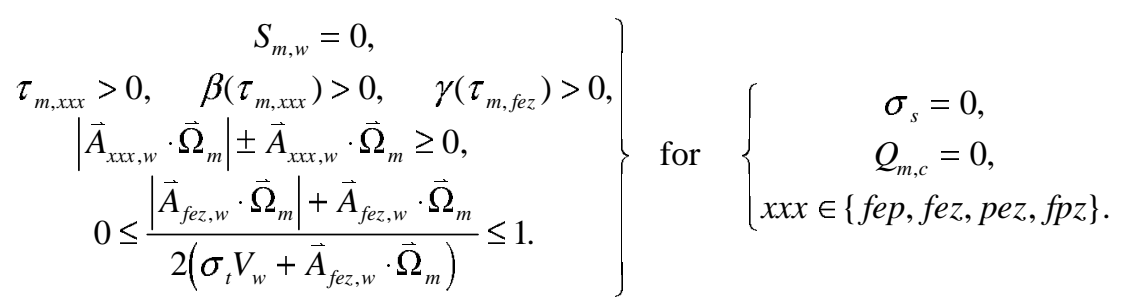

The source term goes to zero because there are no distributed sources (fission or other volumetric sources) and there is no scattering. By definition $\tau, \beta$ and $\gamma$ are purely positive. The sum/difference of the area dot omega terms will be positive or zero. Additionally, the last term in equation (88) is also bounded by the interval $(0,1)$. One result of these observations is that $D_{m, w}$ is strictly positive which implies that $D_{m, w}^{-1}$ will

also be positive. $I_{m, w}$ is guaranteed to be non-negative if the incident fluxes $\left\{\psi_{m, f e p}^{i n c}, \psi_{m, f e z}^{i n c}, \psi_{m, p e z}^{i n c}, \psi_{m, f p z}^{i n c}\right\}$ are all non-negative.

So, given non-negative incident information, a purely absorbing media and no distributed sources our method will preserve a non-negative solution for each wedge. If all of the wedge angular fluxes are positive then the corner and zone angular and scalar fluxes will also be positive.

$$
\psi_{m, w}=D_{m, w}^{-1} I_{m, w} \geq 0 \text { for }\left\{\begin{array}{cl}
\sigma_{s}=0, & Q_{m, c}=0, \\
\psi_{m, \text { fep }}^{i n c} \geq 0, & \psi_{m, f e z}^{i n c} \geq 0, \\
\psi_{m, p e z}^{i n c} \geq 0, & \psi_{m, f p z}^{i n c} \geq 0 .
\end{array}\right.
$$


This equation only holds for a single wedge. We must ensure that the exiting surface-averaged angular fluxes from this wedge are purely positive before we can say that our method produces a non-negative solution for these types of problems. Examining in this context the closure equations (24), (66) and (67) from Chapter II, we note that for exiting directions they reduce to the following form:

$$
\left.\begin{array}{ll}
\psi_{m, f e p}=\psi_{m, w}, & \vec{A}_{f e p, w} \cdot \vec{\Omega}_{m}>0, \\
\psi_{m, p e z}=\psi_{m, w}+\beta\left(\tau_{m, p e z}\right)\left[\psi_{m, w}-\psi_{m, w}^{i n c}\right], & \vec{A}_{p e z, w} \cdot \vec{\Omega}_{m}>0, \\
\psi_{m, f p z}=\psi_{m, w}+\beta\left(\tau_{m, f p z}\right)\left[\psi_{m, w}-\psi_{m, w}^{i n c}\right], & \vec{A}_{f p z, w} \cdot \vec{\Omega}_{m}>0, \\
\psi_{m, f e z}=\psi_{m, w}+\gamma\left(\tau_{m, f e z}\right)\left[\psi_{m, w}-\psi_{m, w}^{i n c}\right], & \vec{A}_{f e z, w} \cdot \vec{\Omega}_{m}>0,
\end{array}\right\} \text { for }\left\{\begin{array}{r}
\sigma_{s}=0, \\
Q_{m, c}=0 .
\end{array}\right.
$$

Now consider the wedge balance equation (see equation (7)). For a source free problem the right-handside, RHS, is zero and we can divide through by $\sigma_{t} V_{w}$ to arrive at a simplified form for the balance equation.

$$
\psi_{m, w}+\frac{1}{\sigma_{t} V_{w}} \sum_{\vec{A}_{x x x} \cdot \bar{\Omega}_{m}>0}\left(\vec{A}_{x x x} \cdot \vec{\Omega}_{m}\right) \psi_{m, x x x}=\frac{1}{\sigma_{t} V_{w}} \sum_{\vec{A}_{x x} \cdot \vec{\Omega}_{m}<0}\left|\vec{A}_{x x x} \cdot \vec{\Omega}_{m}\right| \psi_{m, x x x}, \quad x x x \in\{\text { fep, fez, fp z, pez }\} .
$$

When can use the definition of $\psi_{m, w}^{i n c}$ from equation (56) to further simplify the RHS.

$$
\frac{1}{\sigma_{t} V_{w}} \sum_{\vec{A}_{x x x}, \bar{\Omega}_{m}<0}\left|\vec{A}_{x x x} \cdot \vec{\Omega}_{m}\right| \psi_{m, x x x}=\psi_{m, w}^{i n c} \sum_{\vec{A}_{x x x}, \bar{\Omega}_{m}<0} \frac{\left|\vec{A}_{x x x} \cdot \vec{\Omega}_{m}\right|}{\sigma_{t} V_{w}} .
$$

Since the net projected surface area must be zero for each wedge we have

$$
\sum_{\vec{A}_{x x x} \cdot \vec{\Omega}_{m}<0}\left|\vec{A}_{x x x} \cdot \vec{\Omega}_{m}\right|=\sum_{\vec{A}_{x x x} \cdot \vec{\Omega}_{m}>0}\left|\vec{A}_{x x x} \cdot \vec{\Omega}_{m}\right| \cdot
$$

Using this equality and the definition of the optical thickness to modify equation (91) we have the following wedge balance equation.

$$
\psi_{m, w}+\sum_{\bar{A}_{x x x} \cdot \bar{\Omega}_{m}>0} \frac{1}{\tau_{m, x x x}} \psi_{m, x x x}=\psi_{m, w}^{i n c} \sum_{\bar{A}_{x x x} \cdot \bar{\Omega}_{m}>0} \frac{1}{\tau_{m, x x x}}, \quad x x x \in\{f e p, f e z, f p z, p e z\} .
$$

We can use the simplified closures shown in equation (90)to find the following result:

$$
\begin{aligned}
& \psi_{m, w}+\sum_{\bar{A}_{x x} \cdot \bar{\Omega}_{m}>0} \frac{1}{\tau_{m, x x x}}\left[\psi_{m, w}+\beta\left(\tau_{m, x x x}\right)\left(\psi_{m, w}-\psi_{m, w}^{i n c}\right)\right]=\psi_{m, w}^{i n c} \sum_{\bar{A}_{x x x} \cdot \bar{\Omega}_{m}>0} \frac{1}{\tau_{m, x x x}}, \\
& \psi_{m, w}\left[1+\sum_{\bar{A}_{x x x} \cdot \bar{\Omega}_{m}>0} \frac{1+\beta\left(\tau_{m, x x x}\right)}{\tau_{m, x x x}}\right]=\psi_{m, w}^{i n c}\left[\sum_{\bar{A}_{x x x} \cdot \bar{\Omega}_{m}>0} \frac{1+\beta\left(\tau_{m, x x x}\right)}{\tau_{m, x x x}}\right] \\
& \psi_{m, w}=\psi_{m, w}^{i n c} \frac{\vartheta}{1+\vartheta}, \quad \text { where } \quad \vartheta \equiv \sum_{\bar{A}_{x x x} \cdot \bar{\Omega}_{m}>0} \frac{1+\beta\left(\tau_{m, x x x}\right)}{\tau_{m, x x x}} .
\end{aligned}
$$

Using this definition of $\vartheta$ we note that this parameter is always positive since both $\beta_{m, x x x}$ and $\tau_{m, x x x}$ are nonnegative. Now, using equation (95) we can eliminate $\psi_{m, w}$ in the closure equations shown in equation (90).

$$
\left.\begin{array}{ll}
\psi_{m, f e p}=\psi_{m, w}, & \vec{A}_{f e p, w} \cdot \vec{\Omega}_{m}>0, \\
\psi_{m, p e z}=\psi_{m, w}^{i n c} \frac{\vartheta-\beta\left(\tau_{m, p e z}\right)}{1+\vartheta}, & \vec{A}_{p e z, w} \cdot \vec{\Omega}_{m}>0, \\
\psi_{m, f p z}=\psi_{m, w}^{i n c} \frac{\vartheta-\beta\left(\tau_{m, f p z}\right)}{1+\vartheta}, & \vec{A}_{f p z, w} \cdot \vec{\Omega}_{m}>0, \\
\psi_{m, f e z}=\psi_{m, w}^{i n c} \frac{\vartheta-\gamma\left(\tau_{m, f e z}\right)}{1+\vartheta}, & \vec{A}_{f e z, w} \cdot \vec{\Omega}_{m}>0,
\end{array}\right\} \text { for }\left\{\begin{array}{r}
\sigma_{s}=0, \\
Q_{m, c}=0 .
\end{array}\right.
$$


It follows that the exiting surface averaged angular fluxes above will also be non-negative if $\vartheta-\beta\left(\tau_{m, x x x}\right)>0$ and $\vartheta-\gamma\left(\tau_{m, f e z}\right)>0$. Let us examine this expression.

$$
\begin{aligned}
\vartheta-\beta\left(\tau_{m, x x x}\right) & =\sum_{\vec{A}_{y y y} \cdot \bar{\Omega}_{m}>0} \frac{1+\beta\left(\tau_{m, y y y}\right)}{\tau_{m, y y y}}-\beta\left(\tau_{m, x x x}\right) \\
& \geq \frac{1+\beta\left(\tau_{m, x x x}\right)}{\tau_{m, x x x}}-\beta\left(\tau_{m, x x x}\right) \\
& \geq \frac{1+\beta\left(\tau_{m, x x x}\right)-\tau_{m, x x x} \beta\left(\tau_{m, x x x}\right)}{\tau_{m, x x x}}
\end{aligned}
$$

Using the definitions for $\beta\left(\tau_{m, p e z}\right), \beta\left(\tau_{m, f p z}\right)$ and $\chi\left(\tau_{m, f e z}\right)$ in place $\beta\left(\tau_{m, x x x}\right)$ in equation (7) we find that we meet this criteria and our scheme will always be non-negative in a source free purely absorbing media when the incident angular fluxes are also non-negative.

$$
\begin{aligned}
& \left.\frac{1+\beta\left(\tau_{x x x}\right)-\tau_{x x x} \beta\left(\tau_{m, x x x}\right)}{\tau_{x x x}}\right|_{\substack{p e z z \\
f f z}}=\frac{36-12 \tau_{x x x}+\tau_{x x x}^{2}}{\tau_{x x x}\left(18+6 \tau_{x x x}+\tau_{x x x}^{2}\right)} \geq 0 \\
& \left.\frac{1+\gamma\left(\tau_{x x x}\right)-\tau_{x x x} \gamma\left(\tau_{m, x x x}\right)}{\tau_{x x x}}\right|_{f e z}=\frac{3+3 \tau_{m, f e z}-2 \tau_{m, f e z}^{2}+4 \tau_{m, f e z}^{3}}{2 \tau_{m, f e z}^{2}\left(1+\tau_{m, f e z}+2 \tau_{m, f e z}^{2}\right)}>0
\end{aligned}
$$

In this section, we have shown that our method will preserve a non-negative solution in a source-free, purely absorbing media when the incident information is also non-negative. This is an important result because it tells us that our method is resistant to oscillations in the solution that some spatial discretizations suffer.

\section{E. Operator Symmetry}

In general, both the analytic and discrete transport operators are asymmetric. The transport operator is only symmetric in the special case where all scattering is isotropic. Because our discretized system of equations is generally asymmetric it is normally solved using source-iteration (i.e. Richardson iteration). Many standard iterative techniques (e.g. conjugate gradients) can not be used to solve the system directly because of its asymmetry. Accelerating preconditioners such as diffusion synthetic acceleration, DSA, are currently favored over the use of more complex iterative solvers that can handle asymmetric systems for speeding the iterative convergence rate.

As discussed above, the 3D UCBL spatial discretization developed in this paper is asymmetric because the underlying analytic transport operator is asymmetric. We are also interested in the symmetry of the diffusion discretization that it captures in the thick diffusion limit. This property is important to understand so that perhaps some efficient DSA preconditioner may be employed to accelerate the convergence rate of our iterative solver.

From Section III.B. we know that to leading order our spatial discretization limits to Palmer's discrete diffusion discretization in the thick diffusion limit. Thus, we should be able to use his solver to accelerate 
our transport scheme. Palmer states that his diffusion discretization is asymmetric but does not demonstrate this property. ${ }^{9}$ To verify his statement we demonstrate the asymmetry of the leading-order solution below. In the thick diffusion limit, the leading-order operator (i.e. the diffusion operator) is asymmetric if there are entries in the diffusion operator matrix that are not equal to their transposed counterparts. Thus, if we can demonstrate that there is an entry in this matrix that is not equal to its counterpart for any single problem we will have demonstrated that this diffusion discretization is asymmetric.

We start by writing Palmer's discrete diffusion equation in matrix notation. This equation is equivalent to the leading-order balance equation (40) where the wedge face currents have been replaced by their leading-order scalar flux representation.

$$
\mathbf{M} \vec{\phi}^{(0)}=\mathbf{S}
$$

Once we construct $\mathbf{M}$ we only need to show that there exists one entry in $\mathbf{M}$ where $\mathbf{M}_{p p^{\prime}} \neq \mathbf{M}_{p^{\prime} p}$. If this inequality holds true for just one case then our operator, in the general case, is asymmetric. To build $\mathbf{M}$ we consider the grid shown in Figure 7. This grid consists of eight zones. Six of these zones are perfect cubes. The other two zones are seven sided polyhedra. The second grid at the lower right in this figure shows the back half of our problem subdivided into corners. Additionally, the two corners of primary interest are also broken into wedges. We have also labeled a few points and wedges that are of primary interest in this analysis.

We start by considering the $p^{\text {th }}$ row of $\mathbf{M}$. This row is the diffusion balance equation for the dual cell volume formed by all of the corners that intersect the vertex $p$.

$$
\mathbf{M}_{p} \vec{\phi}^{(0)}=\sum_{w \in p} \vec{A}_{f e z, w} \cdot J_{f e z, w}^{(1)}+\sum_{c \in p} \sigma_{t, c} V_{c} \phi_{c}^{(0)}=\sum_{c \in p} V_{c} Q_{c}^{(0)}=\mathbf{S}_{p} .
$$

We use equation (18) to express the $f e z$ current in terms of the leading-order scalar flux. Note that equation (18) can be rewritten in the simpler form shown in equation (14) that is used for this analysis.

$$
\vec{A}_{f e z, w} \cdot J_{f e z, w}^{(1)}=\frac{1}{9 \sigma_{t} V_{w}} \vec{A}_{f e z, w} \cdot\left(\vec{A}_{f e z, w} \phi_{c}^{(0)}+\vec{A}_{f e p, w} \phi_{z}^{(0)}+\vec{A}_{f p z, w} \phi_{e}^{(0)}+\vec{A}_{p e z, w} \phi_{f}^{(0)}\right) .
$$

The zone, edge and face scalar fluxes are defined in terms of the corner scalar fluxes according to equations (15), (16) and (18). Since we are only interested in how $\phi_{p}^{(0)}$ depends on $\phi_{p^{\prime}}^{(0)}$ (i.e. the $\left(p, p^{\prime}\right)$ entry of $\mathbf{M}$ ) we need to compile only the pieces of (101) that contain $\phi_{p^{\prime}}^{(0)}$. We use "evaluated at" nomenclature to represent our terms of interest.

$$
\mathbf{M}_{p p^{\prime}} \phi_{p^{\prime}}^{(0)}=\left[\sum_{w \in p} \vec{A}_{f e z, w} \cdot J_{f e z, w}^{(1)}+\sum_{c \in p} \sigma_{t, c} V_{c} \phi_{c}^{(0)}\right]_{\phi_{p^{\prime}}^{(0)}} .
$$




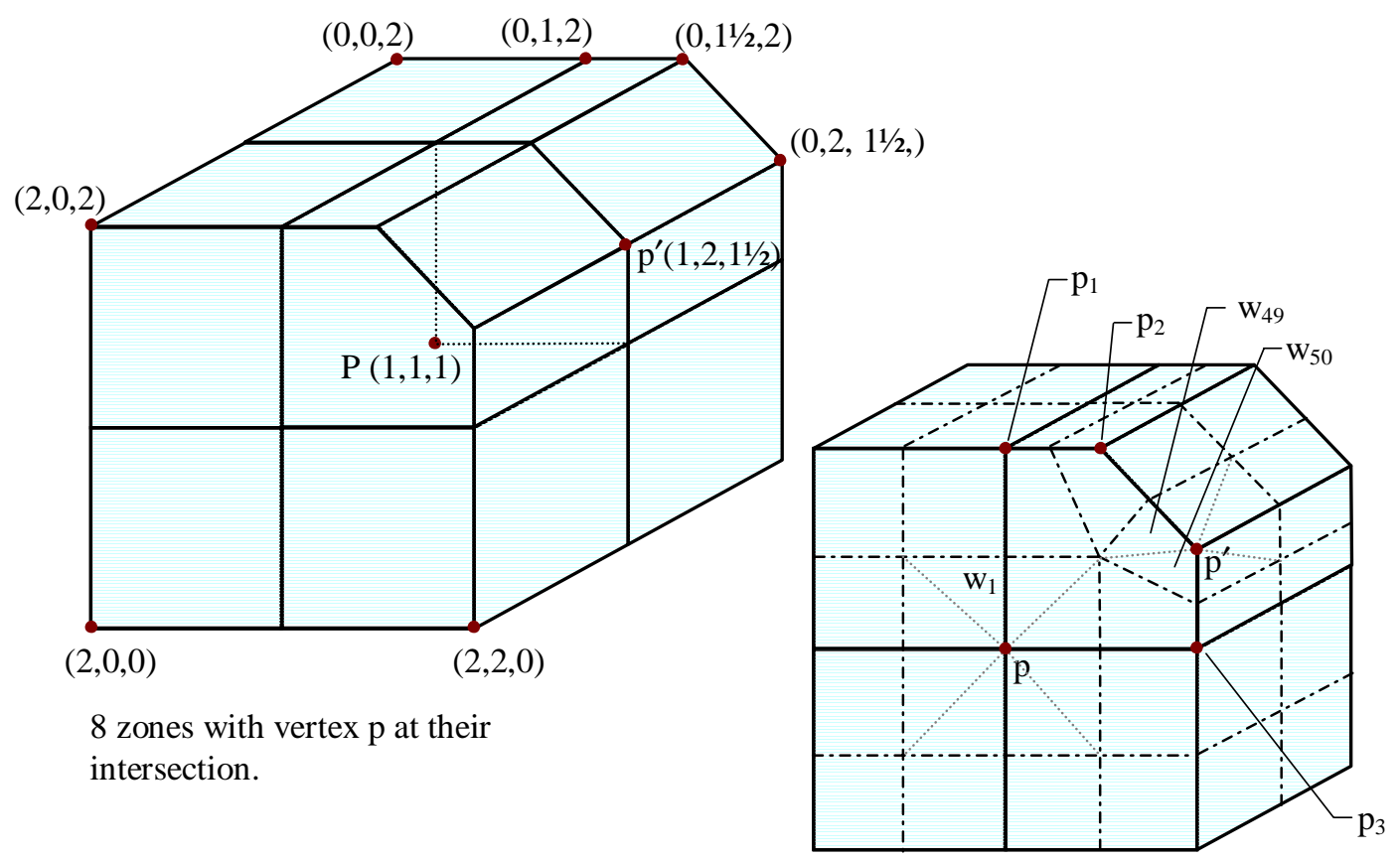

The back 4 zones sectioned into corners. The corners near $\mathrm{p}$ and $\mathrm{p}^{\prime}$ are sectioned into wedges

Figure 7. An eight-cell grid used to demonstrate the asymmetry of our diffusion operator.

Now we examine equation (102) for wedge $w_{1}$ (see Figure 7). We initially note that $\vec{A}_{f e z, w} \perp \vec{A}_{f e p, w}$ and $\vec{A}_{f e z, w} \perp \vec{A}_{p e z, w}$ so that equation (101) only depends on the corner and the edge scalar fluxes. We further note that neither the corner nor the edge flux depends on $\phi_{p^{\prime}}^{(0)}$. The absorption term for the corner containing $w_{1}$ does not depend on $\phi_{p^{\prime}}^{(0)}$ either, thus the contribution to $\mathbf{M}_{p p^{\prime}}$ from wedge $w_{1}$ is zero. A cursory examination of the remaining 47 wedges that surround point $p$ will reveal that $\phi_{p}^{(0)}$ does not depend on $\phi_{p^{\prime}}^{(0)}$ so that the $\left(p, p^{\prime}\right)$ entry of matrix $\mathbf{M}$ is zero for this grid.

$$
\mathbf{M}_{p p^{\prime}}=0 .
$$

The leading-order scalar flux $\phi_{p}^{(0)}$ does not depend on $\phi_{p^{\prime}}^{(0)}$ because of the orthogonal nature of the corners surrounding the vertex $p$.

We can perform a similar analysis to see how the solution $\phi_{p^{\prime}}^{(0)}$ depends on $\phi_{p}^{(0)}$ (i.e. the value of $\mathbf{M}_{p^{\prime}}$ ). We start by examining the balance equation for wedge $w_{49}$ (see Figure 7). We note that for this cell we have only $\vec{A}_{f e z, w} \perp \vec{A}_{f e p, w}$ so that the contribution to our balance equation from $w_{49}$ has the following form. 


$$
\begin{aligned}
& \left.\vec{A}_{f e z, w} \cdot J_{f e z, w}^{(1)}\right|_{\phi_{p}^{(0)}, w 49}=\left.\frac{1}{9 \sigma_{t} V_{w}} \vec{A}_{f e z, w} \cdot\left(\vec{A}_{f e z, w} \phi_{c}^{(0)}+\vec{A}_{f p z, w} \phi_{e}^{(0)}+\vec{A}_{p e z, w} \phi_{f}^{(0)}\right)\right|_{\phi_{p}^{(0)}}, \\
& =\frac{1}{9 \sigma_{t} V_{w}} \vec{A}_{f e z, w} \cdot\left[\begin{array}{l}
\vec{A}_{f e z, w} \phi_{p^{\prime}}^{(0)}+\frac{1}{2} \vec{A}_{f p z, w}\left(\phi_{p^{\prime}}^{(0)}+\phi_{p 1}^{(0)}\right) \\
+\frac{1}{5} \vec{A}_{p e z, w}\left(\phi_{p^{\prime}}^{(0)}+\phi_{p}^{(0)}+\phi_{p 1}^{(0)}+\phi_{p 2}^{(0)}+\phi_{p 3}^{(0)}\right)
\end{array}\right]_{\phi_{p}^{(0)}}, \\
& =\frac{1}{45 \sigma_{t} V_{w}}\left(\vec{A}_{f e z, w} \cdot \vec{A}_{p e z, w}\right) \phi_{p}^{(0)} .
\end{aligned}
$$

We have replaced the edge and face leading-order scalar fluxes with their definitions in terms of vertex centered quantities (see equations (16) and (17)).

Because of the geometry chosen in Figure 7 only three additional wedges contribute to $\mathbf{M}_{p^{\prime} p}$. These wedges are $w_{50}$ (which shares the $f p z$ face with $w_{49}$ ) and wedges $w_{73}$ and $w_{74}$. Wedges $w_{73}$ and $w_{74}$ are the counterparts to wedges $w_{49}$ and $w_{50}$, which live in the front half of the grid shown in Figure 7. These are the only wedges that have a cell face that intersects point $p$. For more complex geometries the zone midpoint scalar flux would come into play linking more wedges to point $p$. However, in our simple case the solution at point $p^{\prime}$ interacts with the solution at point $p$ only via this face-centered value.

Examining the net current for the remaining three wedges that can contribute to $\mathbf{M}_{p^{\prime} p}$ we find:

$$
\left.\vec{A}_{f e z, w} \cdot J_{f e z, w}^{(1)}\right|_{\phi_{p}^{(0)}, w}=\frac{1}{45 \sigma_{t} V_{w}}\left(\vec{A}_{f e z, w} \cdot \vec{A}_{p e z, w}\right) \phi_{p}^{(0)}, \quad \text { for } w=w_{50}, w_{73} \text { and } w_{74} .
$$

This means that $\mathbf{M}_{p^{\prime} p}$ has a non-zero value for the grid shown in Figure 7.

$$
\mathbf{M}_{p^{\prime} p}=\sum_{w \in\left\{w_{49}, w_{50}, w_{73}, w_{74}\right\}} \frac{1}{45 \sigma_{t} V_{w}}\left(\vec{A}_{f e z, w} \cdot \vec{A}_{p e z, w}\right) \neq \mathbf{M}_{p p^{\prime}}=0 .
$$

In this example we have demonstrated the asymmetry of the discrete diffusion operator that the $3 \mathrm{D}$ UCBL discretization limits to in the thick diffusion limit. For a purely orthogonal mesh this operator is actually symmetric but for general polyhedral grids it will be, in general, asymmetric. That is, the discrete diffusion solution at vertex $p$ will depend on the solution at $p^{\prime}$ in a different way than the solution at vertex $p^{\prime}$ depends on the solution at $p$.

As we mentioned before, this property is not ideal but it does not degrade the quality of the solution. The asymmetry of the diffusion operator simply limits the types of iterative solvers that may be employed to accelerate the convergence process. This is a concern because iterative solution algorithms for asymmetric systems are typically not as robust or fast as those used for symmetric systems.

\section{F. Minimize Spreading of a Beam}

Our discretization scheme has also been designed to minimize the spreading of a beam in a purely absorbing media. In many numerical schemes (including 2D upstream corner balance) the spatial discretization will artificially diffuse a beam. ${ }^{14} \mathrm{We}$ are able to limit this non-physical grid induced diffusion in 3D UCBL by using wedge-face area projection-weighting terms (see equation (56)). In most spatial discretization schemes, incident information is used to compute a cell average in a similar manner to what 
we have shown for 3D UCBL. However, because we use sub-cell volumes we can track information on a finer scale inside of each cell so that we limit the propagation of the angular flux onto cell surfaces that are not physically downstream of the incident information. This procedure limits the artificial diffusion caused by the spatial discretization.

Figure 8 shows a cartoon of a single zone with an incident beam on one face. The cell on the left spreads the beam onto both exiting faces. The cell on the right has been broken in the $2 \mathrm{D}$ analog of wedge sub-cell volumetric elements. Because we have internal boundaries we are able to restrict the outflow to the right surface. In fact, the solution in the upper left portion of the cell remains unaffected by the incident beam.
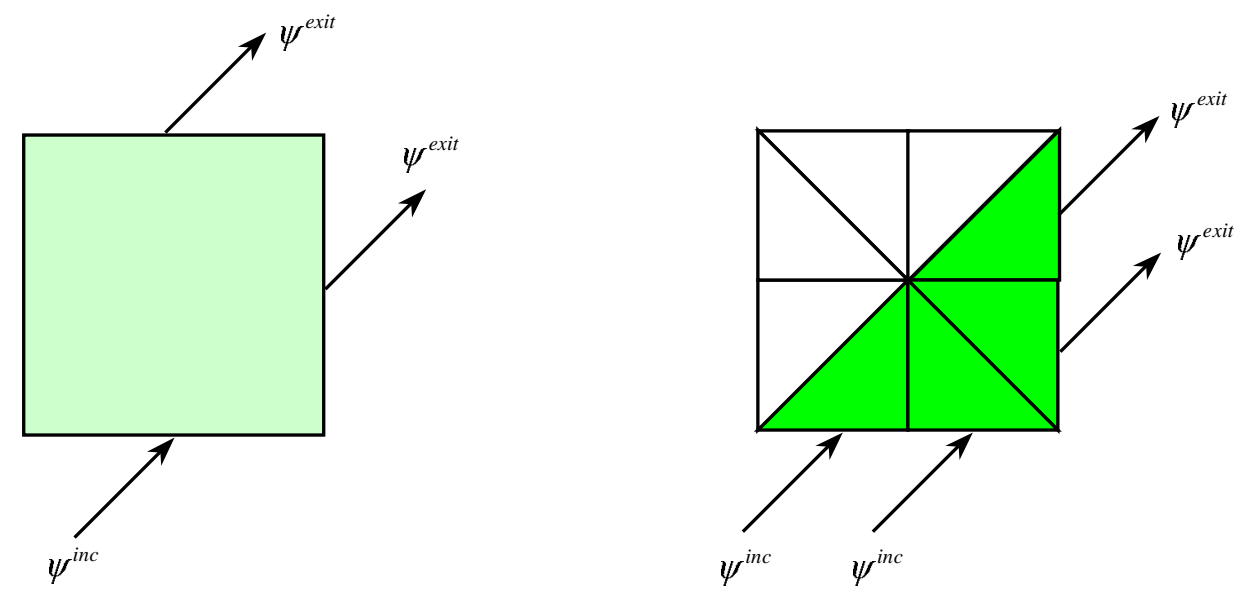

Figure 8. This example shows how UCBL limits the spreading of beams.

The example shown in this figure is an idealized case in which the wedge boundary lines up exactly with the quadrature direction. If the cell in this figure were wider than the one shown, then the wedges in the upper left of the cell would have had non-zero angular fluxes. However, because we depend on the magnitude of the projected area of each wedge to determine the fraction of the angular flux that crosses that face we limit the artificial diffusion that is seen in other spatial discretization schemes.

We have run two test problems that are the analog of the above cartoon. The first problem is a $8 \mathrm{~cm}^{3}$ cube with $10^{3}$ cells $\left(20^{3}\right.$ corners) that has a mono-directional beam incident on the right face. This beam is oriented to point left, up and out of the page. We have vacuum boundary conditions and a vacuum material $\left(\sigma_{t}=0, \sigma_{s}=0, \sigma_{f}=0\right)$. A 3D plot of the corner-averaged scalar flux is shown in Figure 9. We are running an $S_{2}$ quadrature set and all of the cells are exact cubes so the direction of interest lines up exactly with the wedge boundaries. In this special case there is no numerical diffusion of the beam. The front of the problem is fully illuminated by the incident beam and the back half of the problem remains dark. The line of corners that separate the illuminated and dark corners have a corner-averaged scalar flux that is one half the intensity of the fully illuminated corners. In these corners half of the wedges are illuminated and half are 
not. The solution is a step function on the wedges but not on corners. The corners that form a diagonal between the back lower right and the front upper right have a scalar flux value that is a third of the fully illuminated corners. For this line of corners only one third of the wedges in each corner are illuminated.
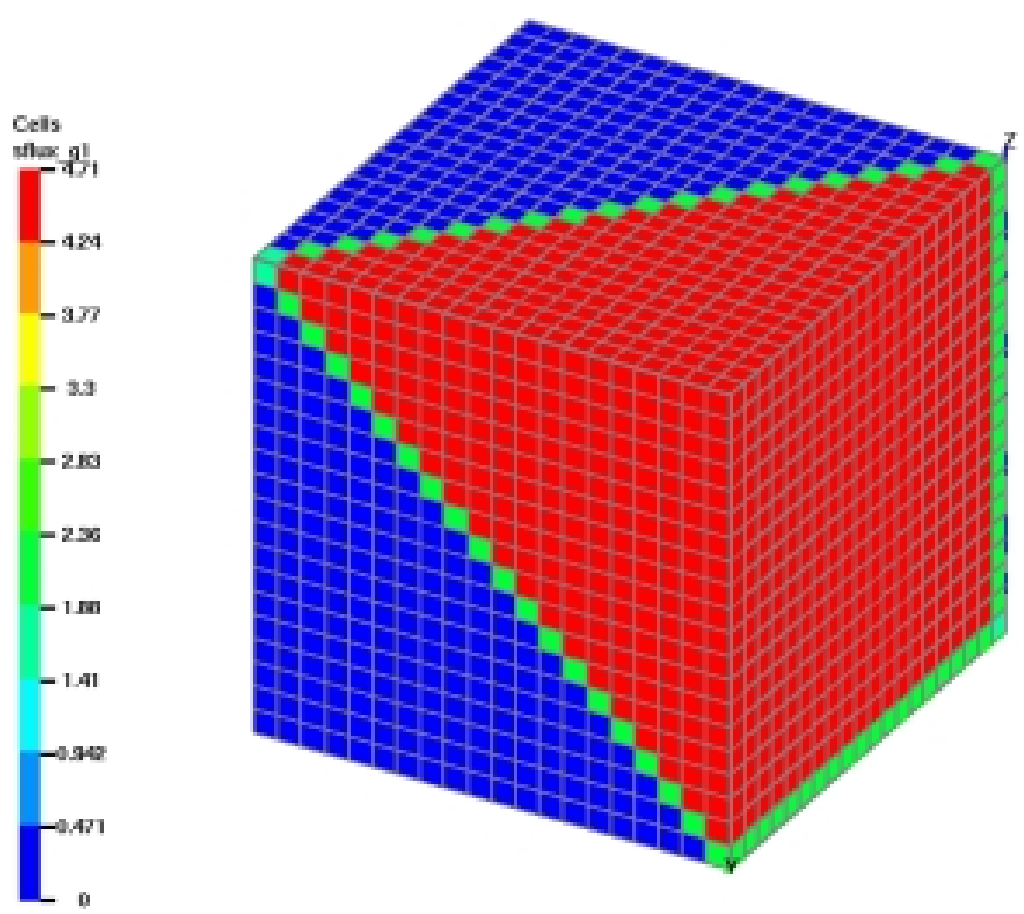

Figure 9. Numerical test that demonstrates that 3D UCBL captures exactly the analytic solution for idealized problems. 
Figure 10 shows a plot of the scalar flux as a function of the $\mathrm{x}$-dimension. We examine the corneraveraged scalar fluxes for the row of corners whose midpoints are at $\bar{y}=\bar{z}=1.05 \mathrm{~cm}$. This one-dimensional lineout shows how the 3D UCBL solution captures exactly the analytic step function. The scalar flux at $x=1.05 \mathrm{~cm}$ is one third of the maximum value since only two of the six wedges in this corner are illuminated. We have also plotted a 3D Linear Discontinuous, LD, solution for comparison against UCBL. The LD solution also captures the one-third illuminated cell exactly. However, the LD solution overshoots and undershoots the step function in the reference solution reporting negative flux values to the right of the step function. The LD solution was computed using the transport code PARTISN. ${ }^{22}$

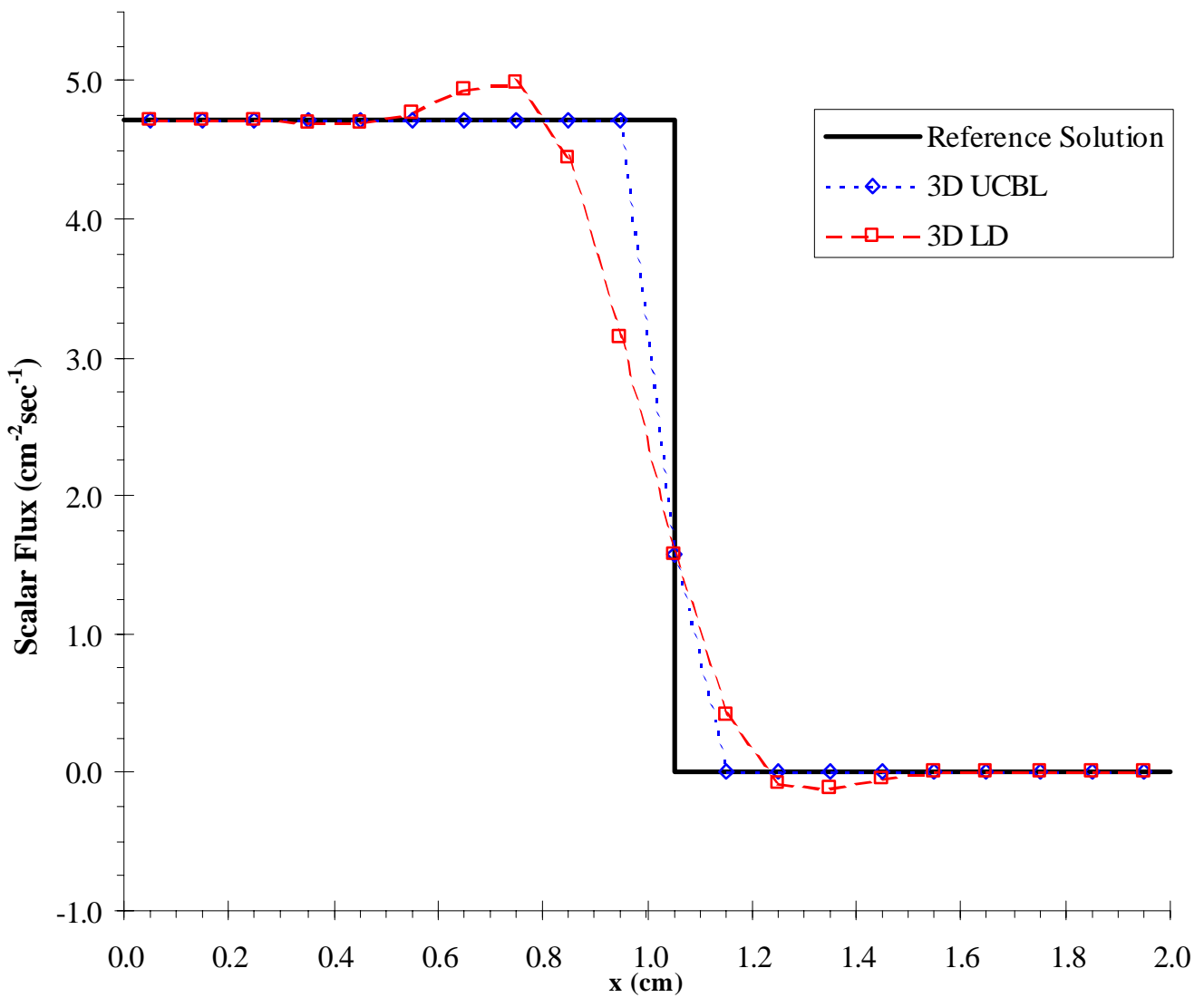

Figure 10. Lineout solution for a beam in a vacuum (ideal geometry). 
The second problem we look at is very similar to the first but we have refined the spatial grid in the $y$ and $z$-directions. The overall size of the problem is still $8 \mathrm{~cm}^{3}$. But there are now 32 corners in the $y$-and $z$-directions. The material is still a vacuum and we use a $S_{2}$ quadrature set. Since the corner volumes are no longer perfect cubes 3D UCBL does not capture the analytic solution exactly. This smearing of the step function solution can be seen in Figure 11. It takes several corners for the solution to drop from full illumination to no illumination. Another feature seen in the plot is that there are two rows of corners on the right face that are not fully illuminated. This is expected because the corners are longer in the $x$-direction than in the other two directions. From simple geometric considerations we notice that the first row of corners is less than half illuminated and the second row is more than half illuminated but still not fully illuminated.

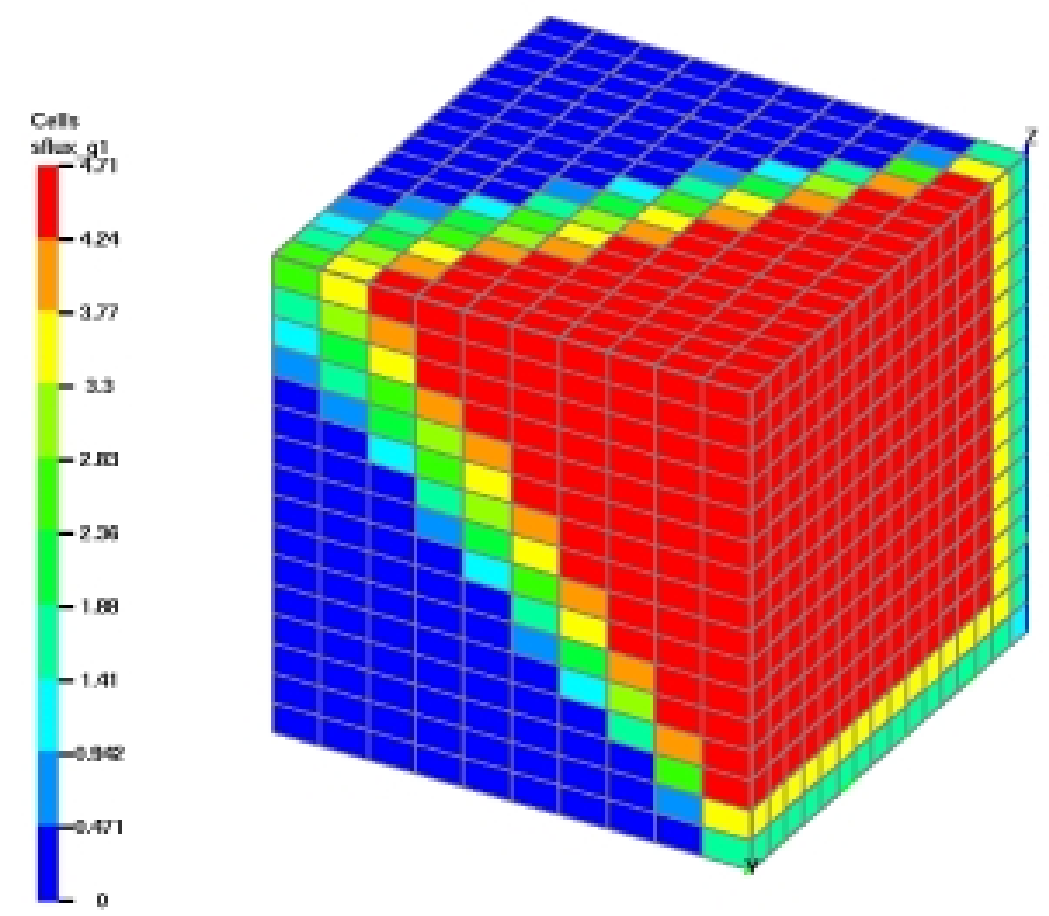

Figure 11. Numerical test that demonstrates that 3D UCBL limits the numerical diffusion of a beam in vacuum for general problems.

Once again it is useful to examine a lineout through the problem to show how close the solution is to the analytic step function. In Figure 12 we plot the scalar flux as a function of $x$ through the columns of cells whose midpoints are at $\bar{y}=\bar{z}=1.03125 \mathrm{~cm}$. In the plot we also show the analytic solution and a 3D LD solution for reference. Both numerical solutions do a good job of representing the step function. The LD solution looks very similar to the solution shown in Figure 10. It overshoots the solution before the step 
drop and undershoots the solution after the step. The LD solution produces negative solutions when it undershoots the analytic solution. As we mentioned in Section III.D. the 3D UCBL solution will remain strictly non-negative. The UCBL solution also appears to capture a monotonic approximation to the step. The trade off made for positivity and monotonicity is that the 3D UCBL solution is a less accurate approximation to the analytic solution.

Overall 3D UCBL does a good job of limiting beam spread in a vacuum. It can actually exactly capture the analytic solution in special cases. The solution captured by 3D UCBL is somewhat less accurate when compared to 3D LD but does remain purely positive and the solution appears to be monotonic. Like LD, it numerically smears the step function over several cells. This is normal for most numerical approximations. Additionally, the performance of 3D UCBL is significantly better than UCB in 2D where numerical diffusion spreads the beam to all cells in the problem.

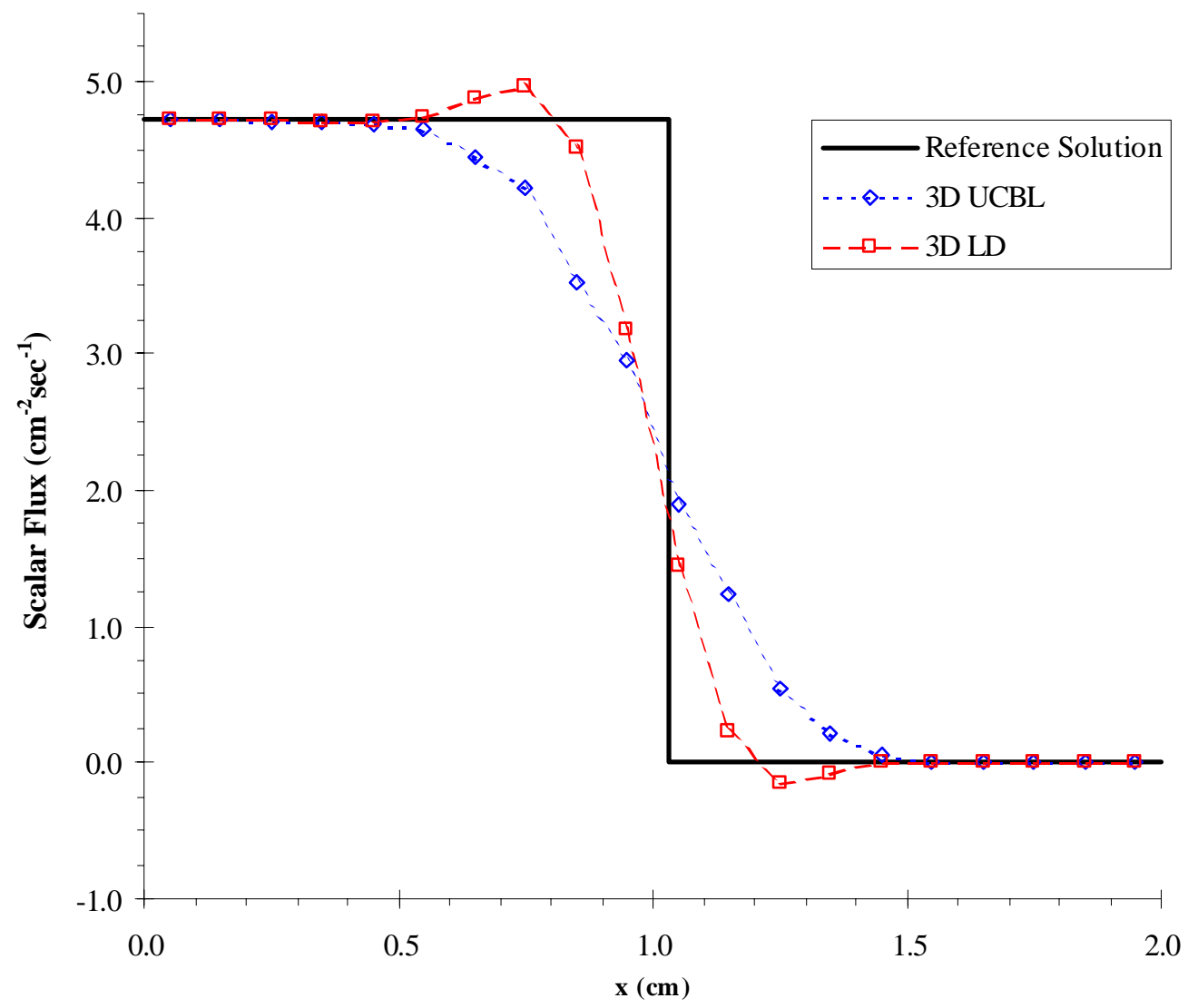

Figure 12. Lineout solution for a beam in a vacuum (general geometry). 


\section{G. Truncation Error Study}

The order of the local truncation error is a measure of the error in the solution introduced by the spatial discretization. We will not complete a formal analysis to find the local truncation error of 3D UCBL. However, we will perform a series of numerical tests that will reveal the order of the local truncation error.

Recall that in Section II. G. we refined two of our closure equations to perform better in source free purely scattering media. This development was implemented to improve the order of the local truncation error - but only for slab-like problems. In this section we examine the performance (i.e. validity) of these additions. For slab-like problems we expect that UCBL will capture the desired $2^{\text {nd }}$-order truncation error. However, it is not clear how our scheme will perform in truly 3D problems.

Consider a 3D rectangular problem consisting of brick-shaped cells. We assume a source-free medium which is 2x20x20 mean-free paths thick and which has an isotropic incident intensity on the minimum $x$-face. The removal cross section is half of the total. There are reflective boundary conditions on the top, bottom, left and right faces to simulate a slab problem. An $\mathrm{S}_{4}$ level-symmetric quadrature set is used. We will solve this problem using 3D UCBL and compare against the diamond differencing (DD) and linear discontinuous (LD) spatial discretizations. Solutions for the later two spatial discretizations were obtained from the PARTISN code package. Below in Table I we report the exiting flow rate (EFR) from the maximum $x$-face and the total absorption rate (AR) for various mesh spacings. The errors listed in this table were generated by assuming that a $128 \times 128 \times 128$ 3D LD problem obtains the correct solution. The error reduction ration (ERR) is reported for EFR errors.

TABLE I

Errors in Exiting Flow Rate (EFR) and Absorption Rate (AR) and the Error Reduction

Ratio (ERR) for Various Methods and Grids for a Slab Like Problem

\begin{tabular}{|c|ccc|ccc|ccc|}
\hline \multirow{2}{*}{$\begin{array}{c}\text { Number } \\
\text { of cells }\end{array}$} & \multicolumn{9}{|c|}{ Method } \\
\cline { 2 - 11 } & \multicolumn{3}{|c|}{ DD error } & \multicolumn{3}{c|}{ LD error } & \multicolumn{3}{c|}{ 3D UCBL error } \\
\cline { 2 - 11 } & AR & EFR & ERR & \multicolumn{1}{|c|}{ AR } & EFR & ERR & AR & EFR & ERR \\
\hline $2 \times 4 \times 4$ & \multicolumn{3}{|c}{ N/A N/A } & \multicolumn{2}{|c}{ N/A } & N/A & 7.947 & 6.530 & N/A \\
$4 \times 4 \times 4$ & 6.646 & 6.839 & N/A & 1.511 & 0.641 & N/A & 2.469 & 1.664 & 3.9 \\
$8 \times 4 \times 4$ & 1.669 & 1.719 & 4.0 & 0.218 & 0.087 & 7.4 & 0.665 & 0.423 & 3.9 \\
$16 \times 4 \times 4$ & 0.330 & 0.431 & 4.0 & 0.029 & 0.012 & 7.3 & 0.172 & 0.107 & 4.0 \\
$32 \times 4 \times 4$ & 0.104 & 0.108 & 4.0 & 0.006 & 0.002 & 6.0 & 0.044 & 0.027 & 4.0 \\
$64 \times 4 \times 4$ & 0.026 & 0.029 & 3.7 & 0.0 & N/A & N/A & 0.012 & 0.007 & 3.9 \\
\hline
\end{tabular}


The reduction in error that is observed by doubling the number of cells in the $x$-direction is about 4 for DD, about 8 for LD and about 4 for 3D UCBL. We know that LD is $3^{\text {rd }}$-order accurate, and that DD is $2^{\text {nd }}$-order accurate for slab problems and it would appear from this problem that $3 \mathrm{D}$ UCBL is also $2^{\text {nd }}$-order accurate.

If we run the same set of problems but refine the mesh in all dimensions then our cells will appear 3D instead of slab-like. These results are shown in Table II. There are several additional points worth noting. First, both LD and DD will obtain the same solution to this problem that their slab-geometry version would obtain (given the same quadrature set). This solution is independent of the number of cells along the $y$ and $z$ axes (where the solution is constant). The same is not true of the wedge-based UCBL scheme. It does not obtain the slab-geometry UCBL solution except in the limit of large cell dimensions in the $y$ and $z$ dimensions. As one consequence, this means that the $32 \times 32 \times 32$ UCBL solution shown in Table II is less accurate than the $32 \times 4 \times 4$ solution shown above. The 3D UCBL results presented in this table are not conclusive because the mesh is not fine enough to obtain a clear picture of the ERR. The large computational resources required to examine more refined meshes restricted us from obtaining such results. However, from these results it appears that 3D UCBL is not, in general, a second-order scheme even though the ERR appears to be fairly large for coarse grids. We will revisit this question in the next chapter where we will demonstrate that $3 \mathrm{D}$ UCBL is a first-order accurate scheme for general 3D meshes.

TABLE II

Errors in Exiting Flow Rate (EFR) and Absorption Rate (AR) and the Error Reduction Ratio (ERR) for Various Methods and Grids for a 3D Problem

\begin{tabular}{|c|c|c|c|c|c|c|c|c|c|}
\hline \multirow{3}{*}{$\begin{array}{l}\text { Number } \\
\text { of cells }\end{array}$} & \multicolumn{9}{|c|}{ Method } \\
\hline & \multicolumn{3}{|c|}{ DD error } & \multicolumn{3}{|c|}{ LD error } & \multicolumn{3}{|c|}{ 3D UCBL error } \\
\hline & AR & EFR & ERR & AR & EFR & ERR & $\mathrm{AR}$ & EFR & ERR \\
\hline $2 \times 2 \times 2$ & \multicolumn{2}{|c|}{ N/A } & N/A & \multicolumn{2}{|c|}{ N/A } & N/A & 5.294 & 11.102 & N/A \\
\hline $4 \times 4 \times 4$ & 6.646 & 6.839 & N/A & 1.511 & 0.641 & N/A & 1.726 & 3.370 & 3.3 \\
\hline $8 \times 8 \times 8$ & 1.669 & 1.719 & 4.0 & 0.218 & 0.087 & 7.4 & 0.539 & 1.034 & 3.3 \\
\hline $16 \times 16 \times 16$ & 0.330 & 0.431 & 4.0 & 0.029 & 0.012 & 7.3 & 0.181 & 0.632 & 1.6 \\
\hline $32 \times 32 \times 32$ & 0.104 & 0.108 & 4.0 & 0.006 & 0.002 & 6.0 & 0.065 & 0.154 & 4.1 \\
\hline $64 \times 64 \times 64$ & 0.026 & 0.029 & 3.7 & 0.0 & N/A & N/A & & & N/A \\
\hline
\end{tabular}

There are two forces at work to limit 3D UCBL to $1^{\text {st }}$-order accuracy. First, we only designed the closure equations to capture second-order accuracy for slab-like problems. Tuning our closure equations to capture a more accurate representation of the single-cell attenuation factor did this. That is, our development only considered a direction that did not cross multiple boundaries of one corner. In a general 3D problem our approximation of the single-cell attenuation factor will not be this accurate because we will 
be crossing corner surfaces that live in the other two planes. We can only tune our closure equations to get this accurate representation of the single cell attenuation factor for slab-like problems. Thus, we are limited to first order accuracy for general problems.

The above argument is actually the manifestation of a more serious limitation. Godunov has shown that for the transport equation a spatial discretization scheme will be limited to $1^{\text {st }}$-order accuracy if it is an explicit two-layer scheme that is both algebraically linear and monotonic. ${ }^{23} 3 \mathrm{D}$ UCBL is certainly a linear discretization scheme. Additionally, our system of equations is an explicit two-layer scheme because only previously calculated ("upstream") information from nearest neighbors is used to calculate the primary unknown for the current wedge. We have also shown that it is a purely positive and monotonic scheme (at least for a subset of problems). Because we meet all of these criteria Godunov's theorem confirms that we will be limited to $1^{\text {st }}$-order accuracy. It should be noted that $2 \mathrm{D}$ UCB is not limited by this theorem because it is not a monotonic scheme. In fact, $2 \mathrm{D} \mathrm{UCB}$ is $2^{\text {nd }}$-order accurate.

\section{H. Summary of Analysis}

In this chapter we have reviewed all of the properties of our spatial discretization scheme that we initially were interested in. We have successfully devised a wedge-based upstream corner-balance linear (UCBL) method for three-dimensional grids of arbitrary polyhedra. This scheme satisfies robust and accurate equations in the thick diffusion limit, capturing linear solutions regardless of the grid. It has strict particle conservation and can use fully upstream transport sweeps, with no matrix inversions. It is algebraically linear yet stays non-negative in a source-free pure absorber.

Our wedge-based UCB scheme is not without drawbacks. Given a thick diffusive problem with a boundary layer that is not resolved by the grid, this method, with its "Marshak" boundary condition, runs the risk of being in error by up to a factor of two in the worst case. The method appears to be only $1^{\text {st }}$-order accurate; we do not believe it is possible to make it higher order as long as the only information transferred at wedge surfaces is the surface-averaged flux. 


\section{CHAPTER IV}

\section{NUMERICAL RESULTS}

In Chapter II we developed the defining equation of the 3D UCBL spatial discretization. This polyhedral-based spatial discretization was designed from the beginning to have good properties in the thick diffusive limit and reasonable properties in the fine and intermediate limits. In Chapter III we verified many of these properties by completing a full diffusion-limit analysis along with several other analyses that predicted the performance of our scheme in thin problems (positivity in a vacuum, truncation error, unphysical discretization-enhanced diffusion and others). In this chapter we present numerical results for several test problems that will demonstrate some of these features. We examine the performance of 3D UCBL in different material regimes and compare these results to either analytic or numeric benchmark calculations.

In this chapter we will study $\mathrm{k}$ - and alpha-eigenvalue problems, multi-group and multi-region problems and problems with anisotropic scattering. We will examine how robust UCBL is by running a coarsely discretized mesh on a problem that has steep boundary layers between materials and we will revisit the truncation analysis. These tests in conjunction with the numerical results shown in Chapter III will identify strong and weak areas of our discretization. Additionally, we will analyze an unstructured mesh of embedded cylinders that resembles the geometry of a well-logging tool. This simulation will utilize a mesh that is regular in the z-direction so that we can compare the UCBL solution against the solution obtained by Richardson's SMaRT-Z corner balance discretization. This will allow us to establish that the solutions obtained by our scheme compare favorably to existing schemes. Finally, we will examine a large and complex real-life problem. We will simulate a MOX fuel assembly in the Advanced Test Reactor. The one regime that we are unable to test is on grids with mixtures of many different kinds of polyhedra. We were unable to locate a simple grid generator that could produce such complex grids without linking our algorithms into complex AMR hydrodynamic/radiation codes. Thus, all of the problems shown in this section have grids composed of degenerate hexahedral cells even though our scheme is in no way limited to these simple cell shapes. 


\section{A. Infinite K-Eigenvalue Problem}

Our first test problem is an infinite medium k-eigenvalue problem. We have constructed a spherical pie mesh consisting of 20 slices of tetrahedral, pyramidal, prism and hexahedral cells. Each slice contains 10 concentric layers of 10 cells as shown in Figure 13. We used a two-group cross section set with isotropic scattering and no up-scattering.

$$
\begin{aligned}
\sigma_{t} & =\left[\begin{array}{l}
0.7098403 \\
1.5291015
\end{array}\right], \quad \sigma_{s}=\left[\begin{array}{cc}
0.4602541 & 0.0 \\
0.1430985 & 0.9342397
\end{array}\right], \\
v \sigma_{f} & =\left[\begin{array}{l}
0.3761562 \\
1.0361502
\end{array}\right], \quad \chi=\left[\begin{array}{l}
0.3534469 \\
0.6465531
\end{array}\right] .
\end{aligned}
$$

Enforcing reflective boundary conditions on the entire surface of the sphere simulated an infinite medium. The reflective boundary algorithm used in our work is developed in Appendix B. It should be noted that our 3D scheme uses an XYZ coordinate set and not a spherical set. This means that we are testing our algorithm on an unstructured mesh where very few cell boundaries are perpendicular to the cardinal axes.
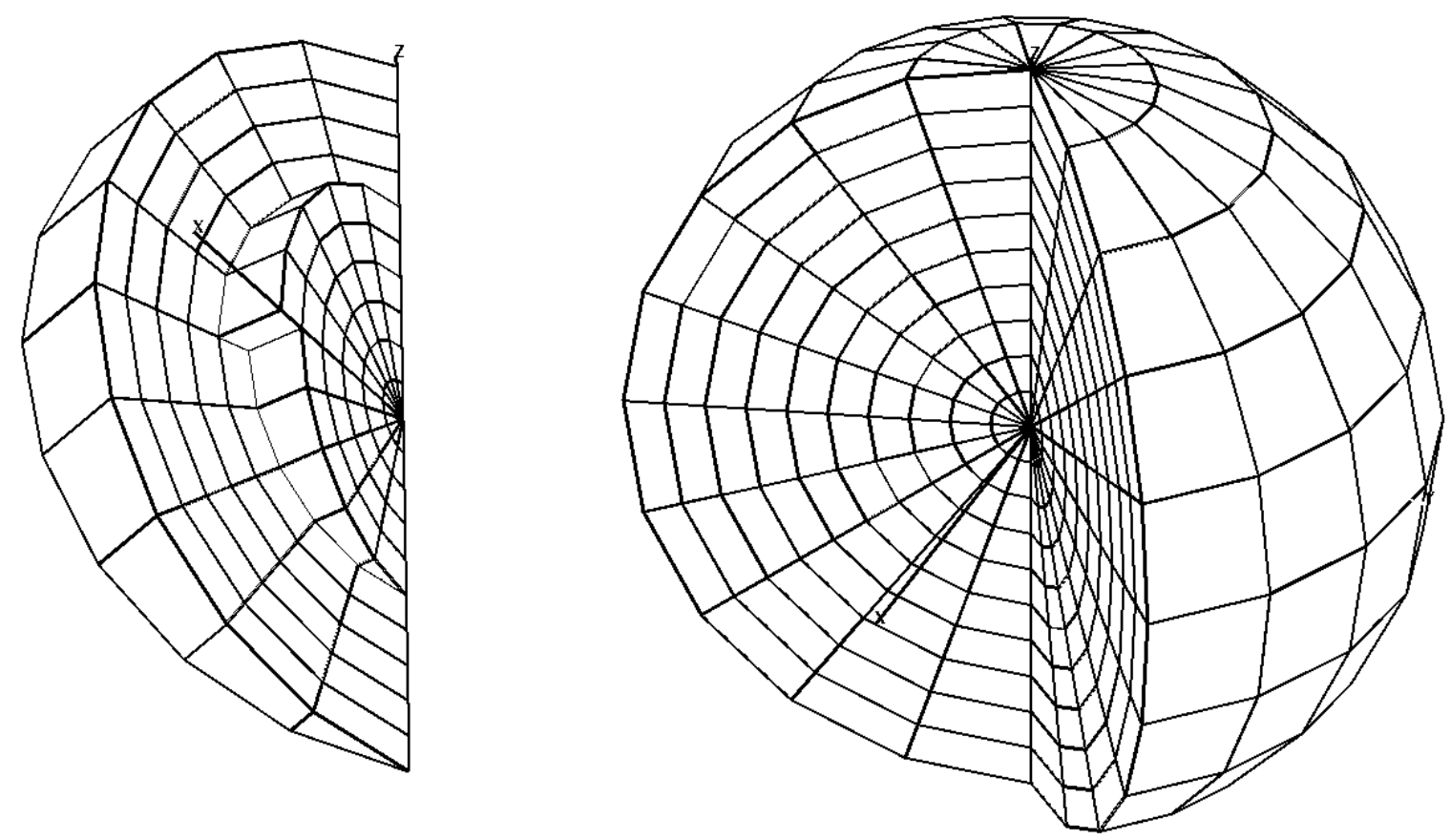

Figure 13. Spherical pie mesh. 
We have chosen to examine an infinite-medium problem because it is relatively easy to derive an analytic expression for the k-eigenvalue. The analytic solution can be derived from equation (4) where we replace the average number of neutrons per fission, $v$, with $v / k$ to obtain a time-independent solution.

$$
\sigma_{t, g}(\vec{r}) \psi_{m, g}(\vec{r})=\sum_{g^{\prime}=1 m^{\prime}}^{2} w_{m^{\prime}} \sigma_{s, m^{\prime} \rightarrow m, g^{\prime} \rightarrow g}(\vec{r}) \psi_{m^{\prime}, g^{\prime}}(\vec{r})+\frac{\chi_{g}}{4 \pi k} \sum_{g^{\prime}=1}^{2} v \sigma_{f, g^{\prime}}(\vec{r}) \phi_{g^{\prime}}(\vec{r}), \quad g=\{1,2\}
$$

We have also set the streaming term to zero because we are in an infinite medium and there is no leakage from the system. Removing the differential streaming term from the transport equation leaves us with a system of two algebraically linear equations (an equation for each group). Equation (108) is specific to our 2-group infinite medium system.

This eigenvalue problem has purely isotropic scattering so we can replace the anisotropic scattering cross section with an isotropic one. Once we pull the scattering cross section term outside of the angle summation we can use the definition of scalar flux from equation (4) to eliminate angular fluxes from the RHS of equation (108).

$$
\sigma_{t, g}(\vec{r}) \psi_{m, g}(\vec{r})=\frac{1}{4 \pi} \sum_{g^{\prime}=1}^{2} \sigma_{s, g^{\prime} \rightarrow g}(\vec{r}) \phi_{g^{\prime}}(\vec{r})+\frac{\chi_{g}}{4 \pi k} \sum_{g^{\prime}=1}^{2} v \sigma_{f, g^{\prime}}(\vec{r}) \phi_{g^{\prime}}(\vec{r}), \quad g=\{1,2\}
$$

Next we can integrate these two equations over all angles. After some algebraic manipulation our system has the form:

$$
\mathbf{A} \vec{\phi}=k \vec{\phi}
$$

This is the standard form for an eigenvalue problem. The associated operator matrix and the eigenvector are defined below.

$$
\begin{aligned}
& \mathbf{A}=\left[\begin{array}{cc}
\sigma_{t, g 1}(\vec{r})-\sigma_{s, g 1 \rightarrow g 1}(\vec{r}) & -\sigma_{s, g 2 \rightarrow g 1}(\vec{r}) \\
-\sigma_{s, g 1 \rightarrow g 2}(\vec{r}) & \sigma_{t, g 2}(\vec{r})-\sigma_{s, g 2 \rightarrow g 2}(\vec{r})
\end{array}\right]^{-1}\left[\begin{array}{cc}
\chi_{g 1} v \sigma_{f, g 1}(\vec{r}) & \chi_{g 1} v \sigma_{f, g 2}(\vec{r}) \\
\chi_{g 2} v \sigma_{f, g 1}(\vec{r}) & \chi_{g 2} v \sigma_{f, g 2}(\vec{r})
\end{array}\right] \\
& \vec{\phi}=\left[\begin{array}{c}
\phi_{g 1}(\vec{r}) \\
\phi_{g 2}(\vec{r})
\end{array}\right]
\end{aligned}
$$

The eigenvalues of this system are $k$ such that $\operatorname{det}(\mathbf{A}-k \mathbf{I})=0$. Solving this system will produce two values for $k$. We are interested in the larger of these two, which is called the multiplication factor of the physical system. For the material data given in equation (4) the analytic infinite medium k-eigenvalue is $k_{\infty}=2.01185$.

When solved with an $\mathrm{S}_{6}$ fully level-symmetric quadrature set, our 3D UCBL scheme produces the solution $k_{\infty}=2.01182$ which is very slightly different than the analytic solution. The discrepancy in the solution is an artifact of the reflective boundary condition algorithm that we have devised for arbitrarily oriented surfaces. We had to develop this algorithm for arbitrary reflection such that the reflected direction is not a member of the discrete-ordinate set of directions. We believe that this algorithm is responsible for the error in our solution because the zone-averaged scalar flux (shown in Figure 14) is not flat, as it should be for an infinite medium problem. It is clear from this figure that the solution is too large in the boundary cells. The same feature is found in the single group solution for both energy groups. 
The reflective boundary algorithm used in this problem preserves the zero net current across the problem boundary, but it does not preserve the isotropic angular distribution at the boundary (see Appendix B for details). For spherical problems the algorithm favors reflection into quadrature directions that are inward grazing to the surface. This feature artificially captures reflected angular fluxes into a boundary layer. To verify that the error in our solution was an artifact of our reflective boundary algorithm we ran this same problem with a white boundary condition in place of a truly reflective boundary condition. Using a white boundary condition forces the reflected angular flux to be completely isotropic. For this modified problem the volume-averaged scalar flux solution for this problem was flat and the computed keigenvalue matched the analytic solution to the requested precision of the numerical computation.

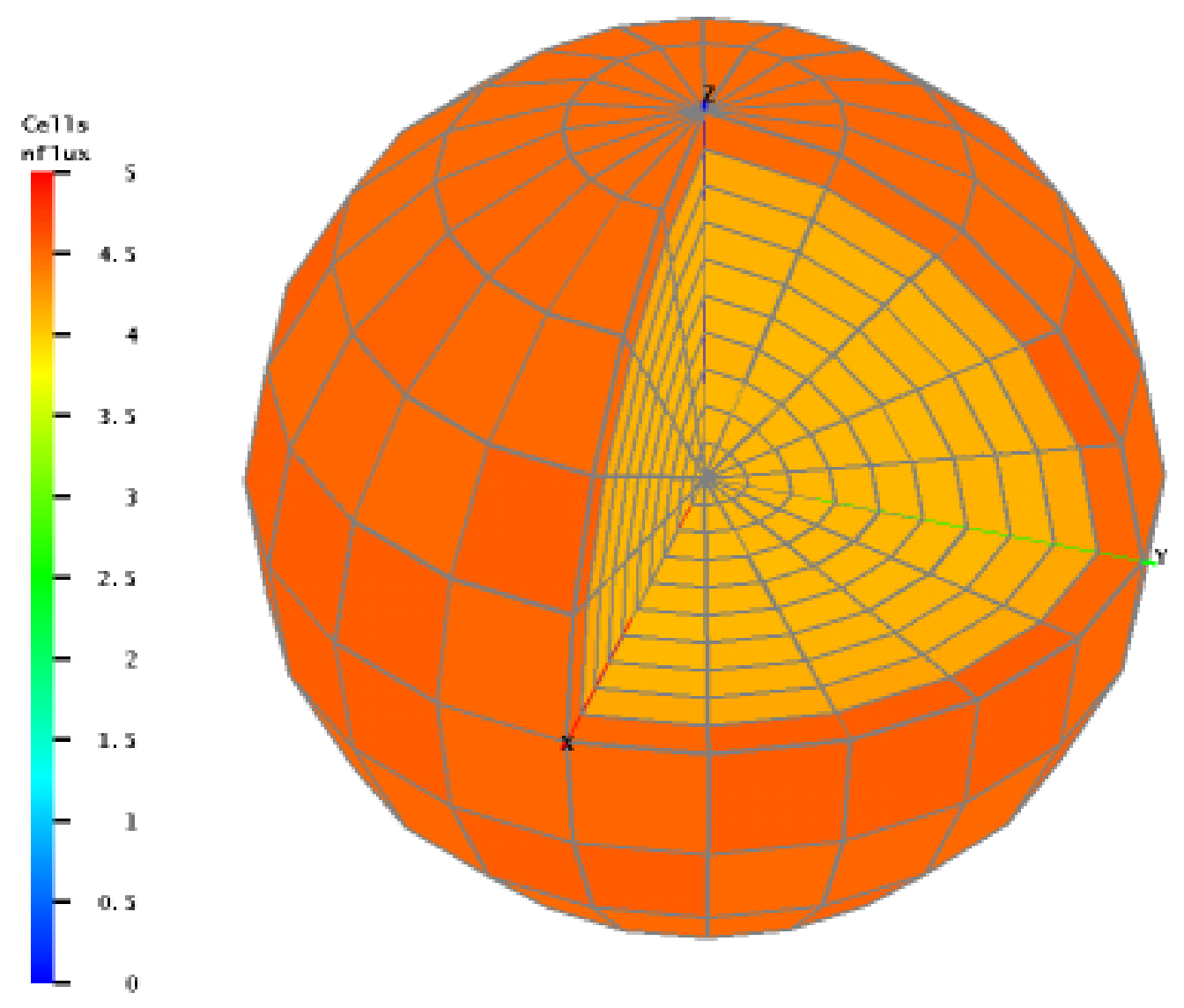

Figure 14. The scalar flux solution of the k-eigenvalue reflected sphere problem.

Forcing the boundary condition to preserve the isotropic solution cleared the discrepancy between the numerical and analytic solutions. This indicates that our discretization is accurately capturing the correct (flat) solution (see Figure 15). This result also demonstrated a weakness in our reflective boundary algorithm that needs to be addressed in the future. 
We can also compare the analytic ratio of group fluxes to the numerical results. We can manipulate equation (111) by solving for the ratio of the group fluxes for both balance equations in this system. Doing this and substituting in for the analytic value for $k$ and the material properties from equation (4) we find the following analytic ratio:

$$
\frac{\phi_{g 2}}{\phi_{g 1}}=\frac{k\left(\sigma_{t, g 1}-\sigma_{s, g 1 \rightarrow g 1}\right)-\chi_{g 1} v \sigma_{f, g 1}}{\chi_{g 1} v \sigma_{f, g 2}+k \sigma_{s, g 2 \rightarrow g 1}}=\frac{\chi_{g 2} v \sigma_{f, g 1}+k \sigma_{s, g 1 \rightarrow g 2}}{k\left(\sigma_{t, g 2}-\sigma_{s, g 2 \rightarrow g 2}\right)-\chi_{g 2} v \sigma_{f, g 2}}=1.00807
$$

The numerical results from case with the white boundary condition captures this same ratio.

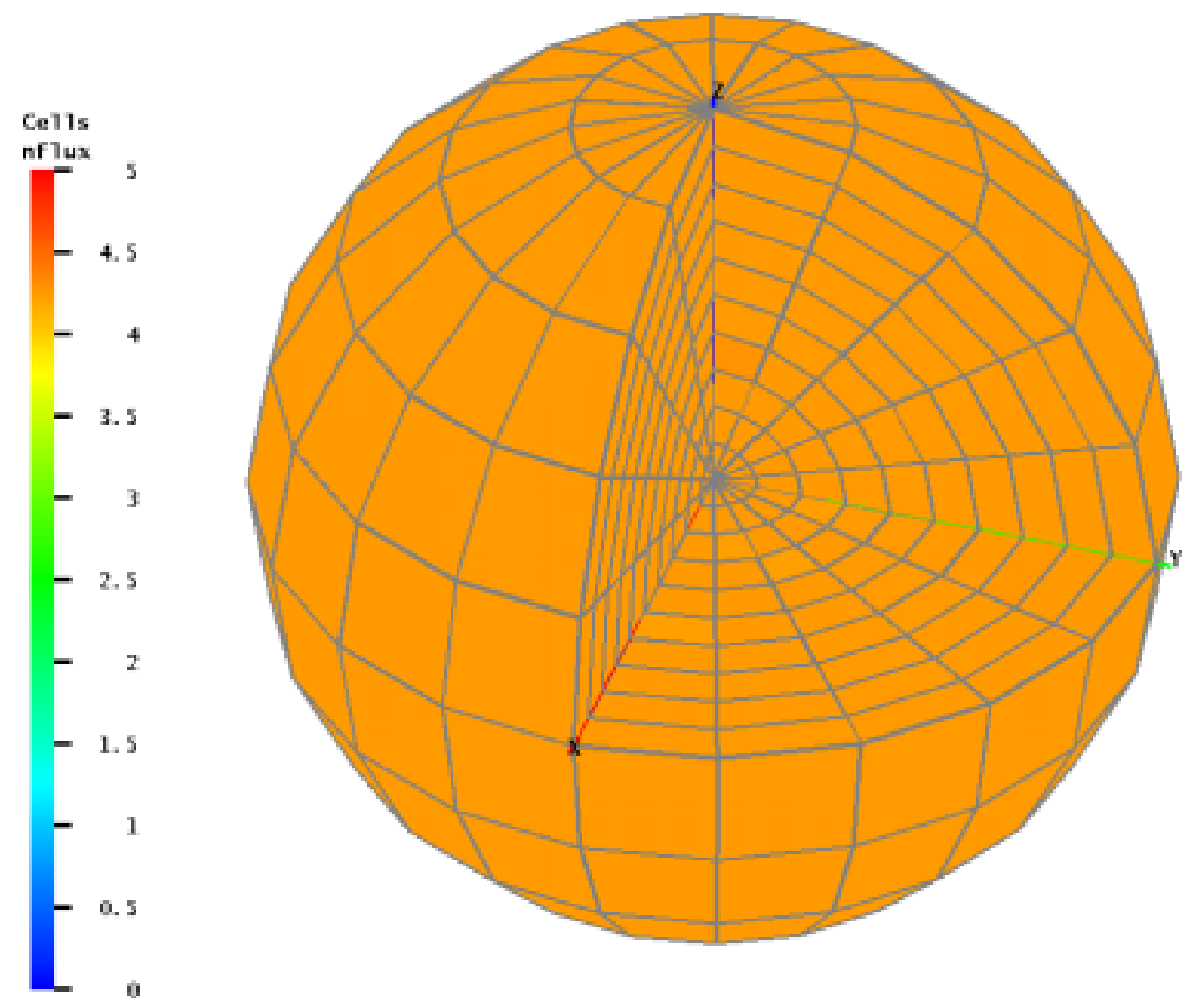

Figure 15. The scalar flux solution of the k-eigenvalue sphere problem with white boundary conditions.

This problem is not a stressful test of a spatial discretization scheme, for the correct solution is simply constant. However, it does demonstrate the ability of 3D UCBL to operate on an unstructured hexahedral/pyramid mixed mesh, and also our implementation of reflective boundary conditions on arbitrarily oriented faces. 


\section{B. Alpha Eigenvalue Problem}

We can also solve an alpha-eigenvalue, two group, infinite-medium problem with the same material properties as the problem evaluated in Section IV. B. (see equation (4)). In this problem we only consider a brick geometry so that our solution is not affected by the potential inaccuracy in the reflective boundary algorithm used in the reflected-sphere problem.

The infinite-medium analytic solution can be derived from the analytic transport equation where we replace the time-dependant angular flux with a separable solution.

$$
\psi(\vec{r}, \vec{\Omega}, E, t)=\psi_{\alpha}(\vec{r}, \vec{\Omega}, E) e^{\alpha t}
$$

Assuming that the distributed source is zero, the scattering term is isotropic and noting that the streaming term is zero for infinite medium problems our transport equation simplifies after integrating over the unit sphere.

$$
\left[\sigma_{t}(\vec{r}, E)+\frac{\alpha}{v(E)}\right] \phi(\vec{r}, E)=\int_{E^{\prime}=0}^{\infty} d E^{\prime} \sigma_{s}\left(\vec{r}, E^{\prime} \rightarrow E\right) \phi\left(\vec{r}, E^{\prime}\right)+\chi(E) \int_{E^{\prime}=0}^{\infty} d E^{\prime} v \sigma_{f}\left(\vec{r}, E^{\prime}\right) \phi\left(\vec{r}, E^{\prime}\right)
$$

We now apply the multi-group approximation for our two-group data. This approximation produces a system of 2 equations that can be formulated as an eigenvalue problem.

$$
\mathbf{A} \vec{\phi}=\alpha \vec{\phi}
$$

The associated operator matrix and the eigenvector are defined below:

$$
\begin{aligned}
& \mathbf{A}=\left[\begin{array}{cc}
v_{g 1} \sigma_{s, g 1 \rightarrow g 1}(\vec{r})+v_{g 1} \chi_{g 1} v \sigma_{f, g 1}(\vec{r})-v_{g 1} \sigma_{t, g 1}(\vec{r}) & v_{g 1} \sigma_{s, g 2 \rightarrow g 1}(\vec{r})+v_{g 1} \chi_{g 1} v \sigma_{f, g 2}(\vec{r}) \\
v_{g 2} \sigma_{s, g 1 \rightarrow g 2}(\vec{r})+v_{g 2} \chi_{g 2} v \sigma_{f, g 1}(\vec{r}) & v_{g 2} \sigma_{s, g 2 \rightarrow g 2}(\vec{r})+v_{g 2} \chi_{g 2} v \sigma_{f, g 2}(\vec{r})-v_{g 2} \sigma_{t, g 2}(\vec{r})
\end{array}\right] \\
& \vec{\phi}=\left[\begin{array}{c}
\phi_{g 1}(\vec{r}) \\
\phi_{g 2}(\vec{r})
\end{array}\right]
\end{aligned}
$$

The eigenvalues of this system are the solutions of $\operatorname{det}(\mathbf{A}-\alpha \mathbf{I})=0$. In addition to the material data given in equation (4), we must also define the group velocities.

$$
\vec{v}=\left[\begin{array}{l}
2.665912 \\
0.097452
\end{array}\right] \mathrm{cm} / \mathrm{ns}
$$

Once again this system will produce two eigenvalues. We are interested in the larger of these two, which describes the long-term behavior of the physical system. Using this material data the analytic infinite medium $\alpha$-eigenvalue is $\alpha_{\infty}=0.0973389 / \mathrm{ns}$.

When solved with the $S_{6}$ fully level-symmetric quadrature set, our 3D UCBL scheme produces the numerical solution $\alpha_{\infty}=0.0973388 / \mathrm{ns}$ which matches exactly to the requested precision of the calculation $\left(\varepsilon=10^{-5}\right)$. For this solution the group scalar fluxes are flat, as they should be, and the ratio of the group 1 flux to the group 2 flux matches the analytic ratio to 6 significant figures.

$$
\frac{\phi_{g 1}}{\phi_{g 2}}=\frac{\sigma_{s, g 2 \rightarrow g 1}+\chi_{g 1} v \sigma_{f, g 2}}{\sigma_{t, g 1}+\frac{\alpha}{v_{g 1}}-\sigma_{s, g 1 \rightarrow g 1}-\chi_{g 1} v \sigma_{f, g 1}}=\frac{\sigma_{t, g 2}+\frac{\alpha}{v_{g 2}}-\sigma_{s, g 2 \rightarrow g 2}-\chi_{g 2} v \sigma_{f, g 2}}{\sigma_{s, g 1 \rightarrow g 2}+\chi_{g 2} v \sigma_{f, g 1}}=2.3913
$$


Again, this is not a stressful test of our discretization but it does demonstrate our ability to calculate static $\alpha$-eigenvalues accurately.

\section{C. Critical Thickness of an Anisotropic Slab-like Problem}

To demonstrate our performance for anisotropic problems we compare numerical results to semianalytic solutions for a critical slab $(k=1)$ problem. For this problem we consider a one-group model for $\mathrm{Pu}-239$ that has the following material data:

$$
\begin{aligned}
\sigma_{t} & =1.0 & & \sigma_{s 0}=0.733333 \\
\sigma_{a} & =0.0 & & \sigma_{s 1}=0.2 \\
v \sigma_{f} & =0.66667 & & \sigma_{s 2}=0.075
\end{aligned}
$$

The infinite medium k-eigenvalue for this problem does not depend on the anisotropic scattering cross sections and has a value of $k_{\infty}=2.5$. The critical slab thickness for both the $\mathrm{P}_{1}$ and $\mathrm{P}_{2}$ problems have been evaluated. ${ }^{24}$ For the $\mathrm{P}_{1}$ problem the critical half-thickness is $0.77032 \mathrm{~cm}$ and $0.76378 \mathrm{~cm}$ for the $\mathrm{P}_{2}$ case, where the results are supposed to be accurate to the digits given.

We generate numerical results for this problem by running a full 3D brick geometry with reflective boundary conditions on 5 of the brick's faces. This simulates a 1D half-thickness calculation. Since our code does not currently have the ability to compute critical dimension searches we simply set up the problem to have the critical thickness and then compare the computed eigenvalue to the analytic solution $k=1.0$.

For the infinite-medium case the k-eigenvalue is exactly 2.5 as expected. For the $\mathrm{P}_{2}$ problem the 3D UCBL-computed value for $k$ is in error by $0.304 \%$ for an $\mathrm{S}_{16}$ calculation with 50 cells in the $\mathrm{x}$-dimension. A similarly discretized problem was run in the transport code PARTISN using the ED spatial discretization; this produced a numerical result that was in error by $0.406 \%$. We note that these codes use different quadrature sets and that the solution appears to be resolved in space because increasing the number of cells did not significantly alter the computed eigenvalue. The error in these solutions may be attributed to the solution being unresolved in angle. Since each code uses its own quadrature set we expect some slight variation in the errors even when the same number of quadrature directions are used.

Again this problem is not an exhaustive test of our scheme's characteristics. However, it does demonstrate this scheme's numerical accuracy for finite-volume eigenvalue calculations and anisotropic scattering model.

\section{D. Coarsely Discretized Multi-Region Problem}

This test is designed to expose weaknesses in spatial-discretization methods. As shown in Figure 16 this problem has 5 regions. The region on the left has a distributed isotropic source and a scattering ratio of 
unity. There are two strong absorbers. One is next to the top part of the source region and the other is in the lower right of the problem. Along the bottom of the problem between this second strong absorber and the source region is a very weakly absorbing (near vacuum) region. The remainder of the problem is a highly scattering material.

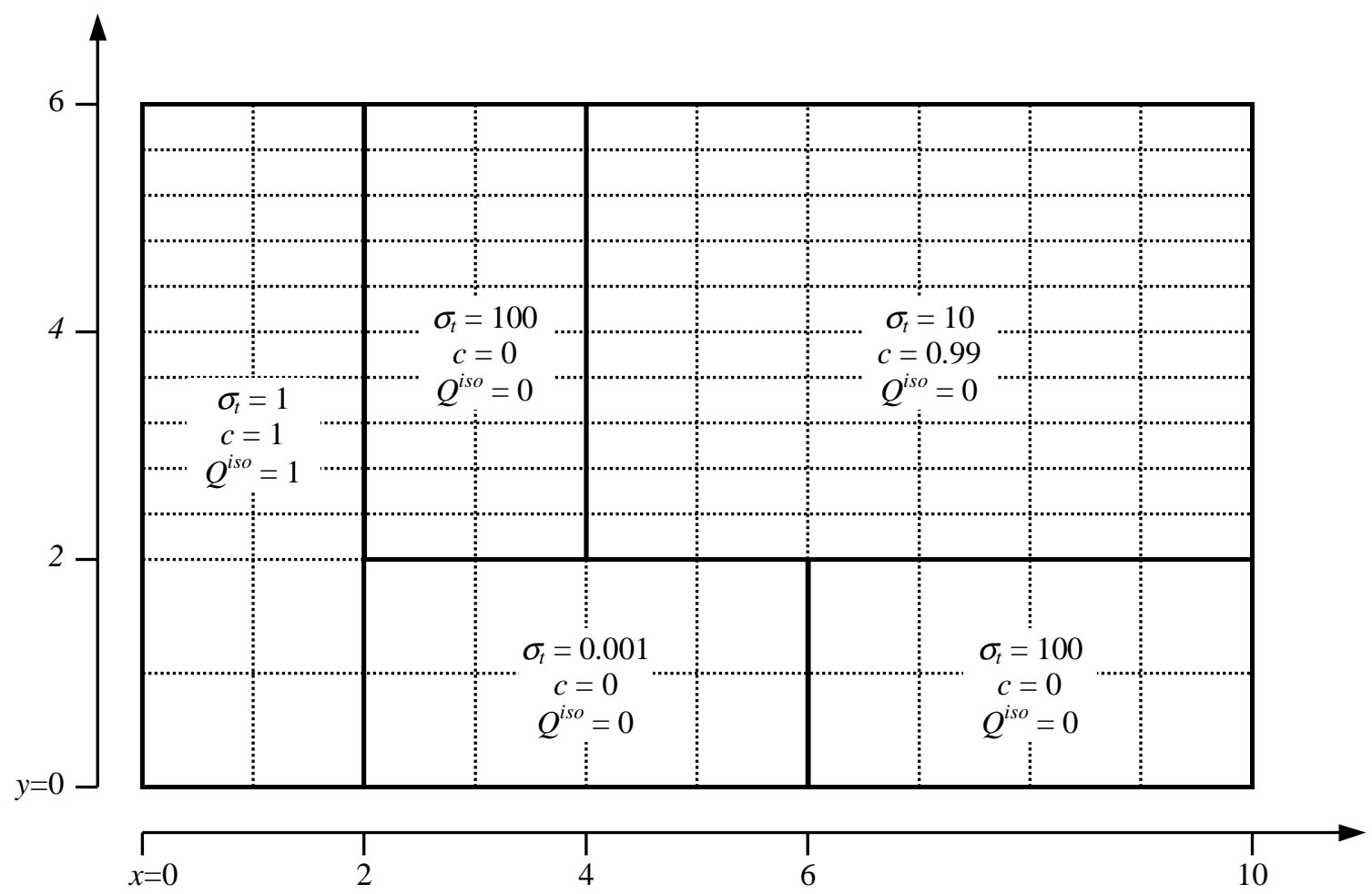

Figure 16. Geometry and material map for the coarsely discretized multi-region problem.

Physically, most particles are born in the source region and then are lost to leakage (all boundaries are vacuum) or absorption in the strongly absorbing regions. The more interesting particles are born in the lower part of the source region, stream through the thin region, and impinge on the diffusive region. This streaming introduces a boundary layer in the diffusive region that is not resolved by the very coarse spatial grid we impose on the problem. Most transport discretizations have trouble handling this unresolved boundary layer, producing inaccurate or even negative solutions.

We have solved this 3D problem using 3D Diamond Difference, DD, with flux fixups, 3D Exponential Discontinuous, ED, and 3D UCBL. The same $\mathrm{S}_{4}$ quadrature set was used for all cases. The spatial grid is indicated by dotted lines in Figure 16. The problem is uniform in the $z$-direction and is discretized into 10 cell layers of $\Delta z=1 \mathrm{~cm}$ each. All boundary conditions are vacuum. We will examine the solution in the $\mathrm{XY}$ plane near the middle of the problem in the $z$-direction. 
We display logarithmic-contour depictions of the 3D UCBL, ED and DD solutions in Figures 17, 18, 19 and 20. The major contours in these plots are logarithmically spaced and their values range from $10^{\circ}$ to $10^{-20}$; the numbers on the plot indicate the exponent in powers of ten $(0$ to -20$)$. There are 10 logarithmically spaced minor contour lines between each major contour line. The solution is highest in the source region and decreases to the right.

In the 3D UCBL case (see Figure 17,) we notice that the solution is reasonably monotonic, decreasing in value as we move to the right. We see a nice symmetry in the source region with particles streaming through the near vacuum region. The solution rapidly drops off in the two strongly absorbing regions without producing negative values. This is one of the strengths of our discretization. The solution in the thick diffusive region has the right shape but the solution is not as smooth as it should be. The wiggles in the contour lines are a result of plotting the corner-averaged values instead of the cell-averaged values. Plotting the solution using corner midpoint values would appear to be the correct thing to do since UCBL preserves balance on corner volumes. However, in our diffusion limit analysis of Chapter III, we showed that all corners about a common vertex would have the same value when the problem becomes thick and diffusive. We also know that the unknowns for diffusion solution that we limit to exit at vertex points and not at cell centers. This would indicate that at least for diffusive regions the corner flux values should be plotted at vertex points and not at cell centers.

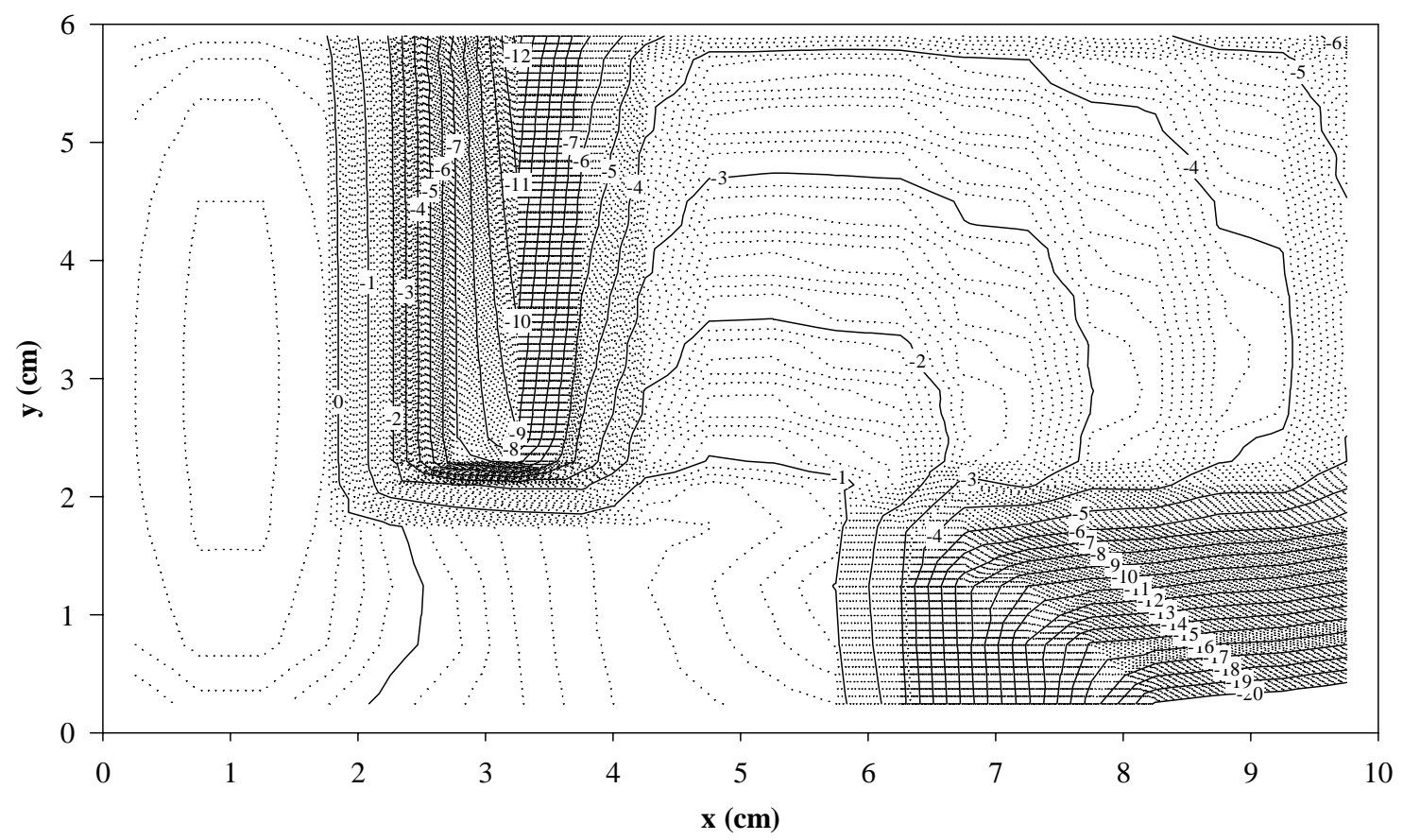

Figure 17. 3D UCBL corner midpoint solution for the coarsely discretized multi-region problem. 
In Figure 18 we plot the same UCBL solution at cell midpoints instead of at corner midpoints. We see all of the basic features in the cell midpoint solution that we discussed above for the corner midpoint solution. The only real difference is that the solution appears much smoother in the diffusive region. We still are not plotting the solution at vertex points but the cell average scalar flux is a volume-weighted average of the corner values. In diffusive regions, this cell centered flux looks like an average of vertex values and should be smooth just as we show in Figure 18. It appears that the solution does not drop as much in the first strongly absorbing region when compared to the solution shown in Figure 17. However, both of these plots present the same data. It is just presented in different formats.

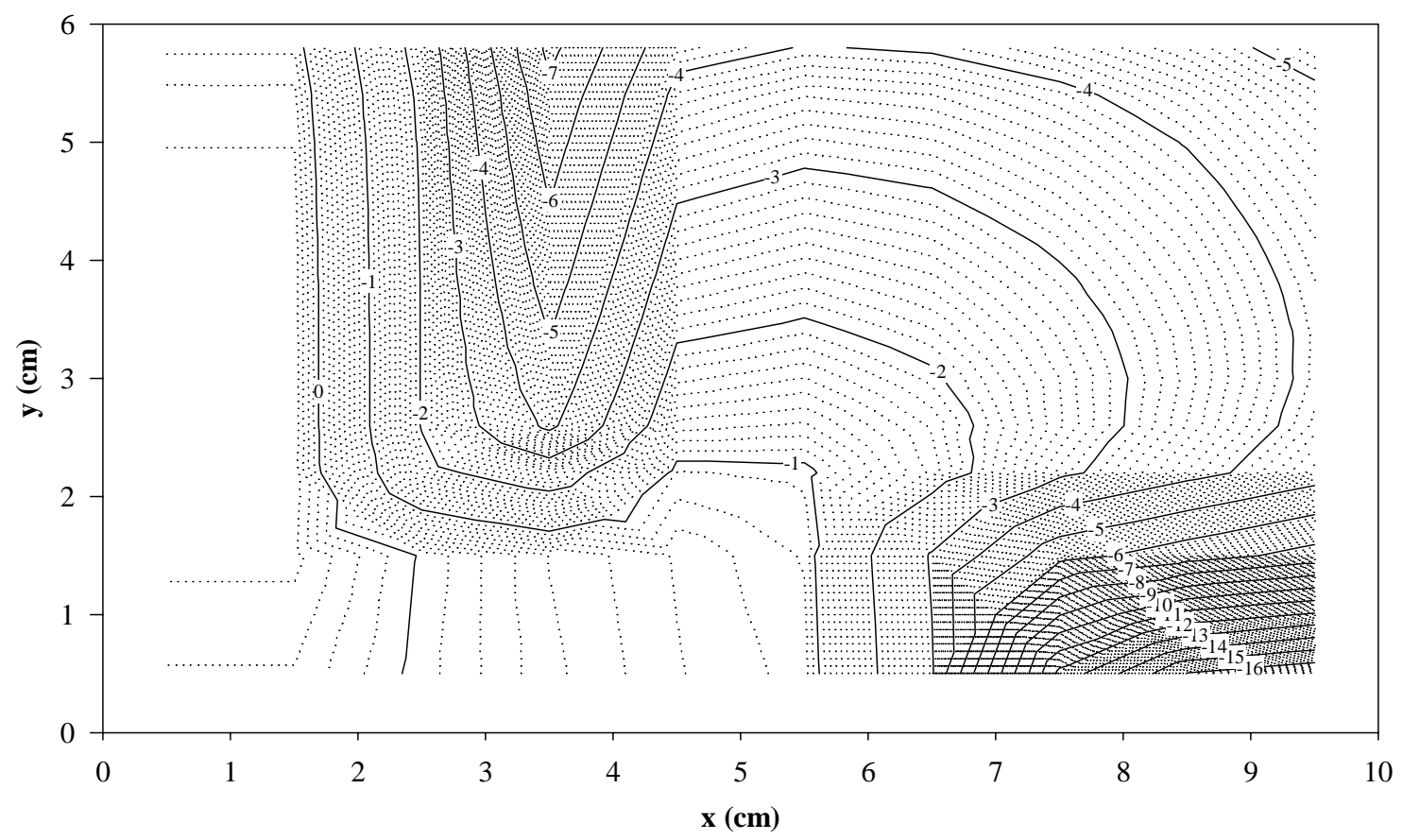

Figure 18. 3D UCBL cell midpoint solution for the coarsely discretized multi-region problem.

The "ED" solution is shown in Figure 19. We used the PARTISN code to find both the "ED" and the DD solutions. The "ED" solution has many of the same general features as the UCBL solution. The solution is largest in the source region and diminishes to the right. Most of the particles flow through the near vacuum region into the thick diffusive region. Interestingly enough the solution is negative in the two strongly absorbing regions - even though ED is a purely positive scheme. We believe that this is a result of the Newton-Raphson solver not converging completely in the PARTISN implementation. ${ }^{25}$ We have referred to this limited implementation of ED by using quotes.

In the thick diffusive region the "ED" solution is slightly smaller than the UCBL solution and is smoother than the UCBL corner midpoint solution; however, this plot was generated by plotting cellaverage fluxes at cell midpoints. We also notice that in the strongly absorbing regions the solution appears 
to be shifted the right when compared to the model shown in Figure 16. This feature is also seen in the UCBL solution although the sharp gradients in the "ED" solution make it easier to see. This artifact is a result of plotting cell midpoints on a very coarsely discretized grid. The data is only known at two distinct $x$-coordinates and the plotting software presents evenly spaced contours between these data points. The result is that the solution appears shifted by half a cell width to the right. Additionally the solution near the inside corners of the absorbing regions appears to be slightly larger than the UCBL solution

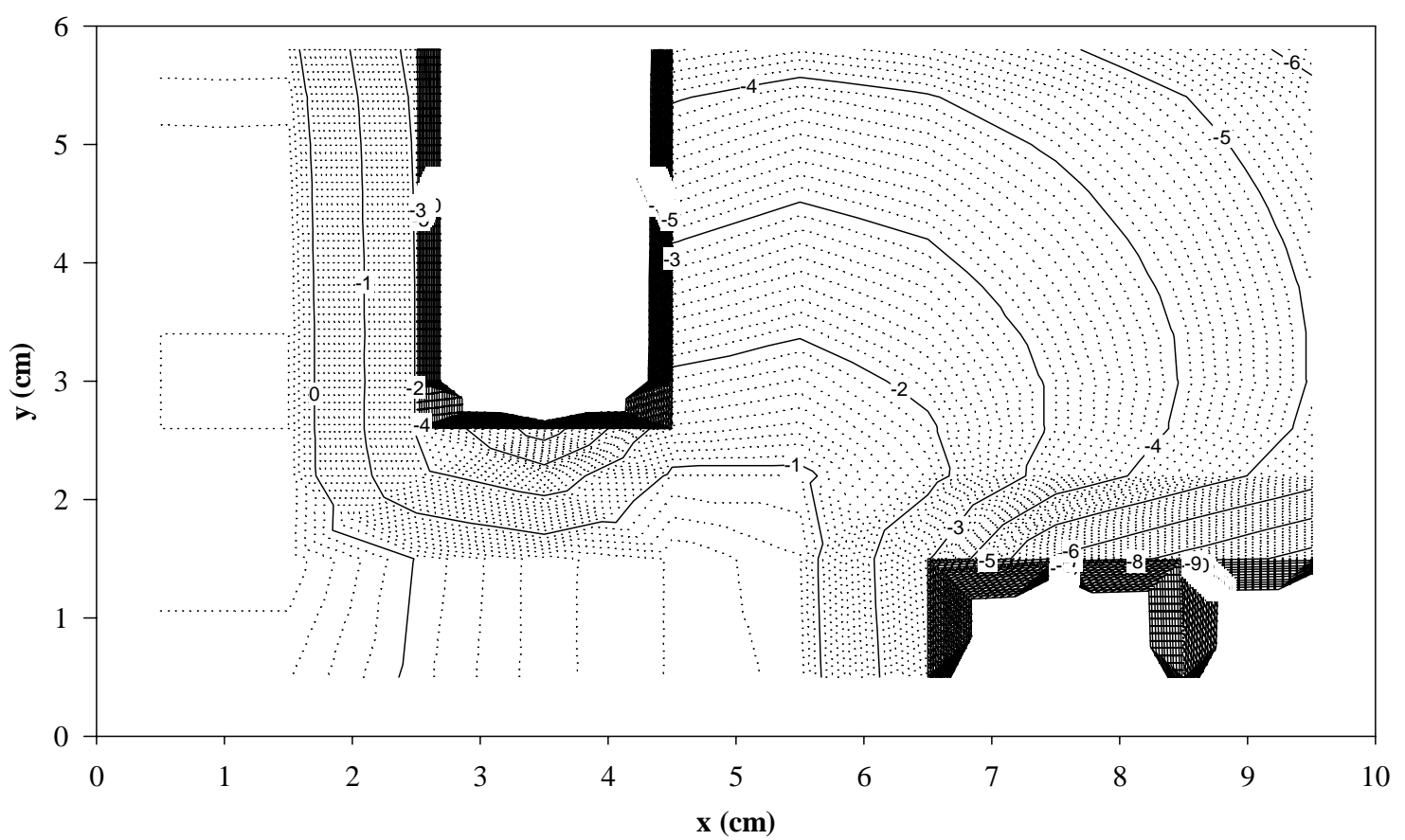

Figure 19. The ED solution for the coarsely discretized multi-region problem.

The DD with flux fixups solution is shown in Figure 20. The solution is strictly non-negative because of the fixups but it is exactly zero in portions of both of the strongly absorbing regions (which is not too far away from the true solution in an absolute sense). We have doubled the spatial resolution in each dimension so that we have an equal number of unknowns per unit volume when compared to the UCBL problem. The solution in the source region is symmetric and most of the particles are flowing through the streaming region into the diffusive region. The solution in the diffusive region lies between the UCBL and ED solutions. The solution in this region is smoother than the UCBL solution. (Again, we are plotting cell-averaged solutions.) However, DD displays unphysical behavior in the sharp boundary layers near the strongly absorbing regions. We also see what appears to be an unphysical reflection of particles off of the second absorbing region. 
In summary, this numerical test demonstrates many characteristics of 3D UCBL. It does a remarkable job of capturing a strictly positive solution even in coarse grids where strong boundary layers exist. It also appears to do a very nice job of capturing these boundary layers where they exist when compared to the shifted solution that ED produces. We do not know how accurate our solution is in this problem. However, the solution is comparable in magnitude to the ED and DD solutions.

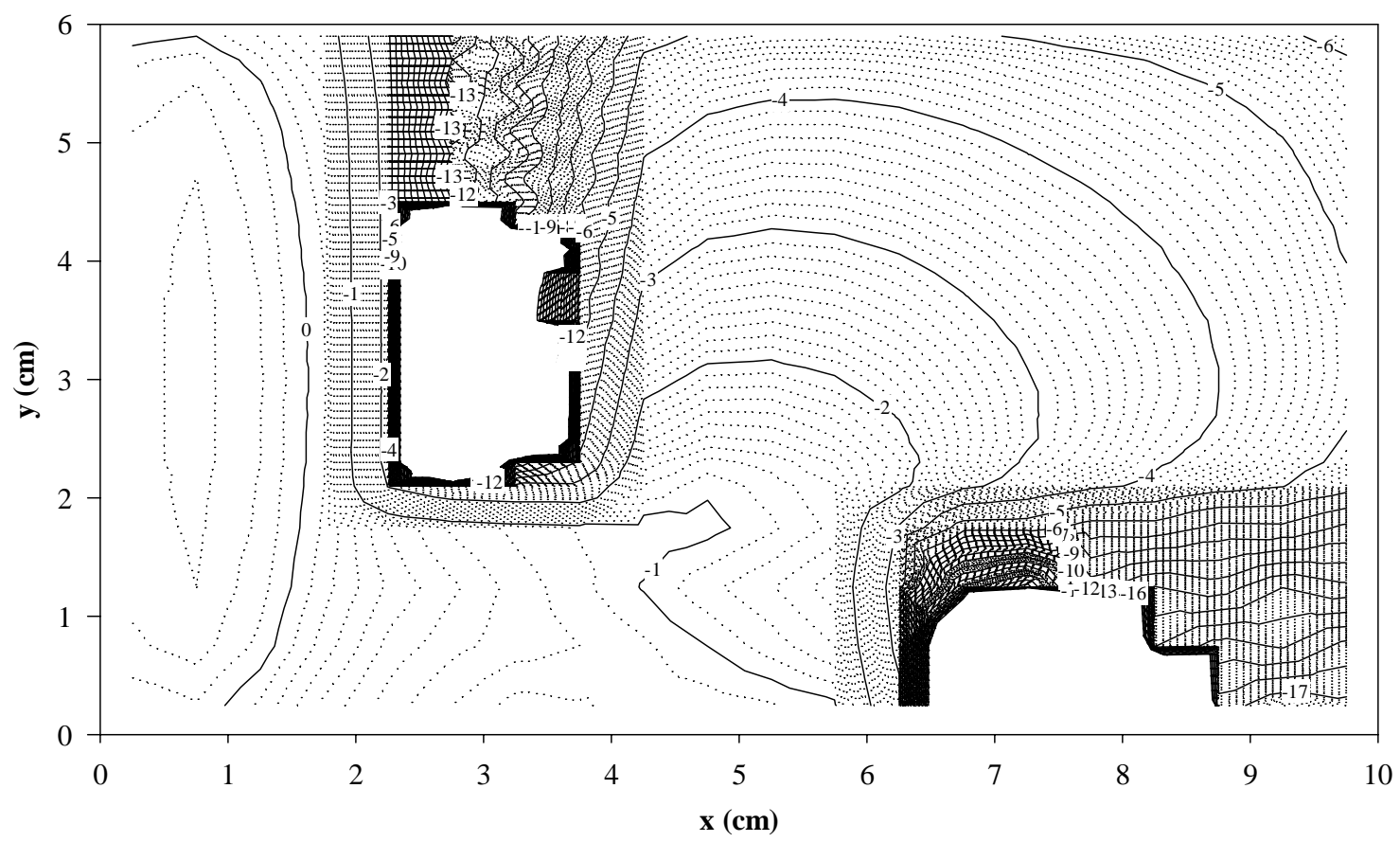

Figure 20. The DD solution with fixups solution for the coarsely discretized multi-region problem.

\section{E. Accuracy in Thin to Intermediately Thick Problems.}

This test problem is very similar to the 1D truncation error analysis problem run in Chapter III as it examines the truncation error and overall quality of the UCBL solution compared to LD and DD. However, this is a truly $3 \mathrm{D}$ problem. We consider a source-free brick, 7 × 5 × 3 mean-free paths, with an isotropic incident intensity on the minimum $x$-face. The removal cross section is half of the total. In each problem we used the same $\mathrm{S}_{4}$ level-symmetric quadrature set. In all cases we were very careful to use exactly the same quadrature set so that there would be no differences caused by the angular discretization. All boundaries are vacuum. The solution was considered converged when the point wise maximum error norm between Richardson iterations was less than $10^{-6}$. We compute the exiting flow rate from one-quarter of the $\max x$-face for various mesh spacings. In Table III we present errors in the solution for UCBL, DD (with no negative flux fixups) and LD where a $128 \times 128 \times 128 \mathrm{LD}$ case is used as the reference solution. We 
present $\mathrm{DD}$ and LD results because both schemes are widely used and LD is known to be a reasonably accurate method, and thus serves as a known standard against which to compare the others.

As shown in Table III the LD solution is too low for coarse meshes and approaches the correct value as we refine the mesh. The DD solution likewise approaches the reference solution from below with the exception of the solution for the coarsest mesh. The first DD data point is very close to the reference solution and so it appears at first glance that the DD solution gets worse as the mesh is refined. In fact this is not the case. DD is simply very lucky in this case probably due to cancellation of error. As we can see in the Figure 21 below the DD solution follows the expected trend for the remaining data points. In this plot we show the error in the leakage rate when compared to the resolved LD solution. The $x$-axis in this plot shows the power of 2 for the number of cells used in each dimension. Differing from the first two discretizations the 3D UCBL solution approaches the reference solution from above. We also notice that the absolute error for the UCBL solution is larger than the LD or DD errors for coarse meshes but is equal to or better than the DD solution for fine meshes.

Table III

Errors in the Exiting Flow Rate for Problem IV. F.

\begin{tabular}{|c|ccc|ccc|}
\hline \multirow{2}{*}{$\begin{array}{c}\text { Number of } \\
\text { cells }\end{array}$} & \multicolumn{3}{|c|}{ Error $\times 10^{-3}$} & \multicolumn{3}{c|}{ Error Reduction Ratio } \\
\cline { 2 - 7 } & LD & DD & UCBL & LD & DD & UCBL \\
\hline $2 \times 2 \times 2$ & & & 3.232 & & & \\
$4 \times 4 \times 4$ & -0.340 & 0.017 & 0.767 & & & 4.22 \\
$8 \times 8 \times 8$ & -0.119 & -0.212 & 0.256 & 2.85 & 0.08 & 3.00 \\
$16 \times 16 \times 16$ & -0.013 & -0.138 & 0.106 & 9.40 & 1.54 & 2.41 \\
$32 \times 32 \times 32$ & -0.003 & -0.117 & 0.050 & 4.72 & 1.17 & 2.14 \\
$64 \times 64 \times 64$ & -0.002 & -0.085 & 0.025 & 1.43 & 1.38 & 2.02 \\
\hline
\end{tabular}

In our table we do not present LD or DD data for the $2 \times 2 \times 2$ case because the PARTISN code used to generate these two solutions does not allow problems of so few cells to be run. We also note that to improve the readability in the table we have multiplied the entries by $10^{3}$. We did not run any larger problems because of the excessive computing resources required to run such large problems.

In the second half of Table III we present the error reduction ratio. The error reduction ratio is the ratio of the current error to the error for the next coarser mesh. We present this information to demonstrate the order of truncation error for each method. This value is a good representation of the order of accuracy for the numerical implementation of each spatial discretization scheme. From this table we quickly notice that neither LD nor DD appear to be as accurate as a 1D analysis would predict. From this table it would appear that $\mathrm{LD}$ (not tri- $\mathrm{LD}$ ) is second-order accurate since, on average, the error in the solution is reduced by more than a factor of 4 but less than a factor of 8 each time the mesh resolution is doubled in each dimension. Similarly, it would appear that the DD scheme in PARTISN is less than first order accurate for this problem. 
When analyzing the DD rate of convergence we can throw out the first error-reduction ratio data point because of that lucky cancellation of error that DD somehow achieves for the coarsest mesh. But even without this data point the average reduction in error per doubling of the resolution is only about 1.4. The UCBL solution is clearly first-order accurate for general 3D problems as we claimed in Chapter III. We see that the error in the leakage current is reduced by about a factor of 2 each time the mesh doubles.

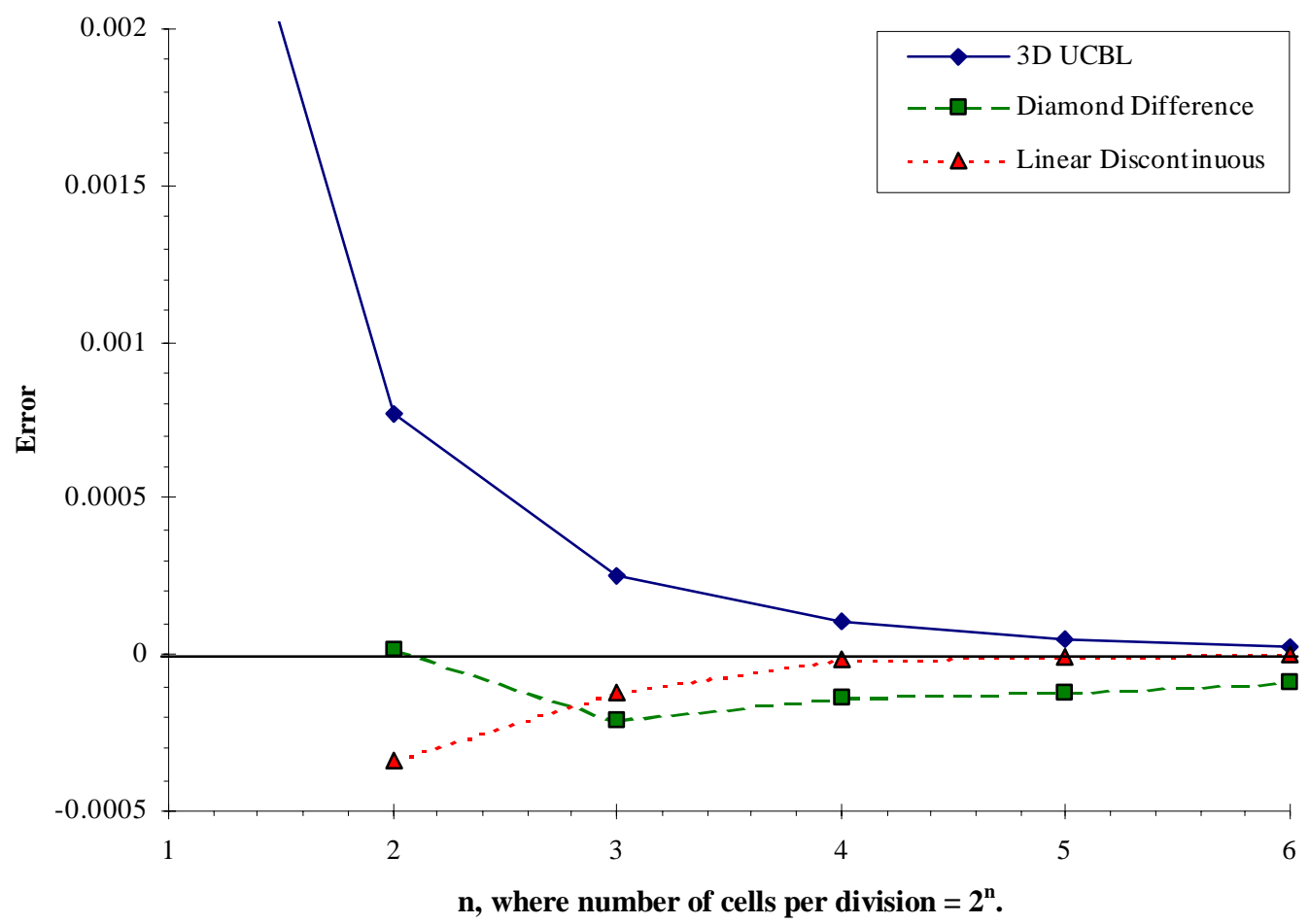

Figure 21. Linear-log plot of the leakage error as a function of 1D mesh refinement.

As an alternate view of this data we present the leakage errors on a log-log plot in Figure 22. In this plot the $x$-axis represents the total number of cells in the entire problem. This representation does a better job of presenting the error reduction as a function of 3D-mesh refinement. The slop of each of the lines is also a good indication of relative order of accuracy. The DD solution is not monotonic because of the lucky cancellation of error found on the coarsest mesh. If we discard this data point we notice that all of the error trends follow the same conclusions that we made above. The LD error reduces more quickly than either the DD or UCBL solution although its progress is not as smooth as we might expect. The UCBL solution appears first-order accurate and is very smooth. The DD trend is reasonably smooth if we discard the first data point. However, from this plot it would appear that DD has a lower order of accuracy than either UCBL or LD. 
We wanted to run this specific problem because it is a truly 3D extension of the problem examined in Chapter III. This simulation shows us that the UCBL scheme may produce larger errors than LD or DD in coarsely discretized problems. The results shown here complement those shown in Table II demonstrating that the UCBL scheme is a first-order accurate scheme for general 3D problems. However, it should be noted that for specific slab-like problems 3D UCBL behaves like a second-order scheme as we showed in Chapter III.

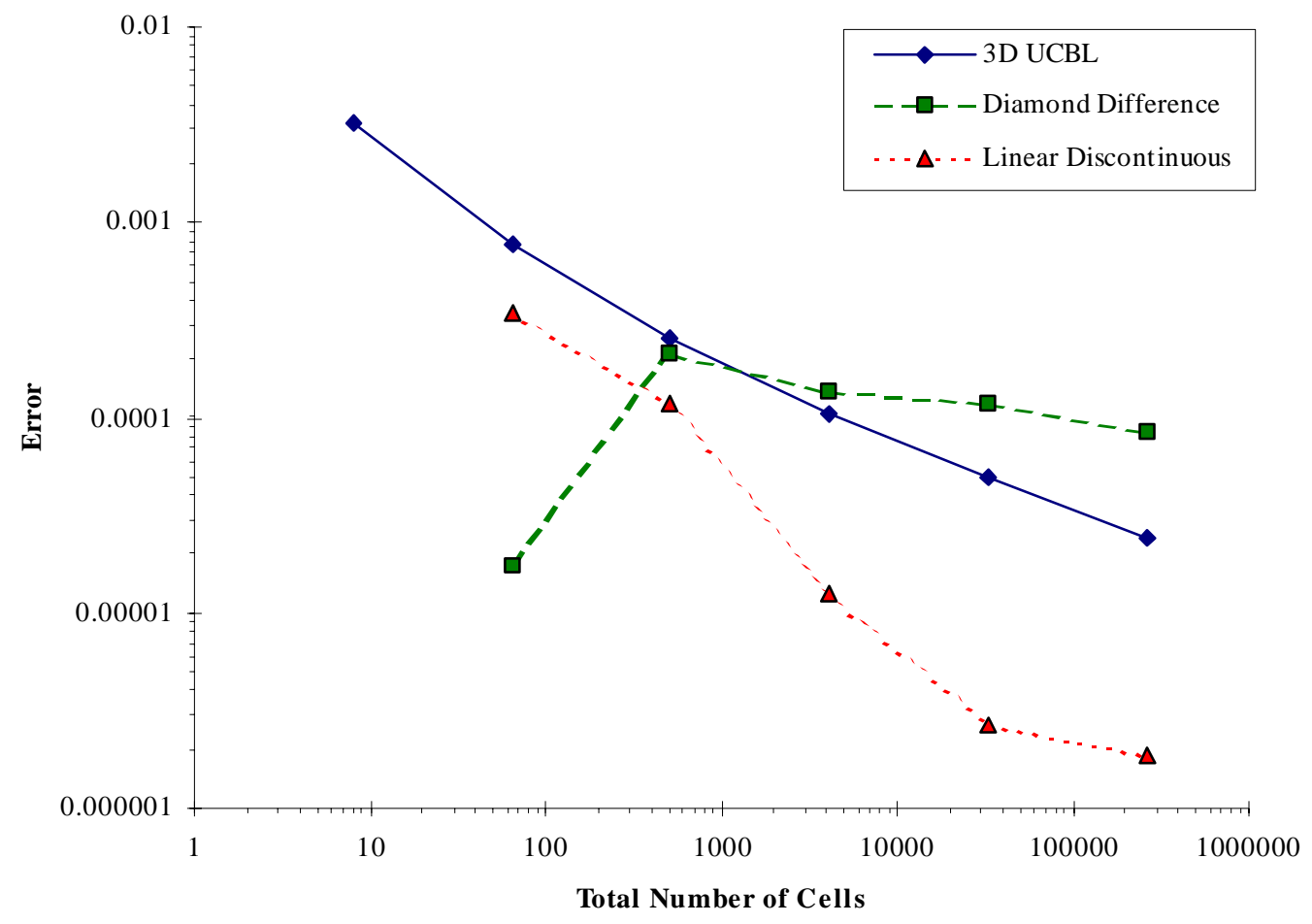

Figure 22. Log-log plot of the leakage error as a function of 3D mesh refinement.

\section{F. Comparison of SMaRT-Z Corner Balance to 3D UCBL}

The primary purpose of this problem is to test UCBL with an unstructured mesh and to compare its solution to that obtained by the SMaRT-Z corner balance (SZCB) discretization developed by Richardson. ${ }^{15}$ This discretization considers each z-level to be a 2 D problem but uses a 3D UCB discretization ${ }^{11}$ to solve the transport problem on each z-level. Communication between z-levels is done using upstream closures that are almost identical to the $2 \mathrm{D}$ closures. Between cells in the z-direction a step closure is used and between corners in a single cell an upstream closure is used. This scheme is restricted to grids whose cells are 
polygonal prisms with top and bottom faces parallel to the XY plane. The SMaRT-Z grid used in this problem meets this restriction.

The geometry of problem consists of three cylinders. Each smaller cylinder is contained inside of the larger one but not concentric with it. This assembly of three circles is placed into a rectangular region so that the boundary of our problem forms a brick. We vary the material properties in the z-direction of this $5.5 \mathrm{~cm}$ tall problem as shown in Table IV. We have also placed an isotropic source in the smallest cylinder between $\mathrm{z}=3.5$ and $4 \mathrm{~cm}$. The geometry of this problem is very similar to that of an oil well-logging tool but we are not trying to actually model such an instrument. We simply want to demonstrate that our discretization can utilize complex meshes and that it captures the same features as a more established discretization.

The grid we use has 204 2D SMaRT elements and 35 z-layers. The SMaRT elements are either triangles or quadrilaterals. For both spatial discretizations we use the same $\mathrm{S}_{8}$ level-symmetric quadrature set. The one-group isotropic cross sections shown in Table IV do not represent any real material but were chosen to demonstrate the performance of each scheme.

Table IV

Material Data for Circles in Circles Problem

\begin{tabular}{|l|c|c|}
\hline \multicolumn{1}{|c|}{ Material } & $\sigma_{\mathrm{t}}\left(\mathrm{cm}^{-1}\right)$ & $\sigma_{\mathrm{s}}\left(\mathrm{cm}^{-1}\right)$ \\
\hline Outer region & 2.0 & 1.0 \\
Large cylinder & 1.0 & 0.90 \\
Middle cylinder & 0.1 & 0.09 \\
Small cylinder: & & \\
z=0-1.5 cm & 0.1 & 0.09 \\
$\mathrm{z}=1.5-2 \mathrm{~cm}$ & 20.0 & 0.00 \\
$\mathrm{z}=2-3.5 \mathrm{~cm}$ & 0.1 & 0.09 \\
$\mathrm{z}=3.5-4 \mathrm{~cm}$ & 1.0 & 0.80 \\
$\mathrm{z}=4-5.5 \mathrm{~cm}$ & 0.1 & 0.09 \\
\hline
\end{tabular}

In Figures 23 and 24 we show the 3D UCBL and SZCB solutions, respectively, for four different perspectives. In all cases the solution varies by several orders of magnitude so we have chosen to present the logarithm of the solution. The range of solutions for both discretizations is $10^{-6}$ to $10^{-1} \mathrm{~cm}^{-2} \mathrm{sec}^{-1}$. The perspective in the upper right of each figure has the front of the grid removed so that we can see what the solution looks like in the interior of the problem. The lower-left and lower-right perspectives are XY planes at $\mathrm{z}=3.75 \mathrm{~cm}$ and $\mathrm{z}=1.75 \mathrm{~cm}$, respectively. The $\mathrm{XY}$ plot at $\mathrm{z}=3.75 \mathrm{~cm}$ cuts through the region where we have placed an isotropic volumetric source in the smallest cylindrical region. The plot at $\mathrm{z}=1.75 \mathrm{~cm}$ shows the solution though the plane were we have placed a strongly absorbing material in the smallest cylinder. 

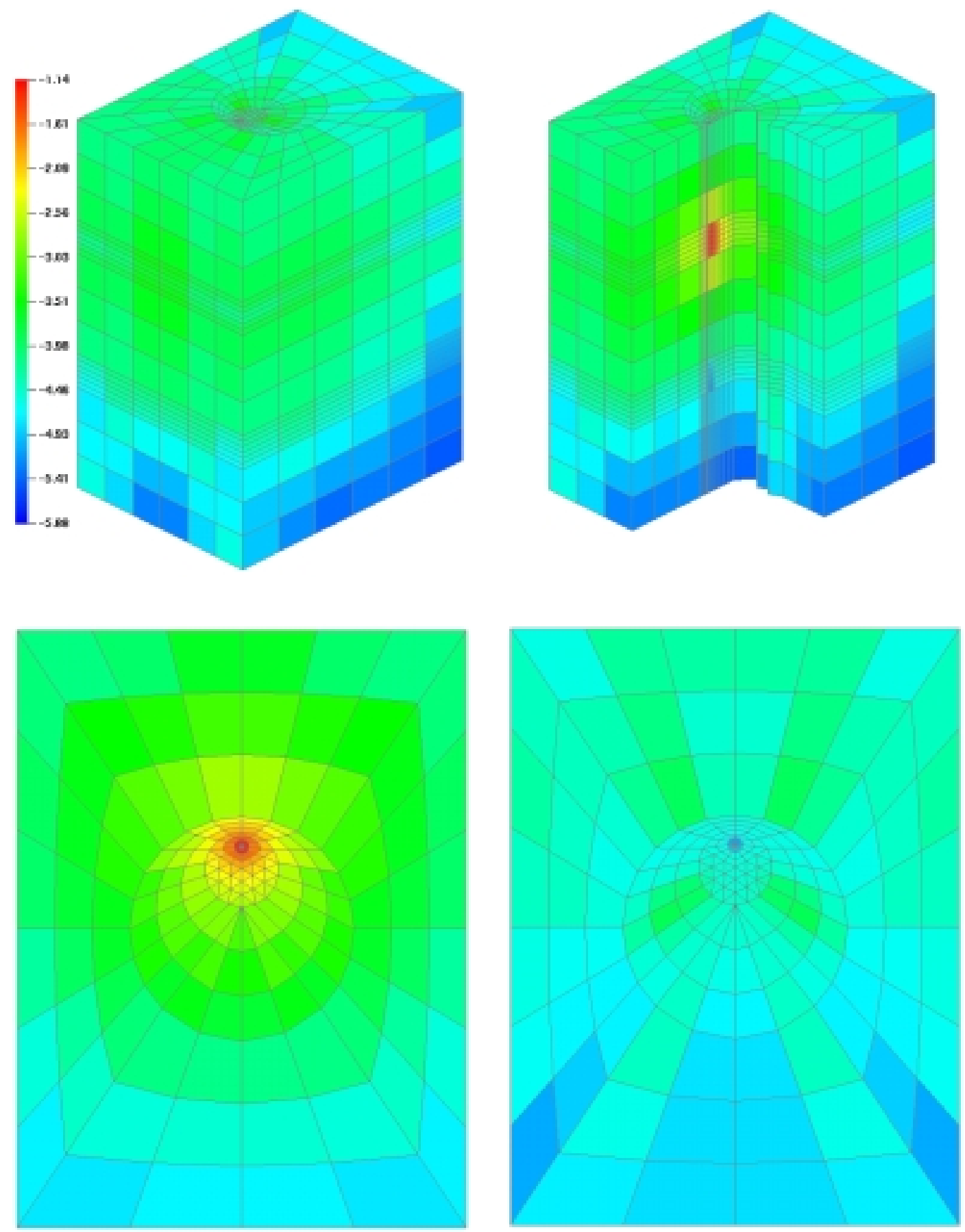

Figure 23. UCBL logarithmic solution for the circles-in-circles problem. 

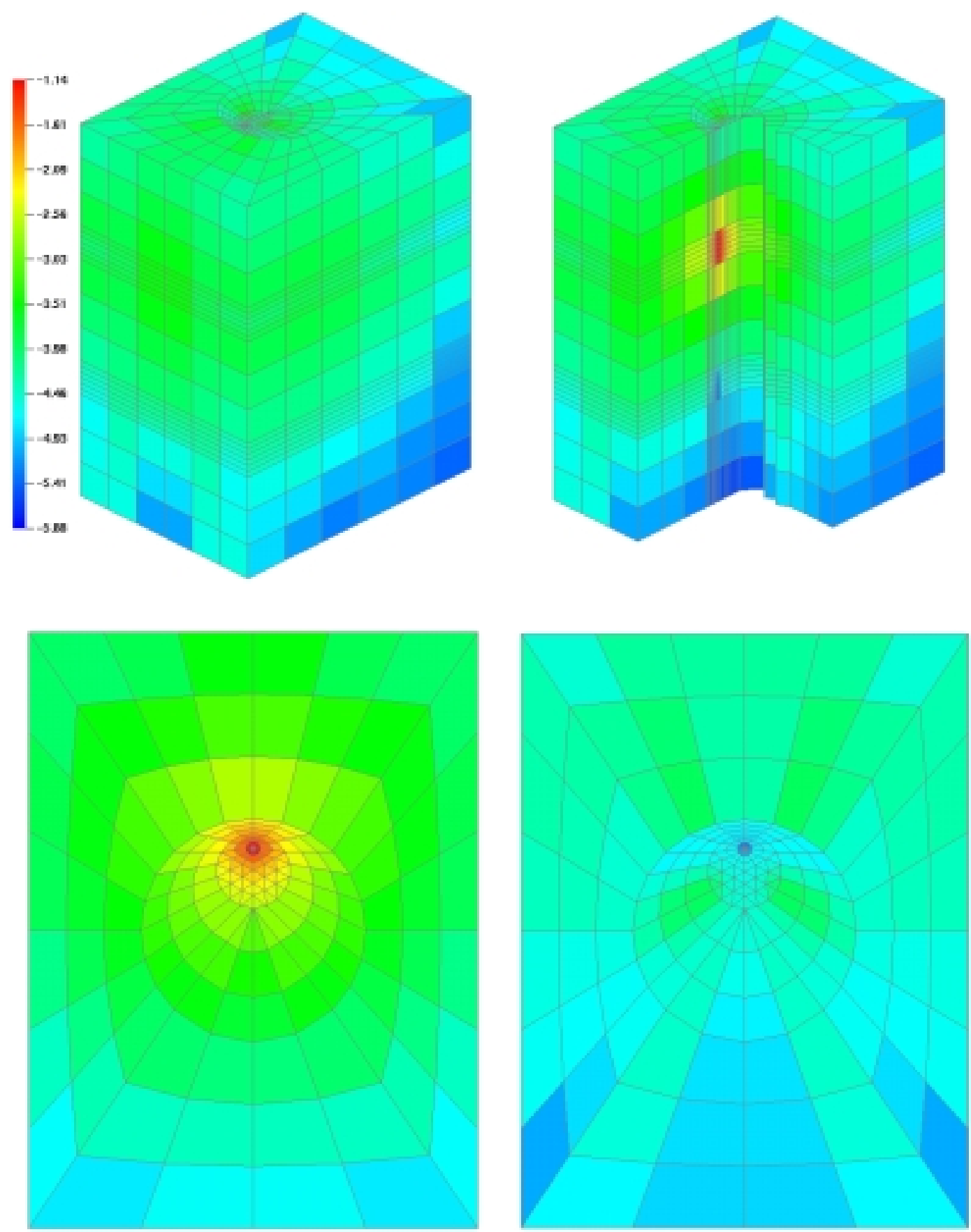

Figure 24. SZCB logarithmic solution for the circles-in-circles problem. 
As we expect, the solution is the largest in the source region and quickly diminishes as we move away from this region. We also notice that the strongly absorbing region causes the solution to drop rapidly. The solution from both discretizations has the general shape that we would expect and the magnitudes of both solutions compare favorably. Unfortunately it also appears that both schemes suffer from ray effects even though we are using 80 angles in this problem. These ray effects manifest themselves as rays where the solution is larger than in the surrounding cells when there is no physical reason for this to occur.

To further compare the solutions that these two schemes produce we have plotted in Figure 25 the solution as a function of z-position through the inner cylinder. Again the results are just as we expect. The solution is the largest in the region that that contains the source and decreases as we travel away from the source. There is a significant depression in the solution in the strongly absorbing region. There are two solutions for each spatial discretization. The line in this chart represents a refined solution with $70 \mathrm{z}$-levels. The data points represent the 21-layer solution. What we notice is that the refined solutions are very close to one another whereas the coarse solutions are not. This is a direct repercussion of the lower order of accuracy for UCBL when compared to SZCB.

The results of this circles-in-circles problem show that the UCBL and SZCB discretizations behave very similarly and produce solutions that are very close to each other. The UCBL solution appears to transport particles in the z-direction through the optically thin inner cylinder more effectively than the SZCB solution. This is a result of the characteristic-like features built into the UCBL scheme while the SZCB solution tends to be more numerically diffusive and so transmits fewer particles in the z-direction. 


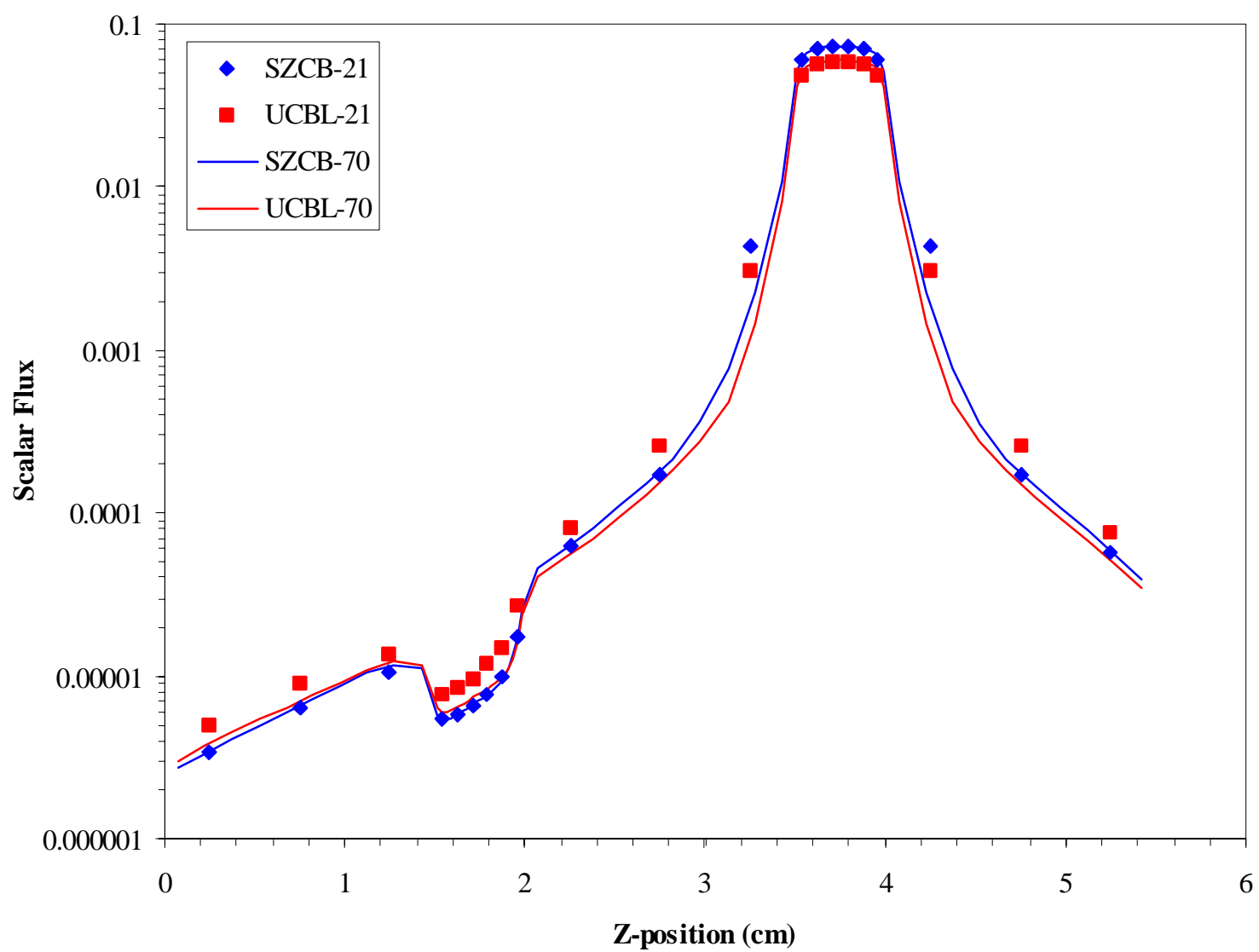

Figure 25. Line-outs at $(x, y)=(2.56,1.47) \mathrm{cm}$ of the UCBL and SZCB solutions as a function of z-position.

\section{G. MOX Test Assembly in the ATR}

Up to this point we have shown numerical results from some specific test cases. Most of these have been small problems that show general code capability and accuracy of the 3D UCBL scheme. Now we are interested in focusing our attention on a real-world problem. We want to model the Advanced Test Reactor Mixed Oxide ( $\mathrm{MOX}$ - a mixture of $\mathrm{UO}_{2}$ and $\mathrm{PuO}_{2}$ ) test assembly geometry to demonstrate the abilities of our scheme to model a complex spatial grid combined with detailed material properties.

This problem consists of a column of water (with dissolved Beryllium) with the cylindrical MOX test assembly set off-center as shown in Figures 26 - 28. The test assembly consists of three MOX fuel cylinders and three cooling tubes encased in an aluminum frame. In the vertical direction there are layers of steel and beryllium that separate three sections of fuel. The top and bottom endcaps are aluminum. We model only the lower half of the geometry using a reflective boundary condition at the mid-plane to simulate the full geometry. 


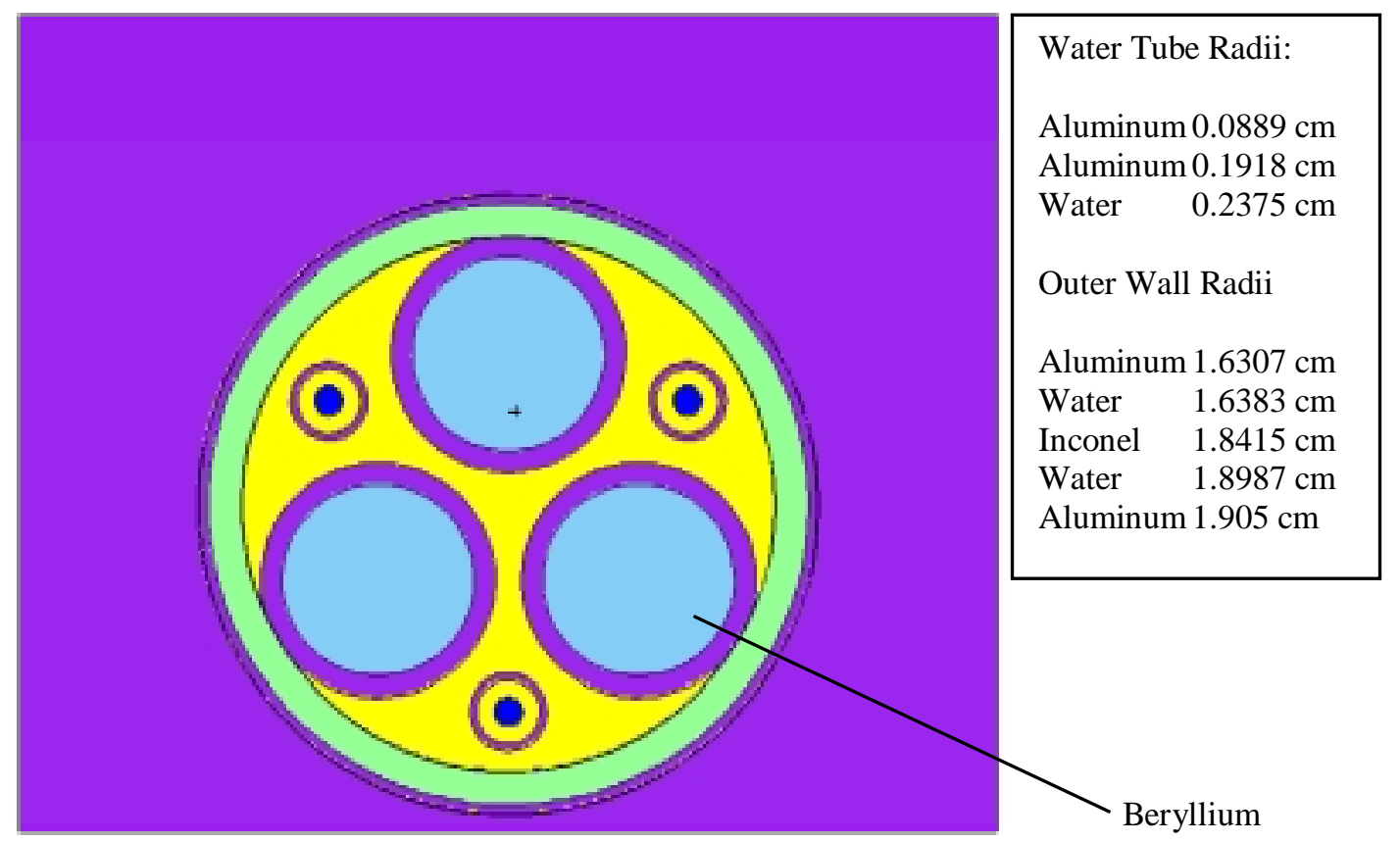

Cross Section at $z=30 \mathrm{~cm}$.

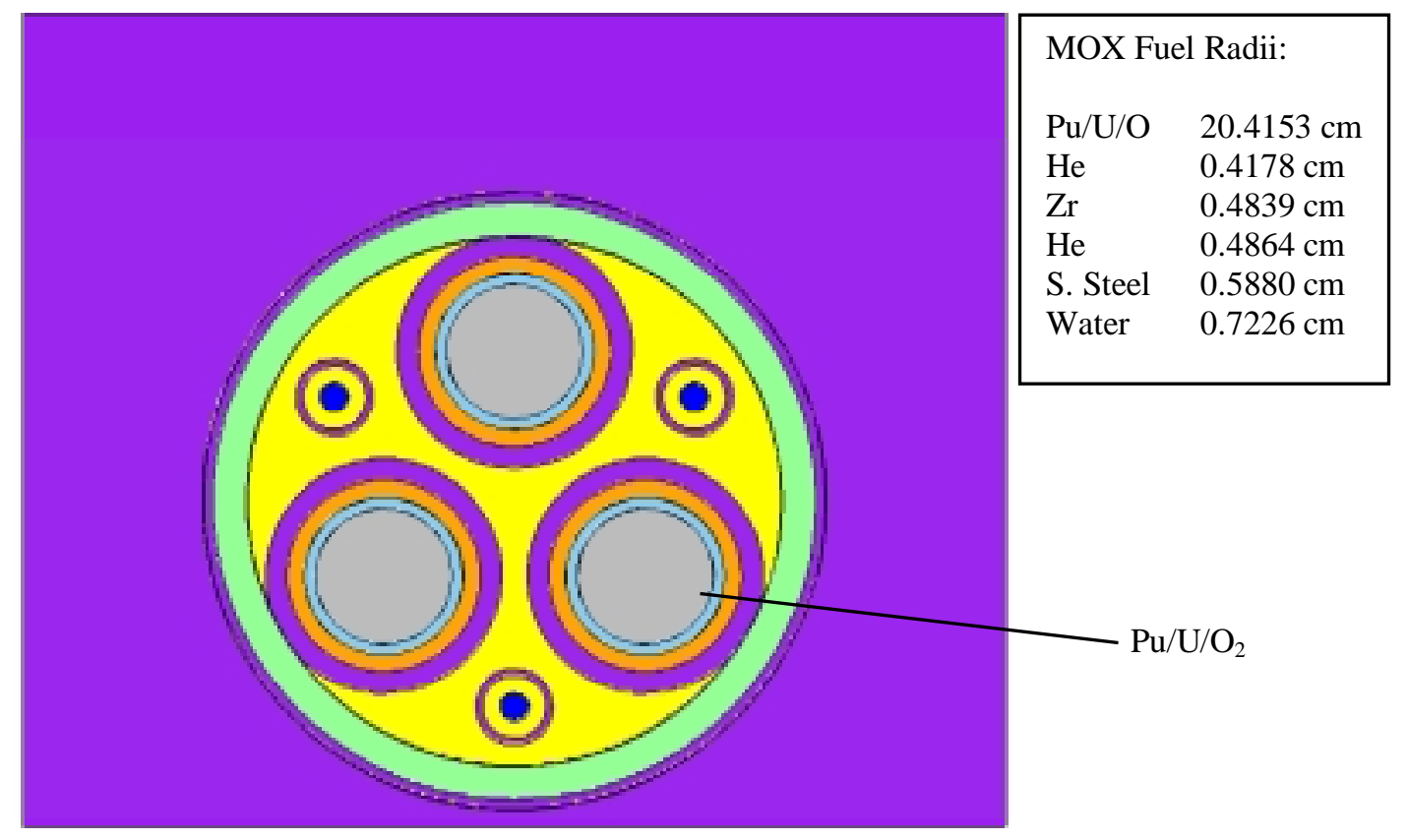

Cross Section at $z=35 \mathrm{~cm}$.

Figure 26. XY cutaways from the MOX assembly geometry at $\mathrm{z}=30$ and $35 \mathrm{~cm}$.. 

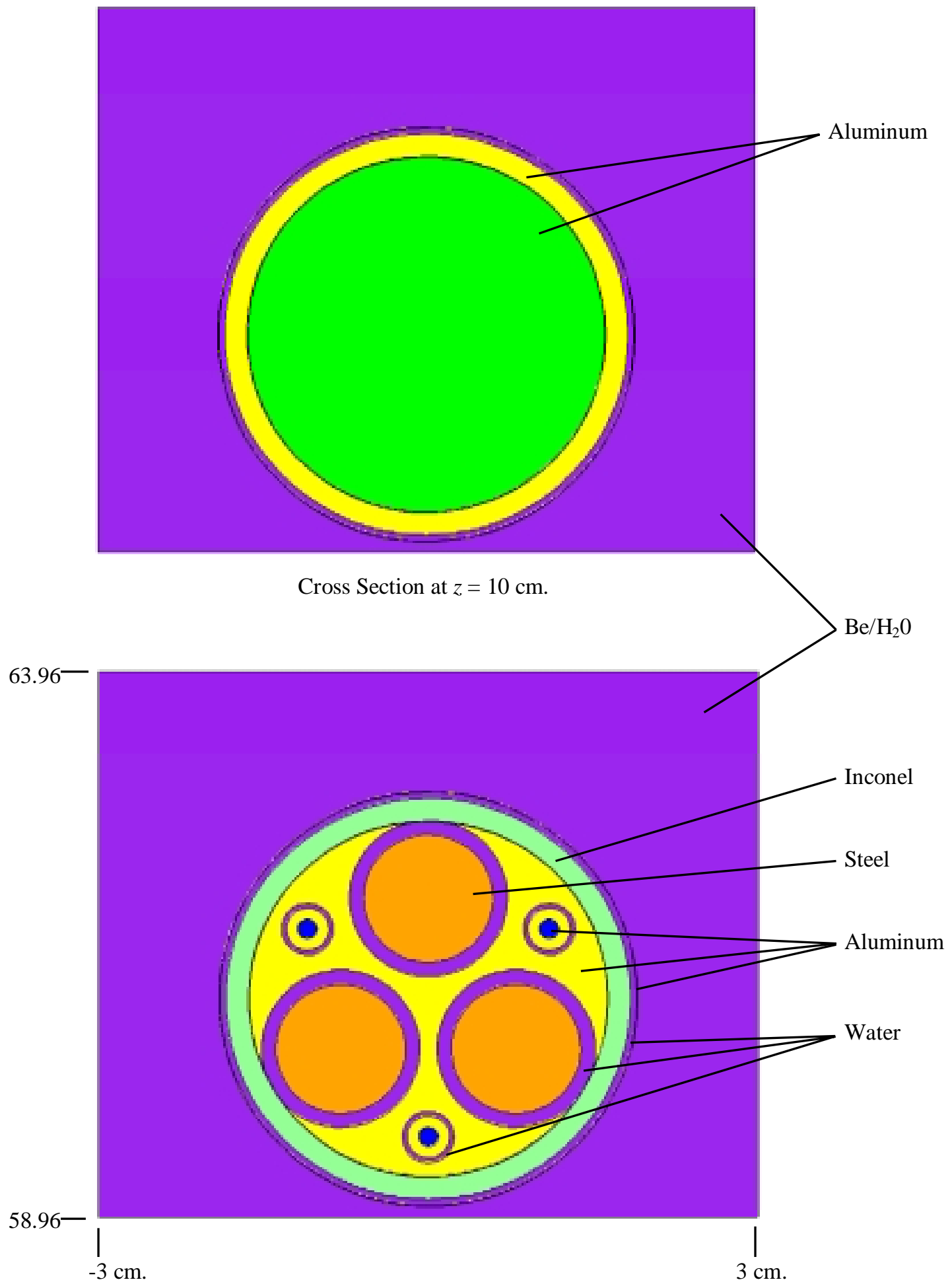

Cross Section at $z=29 \mathrm{~cm}$.

Figure 27. XY cutaways from the MOX assembly geometry at $z=10$ and $29 \mathrm{~cm}$. 


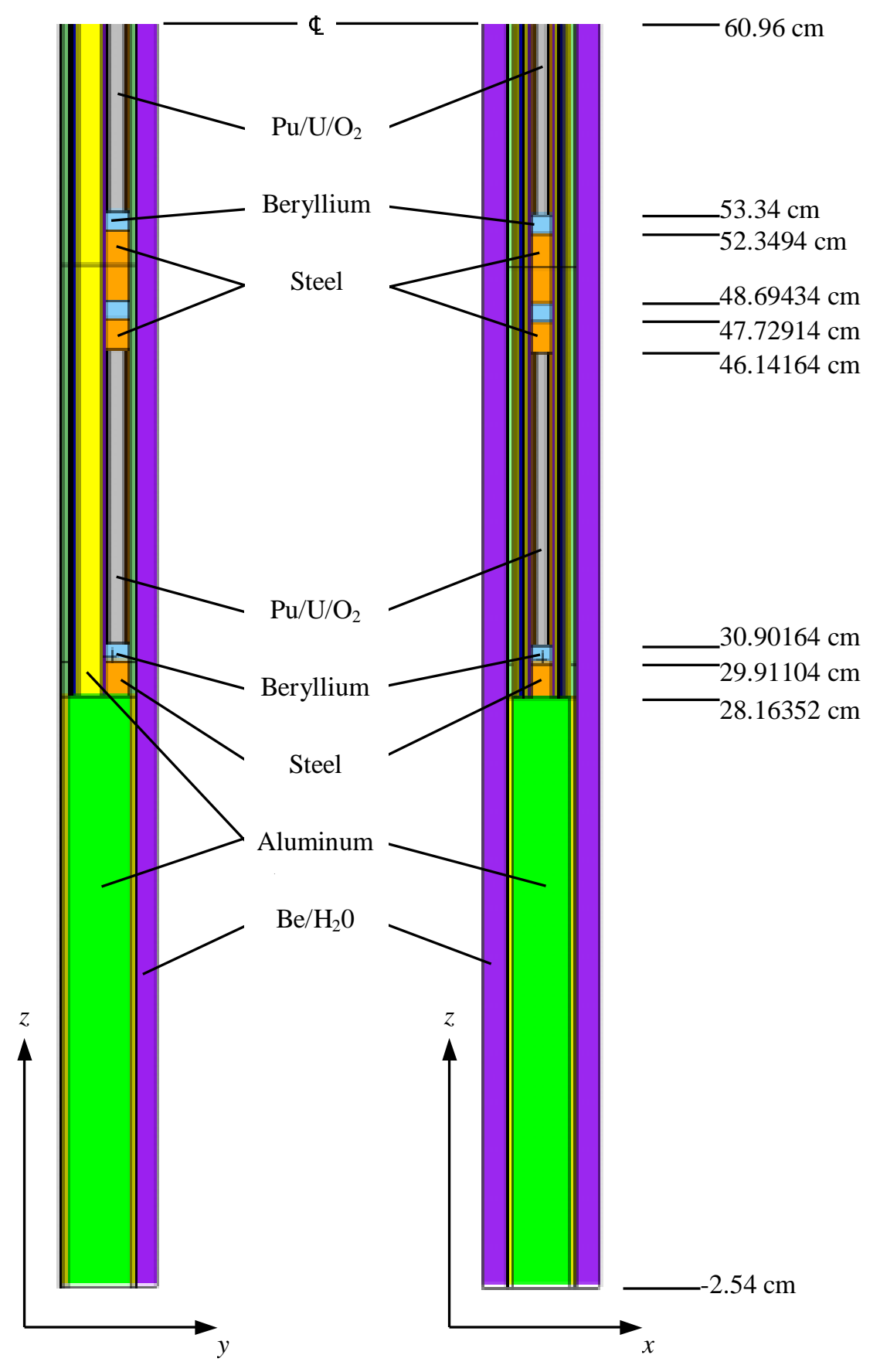

Figure 28. YZ and XZ cutaways of the MOX capsule geometry. 
In Figures 27 and 28 we display four different XY cross sections (at $z=10 \mathrm{~cm}, 29 \mathrm{~cm}, 30 \mathrm{~cm}$ and $35 \mathrm{~cm}$ ). The bottom layer is an aluminum endcap. The actual MOX fuel is in the regions between $30.9-46.1 \mathrm{~cm}$ and $53.3-60.96 \mathrm{~cm}$. There are three cylindrical MOX assemblies with $120^{\circ}$ rotational symmetry inside of the main aluminum structure. The column of MOX fuel is immediately surrounded by a helium gap, a layer of beryllium, another helium gap and then encased in a stainless steel sheath. This whole assembly is separated from the aluminum super structure by a layer of water. The dimensions of each of these columns are shown in Figure 28. The assembly also has three water ports with $120^{\circ}$ rotational symmetry that are between each of the MOX fuel assemblies. These ports consist of a two-layer aluminum rod surrounded by water. The dimensions of these ports are also shown in Figure 28. There are two additional layers that exist at both ends of the fuel. The layer next to the endcap is a stainless steel block. Sandwiched between this steel block and the MOX fuel is a layer of beryllium. Above the first layer of fuel there are four layers of steel and beryllium that separate the lower fuel region from the middle fuel region. From bottom to top these layers are stainless steel, beryllium, stainless steel and beryllium. The appropriate (matching materials) cross sectional view from Figures 27 and 28 apply to these four regions.

We have modeled this problem using a SMaRT-Z grid. ${ }^{26}$ A SMaRT-Z grid is a mixed quadrilateral and triangular cell grid in the XY plane and regular in the $z$-direction. Our grid has 1170 quadrilateral and triangular zones in the $\mathrm{XY}$ plane and $21 z$-levels. In this simulation we use an $\mathrm{S}_{6}$ level-symmetric quadrature set and the two-group isotropic scattering cross sections shown in Table IV.

Table V

ATR MOX Assembly Cross Sections

\begin{tabular}{|c|c|c|c|c|c|}
\hline Material & $\sigma_{\mathrm{t}}\left(\mathrm{cm}^{-1}\right)$ & \multicolumn{2}{|c|}{$\sigma_{\mathrm{s}}\left(\mathrm{cm}^{-1}\right)$} & \multicolumn{2}{|c|}{$v \sigma_{\mathrm{f}}\left(\mathrm{cm}^{-1}\right)$} \\
\hline $\mathrm{He}$ & 0.0001 & 0.00008 & 0.00001 & & \\
\hline & 0.0001 & & 0.00008 & & \\
\hline $\mathrm{Be}$ & 0.35 & 0.10 & 0.20 & & \\
\hline & 0.60 & & 0.55 & & \\
\hline $\mathrm{Zr}$ & 0.35 & 0.25 & 0.08 & & \\
\hline & 0.70 & & 0.65 & & \\
\hline $\mathrm{Al}$ & 0.03 & 0.005 & 0.02 & & \\
\hline & 0.06 & & 0.05 & & \\
\hline Water & 1.10 & 0.10 & 0.9 & & \\
\hline & 2.20 & & 2.0 & & \\
\hline Inconel & 0.60 & 0.1 & 0.4 & & \\
\hline & 1.30 & & 1.0 & & \\
\hline Stainless Steel & 0.35 & 0.03 & 0.30 & & \\
\hline & 0.70 & & 0.65 & & \\
\hline $\mathrm{PuUO}_{2}$ & 0.10 & 0.03 & 0.01 & 0.50 & 0.10 \\
\hline & 1.70 & & 1.30 & 1.20 & 0.15 \\
\hline
\end{tabular}


The SMaRT-Z mesh layout and zone-averaged UCBL solution for the two groups are shown in Figures 29 and 30. In each of these figures we show three views. On the left is a perspective view that allows us to notice flux variations in the z-direction. The top right view shows the solution at the top of the assembly and the bottom right view shows the solution at the bottom of the assembly. The same 2D mesh is used for each z-level but the material properties for each cell in each z-level vary to simulate the actual geometry as closely as possible.

We notice that the simulation produces a reasonable solution for the spatial discretization used. The solution shown in this figure has been normalized so that the range of the scalar flux is $[0,1]$. The maximum flux in group 1 is $37.2 \%$ of the maximum flux value for group 2 . In this particular simulation we implemented an isotropic incident angular boundary source on the maximum $y$-surface for both energy groups. This boundary condition simulates the incident current from the Advanced Test Reactor, which is several $\mathrm{cm}$ away from the test assembly we have simulated. We notice that for both groups the solution is largest at the incident face. For group one the flux peaks in the MOX fuel elements as expected. We also notice that the absorption is stronger for group 1 in the capsule regions and this is reflected in the lower solution values for group one in the non-fissile regions of the capsule. For both groups the leakage out of the bottom of the assembly is evident by the lower flux values in the bottom z-level. In the fast group, the slightly cooler z-levels are due to the absence of fuel. The flux profile shown in these figures is a reasonable approximation of what we expect for the MOX fuel assembly. By running this problem we have demonstrated that our scheme can easily simulate real-world problems that have complex geometries with varying material properties. 


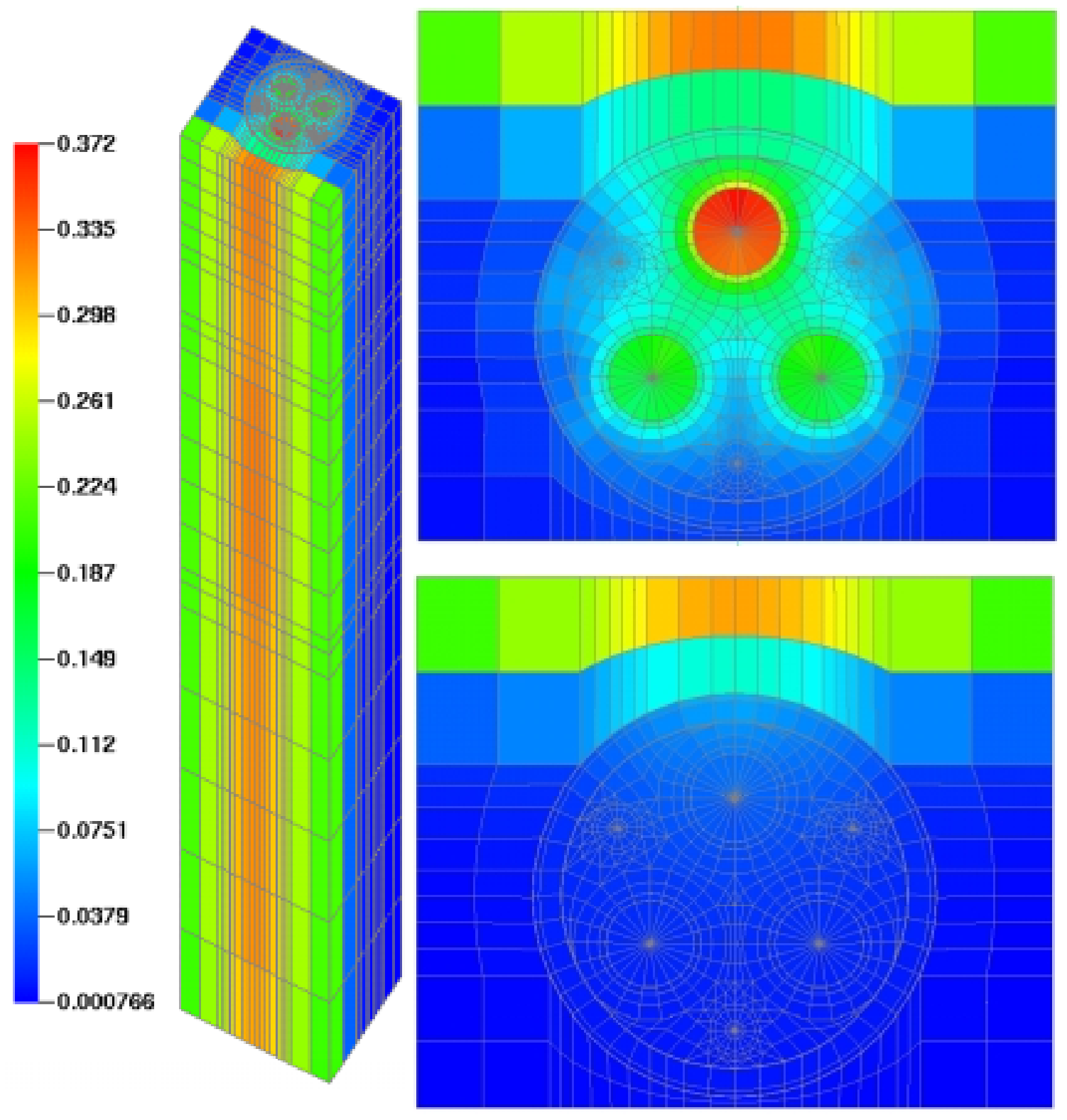

Figure 29. UCBL Group 1 Solution for the ATR MOX Assembly Simulation. 


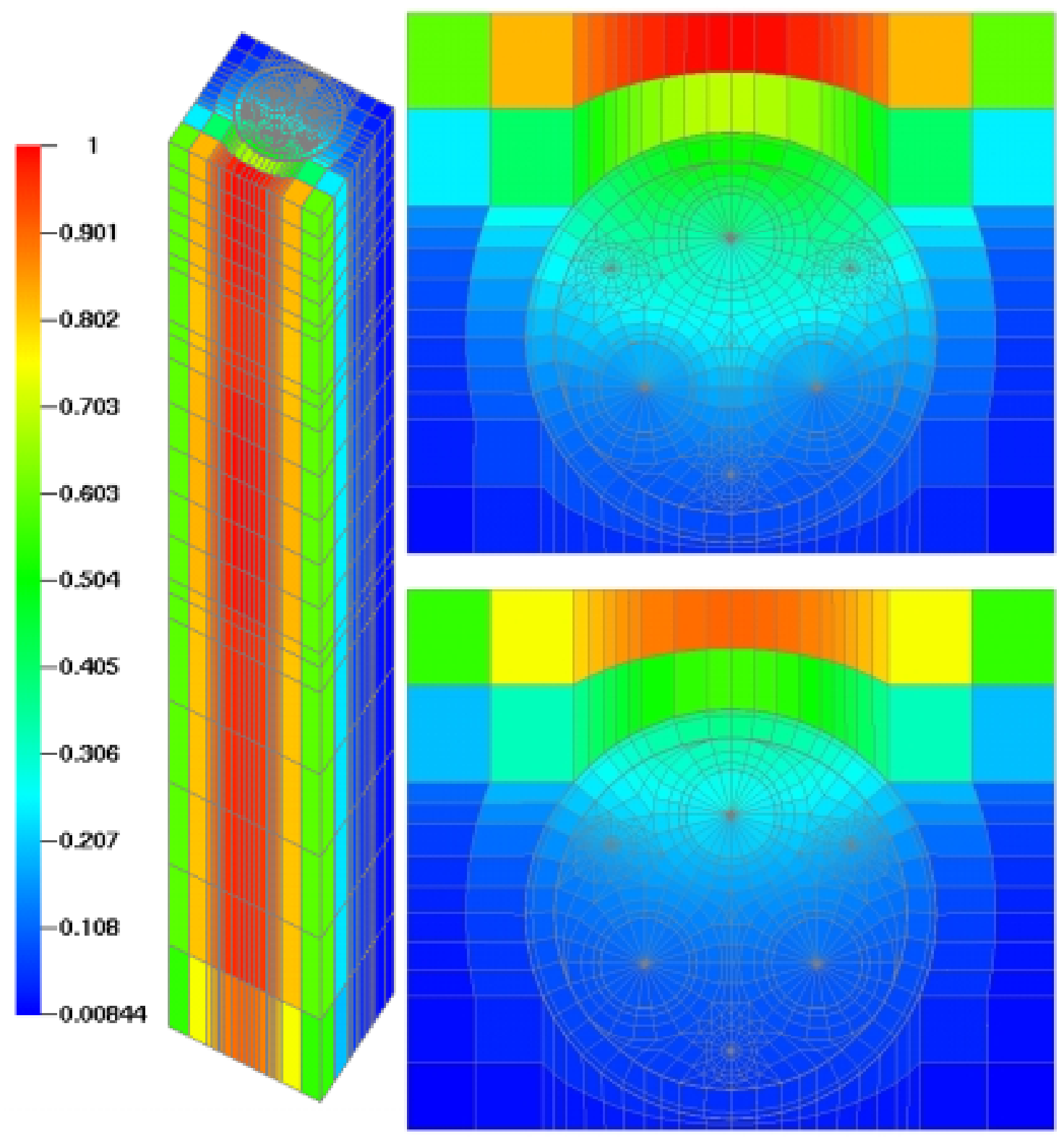

Figure 30. UCBL Group 2 Solution for the ATR MOX Assembly Simulation. 


\section{H. Summary of Numerical Results}

In this chapter we have solved a wide variety of problems using the 3D UCBL spatial discretization scheme. In most cases we have been able to compare our solution with analytic solutions or with the solutions of established codes and discretizations. Through these comparisons we have shown that 3D UCBL solutions demonstrate all of the features that we designed it to capture (see Chapter I for a list of features) with the exception of second-order accuracy.

We have shown 3D UCBL can utilize unstructured meshes consisting of mixed cell shapes and that it works for both eigenvalue and fixed source calculations. We have demonstrated performance for multiregion and mixed material problems with multi-group and anisotropic scattering cross sections. UCBL does remarkably well in avoiding negative solutions and minimizing numerical diffusion. Unfortunately these later two points come at the expense of limiting our scheme to first-order accuracy. Additionally, we have shown deficiencies in the present reflective boundary algorithm and have noticed that $3 \mathrm{D}$ UCBL is expensive computationally. We have shown that our scheme compares favorably with accepted discretizations and permits a great deal of flexibility in the mesh that may be used to model a problem. Even though our analysis in Chapter III shows that our scheme should do an excellent job of being both accurate and robust in optically thick and diffusive regimes, we were unable to demonstrate this feature because we lacked the computational resources to produce numerical results without synthetic acceleration. 


\section{CHAPTER V}

\section{CONCLUSIONS}

\section{A. Summary}

We have developed the new Upstream Corner Balance Linear, UCBL, spatial discretization for 3D neutronics and radiative transport problems. This scheme has its origins in 2D Upstream Corner Balance, UCB, methods. 3D UCBL was designed for use on grids of arbitrary polyhedra in much the same way that the 2D UCB scheme and its predecessor, the 2D Simple Corner Balance scheme, were designed for use on arbitrary polygonal grids. All of these corner balance methods impose conservation ("balance") on corners. These corners are subcell polyhedra in our 3D scheme and are broken down into tetrahedral wedges to simplify our sweeping algorithm by eliminating reentrant surfaces. The 3D upstream scheme developed here has a lower-triangular matrix allowing us to sweep the spatial mesh once subcell at a time without inverting any matrices. This makes the scheme much easier to implement for truly arbitrary grids and makes the algorithm computationally less expensive to run.

We made extensive use of asymptotic analysis to develop the 3D UCBL discretization. The two primary design tasks were to create a scheme that utilized an arbitrary polyhedral mesh and to ensure that our scheme obtains an accurate solution in the diffusion limit. Our scheme is designed to limit to Palmer's vertex-centered discrete diffusion solution in thick diffusive regions. Because of this limit we expect UCBL to have excellent behavior in the thick and diffusive regimes found in radiative transfer problems. For example, UCBL should obtain the exact solution to leading order in a diffusive region whose analytic diffusion solution is a linear function, regardless of the distortion of the spatial grid. The only concern in diffusive problems is that the scheme's accuracy may be reduced given unresolved boundary layers since the leading-order solution satisfies a Marshak-weighted boundary condition. That is, although the interior solution may have the correct shape, it may approach an inaccurate value on the boundary. This flaw is important only when the intensity incident upon a diffusive region is significantly anisotropic and the region has sufficiently little absorption. We were unable to test our theoretical predictions about UCBL's behavior in diffusive regions because there does not yet exist an iterative method for UCBL that can solve diffusive problems in a reasonable amount of time. We address this in more detail below in our discussion of future work.

There were other good properties that we attempted to instill in our scheme. We specifically created an algebraically linear discretization that yields strictly non-negative solutions in source-free purely absorbing media. The numerical approximations were also designed to minimize the numerical diffusion of a beam in a vacuum. While we attempted to minimize truncation error, other higher-priority requirements forced the 
scheme to have first-order truncation error. As Godunov has shown ${ }^{23}$ this limitation is a result of requiring the scheme to be both monotonic and algebraically linear. An exception is that in problems that are nearly one-dimensional, UCBL can exhibit second-order behavior.

We have tested the 3D UCBL scheme on a variety of problems including fixed source and eigenvalue problems. We demonstrated accuracy in problems with anisotropic scattering, multiple materials, multigroup cross sections and a variety of geometries and boundary conditions. Unfortunately we were unable to test our scheme on a completely arbitrary grid containing many different polyhedral cell types, because such a grid was not available for our use. However, we did test the scheme using grids containing distorted hexahedral cells, triangular prisms, and other degenerate hexahedral cells. In each test the UCBL scheme performed as our various analyses had predicted, and in regimes that we could not analyze it performed as we expected based upon the performance of its predecessor, 2D UCB. For example, tests showed that UCBL does remarkably well in coarsely discretized problems with steep solution gradients. We ran two test problems with complicated geometric structures that could not be efficiently modeled with orthogonal grids. In the fist of these we solved a test problem inspired by nuclear well logging, using both the new 3D UCBL and Richardson's 3D CB scheme. In this problem the two methods should produce similar results, and they did. In another test problem, we simulated a MOX fuel assembly that is being irradiated in the reflector region of the Advanced Test Reactor at the Idaho National Environmental Engineering Laboratory. This demonstrated UCBL's ability to calculate a real-world problem with complex geometry and mixed materials.

\section{B. Recommendations for Future Work}

During the course of this work, we encountered issues that were beyond the scope of this work but that warrant further investigation. We discuss these below.

1) Truncation Error: Because UCBL is monotonic and algebraically for source-free purely absorbing media it is limited according to Godunov's theorem to have first-order truncation error. We note that $2 \mathrm{D} \mathrm{UCB}$ is not limited by this proof because it is not a monotonic method. We believe that we can modify the UCBL $f e z$ closure to mimic the behavior of 2D UCB's corner-to-corner closure and gain second-order accuracy. In doing so, we would lose the nice property of monotonicity and would introduce unphysical oscillations in some streaming problems. This trade-off may be a net gain and should be investigated.

2) Nonlinear Scheme: It may be possible to devise a UCB scheme that is second-order, monotonic, and possesses the most important good properties of our UCBL scheme if we remove the restriction of algebraic linearity. Several spatial discretizations introduced recently have achieved remarkable accuracy be clever use of nonlinear approximations. ${ }^{27-30}$ The application of those ideas to arbitrary grids could be quite fruitful and should be explored. 
3) Excessive Storage: The excessive storage requirements required by our wedge-based scheme are a concern: a simple hexahedral cell contains 48 wedges. We believe that we can significantly reduce storage requirements by returning to a truly corner-based algorithm. This is not a huge step since we can utilize what is known about the wedge-based closures of UCBL, the corner-based SZCB algorithm and 2 UCB to create a consistent corner based scheme for unstructured polyhedral grids. In Appendix $\mathrm{C}$ we consider one possible development of such a method but we have not done any extensive testing with this scheme. The discretization developed Appendix $\mathrm{C}$ also addresses item (1) of this list.

4) Reflective Boundary Algorithm: In Chapter IV we discuss the development, use, and problems found with the reflective boundary algorithm developed in Appendix B. Under the time restrictions of this work we were unable to develop an algorithm that is robust and preserves multiple angular moments of the angular flux. Current work is being done at Los Alamos National Laboratory to address this issue. ${ }^{31}$

5) Acceleration for Diffusive Problems: There is certainly a need for some type of iterative acceleration to reduce the iteration count in thick and diffusive regions. Zika and Nowak have implemented a specialized version of transport synthetic acceleration, TSA in their code. ${ }^{32-33}$ An alternate approach would be to use diffusion synthetic acceleration, DSA. It may be possible to use Palmer's diffusion discretization to accelerate UCBL; there is reason to hope that such an approach could work since 3D UCBL limits to Palmer's solution in thick diffusive regions. There is no guarantee that such an approach would be successful, but it appears to be a possibility worth investigating. 


\section{REFERENCES}

1. E. E. LEWIS and W. F. MILLER, Jr., Computational Methods of Neutron Transport, American Nuclear Society, Inc., La Grange Park, IL (1993).

2. J. J. DUDERSTADT and L. J. HAMILTON, Nuclear Reactor Analysis, John Wiley \& Sons, New York, NY (1976).

3. J. M. MCGHEE, R. M. ROBERTS and J. E. MOREL, "Dante Boltzmann Transport Solver: An Unstructured Mesh, 3-D Spherical Harmonics Algorithm/Compatible with Parallel Computer Architecture," LA-UR-97-1031, Los Alamos National Laboratory (1997).

4. E. E. LEWIS, "Progress in Multidimensional Neutron Transport Computation," Nucl. Sci. Eng., 64, 279 (1977).

5. T. R. HILL and R. R. PATERNOSTER, "Two-Dimensional Spatial-Discretization Methods on a Lagrangian Mesh,” LA-UR-82-1055, Los Alamos National Laboratory (1982).

6. D. S. MILLER, D. E. BUTON, "3D Mesh Optimization Methods for Unstructured Polyhedra: A Progress Report,” Lawrence Livermore National Laboratory, UCRL-JC-119206 (1994).

7. J. E. MOREL, J. M. MCGHEE and W. F. WALTERS, "3-D Research Transport Codes at Los Alamos," LA-UR-94-3383, Los Alamos National Laboratory (1994).

8. T. A. WAREING, J. M. MCGHEE and J. E. MOREL, "ATILLA: A Three-Dimensional, Unstructured Tetrahedral Mesh Discrete Ordinates Transport Code,” Trans. Am. Nucl. Soc., 75, 146 (1996).

9. T. S. PALMER, “A Point-Centered Diffusion Differencing for Unstructured Meshes in 3-D, ” Proc. Int. Conf. Mathematics and Computations, Reactor Physics, and Environmental Analyses, Portland, OR, April 30 - May 4, 1995, Vol. 2, p. 897, American Nuclear Society (1995).

10. M. L. ADAMS, “A New Transport Discretization Scheme for Arbitrary Spatial Meshes in XY Geometry," Proc. Int. Conf. Advances in Mathematics, Computations, and Reactor Physics, Pittsburgh, PA, April 28 - May 2, 1991, Vol. 3, p. 13.2 2-1, American Nuclear Society (1991).

11. M. L. ADAMS, "Subcell Balance Methods for Radiative Transfer on Arbitrary Grids," Transp. Theory Stat. Phys., 26, Nos. 4\&5, 385 (1997).

12. E. W. LARSEN and J. E. MOREL, "Asymptotic Solutions of Numerical Transport Problems in Optically Thick Diffusive Regimes II," J. Comp. Phys., 83, 212 (1989). See also "Corrigendum,” J. Comp. Phys., 91, p. 246 (1990).

13. E. W. LARSEN, "On Numerical Solutions of Transport Problems in the Diffusion Limit," Nucl. Sci. Eng., 83, 90 (1983).

14. K. A. MATHEWS, "On the Propagation of Rays in Discrete Ordinates," Nucl. Sci. Eng., 132, 155 (1999).

15. R. L. RICHARDSON, "A Three Dimensional Corner Balance Method for Spatial Discretization of the Transport Equation,” M.S. Thesis, Texas A\&M University, College Station, TX (1994).

16. G. C. POMRANING, "Radiation Hydrodynamics - Notes from a short course given at Los Alamos," LA-UR-82-2625, Los Alamos National Laboratory (1982). 
17. R. J. LeVEQUE, D. MIHALAS, E. A. DORFI and E. MÜLLER, Computational Methods for Astrophysical Fluid Flow, Springer-Verlag, Berlin, Germany (1998). See section entitled "Radiation Hydrodynamics".

18. F. MALGAVI and G. C. POMRANING, "Initial and Boundary Conditions for Diffusive Linear Transport Problems,” J. Math. Physics, 32, 805 (1990).

19. S. CHANDRASEKHAR, Radiative Transfer, Dover, NY (1960).

20. SNAC ( $\mathrm{S}_{\mathrm{N}}$ transport on Arbitrarily Connected grids) is a computer code originally developed at Lawrence Livermore National Laboratory by M. L. Adams. Its purpose is to serve as a testbed for deterministic transport methods, especially those designed for arbitrary grids.

21.E. W. LARSEN, "The Asymptotic Diffusion Limit of Discretized Transport Problems," Nucl. Sci. Eng., 112, 336 (1992).

22. R. S. BAKER and R. E. ALCOUFFE, "Time-Dependent 3-D Deterministic Transport on Parallel Architectures using DANTSYS/MPI,” LA-UR-95-4507, Los Alamos National Laboratory (1995).

23. S. K. GODUNOV, "Difference Methods for Numerical Calculations of Discontinuous Solutions of Hydrodynamic Equations,” Matematicheskii, Sbornik, 47, No. 3, 271 (1959).

24. K. D. LATHROP and A. LEONARD, "Comparisons of Exact and $\mathrm{S}_{\mathrm{N}}$ Solutions of the Monoenergetic Critical Equation with Anisotropic Scattering," Nucl. Sci. Eng., 22, 115 (1965).

25. J. DAHL, Los Alamos National Laboratory, Private Communication (1999).

26. SMaRT is a computer code originally developed at Lawrence Livermore National Laboratory by D. Nielsen, Jr., Its purpose is to generate 2-D unstructured meshes of triangles and quadrilaterals.

27. K. A. MATHEWS and C. R. BRENNAN, "Exponential Characteristics Nonlinear Radiation Transport Method for Unstructured Grids of Triangular Cells," Nucl. Sci. and Eng., 126, 264-281 (1997).

28. K. A. MATHEWS, G. SJODEN, and B. M. MINOR, "Exponential Characteristic Spatial Quadrature for Discrete Ordinates Radiation Transport in Slab Geometry," Nucl. Sci. and Eng., 118, $24-37$ (1994).

29. K. A. MATHEWS and B. M MINOR, "Adaptive Characteristic Spatial Quadratures for Discrete Ordinates Neutral Particle Transport - The Rectangular Case," Trans. Theory Stat. Phys., 22, 5, 655 (1993).

30. T. A. WAREING and W. F. Walters, "An Exponential Discontinuous Scheme for X-Y Geometry Transport Problems," Trans. Am. Nucl. Soc., Philadelphia, PA 72 (1995).

31. J. E. MOREL, Los Alamos National Laboratory, Private Communication (1999).

32. M. ZIKA, Lawrence Livermore National Laboratory, Private Communication (1999).

33. G. L. RAMONE and M. L. ADAMS, "A Transport Synthetic Acceleration Method for Transport Iterations," Nucl. Sci. Eng., 125, 257 (1997). 


\section{APPENDIX A}

\section{CORNER-AVERAGED SCALAR FLUXES ABOUT A VERTEX ARE EQUAL}

In the development of our closure equation we have stated that our FEP step closure produces a dual grid where all the leading-order corner-averaged scalar fluxes about a common vertex are equal. In this appendix we show that this is true.

\section{A. 1. Background}

In Chapter II we started with the $O(1)$ wedge pseudo-balance equation. After integrating over all angles and noting that to leading order the $f e z, p e z$ and $f p z$ closures are isotropic and that in the interior of the problem the fep is half range isotropic to leading order we arrive at the following expression.

$$
\sum_{w \in C}\left(\phi_{c}^{(0)}-\phi_{\tilde{c}_{f e p}}^{(0)}\right) \vec{A}_{f e p, w} \cdot \sum_{\vec{A}_{f e p, w} \cdot \bar{\Omega}_{m}>0} w_{m} \vec{\Omega}_{m}=0 .
$$

We have stated that this equation implies that all the leading order corner averaged fluxes about a common vertex are equal in magnitude.

$$
\phi_{c}^{(0)}=\phi_{\tilde{c}_{f e p}}^{(0)} \equiv \phi_{p}^{(0)} \quad \forall c, \tilde{c} \in p .
$$

We will examine the validity of this statement below.

\section{A. 2. Proof}

We start our proof by defining some terms that will simplify our analysis. Note first that each surface separating two corners is constructed from two fep wedge faces.

$$
\sum_{f p \in c}\left(\phi_{c}^{(0)}-\phi_{\tilde{c}_{f p}}^{(0)}\right)\left[\sum_{\bar{A}_{f e p, w 1} \cdot \vec{\Omega}_{m}>0} w_{m}\left(\vec{A}_{f e p, w 1} \cdot \vec{\Omega}_{m}\right)+\sum_{\vec{A}_{f e p, w 2} \cdot \bar{\Omega}_{m}>0} w_{m}\left(\vec{A}_{f e p, w 2} \cdot \vec{\Omega}_{m}\right)\right]=0 .
$$

The notation $f p \in c$ means all of the corner faces defined by $f$ and $p$ for the current corner. For a brick mesh each corner will have three $f p$ faces (see Figure 1). Each $f p$ face will consist of two wedge faces $f e p, w 1$ and fep,w2. We will assume that our quadrature set only has positive weights so that we are guaranteed that each sum will be strictly positive. We have also used the notation $\tilde{c}_{f p}$ to represent the corner that shares the $f p$ face with the current corner.

To simplify our equation we define a new parameter $a_{i j}$. The index $i$ represents the corner we are examining and the index $j$ represents all corners that that share vertex $p$ with corner $i\left(j=1 \ldots N_{c}\right)$. Note that $a_{i j}$ will be zero for any two corners that do not share a face.

$$
a_{i j} \equiv \sum_{\bar{A}_{f e p, w 1} \cdot \bar{\Omega}_{m}>0} w_{m}\left(\vec{A}_{f e p, w 1} \cdot \vec{\Omega}_{m}\right)+\sum_{\bar{A}_{f e p, w 2} \cdot \bar{\Omega}_{m}>0} w_{m}\left(\vec{A}_{f e p, w 2} \cdot \vec{\Omega}_{m}\right) .
$$


The two area vectors represent outward normal vectors for the two wedges that form the corner-corner interface and are oriented away from corner $i$ and into corner $j$. If we examine $a_{i j}$ for each corner intersecting vertex $p\left(i=1 \ldots N_{c}\right)$ we note the following properties:

1. $a_{i j}=0$ for all corners $i$ and $j$ that do not share a common face.

2. $a_{i i}=0$.

3. $\quad a_{i j}>0$ for all corners $i$ and $j$ that do share a common face.

Using these definitions we can write equation (121) as

$$
\sum_{j=1}^{N_{c}} a_{i j}\left(\phi_{i}^{(0)}-\phi_{j}^{(0)}\right)=0, \quad i=1 \ldots N_{c} .
$$

Note that we have also changed to the $(i, j)$ index notation for the leading-order scalar fluxes. Remember that we sum over $j=1 \ldots N_{c}$ but this difference between corner fluxes only shows up when the two corners are adjacent because in the other cases $a_{i j}$ will be zero. We also note that this equation may be written for any interior corner that intersects the vertex $p\left(i=1 \ldots N_{c}\right)$.

We can define another new variable, $m_{i j}$.

$$
\begin{aligned}
& m_{i i} \equiv \sum_{j=1}^{N_{c}} a_{i j}, \\
& m_{i j} \equiv-a_{i j}, \quad i \neq j .
\end{aligned}
$$

We note that $m_{i i}>0$ since all $a_{i j}>0$ and corner $i$ will always share at least three faces with adjacent corners. Using the definition of $m_{i j}$ from equation (124) in equation (123) we can derive another equivalent expression.

$$
\sum_{j=1}^{N_{c}} m_{i j} \phi_{j}^{(0)}=0, \quad i=1 \ldots N_{c} .
$$

We can write this sequence of sums in matrix notation.

$$
\overrightarrow{\vec{F}} \vec{\phi}=0 \text {. }
$$

The matrix $\overrightarrow{\vec{F}}$ is an $N_{c} \mathrm{x} N_{c}$ matrix and the vector $\vec{\phi}$ has $N_{c}$ elements. This matrix is singular since summing all of the rows will yield an empty matrix.

Let us define another variable, $\Delta_{i}$.

$$
\Delta_{i} \equiv \phi_{i}^{(0)}-\phi_{N_{c}}^{(0)} .
$$

We notice the following two properties about $\Delta_{i}$ :

$$
\Delta_{N}=0,
$$

and

$$
\phi_{i}^{(0)}-\phi_{j}^{(0)}=\Delta_{i}-\Delta_{j}
$$

Because of this last relation we can replace the use of $\phi_{j}^{(0)}$ in equations (125) and (126) with $\Delta_{j}$.

$$
\overrightarrow{\vec{F}} \vec{\Delta}=0 \text {. }
$$


We also notice from equation (128) that the $N_{c}^{\text {th }}$ entry of $\vec{\Delta}$ is zero so the $N_{c}^{\text {th }}$ column of $\overrightarrow{\vec{F}}$ adds nothing to the solution. Because this last column adds nothing to the solution it can be removed from the system. We can also remove the last row of $\overrightarrow{\vec{F}}$ because it contains redundant information (i.e. row $N_{c}$ of $\overrightarrow{\vec{F}}$ can be constructed from the other $\left(N_{c^{-}}-1\right)$ rows). We can now write down an $\left(N_{c}-1\right) \mathrm{x}\left(N_{c}-1\right)$ system of equations.

$$
\overrightarrow{\vec{M}} \vec{\delta}=0
$$

If we can show that $\overrightarrow{\vec{M}}$ is symmetric positive definite (SPD) then we know that its inverse exists. If $\overrightarrow{\vec{M}}$ has an inverse then from equation (131) we must require $\vec{\delta}=0$. If this is true then by our definition of $\Delta_{i}$ all leading-order scalar fluxes that share a common vertex must be equal.

We notice the following properties about the elements of matrix $\overrightarrow{\bar{M}}$ :

1. $m_{i j}=m_{j i}$.

2. $m_{i j}<0, i \neq j$.

3. $m_{i i} \geq \sum_{j=1}^{N_{c}-1}\left|m_{i j}\right|$

Property 1 of $m_{i j}$ is a result of property 3 of $a_{i j}$ and equation (124) and means that our matrix is symmetric. Properties 2 and 3 are a result of our definitions of $m_{i j}$ and $a_{i j}$ for off-diagonal terms. Also note that both $i$ and $j$ have the range $1 \ldots\left(N_{c}-1\right)$ so that property 3 will be strictly positive if corner $i$ shares a face with corner $N_{c}$.

We have already stated that the matrix of interest is symmetric. We also now that it contains no complex valued elements so it must also be Hermitian. Since our matrix is Hermitian it will be positive definite if the inner product $\langle\overrightarrow{\vec{M}} \vec{x}, \vec{x}\rangle>0$ holds true for any nonzero $\left(N_{c}-1\right)$ dimensional vector $\vec{x}$. Let us examine this inner product.

$$
\begin{aligned}
\langle\overrightarrow{\vec{M}} \vec{x}, \vec{x}\rangle & =\vec{x}^{T} \overrightarrow{\vec{M}} \vec{x}, \\
& =\sum_{i=1}^{N_{c}-1} x_{i} \sum_{j=1}^{N_{c}-1} m_{i j} x_{j}, \\
& =\sum_{i=1}^{N_{c}-1} m_{i i} x_{i}^{2}+\sum_{i=1}^{N_{c}-1} x_{i} \sum_{\substack{j=1 \\
j \neq i}}^{N_{c}-1} m_{i j} x_{j} .
\end{aligned}
$$

As a result of property 3 of $m_{i j}$ we know that at least for some $m_{i i}$ we will have

$$
m_{i i}>\sum_{\substack{j=1 \\ j \neq i}}^{N_{c}-1}\left|m_{i j}\right| .
$$

The result of equation (133) in equation (132) is that we now have the following inequality.

$$
\langle\overrightarrow{\vec{M}} \vec{x}, \vec{x}\rangle>\sum_{i=1}^{N_{c}-1} \sum_{\substack{j=1 \\ j \neq i}}^{N_{c}-1}\left|m_{i j}\right| x_{i}^{2}+\sum_{i=1}^{N_{c}-1} x_{i} \sum_{\substack{j=1 \\ j \neq i}}^{N_{c}-1} m_{i j} x_{j} .
$$


We continue to develop the right hand side of this inequality by multiplying and dividing the right hand side (RHS) by two.

$$
\sum_{i=1}^{N_{c}-1} \sum_{j=1}^{N_{c}-1}\left|m_{i j}\right| x_{i}^{2}+\sum_{i=1}^{N_{c}-1} x_{i} \sum_{\substack{j=1 \\ j \neq i}}^{N_{c}-1} m_{i j} x_{j}=\frac{1}{2}\left\{\sum_{i=1}^{N_{c}-1} \sum_{\substack{j=1 \\ j \neq i}}^{N_{c}-1}\left|m_{i j}\right| x_{i}^{2}+\sum_{i=1}^{N_{c}-1} \sum_{\substack{j=1 \\ j \neq i}}^{N_{c}-1}\left|m_{i j}\right| x_{i}^{2}+2 \sum_{i=1}^{N_{c}-1} x_{i} \sum_{\substack{j-1 \\ j \neq i}}^{N_{c}-1} m_{i j} x_{j}\right\} .
$$

Now we interchange the indices for the middle term.

$$
\sum_{i=1}^{N_{c}-1} \sum_{j=1}^{N_{c}-1}\left|m_{i j}\right| x_{i}^{2}+\sum_{i=1}^{N_{c}-1} x_{i} \sum_{\substack{j=1 \\ j \neq i}}^{N_{c}-1} m_{i j} x_{j}=\frac{1}{2}\left\{\sum_{i=1}^{N_{c}-1} \sum_{\substack{j=1 \\ j \neq i}}^{N_{c}-1}\left|m_{i j}\right| x_{i}^{2}+\sum_{j=1}^{N_{c}-1} \sum_{\substack{i=1 \\ i \neq j}}^{N_{c}-1}\left|m_{i j}\right| x_{j}^{2}+2 \sum_{i=1}^{N_{c}-1} \sum_{\substack{j=1 \\ j \neq i}}^{N_{c}-1} m_{i j} x_{j} x_{i}\right\} .
$$

We have noted that $m_{i j}$ is symmetric so its indices have not changed. Next we interchange the order in which we complete the summation of term two.

$$
\sum_{i=1}^{N_{c}-1} \sum_{j=1}^{N_{c}-1}\left|m_{i j}\right| x_{i}^{2}+\sum_{i=1}^{N_{c}-1} x_{i} \sum_{\substack{j=1 \\ j \neq i}}^{N_{c}-1} m_{i j} x_{j}=\frac{1}{2}\left\{\sum_{i=1}^{N_{c}-1} \sum_{\substack{j=1 \\ j \neq i}}^{N_{c}-1}\left|m_{i j}\right| x_{i}^{2}+\sum_{i=1}^{N_{c}-1} \sum_{\substack{j=1 \\ j \neq i}}^{N_{c}-1}\left|m_{i j}\right| x_{j}^{2}+2 \sum_{i=1}^{N_{c}-1} \sum_{\substack{j \neq 1 \\ j \neq i}}^{N_{c}-1} m_{i j} x_{j} x_{i}\right\} .
$$

We recognize that we can place an absolute value operator on the $m_{i j}$ in the last term and pull the minus sign outside of the summation.

$$
\sum_{i=1}^{N_{c}-1} \sum_{j=1}^{N_{c}-1}\left|m_{i j}\right| x_{i}^{2}+\sum_{i=1}^{N_{c}-1} x_{i} \sum_{\substack{j=1 \\ j \neq i}}^{N_{c}-1} m_{i j} x_{j}=\frac{1}{2}\left\{\sum_{i=1}^{N_{c}-1} \sum_{\substack{j=1 \\ j \neq i}}^{N_{c}-1}\left|m_{i j}\right| x_{i}^{2}+\sum_{i=1}^{N_{c}-1} \sum_{\substack{j=1 \\ j \neq i}}^{N_{c}-1}\left|m_{i j}\right| x_{j}^{2}-2 \sum_{i=1}^{N_{c}-1} \sum_{\substack{j=1 \\ j \neq i}}^{N_{c}-1}\left|m_{i j}\right| x_{j} x_{i}\right\} .
$$

This process allows us to complete the square of $\left(x_{i}-x_{j}\right)^{2}$ so that we have

$$
\sum_{i=1}^{N_{c}-1} \sum_{j=1}^{N_{c}-1}\left|m_{i j}\right| x_{i}^{2}+\sum_{i=1}^{N_{c}-1} x_{i} \sum_{\substack{j=1 \\ j \neq i}}^{N_{c}-1} m_{i j} x_{j}=\frac{1}{2} \sum_{i=1}^{N_{c}-1} \sum_{\substack{j=1 \\ j \neq i}}^{N_{c}-1}\left|m_{i j}\right|\left(x_{i}-x_{j}\right)^{2},
$$

which means that the inner product is positive and we have confirmed that the matrix is symmetric positive definite.

$$
\langle\overrightarrow{\vec{M}} \vec{x}, \vec{x}\rangle>\frac{1}{2} \sum_{i=1}^{N_{c}-1} \sum_{\substack{j=1 \\ j \neq i}}^{N_{c}-1}\left|m_{i j}\right|\left(x_{i}-x_{j}\right)^{2}
$$

Now that we know that $\overrightarrow{\vec{M}}$ is SPD we know its inverse exists. We multiply equation (130) by the inverse of $\overrightarrow{\vec{M}}$ to arrive at the following expression.

$$
\overrightarrow{\vec{M}}^{-1} \overrightarrow{\vec{M}} \vec{\delta}=\overrightarrow{\vec{M}}^{-1} 0=\vec{\delta}=0
$$

If $\Delta_{i}=0$ then we know by the definition of $\Delta_{i}$ that all of the scalar fluxes under consideration are equal.

$$
\Delta_{i}=\phi_{i}^{(0)}-\phi_{N_{c}}^{(0)}=0, \quad i=1 \ldots N_{c},
$$

or

$$
\phi_{i}^{(0)}=\phi_{N_{c}}^{(0)}, \quad i=1 \ldots N_{c} .
$$


If we remember our nomenclature for $i$ and $N_{c}$ we can see that equation (7) is indeed the conclusion that we were seeking. The leading-order scalar fluxes are equal for all corners that share a common vertex.

$$
\phi_{c}^{(0)}=\phi_{\tilde{c}_{f e p}}^{(0)} \equiv \phi_{p}^{(0)} \quad \forall c, \tilde{c} \in p .
$$




\section{APPENDIX B \\ REFLECTIVE BOUNDARY CONDITIONS ON ARBITRARY SURFACES}

We have developed a spatial discretization that can solve the transport equation on a grid composed of arbitrary polyhedra. However, we have not described how we handle reflective boundary conditions on arbitrarily oriented surfaces (which may certainly exist in a polyhedral grid!). The algorithm we develop below preserves a net zero current across the reflective boundary. However, the current implementation does not preserve the flux distribution at that surface.

\section{B. 1. Reflection Vectors}

We will develop an algorithm for reflective boundary conditions from the standpoint of code implementation. When solving the transport problem we will sweep over all of the cells in the problem for each angle in our quadrature set. When we first look at one of these quadrature directions we assimilate all of the known information. Usually, this information consists of specified incident angular fluxes or reflected angular fluxes. For the later case the known information is derived from exiting information for that surface. For reflecting surfaces that are orthogonal to the axis planes this is a simple one-to-one relationship. Every exiting direction will reflect into direction that exists in the quadrature set so that $\psi_{s}^{i n c}\left(\vec{\Omega}_{m}\right)=\psi_{s}^{\text {exit }}\left(\vec{\Omega}_{m}^{\prime}\right)$. When reflected off an orthogonal surface the reflected direction can be computed by simply changing the sign on one of the components $\left(\mu_{m}, \eta_{m}, \zeta_{m}\right)$ of $\vec{\Omega}_{m}$.

The reflected direction from an arbitrarily oriented surface is a bit more difficult to calculate. Consider the geometry shown in Figure 31. In this figure, $\vec{A}_{f}$ is the normal area vector of the face shown and $\vec{A}_{f}^{\prime}$ is vector of that has the same direction (perpendicular to the surface) as $\vec{A}_{f}$ but whose length is defined to be the height of the isosceles triangle formed by the vectors $\vec{\Omega}, \vec{\Omega}^{\prime}$ and $2 \vec{r}$.

$$
\vec{A}_{f}^{\prime}=\left(\vec{\Omega} \cdot \frac{\vec{A}_{f}}{\left|\vec{A}_{f}\right|}\right) \frac{\vec{A}_{f}}{\left|\vec{A}_{f}\right|}=\left(\frac{\vec{\Omega} \cdot \vec{A}_{f}}{\vec{A}_{f}^{2}}\right) \vec{A}_{f} .
$$

From this definition and the geometry shown in Figure 31, we can write down a relationship between $\vec{\Omega}$ and $\vec{\Omega}^{\prime}$.

$$
\begin{aligned}
\vec{\Omega}^{\prime} & =\vec{\Omega}-2 \vec{A}_{f}^{\prime}, \\
& =\vec{\Omega}-2 \vec{A}_{f}\left(\frac{\vec{\Omega} \cdot \vec{A}_{f}}{\vec{A}_{f}^{2}}\right)
\end{aligned}
$$


Since we are interested in which directions reflect from a surface into the incoming quadrature direction a more useful relation would be

$$
\vec{\Omega}=\vec{\Omega}^{\prime}+2 \vec{A}_{f}\left(\frac{\vec{\Omega}^{\prime} \cdot \vec{A}_{f}}{\vec{A}_{f}^{2}}\right)
$$

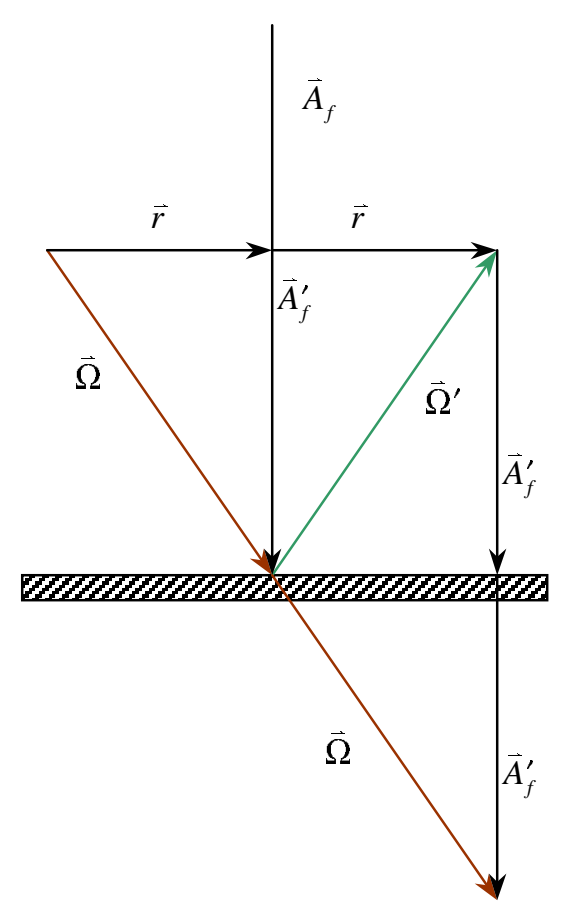

Figure 31. Calculating the reflected direction of a quadrature direction from an arbitrarily oriented surface.

\section{B. 2. Reflection Vectors}

Now that we have relation between the exiting and reflected directions, we must determine how to distribute exiting information (this information exists for directions in our quadrature set) into the reflected angular flux spectrum. This problem is difficult because the exact reflected direction, $\vec{\Omega}^{\prime}$, in general, does not exist in the quadrature set being used for the calculation. So, we must find the exact reflected angular flux, $\psi^{\text {refl }}\left(\vec{\Omega}^{\prime}\right)$, and then redistribute its spectrum into the incident directions that exist in our quadrature set.

The one piece of information that we have for reflective boundaries is that the net current for the surface must be zero. The sum is over all quadrature directions.

$$
\begin{gathered}
\vec{A}_{f} \cdot \vec{J}_{n e t}=0, \\
o r \\
\vec{A}_{f} \cdot \int_{4 \pi} d \Omega \vec{\Omega} \psi(\vec{\Omega})=0 .
\end{gathered}
$$


We can break this equation into exiting and incident (reflected) directions as shown in the next equation.

$$
\vec{A}_{f} \cdot \int_{\vec{A}_{f} \cdot \vec{\Omega}>0} d \Omega \vec{\Omega} \psi^{\text {exit }}(\vec{\Omega})+\vec{A}_{f} \cdot \int_{\vec{A}_{f} \cdot \vec{\Omega}<0} d \Omega \vec{\Omega} \psi^{r e f l}(\vec{\Omega})=0 .
$$

The reflected angular fluxes shown above are exact. If we refer the nomenclature used in Figure 31 we can write the reflected angular flux in terms of the exiting fluxes and their associated reflected directions.

$$
\vec{A}_{f} \cdot \int_{\vec{A}_{f} \cdot \vec{\Omega}^{\prime}>0} d \Omega \vec{\Omega} \psi^{\text {exit }}(\vec{\Omega})+\vec{A}_{f} \cdot \int_{\vec{A}_{f} \cdot \vec{\Omega}^{\prime}<0} d \Omega \vec{\Omega}^{\prime} \psi^{\text {exit }}(\vec{\Omega})=0 .
$$

So far we have only considered the continuous case. We now rewrite equation (150) using the discrete ordinates approximation.

$$
\vec{A}_{f} \cdot \sum_{\vec{A}_{f} \cdot \vec{\Omega}_{j}>0} w_{j} \vec{\Omega}_{j} \psi^{\text {exit }}\left(\vec{\Omega}_{j}\right)+\vec{A}_{f} \cdot \sum_{\vec{A}_{f} \cdot \vec{\Omega}_{j}<0} w_{j} \vec{\Omega}_{j} \psi^{i n c}\left(\vec{\Omega}_{j}\right)=0 .
$$

Since the exact reflection does not exist in the quadrature set we have replaced $\psi^{\text {refl }}$ with $\psi^{\text {inc }}$ to indicate the new distribution of incident angular fluxes.

We can also discretize equation (149) noting that all of the angles of interest already exist in the current quadrature set.

$$
\vec{A}_{f} \cdot \sum_{\vec{A}_{f} \cdot \vec{\Omega}_{j}>0} w_{j} \vec{\Omega}_{j} \psi^{\text {exit }}\left(\vec{\Omega}_{j}\right)+\vec{A}_{f} \cdot \sum_{\vec{A}_{f} \cdot \vec{\Omega}_{j}^{\prime}<0} w_{j} \vec{\Omega}_{j}^{\prime} \underbrace{\psi^{\text {exit }}\left(\vec{\Omega}_{j}\right)}_{-\psi^{\prime \text { ref }}\left(\vec{\Omega}_{j}^{\prime}\right)}=0 .
$$

Now, subtracting equation (152) from equation (151) we arrive at an expression that relates the exact reflection, $\psi^{\text {refl }}\left(\vec{\Omega}_{j}^{\prime}\right)$, to the redistributed reflection, $\psi^{\text {inc }}\left(\vec{\Omega}_{j}\right)$.

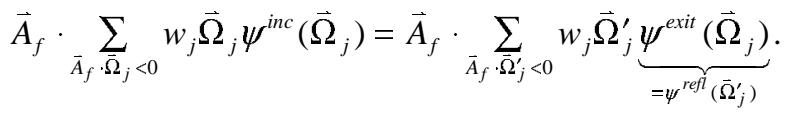

Remember that $\vec{\Omega}_{j}$ are directions that exist in our quadrature set but the directions represented by $\vec{\Omega}_{j}{ }^{\prime}$ are not in our quadrature set. Also note that $\psi^{\text {exit }}\left(\vec{\Omega}_{j}\right) \neq \psi^{i n c}\left(\vec{\Omega}_{j}\right)$ since the $\psi^{\text {exit }}$ are all exiting directions and $\psi^{i n c}$ are all incident directions. The full set of quadrature directions is the sum of these two sets.

Now, examine only one incoming direction that exists in our quadrature set $\left(\vec{A}_{f} \cdot \vec{\Omega}_{m}<0\right)$ and all of the exact reflected directions that are not in our quadrature set $\left(\vec{A}_{f} \cdot \vec{\Omega}_{m}^{\prime}<0\right)$. We will assume that each of the exact reflections contribute a fraction of their angular flux into the quadrature set direction of interest. The portion of their exiting angular flux that contributes to the direction of interest is denoted by a weighting parameter, $\alpha_{j}^{m}$, so that

$$
w_{m}\left(\vec{A}_{f} \cdot \vec{\Omega}_{m}\right) \psi^{i n c}\left(\vec{\Omega}_{m}\right)=\vec{A}_{f} \cdot \sum_{\vec{A}_{f} \cdot \vec{\Omega}_{j}^{\prime}<0} w_{j} \vec{\Omega}_{j}^{\prime} \alpha_{j}^{m} \psi^{i n c}\left(\vec{\Omega}_{j}^{\prime}\right) .
$$


The weighting function $\alpha_{j}^{m}$ must be selected so that after all reflected angular fluxes are calculated the net current across the face remains zero. Note that this parameter will depend on some relationship between the angle of interest, $\vec{\Omega}_{m}$, and all of the exact reflected directions, $\vec{\Omega}_{j}^{\prime}$. To see if we preserve zero net current we can sum equation (154) over all incoming quadrature directions.

$$
\begin{aligned}
& \sum_{\vec{A}_{f} \cdot \vec{\Omega}_{m}<0}\left[w_{m}\left(\vec{A}_{f} \cdot \vec{\Omega}_{m}\right) \psi^{i n c}\left(\overrightarrow{\boldsymbol{\Omega}}_{m}\right)\right]=\sum_{\vec{A}_{f} \cdot \vec{\Omega}_{m}<0}\left[\vec{A}_{f} \cdot \sum_{\vec{A}_{f} \cdot \vec{\Omega}_{j}^{\prime}<0} w_{j} \overrightarrow{\boldsymbol{\Omega}}_{j}^{\prime} \alpha_{j}^{m} \psi^{\text {exit }}\left(\overrightarrow{\boldsymbol{\Omega}}_{j}^{\prime}\right)\right], \\
& \vec{A}_{f} \cdot \sum_{\vec{A}_{f} \cdot \vec{\Omega}_{m}<0} w_{m} \vec{\Omega}_{m} \psi^{i n c}\left(\vec{\Omega}_{m}\right)=\vec{A}_{f} \cdot \sum_{\vec{A}_{f} \cdot \vec{\Omega}_{j}^{\prime}<0} w_{j} \vec{\Omega}_{j}^{\prime} \psi^{e x i t}\left(\vec{\Omega}_{j}^{\prime}\right) \sum_{\vec{A}_{f} \cdot \vec{\Omega}_{m}<0} \alpha_{j}^{m} .
\end{aligned}
$$

Comparing this with equation (153) we see that to preserve zero net current $\alpha_{j}^{m}$ must have the following property.

$$
\sum_{\vec{A}_{f} \cdot \hat{\Omega}_{m}<0} \alpha_{j}^{m}=1 \quad \forall j:: \vec{A}_{f} \cdot \vec{\Omega}_{j}^{\prime}<0 .
$$

This requirement leaves us with a great deal of freedom when choosing a form for $\alpha_{j}^{m}$. Since it makes physical sense to make exact reflections, $\vec{\Omega}_{j}^{\prime}$, that are near the direction of interest to have more effect on the angular flux of interest, $\psi^{i n c}\left(\vec{\Omega}_{m}\right)$, we will use a $1 / \sin \left(\theta_{j}^{m}\right)$ weight function. We have chosen $\theta_{j}^{m}$ so that it is half the angle between $\vec{\Omega}_{m}$ and $\vec{\Omega}_{j}^{\prime}$. This geometry is shown in Figure 32 .

$$
\alpha_{j}^{m}=\frac{1 / \sin \left(\theta_{j}^{n}\right)}{\sum_{\vec{A}_{f} \cdot \vec{\Omega}_{m}<0} 1 / \sin \left(\theta_{j}^{n}\right)}, \quad \theta_{j}^{m}=\frac{1}{2} \cos ^{-1}\left(\vec{\Omega}_{m} \cdot \vec{\Omega}_{j}^{\prime}\right) .
$$

We note that this definition of $\alpha_{j}^{n}$ satisfies the requirement of equation (156).

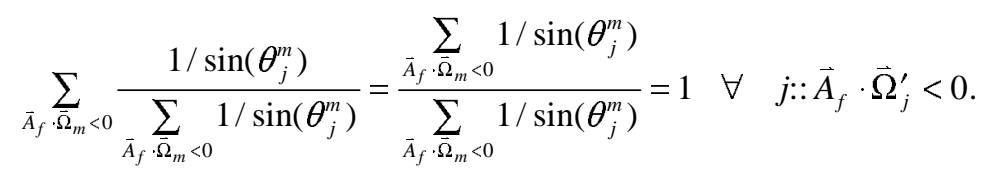

We define $\theta_{j}^{m}$ to be half the angle between $\vec{\Omega}_{m}$ and $\vec{\Omega}_{j}^{\prime}$ so those vectors with $\pi$ radians separation have the minimum importance and vectors with 0 radians separation have the maximum importance.

We can now rewrite equation (154) using a component form. In this formulation $\psi_{j}^{m}$ represents the fraction of the exactly reflected angular flux, $\psi^{i n c}\left(\vec{\Omega}_{j}^{\prime}\right)$, which contributes to the incoming angular flux, $\psi^{i n c}\left(\vec{\Omega}_{m}\right)$, that is in our quadrature set.

$$
\begin{aligned}
\psi^{i n c}\left(\vec{\Omega}_{m}\right) & =\sum_{\vec{A}_{f} \cdot \vec{\Omega}_{j}^{\prime}<0} \frac{w_{j}}{w_{m}} \frac{\left(\vec{A}_{f} \cdot \vec{\Omega}_{j}^{\prime}\right)}{\left(\vec{A}_{f} \cdot \vec{\Omega}_{m}\right)} \alpha_{j}^{m} \psi^{i n c}\left(\vec{\Omega}_{j}^{\prime}\right), \\
& =\sum_{\vec{A}_{f} \cdot \vec{\Omega}_{j}^{\prime}<0} \beta_{j}^{m} \psi_{j}^{m},
\end{aligned}
$$


where

$$
\beta_{j}^{m} \equiv \frac{w_{j}}{w_{m}} \frac{\left(\vec{A}_{f} \cdot \vec{\Omega}_{j}^{\prime}\right)}{\left(\vec{A}_{f} \cdot \vec{\Omega}_{m}\right)} \quad \text { and } \quad \psi_{j}^{m} \equiv \alpha_{j}^{m} \psi^{i n c}\left(\vec{\Omega}_{j}^{\prime}\right)
$$

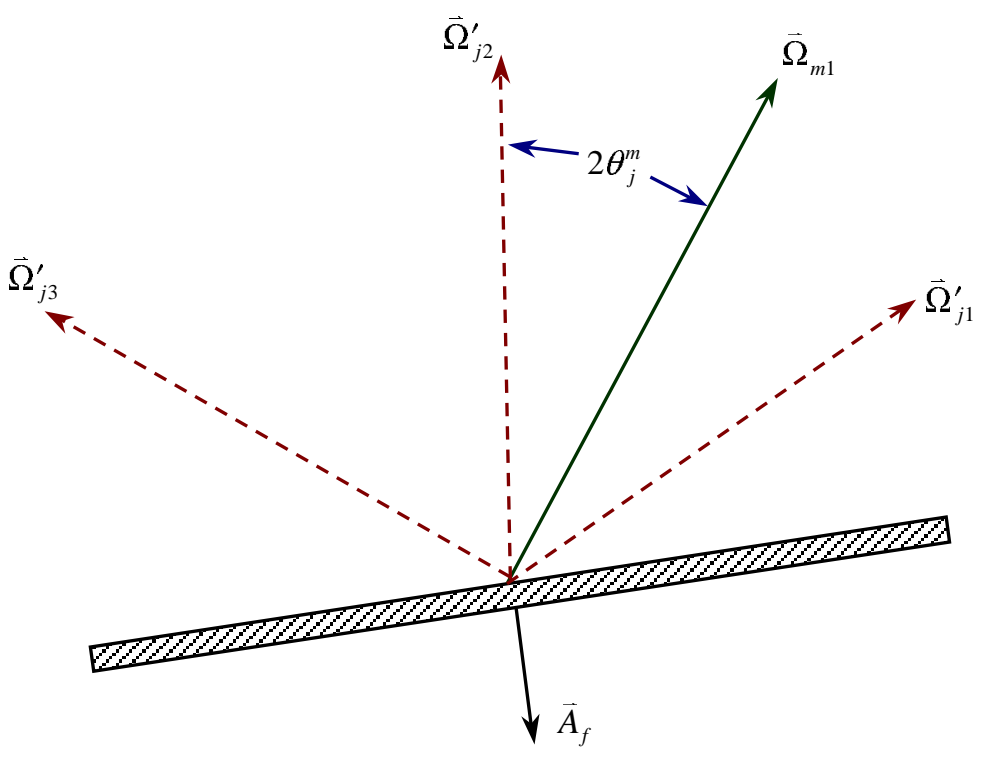

Figure 32. Angle of interest and its relation to exact reflected angles that are not in the quadrature set.

In practice, we use equation (159) to calculating both $\beta_{j}^{m}$ and $\psi_{j}^{m}$ for the incoming direction of interest. Then we simply sum their product over all exiting directions since $\psi^{i n c}\left(\vec{\Omega}_{j}^{\prime}\right)=\psi^{\text {exit }}\left(\vec{\Omega}_{j}\right)$ for all $j$ so that $\vec{A}_{f} \cdot \vec{\Omega}_{j}>0$.

This implementation preserves the zero net current across the reflective surface. However, it does not preserve the exiting angular flux distribution upon reflection. In numerical tests this algorithm has performed reasonably well which the exception of reflective boundaries on the surface of spheres or on the surface of cylinders. In these particular cases, the boundary flux is artificially elevated. This is a known problem in reactor physics and can be remedied by using a white boundary condition instead of a truly reflective boundary condition when it is known that the reflected flux should be isotropic. 


\section{B. 3. White boundary condition}

The white boundary condition preserves a zero net current across a surface but enforces the reflected angular flux to be isotropic regardless of the angular distribution of the exiting flux. The reflected white angular flux is represented as follows:

$$
\begin{aligned}
& -\vec{A}_{f} \cdot \sum_{\vec{A}_{f} \cdot \vec{\Omega}_{j}<0} w_{j} \vec{\Omega}_{j} \psi^{i n c}\left(\vec{\Omega}_{j}\right)=\vec{A}_{f} \cdot \sum_{\vec{A}_{f} \cdot \bar{\Omega}_{j}>0} w_{j} \vec{\Omega}_{j} \psi^{\text {exit }}\left(\vec{\Omega}_{j}\right), \\
& \sum_{\vec{A}_{f} \cdot \bar{\Omega}_{j}<0} w_{j}\left|\vec{A}_{f} \cdot \vec{\Omega}_{j}\right| \psi_{i s o}^{i n c}\left(\vec{\Omega}_{j}\right)=\vec{A}_{f} \cdot \sum_{\vec{A}_{f} \cdot \sum_{j}>0} w_{j} \vec{\Omega}_{j} \psi^{\text {exit }}\left(\vec{\Omega}_{j}\right), \\
& \text { s.t. } \quad \psi_{i s o}^{i n c}=\frac{\sum_{\bar{A}_{f} \cdot \vec{\Omega}_{j}>0} w_{j} \vec{A}_{f} \cdot \vec{\Omega}_{j} \psi^{\text {exit }}\left(\vec{\Omega}_{j}\right)}{\sum_{\vec{A}_{f} \cdot \vec{\Omega}_{j}<0} w_{j}\left|\vec{A}_{f} \cdot \vec{\Omega}_{j}\right|} .
\end{aligned}
$$

Where $\psi_{\text {iso }}^{\text {inc }}$ has the same value for all incoming directions. 


\section{APPENDIX C}

\section{CORNER BASED 3D UCBL}

In an attempt to create a polyhedral scheme that is conservative on corners, second-order accurate and is less taxing computationally we outline the requirements for a corner based UCBL scheme. We do not intend on doing a full analysis of this scheme but we do want to present a possible framework.

\section{1. Geometry and Nomenclature}

In the wedge based scheme that is considered in the bulk of this work we consider polyhedral corners that are subdivided into tetrahedral wedges as shown in Figure 1. From this figure we notice that the following vertices bound a corner:

(z) The cell midpoint

(p) A singe vertex of the zone

(e) Edge midpoints of zone $z$ touching $p$

(f) Face midpoints of zone $z$ touching $p$

We note that a surface that separates two corners in a single cell is defined by and edge midpoint and a surface that separates two corners that reside in different cells is defined by a face midpoint. It should also be pointed out that the interfacing surface between any two corners is not necessarily a planar surface; In general it is the union of two planar triangular surfaces. An external (cell-to-cell) interface is the union of two $f e p$ wedge facelets with the same $f$ and $p$ but different $e$ points. An internal (within-cell) interface is the union of two $f e z$ wedge facelets with the same $e$ and $z$ but different $f$ points.

For now, we will assume that the areas of the corner interfaces are simple combinations of the wedge facelet area vectors.

$$
\begin{aligned}
& \vec{A}_{e z}=\vec{A}_{f_{1} e z}+\vec{A}_{f_{2} e z}, \\
& \vec{A}_{f p}=\vec{A}_{f e_{1} p}+\vec{A}_{f e_{2} p} .
\end{aligned}
$$

We assume that all area vectors are pointed outward from the corner volume. In equation (162) we also assume that $f_{1}$ and $f_{2}$ share the same face with the vector $e z$. Similarly we assume that $e_{1}$ and $e_{2}$ share the same face as the vector $f p$. 


\section{2. Balance}

We already have a balance equation for a corner-based scheme (see equation (6)). We rewrite this equation using our new nomenclature for consistency in this Appendix.

$$
\sum_{f \in c o r n e r} \vec{A}_{f p} \cdot \vec{\Omega}_{m} \psi_{m, f p}+\sum_{e \in c o r n e r} \vec{A}_{e z} \cdot \vec{\Omega}_{m} \psi_{m, e z}+V_{c} \sigma_{t, c} \psi_{m, c}=V_{c} Q_{m, c} \cdot
$$

Remember that half of the corner surfaces are defined by face midpoints and the other half are defined my edge midpoints.

\section{3. Closure Equations}

Instead of four closure equations, our corner-based scheme will only have two, one for external surfaces and one for internal surfaces. We will use the same step closure that is used in other CB discretizations.

$$
\psi_{m, f p}= \begin{cases}\psi_{m, c} & \vec{A}_{f p} \cdot \vec{\Omega}_{m}>0, \\ \psi_{m, \tilde{c}_{f p}} & \vec{A}_{f p} \cdot \vec{\Omega}_{m}<0, \quad \vec{r} \notin \Gamma, \\ \psi_{m, \tilde{c}_{f p}}^{i n c} & \vec{A}_{f p} \cdot \vec{\Omega}_{m}<0, \quad \vec{r} \in \Gamma .\end{cases}
$$

We have used the notation $\tilde{c}_{f p}$ to represent the shared $f p$ face of the adjacent corner and $\Gamma$ to represent the boundary of the problem. It may be advantageous in the future to add higher order terms to this closure to improve performance in thin problems. However, we need this step closure to hold true to leading order in an asymptotic analysis if we are to capture a consistent diffusion discretization.

The internal surface closure is very similar to $f e z$ closure in our wedge-based scheme. It is the sum of the corner average angular flux, a difference between sources and an extrapolation across the cell. The extrapolation term needs to be designed to capture second-order accuracy in thin problems.

$$
\psi_{m, e z}= \begin{cases}\psi_{m, c}+\frac{1}{2}\left(\left.\frac{Q_{m}}{\sigma_{t}}\right|_{\tilde{c}_{e z}}-\left.\frac{Q_{m}}{\sigma_{t}}\right|_{c}\right)+\gamma\left(\tau_{m, c}\right)\left(\left|\vec{A}_{o p} \cdot \vec{\Omega}_{m}\right|-\vec{A}_{o p} \cdot \vec{\Omega}_{m}\right)\left[\psi_{m, c}-\psi_{m, c}^{o p}\right], & \vec{A}_{f p} \cdot \vec{\Omega}_{m}>0, \\ \psi_{m, c}+\frac{1}{2}\left(\left.\frac{Q_{m}}{\sigma_{t}}\right|_{c}-\left.\frac{Q_{m}}{\sigma_{t}}\right|_{\tilde{\tau}_{e z}}\right)+\gamma\left(\tau_{m, \tilde{c}_{e z}}\right)\left(\left|\vec{A}_{o p} \cdot \vec{\Omega}_{m}\right|-\vec{A}_{o p} \cdot \vec{\Omega}_{m}\right)\left[\psi_{m, \tilde{c}_{e z}}-\psi_{m, \tilde{c}_{e z}}^{o p}\right], & \vec{A}_{f p} \cdot \vec{\Omega}_{m}<0 .\end{cases}
$$

The terminology $o p$ is used to signify the opposite face. In general there will be no opposite face for 3D problems. However, there will always be at least one (and often more) face that can be considered to be opposite. We will consider an opposite face to mean any corner face that does not share the same edge point as the $e z$ face under consideration.

We will define $\psi_{m, c}^{o p}$ to be an area-weighted average over incoming opposite faces.

$$
\left(\left|\vec{A}_{o p} \cdot \vec{\Omega}_{m}\right|-\vec{A}_{o p} \cdot \vec{\Omega}_{m}\right) \psi_{m, c}^{o p} \equiv \sum_{f p \in o p}\left(\left|\vec{A}_{f p} \cdot \vec{\Omega}_{m}\right|-\vec{A}_{f p} \cdot \vec{\Omega}_{m}\right) \psi_{m, f p} .
$$

Where we define the opposite area vector so that

$$
\vec{A}_{o p} \cdot \vec{\Omega}_{m} \equiv \sum_{f p \in o p} \vec{A}_{f p} \cdot \vec{\Omega}_{m} .
$$


The nomenclature $f p \in o p$ represents all of the $f p$ faces in that are opposite of $e z$. Using these two definitions of opposite in the $e z$ closure have the following expression.

$$
\psi_{m, e z}= \begin{cases}\psi_{m, c}+\frac{1}{2}\left(\left.\frac{Q_{m}}{\sigma_{t}}\right|_{\tilde{c}_{e z}}-\left.\frac{Q_{m}}{\sigma_{t}}\right|_{c}\right)+\gamma\left(\tau_{m, c}\right) \sum_{f p \in o p}\left(\left|\vec{A}_{f p} \cdot \vec{\Omega}_{m}\right|-\vec{A}_{f p} \cdot \vec{\Omega}_{m}\right)\left(\psi_{m, c}-\psi_{m, f p}\right), & \vec{A}_{f p} \cdot \vec{\Omega}_{m}>0, \\ \psi_{m, c}+\frac{1}{2}\left(\left.\frac{Q_{m}}{\sigma_{t}}\right|_{c}-\left.\frac{Q_{m}}{\sigma_{t}}\right|_{\tilde{c}_{e z}}\right)+\gamma\left(\tau_{m, \tilde{c}_{e z}}\right) \sum_{f p \in o p}\left(\left|\vec{A}_{f p} \cdot \vec{\Omega}_{m}\right|-\vec{A}_{f p} \cdot \vec{\Omega}_{m}\right)\left(\psi_{m, \tilde{c}_{e z}}-\psi_{m, f p}\right), & \vec{A}_{f p} \cdot \vec{\Omega}_{m}<0 .\end{cases}
$$

We are currently using the same functional for $\gamma\left(\tau_{m, c}\right)$ as UCB uses in 2D.

$$
\begin{aligned}
& \gamma\left(\tau_{m, c}\right) \equiv \frac{1}{\tau_{e z}} \frac{3+4 \tau_{e z}+4 \alpha_{\infty} \tau_{e z}^{2}}{2+2 \tau_{e z}+4 \tau_{e z}^{2}}, \\
& \text { with } \tau_{e z}=\frac{\sigma_{t, c} V_{c}}{\left|\vec{A}_{e z} \cdot \vec{\Omega}_{m}\right|} .
\end{aligned}
$$

Equations (163), (164), (168) and (169) define our new scheme. Doing away with wedge elements should reduce the total memory requirements for our algorithm as well as reducing the time it takes to complete a single sweep of the mesh. Additionally, the use of $\gamma\left(\tau_{m, c}\right)$ should allow our algorithm to be second-order accurate. We were able to maintain the leading order terms in all of these closures. This means that our corner-based algorithm should capture the same leading order solution as the wedge-based scheme. 


\section{APPENDIX D}

\section{TRUNCATION ERROR FOR 2D UCBL}

In the body of this work tests were run that indicate that UCBL is a first-order accurate scheme. We have also stated that Godunov's Theorem applies to UCBL. While both of these analyses indicate that UCBL is a first-order accurate scheme, we will perform one additional analysis to support this claim. In this analysis we only consider the on the 2D UCBL equations and we will closely follow the work of Mathews for this analysis. ${ }^{14}$

\section{1. The $2 D$ UCBL Equations}

In 2D corner balance methods break polygonal cells into quadrilateral corners. 2D UCBL will divide each of these corners into two triangular wedges. For this truncation error analysis we will only consider rectangular cells that are divided into eight wedges each as shown in Figure 33. The figure shows the labeled vertices that represent $(p)$ oint, $(e)$ dge midpoint and $(z)$ one center values. For discrete ordinate transport there are two types of streaming across this cell. For $\alpha<1$ particles incident on the bottom face will cross the cell diagonal and exit out the top face. If $\alpha>1$ then particles entering from the bottom face will only exit out the right face. The actual definition of $\alpha$ is based on geometric factors and the quadrature direction of interest. This definition is presented and discussed below.

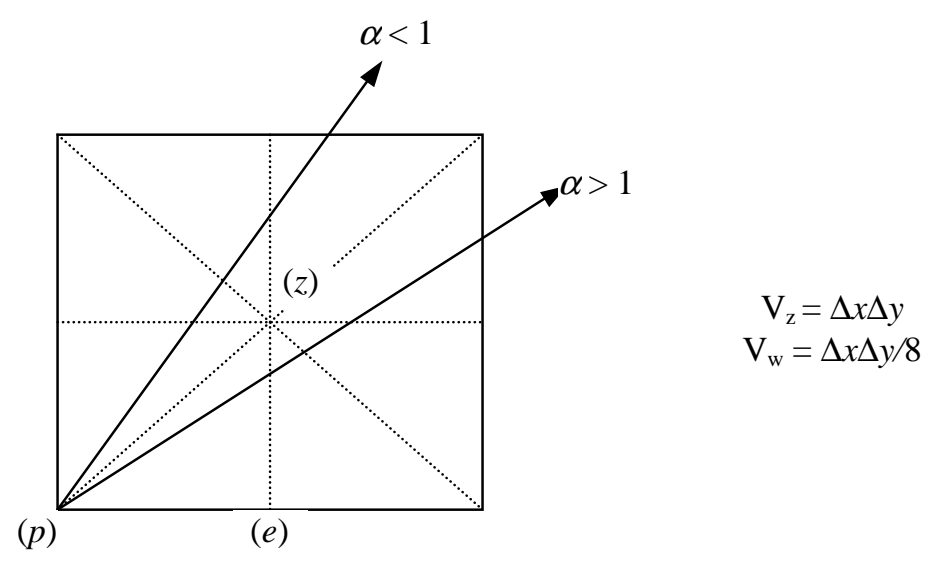

Figure 33. A rectangular wedge divided into eight wedges.

We can integrate the transport equation (see equation (4)) over the volume a wedge.

$$
\vec{\Omega} \cdot\left(\vec{A}_{e z} \psi_{e z}+\vec{A}_{p e} \psi_{p e}+\vec{A}_{p z} \psi_{p z}\right)+\sigma_{t} V_{w} \psi_{w}=0 .
$$


For this particular case we assume there is no scattering, fission nor any distributed sources. The two-letter subscripts refer to edge-averaged quantities and the $w$ subscript refers to area-averaged quantities for the current wedge. The 3D closure equations simplify in a source-free purely absorbing 2D implementation.

$$
\begin{aligned}
& \psi_{e z}=\psi_{w}+\gamma\left(\psi_{w}-\psi^{i n c}\right), \quad \vec{A}_{e z} \cdot \vec{\Omega}>0, \\
& \psi_{p z}=\psi_{w}+\beta\left(\psi_{w}-\psi^{i n c}\right), \quad \vec{A}_{p z} \cdot \vec{\Omega}>0, \\
& \psi_{p e}= \begin{cases}\psi_{w}, & \vec{\Omega} \cdot \vec{A}_{p e}>0, \\
\psi_{p e}^{i n c}, & \vec{\Omega} \cdot \vec{A}_{p e}<0 .\end{cases}
\end{aligned}
$$

These closures use parameters similar to those introduced in our3D UCBL development. These parameters are defined here.

$$
\gamma \equiv \frac{3+4 \tau_{e z}}{2 \tau_{e z}\left(1+\tau_{e z}+2 \tau_{e z}^{2}\right)}, \quad \beta \equiv\left(1+\frac{1}{3} \tau_{p z}+\frac{1}{18} \tau_{p z}^{2}\right)^{-1}, \quad \tau_{x x} \equiv \frac{\sigma_{t} V_{w}}{\vec{\Omega} \cdot \vec{A}_{x x}} \quad \text { and } \quad \psi^{i n c} \equiv \frac{\sum_{\vec{\Omega} \cdot \vec{A}_{i}<0} \vec{\Omega} \cdot \vec{A}_{i} \psi_{i}}{\sum_{\vec{\Omega} \cdot \vec{A}_{i}<0} \vec{\Omega}_{i} \cdot \vec{A}_{i}}
$$

The optical thickness definition is valid for three distinct cases $(x x=e z, p z$ and $p e)$.

For this exercise we will only consider positive quadrature directions.

$$
\vec{\Omega}=\mu \hat{e}_{x}+\eta \hat{e}_{y}, \mu>0 \text { and } \eta>0 .
$$

The balance equation for a corner can be obtained by integrating the transport equation over the area of a corner. Using Green's Theorem and the definition of $\vec{\Omega}$ from above we arrive at the following equation.

$$
\frac{\eta \Delta x}{2} \psi_{T}+\frac{\mu \Delta y}{2} \psi_{R}-\frac{\eta \lambda x}{2} \psi_{B}-\frac{\mu \Lambda y}{2} \psi_{L}+\sigma_{t} V_{c} \psi_{c}=0
$$

We have used the subscript $c$ to indicate corner-area-averaged quantities. The subscripts $T, B, L$ and $R$ indicate surface averaged quantities for the top, bottom, left and right faces respectively. This equation can be further simplified by defining to additional parameters.

$$
\alpha \equiv \frac{\mu \Delta y}{\eta \Delta x} \text { and } \quad \varepsilon_{y} \equiv \frac{\sigma_{t} \Delta y}{\eta} .
$$

Our corner-based balance equation now has the following form.

$$
\psi_{T}+\alpha \psi_{R}-\psi_{B}-\alpha \psi_{L}+\frac{1}{2} \varepsilon_{y} \psi_{c}=0
$$

\section{2. Attenuation Across a Row of Cells}

If we consider a row of cells as shown in Figure 34 with a beam incident on the bottom face of cell 0 we notice that 2D UCBL will propagate this beam through the entire row to the right if $\alpha>1$. Following the work of Mathews we can find the exiting flux for the top face of this row of cells. The attenuation of our incident beam will be the ratio of the exiting flux to the incident flux.

$$
a_{\text {discrete }}=\frac{\sum_{i=0}^{N} \psi_{T, i}}{\psi_{B, 0}}
$$


The analytic attenuation is

$$
a_{\text {exact }}=2 e^{-\varepsilon_{y}} \text {. }
$$

The exact solution has a coefficient of 2 because we assume that there is an incident flux of equal magnitude on both bottom corner faces of cell 0 .

By comparing the Taylor series expansion of these two equations about $\varepsilon_{y}=0$ we can determine the truncation error of 2D UCBL. For this analysis to be accurate we must examine the discrete attenuation in the limit where $N \rightarrow \infty$.

Exiting flux for entire top face.

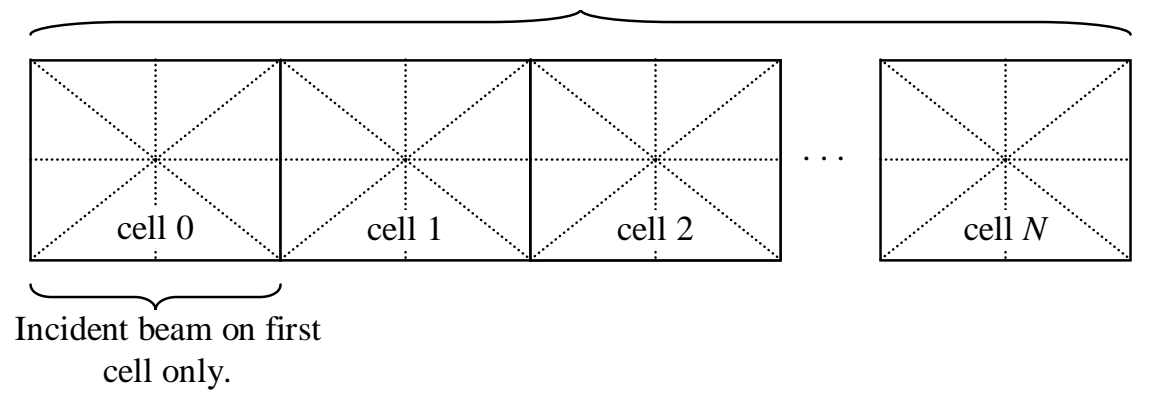

Figure 34. A row of rectangular cells with an incident beam in cell zero.

To determine the total attenuation we must relate the outgoing fluxes on all of the top surfaces to the incident flux from the bottom surface of the first cell. For cell 0 we have the following relation

$$
\begin{aligned}
\psi_{T, 0} & =\left(g_{B 1 T 4}+g_{B 1 T 3_{(4)}}+g_{B 1 T 3_{(2)}}\right) \psi_{B 1,0}+\left(g_{B 2 T 3}\right) \psi_{B 2,0} \\
& +\left(g_{L 1 T 4}+g_{L 1 T 3(4)}+g_{L 1 T 3_{(2)}}\right) \psi_{L 1,0}+\left(g_{L 4 T 4}+g_{L 4 T 3}\right) \psi_{L 4,0} .
\end{aligned}
$$

In this equation the $g$-factors relate incident information to exiting information. For example $g_{B I T 4}$ is the fraction of $\psi_{B 1,0}$ that contributes to $\psi_{T, 0}\left(=\psi_{T 4,0}+\psi_{T 3,0}\right)$. We have labeled the corner surfaces as shown in Figure 35. If we only consider a one cell system $(N=1$ in Figure 34 and equation (177)) then the attenuation would be

$$
a_{0}=g_{B 1 T 4}+g_{B 1 T 3_{(4)}}+g_{B 1 T 3_{(2)}}+g_{B 2 T 3},
$$

since the incident flux on the left is zero and the two incident values on the bottom are equal. 
By developing expressions for the attenuation of a 2-cell problem, a 3-cell problem and a few more we can show that the attenuation for an $N$-cell $(\mathrm{N}>1)$ problem is a recursive relation,

$$
\begin{aligned}
a_{N}= & a_{N-1}+\left[\left(g_{L 1 T 4}+g_{L 1 T 3(4)}+g_{L 1 T 3(2)}\right)\left(g_{B 1 R 2}+g_{B 2 R 2}\right)\right]\left(g_{L 1 R 2}\right)^{i} \\
& +\left[\left(g_{L 4 T 4}+g_{L 4 T 3}\right)\left(g_{B 1 R 2}+g_{B 2 R 2}\right)\right]\left(g_{L 1 R 3_{(2)}}+g_{L 1 R 3_{(4)}}\right) \sum_{k=0}^{i-1}\left(g_{L 1 R 2}\right)^{i-1-k}\left(g_{L 4 R 3}\right)^{k} \\
& +\left[\left(g_{L 4 T 4}+g_{L 4 T 3}\right)\left(g_{B 1 R 3_{(2)}}+g_{B 1 R 3_{(4)}}+g_{B 2 R 3}\right)\right]\left(g_{L 4 R 3}\right)^{i} .
\end{aligned}
$$

Where $a_{0}$ is defined by equation (180).

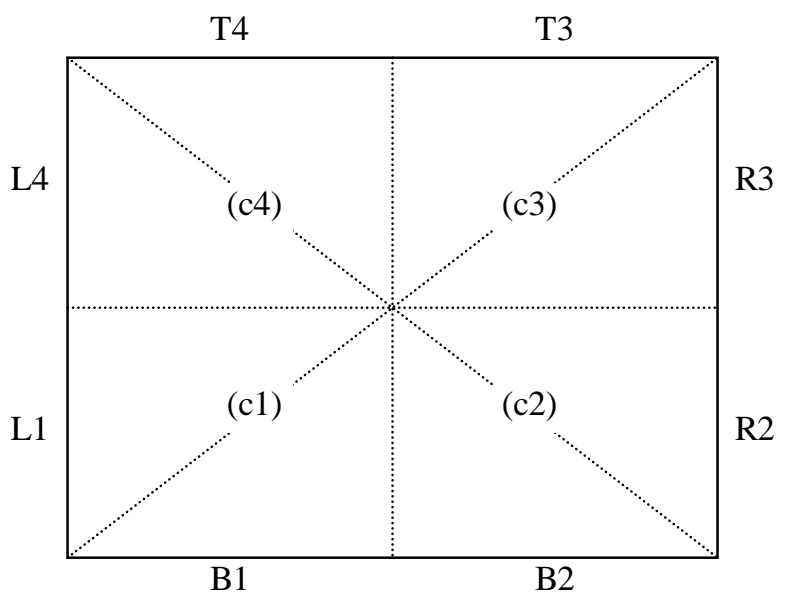

Figure 35. Nomenclature for corner surfaces.

We can remove the recursive nature of this relation by implementing summations.

$$
\begin{aligned}
a_{N}= & {\left[g_{B 1 T 4}+g_{B 1 T 3_{(4)}}+g_{B 1 T 3_{(2)}}+g_{B 2 T 3}\right] } \\
& +\left[\left(g_{L 1 T 4}+g_{L 1 T 3_{(4)}}+g_{L 1 T 3_{(2)}}\right)\left(g_{B 1 R 2}+g_{B 2 R 2}\right)\right] \sum_{i=0}^{N-1}\left(g_{L 1 R 2}\right)^{i} \\
& +\left[\left(g_{L 4 T 4}+g_{L 4 T 3}\right)\left(g_{B 1 R 2}+g_{B 2 R 2}\right)\right]\left(g_{L 1 R 3_{(2)}}+g_{\left.L 1 R 3_{(4)}\right)}\right) \sum_{i=1}^{N-1 i-1} \sum_{k=0}\left(g_{L 1 R 2}\right)^{i-1-k}\left(g_{L 4 R 3}\right)^{k} \\
& +\left[\left(g_{L 4 T 4}+g_{L 4 T 3}\right)\left(g_{B 1 R 3_{(2)}}+g_{B 1 R 3_{(4)}}+g_{B 2 R 3}\right)\right] \sum_{i=0}^{N-1}\left(g_{L 4 R 3}\right)^{i} .
\end{aligned}
$$

If $N \rightarrow \infty$ then we can use the following mathematical property for sums of powers.

$$
\sum_{i=0}^{\infty} \rho^{i}=\frac{1}{1-\rho}
$$

This relation holds for all $\rho<1$. Our $g$-factors have both attenuation and beam spread so they meet this requirement. Thus for an infinite row of cells the attenuation will be. 


$$
\begin{aligned}
a_{N}= & {\left[g_{B 1 T 4}+g_{B 1 T 3_{(4)}}+g_{B 1 T 3_{(2)}}+g_{B 2 T 3}\right] } \\
& +\frac{\left(g_{L 1 T 4}+g_{L 1 T 3_{(4)}}+g_{L 1 T 3_{(2)}}\right)\left(g_{B 1 R 2}+g_{B 2 R 2}\right)}{1-g_{L 1 R 2}} \\
& +\frac{\left(g_{L 4 T 4}+g_{L 4 T 3}\right)\left(g_{B 1 R 3_{(2)}}+g_{B 1 R 3_{(4)}}+g_{B 2 R 3}\right)}{1-g_{L 4 R 3}} \\
& +\frac{\left(g_{L 4 T 4}+g_{L 4 T 3}\right)\left(g_{B 1 R 2}+g_{B 2 R 2}\right)\left(g_{L 1 R 3_{(2)}}+g_{\left.L 1 R 3_{(4)}\right)}\right)}{\left(1-g_{L 1 R 2}\right)\left(1-g_{L 4 R 3}\right)}
\end{aligned}
$$

The $g$-factors will be functions of $\alpha$ and $\varepsilon_{y}$ and so our Taylor series expansion for the discrete attenuation will also be a function of these two variables.

\section{3. Determination of the G-factors.}

The $g$-factors relate an outgoing flux to an incoming flux for a single cell. We can use the 2D UCBL balance and closure equations to determine these $g$-factors. Remember that we are only considering positive directions $(\mu>0$ and $\eta>0)$. We number the 8 wedges in our cell in a counter clockwise fashion. Wedge 1 is in the top-left half of corner 1 (see Figure 36).

If we consider wedge 1 we note that the $2 \mathrm{D}$ UCBL balance equation has the following form:

$$
\vec{\Omega} \cdot \vec{A}_{e z} \psi_{e z}+\vec{\Omega} \cdot \vec{A}_{p e} \psi_{p e}+\vec{\Omega} \cdot \vec{A}_{p z} \psi_{p z}+\frac{\Delta x \eta}{8} \varepsilon_{y} \psi_{w}=0 .
$$

For the case that we are studying $\alpha$ is greater than one. This means that the pe-surface will be the only incident surface for wedge 1 .

$$
\psi_{w 1}^{i n c}=\psi_{p e, w 1}^{i n c} .
$$

For our specific case we can evaluate the omega dot area terms.

$$
\vec{\Omega} \cdot \vec{A}_{e z}=\frac{1}{2} \Delta x \eta, \quad \vec{\Omega} \cdot \vec{A}_{p e}=-\frac{1}{2} \Delta y \mu, \quad \vec{\Omega} \cdot \vec{A}_{p z}=-\frac{1}{2}(\Delta x \eta-\Delta y \mu) .
$$

Using these dot product values in equation (185) produces the following balance equation.

$$
\frac{1}{2} \Delta x \eta \psi_{e z, w 1}-\frac{1}{2}(\Delta x \eta-\Delta y \mu) \psi_{p z, w 1}+\frac{\Delta x \eta}{8} \varepsilon_{y} \psi_{w 1}=\frac{1}{2} \Delta y \mu \psi_{p e, w 1}^{i n c} .
$$

If we divide through by $\frac{1}{2} \Delta x \eta$ and use the parameter definitions for $\alpha$ and $\varepsilon_{y}$, the wedge 1 balance equation reduces to

$$
\psi_{e z, w 1}+(\alpha-1) \psi_{p z, w 1}+\frac{1}{4} \varepsilon_{y} \psi_{w 1}=\alpha \psi_{p e, w 1}^{i n c} .
$$

The exiting surface intensities are known from the closure equations.

$$
\psi_{e z, w 1}=\psi_{w 1}\left(1+\gamma_{w 1}\right)-\gamma_{w 1} \psi_{p e, w 1}^{i n c} \quad \text { and } \quad \psi_{p z, w 1}=\psi_{w 1}\left(1+\beta_{w 1}\right)-\beta_{w 1} \psi_{p e, w 1}^{i n c} .
$$

Using these closures in equation (188) we can solve for the area-averaged angular flux for wedge 1.

$$
\psi_{w 1}=\frac{\alpha+\gamma_{w 1}-(1-\alpha) \beta_{w 1}}{1+\gamma_{w 1}-(1-\alpha)\left(1+\beta_{w 1}\right)+\frac{1}{4} \varepsilon_{y}} \psi_{p e, w 1}^{i n c} .
$$




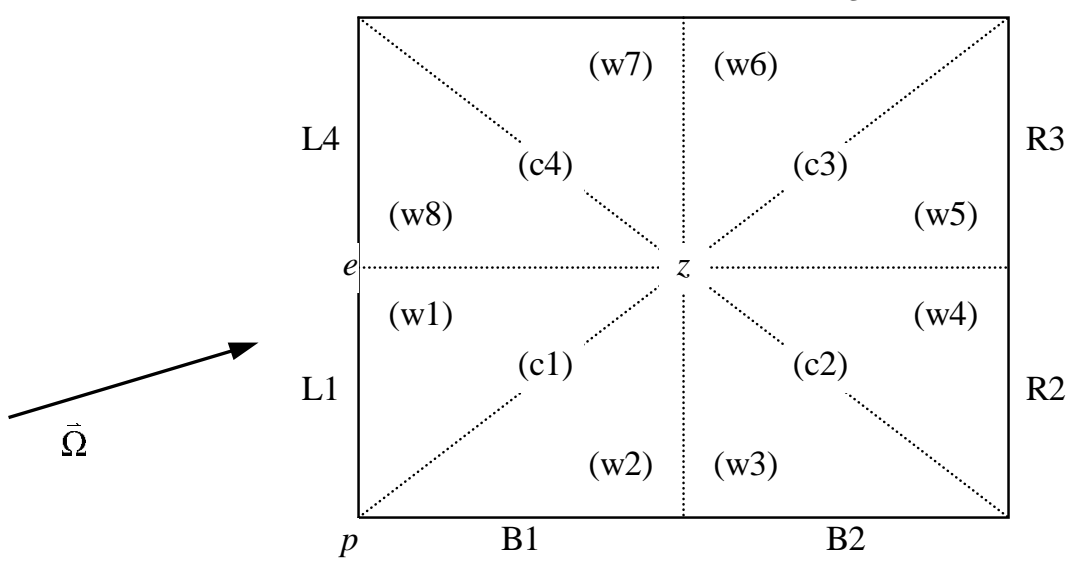

Figure 36. Numbering scheme for 2D wedges.

This, in turn, allows us to solve for the surface-averaged exiting angular fluxes for wedge 1.

$$
\begin{aligned}
& \psi_{e z, w 1}=\left[\frac{\left[\alpha+\gamma_{w 1}-(1-\alpha) \beta_{w 1}\right]\left(1+\gamma_{w 1}\right)}{1+\gamma_{w 1}-(1-\alpha)\left(1+\beta_{w 1}\right)+\frac{1}{4} \varepsilon_{y}}-\gamma_{w 1}\right] \psi_{p e, w 1}^{i n c}, \\
& \psi_{p z, w 1}=\left[\frac{\left[\alpha+\gamma_{w 1}-(1-\alpha) \beta_{w 1}\right]\left(1+\beta_{w 1}\right)}{1+\gamma_{w 1}-(1-\alpha)\left(1+\beta_{w 1}\right)+\frac{1}{4} \varepsilon_{y}}-\beta_{w 1}\right] \psi_{p e, w 1}^{i n c} .
\end{aligned}
$$

Where $\alpha, \gamma$ and $\beta$ are defined by equation (175) and (172). $\gamma$ and $\beta$ are functions of the optical thickness. For wedge $1 \tau$ has the following values.

$$
\tau_{e z}=\frac{1}{4} \varepsilon_{y}, \quad \text { and } \quad \tau_{p z}=\frac{\varepsilon_{y}}{4(\alpha-1)} .
$$

A similar process may be completed for each wedge in the cell. The exiting fluxes for each wedge are presented below.

$$
\begin{gathered}
\psi_{e z, 2}=\left[\frac{\left(1+\gamma_{w 2}\right)^{2}}{\alpha\left(1+\gamma_{w 2}\right)+\frac{1}{4} \varepsilon_{y}}-\frac{\gamma_{w 2}}{\alpha}\right] \psi_{p e, w 2}^{i n c}+\left[\frac{(\alpha-1)\left(1+\gamma_{w 2}\right)^{2}}{\alpha\left(1+\gamma_{w 2}\right)+\frac{1}{4} \varepsilon_{y}}+\frac{\gamma_{w 2}(1-\alpha)}{\alpha}\right] \psi_{p z, w 2}^{i n c} \\
\psi_{p z, w 3}=\left[\frac{\alpha\left(1+\beta_{w 3}\right)^{2}}{(1+\alpha)\left(1+\beta_{w 3}\right)+\frac{1}{4} \varepsilon_{y}}-\frac{\alpha \beta_{w 3}}{\alpha+1}\right] \psi_{e z, w 3}^{i n c}+\left[\frac{\left(1+\beta_{w 3}\right)^{2}}{(1+\alpha)\left(1+\beta_{w 3}\right)+\frac{1}{4} \varepsilon_{y}}-\frac{\beta_{w 3}}{\alpha+1}\right] \psi_{p e, w 3}^{i n c} \\
\psi_{e z, w 4}=\left[\frac{\left(1+\alpha+\gamma_{w 4}\right)\left(1+\gamma_{w 4}\right)}{1+\gamma_{w 4}+\alpha+\frac{1}{4} \varepsilon_{y}}-\gamma_{w 4}\right] \psi_{p z, w 4}^{i n c}, \\
\psi_{p e, w 4}=\frac{1+\alpha+\gamma_{w 4}}{1+\gamma_{w 4}+\alpha+\frac{1}{4} \varepsilon_{y}} \psi_{p z, w 4}^{i n c}, \\
\psi_{p e, w 5}=\frac{1}{\alpha+\frac{1}{4} \varepsilon_{y}} \psi_{e z, w 5}^{i n c}+\frac{\alpha-1}{\alpha+\frac{1}{4} \varepsilon_{y}} \psi_{p z, w 5}^{i n c},
\end{gathered}
$$




$$
\begin{gathered}
\psi_{p e, w 6}=\frac{\alpha-(1-\alpha) \beta_{w 6}}{1+\frac{1}{4} \varepsilon_{y}-(1-\alpha)\left(1+\beta_{w 6}\right)} \psi_{e z, w 6}^{i n c}, \\
\psi_{p z, w 6}=\left[\frac{\left(\alpha-(1-\alpha) \beta_{w 6}\right)\left(1+\beta_{w 6}\right)}{1+\frac{1}{4} \varepsilon_{y}-(1-\alpha)\left(1+\beta_{w 6}\right)}-\beta_{w 6}\right] \psi_{e z, w 6}^{i n c}, \\
\psi_{p e, w 7}=\frac{1+\alpha+\alpha \gamma_{w 7}}{1+\alpha\left(1+\gamma_{w 7}\right)+\frac{1}{4} \varepsilon_{y}} \psi_{p z, w 7}^{i n c}, \\
\psi_{e z, w 7}=\left[\frac{\left(1+\alpha+\alpha \gamma_{w 7}\right)\left(1+\gamma_{w 7}\right)}{1+\alpha\left(1+\gamma_{w 7}\right)+\frac{1}{4} \varepsilon_{y}}-\gamma_{w 7}\right] \psi_{p z, w 7}^{i n c}, \\
\psi_{p z, w 8}=\left[\frac{\left(1+\beta_{w 8}\right)}{(1+\alpha)+\frac{1}{4} \varepsilon_{y}}-\frac{\beta_{w 8}}{1+\alpha}\right] \psi_{e z, w 8}^{i n c}+\left[\frac{\alpha\left(1+\beta_{w 8}\right)}{(1+\alpha)+\frac{1}{4} \varepsilon_{y}}-\frac{\beta_{w 8} \alpha}{1+\alpha}\right] \psi_{p e, w 8}^{i n c} .
\end{gathered}
$$

The associated optical thickness for each wedge as functions of $\alpha$ and $\mathcal{E}_{y}$ are presented next.

$$
\tau_{e z, w 2}=\frac{\varepsilon_{y}}{4 \alpha}, \tau_{p z, w 3}=\frac{\varepsilon_{y}}{4(1+\alpha)}, \tau_{e z, w 4}=\frac{1}{4} \varepsilon_{y}, \tau_{p z, w 6}=\frac{\varepsilon_{y}}{4(\alpha-1)}, \tau_{e z, w 7}=\frac{\varepsilon_{y}}{4 \alpha}, \tau_{p z, w 8}=\frac{\varepsilon_{y}}{4(1+\alpha)} .
$$

We can use these expressions to develop our $g$-factors. Because of the algebraic complexity of this exercise the specific definitions of the $g$-factors are not presented. This development was completed using a symbolic math program.

\section{4. Determination of the Truncation Error}

We can now substitute the $g$-factors into equation (184) to find an expression for the attenuation through an infinitely long row of cells. This result is not presented here because of its complexity. But when expanded in a Taylor series about $\varepsilon_{y}=0$ it has the following form.

$$
a_{\text {discrete }}=2-2 \varepsilon_{y}+\frac{1}{96} \frac{240 \alpha^{4}+68 \alpha^{3}-134 \alpha^{2}+3 \alpha+4}{\alpha^{2}(2 \alpha-1)(1+\alpha)}+O\left(\varepsilon_{y}^{3}\right) .
$$

The Taylor series expansion of the exact attenuation is

$$
a_{\text {exact }}=2 e^{-\varepsilon_{y}}=2-2 \varepsilon_{y}+\varepsilon_{y}^{2}+O\left(\varepsilon_{y}^{3}\right) .
$$

As we expect, 2D UCBL matches the first two terms of this Taylor series expansion. Thus, it is limited to first order accuracy. 


\section{VITA}

\section{KELLY GLEN THOMPSON}

Los Alamos National Laboratory, Computer and Computational Sciences Division

Group CCS-4, P.O. Box 1663, Mail Stop D409, Los Alamos, NM 87545

E-mail: kt@mindless.com

\section{EDUCATION}

Ph.D. in Nuclear Engineering, Texas A\&M University, December 1999

M.S. in Nuclear Engineering, Texas A\&M University, May, 1994

B.S. in Nuclear Engineering, Texas A\&M University, December 1991

\section{EXPERIENCE}

Technical Staff Member, Applied Theoretical and Computing Physics Division, Transport Methods Group, Los Alamos National Laboratory, Los Alamos, NM (10/99 - )

Graduate Research Assistant, Applied Theoretical and Computing Physics Division, Code Integration Group, Los Alamos National Laboratory, Los Alamos, NM (5/98 - 10/99)

Research Assistant, Department of Nuclear Engineering, Texas A\&M University, College Station, TX $(9 / 96-5 / 98)$

System Administrator, Department of Nuclear Engineering, Texas A\&M University, College Station, TX $(9 / 96-5 / 98)$

Graduate Research Assistant, Defense and Nuclear Technologies, Lawrence Livermore National Laboratory, Livermore, CA (6/96 - 9/96)

Research Assistant, Department of Nuclear Engineering, Texas A\&M University, College Station, TX $(9 / 92-6 / 96)$

\section{PUBLICATIONS}

K. Thompson and R. Hart, "Microchannel Plate Efficiencies for Medium Energy Helium and Carbon Recoils in Pulsed Beam Time-of-Flight Applications, " Nuclear Instruments and Methods in Physics Research, A 371, (1996) 563-565.

R. R. Hart, L. A. Foster, B. D. Lansrud, K. G. Thompson, and P. E. Tissot, "Rutherford Backscattering Measurements at Medium Energies Using a Pulsed Beam Time-of-Flight System," Transactions for the ANS 1999 Annual Meeting, June 6-10, 1999, Boston, MA.

K. Thompson and M.L. Adams, "A Spatial Discretization for Solving the Transport Equation on Unstructured Grids of Polyhedra," Proceedings for the International Conference on Mathematics and Computation, Reactor Physics and Environmental Analysis in Nuclear Applications, Sept. 27-30, 1999, Madrid, Spain. 
This report has been reproduced directly from the best available copy. It is available electronically on the Web (http://www.doe.gov/bridge).

Copies are available for sale to U.S. Department of Energy employees and contractors from-

Office of Scientific and Technical Information

P.O. Box 62

Oak Ridge, TN 37831

(423) 576-8401

Copies are available for sale to the public from-

National Technical Information Service

U.S. Department of Commerce

5285 Port Royal Road

Springfield, VA 22616

(800) 553-6847 


\section{Los Alamos \\ NAT IONAL LABORATORY \\ Los Alamos, New Mexico 87545}

